



\section{CAROLI A LINNE'}

EQVITIS AVR. DE STELLA POLARI S. R. M. SVEC. ARCIIIATRI

MED. ET BOTAN. PROF. IN ACADEM. VPSAL, REL.

M
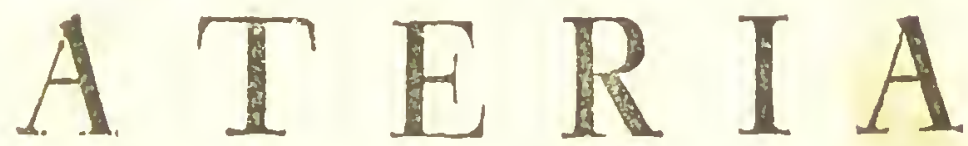

$\mathrm{M}$

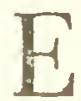

$\mathrm{D}$

I

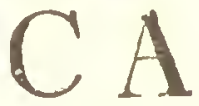

EDITIO QVINTA AVCTIOR

C V R A N T E

\section{IO. CHRIST. DAN. SCHREBERO}

SEREN. MARGGR. BRAND. ONOLD, ET CVLME. A CONSIL. AVL. MED. BOTAN. HIST. NAT. ET OECON. FROF. P. O. I N ACADEMIA FRID.

A LEX. E R I, A N G E N S I.

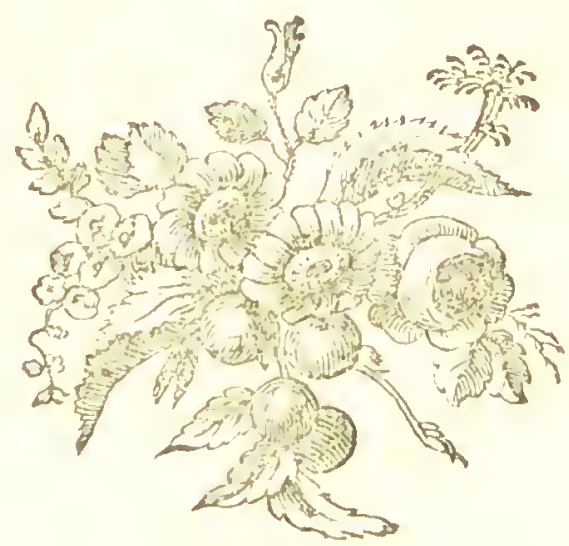

LIPSI $\triangle E$ ET ERLANGAE APVD WOLFGANGVM WALTHERVM Clo Io C C LXXXVII. 
Digitized by the Internet Archive in 2016 with funding from Wellcome Library

https://archive.org/details/b28776276 
IL.LVSTRI ET EXPERIENTISSIMO

\section{CAROLO PETRO THVNBER G}

ORD. REG. WASAEI EQVITI

MIED. ET B OTAN. PROF. REG. ORDIN. IN INCLYTA ACADEMIA VPSALIENSI

ACAD. IMPER, NAT. CVR. ET REG. SCIENT HOLMIENS. SOC. PATRIOT. HOLM. SCIF, T. VPSAL. BEROLIN. LVND. HARLEM. AMSTELODAM. NIDROS. SODALI ACAD. MONSP. ET SOC. AGRIC. PARIS. CORRISP.

MIVLTIS IISQVE MAXIMIS VT IN REM MEDICAM ET NATVRAIEEM PRAESERTIM HERBARIAM ITA ET IN ACCVRATIOREM SIMPLICIVM MEDICAMENTORVM COGNITIONEM

MERITIS IMMORTALI

HANC NOVAM

SVMMI PRAEDECESSORIS

MATERIAE MEDICAE

ED I T I O N EM

AD QVAM ORNANDAM ET PERFICIENDAMET IPSE MVLTA CONTVLIT

IN OBSERVANTIAE TESSERAM

D. D. D.

1O. CHRISTIANVS DANIEL SCHREBER 



\section{P R A E F A T I O}

EDITIONI PRIORI

\section{MATERIAE MEDICAE}

REGNI VEGETABILIS

PRA E MIS A.

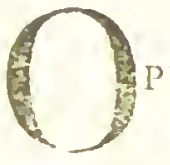

prscviva hoc de Materia medica in gratiam Difcipulorum, Studioforum Vpfaicnfium, comferipli. Curavi ut evaderet liber mole parvus, nec foret oneri difcentibus, heic etiam fufficit pauca, fed certa nolle, ut firmo doin fundamento valtifimam eruditionis molem fuperfruant tandem maturi Practici, inftitutis obfervationibus propriis felectilimis. Sciant autem eruditi Medici, me minime heic iptis feripfifle; tanca enim hodierna medicina fuperbit virrum medicinalium copia, ut non arcto libello capiatur, fed potius proprio pondere ruat.

SIMPLICIA tantum heic promo, ex quibus compofita dain conficienda et dijudicanda funt Quis enim de compofitis judicium ferret, qui ignarus effet fimplicium proprictatum? Sunt Simplicia defumta e triplici Nature Regno: e Lapideo, Vegetabili ct Animali; heic vigFTa Bur a tantum depromfi, qua ma* dimam confitume Materic Medic partem, alii tem. pori refervans cetera, cum debcant fingula Natura Regna propria methodo proponi*).

SVECICA OFFICINALA tantum in hOC opus intro. dusi, cum inltucrem Alumnos olim futuros per Svea.?

ciam

*) Prodierunt deinde fub differtationum academicarnm formal:

Alaberia medica eregno animuli 1750 recula in Alnmail. acad. Vol. 11 p 07.

Materiumedicalapidea.17;2. recufa in Amon. acad. Vol.111. p. 132. 


\section{PRAEFATIO.}

ciam Medicos, adeoque plantas vix alias, quam quas pharmacopoea holmensis (Medicinal-Orlningar. Stockb. 1742, oct.p.oc.) jubet, affumfi, et cas quidem omnes mulla exclufa, quamvis multa et cxclu. di poffent et deberent.

INVSITA TAs tamen nonnullas, viribus eximias et quibus commode carere nequeunt ingeniofs Medici, non potui non adderc, quarum tamen catalogum hcic fiftam, ne Pharmacopolas follicitent juvenes Me. dici de plantis, quas colligere et affervare, cx Conftitutionibus regiis, nondum tenentur; funt hx:

INDIGENAE. HORTENSES. EXOTICAE.

Rad. Britanuic.

Stip. Dulcamar.

Herb. Cotwla.

Bacc. Norlandice.

Sem. Datura.

Altrae

Barb. Hirci.

Balet. Cirvins.

Eupistorit.

Silep.

Telephii.

Lamii.

Livi cathars.

Linnare.

Myrt. Grabant.

Mufo. Cumatil.

Canisi.

Erefti.

Islandici.

Pedicutaris.

Pyxidati.

Vulvarias.

$\checkmark v \mathscr{C}$ ur $\boldsymbol{r}_{\text {. }}$

Xanthit.

Vitis idece.

Rad. Lapath. Jangu.

Herb. Humi veter.

Camphoratce.

Coridis.

Laurocerafi.

Meliffe.

Scroph. aquat.

Bacc. Bolladonce.

Fagopyri.
Rad. Alcantover.

Ginfeng.

Senegre.

Lig. Cafjines.

Ebrui.

Pavana.

Profuv.

Cort. Simsorstb.

Herb. Auvicular.

Acmella.

Fung. Melit.

Peragua.

Eruat. Fagara.

Nicul. Saver.

Pismentice.

Oryza.

Succ. Hypociflid.

Soja.

Sagu.

Vorsicis.

MET FODO in fingulis Plantis ufus fum conformi, caque compendiofifima, plantam unamquamque Octo $l i$ neis complcétcns, or dine eodem femper pofitis; filicet 


\section{PRAEFATIO.}

Nomess. $\mathrm{r}$.

Siynonymon. \&.

Locis. 3.

Pharmacesticums. 4.
Qualitas. 5.

Vis. G.

V fus. 7 .

Compofitum. s.

SPECIES reduxi ad recepta a me Genera et Claffes, ut certius innotefcerent Botanicis Plantæ. DIFFERENTIAM, qua Species a Congeneribus diftinguitur, fedulo adjeci, Floram svecicam de plantis 引ndigenis femper allegavi, ut Lectori Nomina vernacula, fimulque Loca indicaret, ne ab Exteris regio. nibus indigenas plantas arceffere cogantur Tharmacopœi Noltrates. HorTVM VPSaliensem fimiliter in fubfidium vocare nunquam intermifi de plantis in Hortis noftris nafcentibus, ut ex eo innotefceret Pharmacopois, quas plantas noftrum Solum facilius aut difficilius ferat, ne aut fruftra repetant multoties a nobis inftituta experimenta, aut ab exteris oris arceffant, quæ in noftris aque facile ac exterorum germinant Hortis.

Dvbras, in Specierum determinatione occupatus, miratus fum dari adhuc plurimas, in Officinis pharmaceuticis tritifimas et quandoque a reculo nati Salvatoris ufitatiffimas; he in tanta Botanices luce, quæ hodie eft, deftituuntur Defcripfionibus et Figuris aptis, ut circa has ideo cacutiamus fepe fereno die. Ne autem quis mihi objiciat, quod fpecies dubias feftimanter nimis determinaverim, en Catalogum carum, quas hafitans adpofui, ut fi cui Botanico in polterum aliquas ex hifce infpicere contin. gat in loco, ubi colliguntur pro officinis, de is follicite inquirat, qua fide nobis innotuerint.

Rad. lieisnrub. Cort. Cinell. alb. Suce. Ammon
Contriagerv. Asimir. 
Copisi.

Galariger.

Hermodat.

çaline.

Jpeininanh.

Ninfi.

Parpjuebrav,

Sichanminti.

Lign. Alop.

Afpalath.

Liralitime.

jernambise.

Ebennm.

Nephrit.

Rhodium.
Thymismat.

Herb. Syicts ind.

Sem. drif. Stellat.

Amomi.

Cocculi.

Cine.

Cubetix.

Dauci iret.

Mlyrob. indic.

lipllir.

Chelinl.

Paradiji.
Bdeilius.

Benzoï.

balf. Mecha. Peruy. Tolu.

Caramara.

Elemi.

Galbannm.

Myrrita.

Olibanmm.

Opopanax.

Sagaperum.

Styrax liqu.

Sang.Dracon.

Tacamahaca.

Rhus.

SYNONYMON unicum in fingulis plantis mihi pro compendio fatis crit, quod prixcipue a C. Baubini pimace mutuatus fum, unde reliqua defuni ct evolvi poffunt in plantis Luropais; at in Exoticis et Indicis felecifimum quoddam adduxi ab Horto Malabarico aut Pluckenetio vel álio dcfumtum, ut eo ccrtius innotcfceret planta: ubi rero Dubinm de planta fuit, plcrumque fignum (?) dubitationis adjeci.

NATALIA loco cujuscumque plantæ indicavi, ut contict unde fingula arceflantur, quæ notitia maxime Pharmacopolis neceflaria eft. Cum autem Plantæ Suecica indigcne etiam in adjaccntibus regionibus proveniant, ut evitarcm plura verba, Europam nofram feptentrionalem adpofui, quod plantam Suecicam indicat. Ad fincm Linex Tertix uno alterove vocabulo Culturam pharmacopois monftravi.

Vulgares dixi, que fponte apud nos crefcunt.

Cirres vero Exoticas, qua fomel in Hortos nofiros introducta, facile per fe fubfifunt es Annuo fenine rel Perenni radice. 
Manfuetas autem, qux more Animalium manfue. torum apud nos pulchre degunt, modoper hyememi tecta fubeant vel parum a geiu defendantur. Feras nominavi cas, que Ferarum inftar toto anno intra clauftra Caldarii coërcende et cuftodiendæ funt, ne aufugiant pereantre, qualcs vix Fru. Etum nofro fub climate ferunt.

Peregrinas dixi plantas, que Europæis Hortis vix unquam innotuere, adcoque e Pcregrinis terris arceffendie.

Durationem, ut Cultura co clarius innotcferet, unico verbo aljeci.

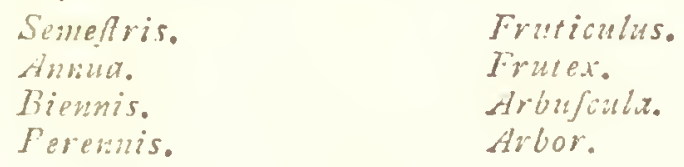

phariacoroeor vim Nomina, fiepius licet ab. furda, fancte fervavi, utpote complurium feculo. rum auctoritate, ratione legibusque exemta.

Simplicia, qure ex fingulis plantis defumuntur, fecundum modum nafccndi indicavi.

$\begin{array}{ll}\text { Radix. } & \text { Succus. } \\ \text { ligusus. } & \text { Flos. } \\ \text { Covere. } & \text { Frudus. } \\ \text { Folia. } & \text { Somina. }\end{array}$

Preparata partibus immifcui, ca tamen lege, ut præparatum femper fequatur partem ex qua praparatur, ut fi c. gr. olcumadfit, intelligatur iftud priparari ex parte proxime antegrena; funt preparata hac

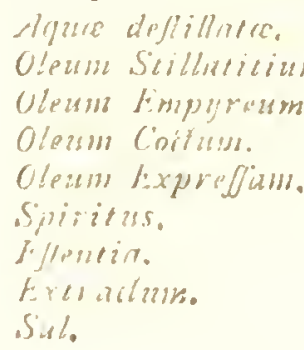
ronferva. sitripus. linh. cinulitum. Cosifualio. 


\section{PRAEFATIO.}

Qvalitates funt Senfibus externis cvidentia attributa. Odor a Naribus decerminatur et Volatilia indicat. Sapor a Lingua percipitur et aqua Solubilia manifeftat. Superficies Oculis plerumque dignofcitur, et Colores, Confiltentiam, aliaque determinat.

\begin{tabular}{|c|c|c|c|}
\hline APORE: & Fatuna, & In/ipidn, & Aquofa, Herbrica, \\
\hline & Oleracen, & Mucilaginofa, & Pinguin, Dulcin; \\
\hline & Aromatica, & Acria. & Pungentia; \\
\hline & $\begin{array}{l}\text { Calidu, } \\
\text { Salfa, }\end{array}$ & $\begin{array}{l}\text { Fervida, } \\
\text { Acida, }\end{array}$ & $\begin{array}{l}\text { Ignen, Urentia, } \\
\text { Anftern, Styptica; }\end{array}$ \\
\hline DORE： & $\begin{array}{l}\text { Amara, } \\
\text { Inodorn, }\end{array}$ & Fragrantin. & Suavcolentia. \\
\hline & Firtila, & Tetra. & Nanfiofa. \\
\hline PERF. & Coloratn: & vario colore, & Tinctorin. \\
\hline & $\begin{array}{l}\text { Offen, } \\
\text { linacia, }\end{array}$ & $\begin{array}{l}\text { Iriabilin, } \\
\text { Glutinofa. }\end{array}$ & $\begin{array}{l}\text { Porofa, Lactefo } \\
\text { Spumefientin. }\end{array}$ \\
\hline & Gummof $x$ & Refinof $a_{2}$ & Gummo \\
\hline
\end{tabular}

Fidem, quam fingulis fimplicibus adhibent Medici hodierni, interdurn uno alterove vocabulo indicavi. POTENTIAM agendi itidem in quinta Linea adpofui.
Dubin,
Infidn,
Eximin,
Heroica,
Praftantia,
Couta.

MODVM prefcribendi fæpius omifi, rarius addidi:
Externa,
Pretiofa,
Culinaria.

FREQVENTIAM præfcribendi hodie præfertim apud noftrates:

$\begin{array}{lll}\text { Rnirior, } & \text { Frequentia, } & \text { Trita, Ufitnta, } \\ \text { Infucta, } & \text { Infrequentia, Exoleta. }\end{array}$

VIREs in Sexta Linea habentur, qua theoreticam actionem medicamentorum docent. Sunt hac, fi paucas excipias, fepe mere fpeculaciva; hinc parcus in his fui. Ne termini hi artes numero oneri effent tyronibus, in proximis paginis methodice eos difpofui fub fequentibus claflibus: 


\section{PRAFATIO.}

$\begin{array}{ll}\text { Evacuantia, } & \text { Altcrantio, } \\ \text { Mufcularia, } & \text { Nerina, } \\ \text { Vifieralia, } & \text { Topica. }\end{array}$

vsvs in feptima Linea allegavi, fed parcus in his fui ; potuifem facile hic corraffie immanem copiam Medicamentorum, fed praftat hic pauca noffe quam multa; hujus enim oneri dudum fuccubuit Medıcina, nec formulis relticuenda, proinde in gratiam Tyronum non alios ufus produxi, quam quos multis experimentis probarunt Auctores, vel quos ipfe fufficienti experientia edoctus, fidos reperi; exanimavi enim, prefertim olim Nofocomiis claflis navalis prefećtus Medicus, Simplicia, quotquot licuit, omnia, et infida atro carbone, Tuta albo calculo notavi. $\mathrm{Ni}$ hilominus interdum coactus et anceps fignum Dubitationis (?) adpofui; at ubi maxime de medicamento fpecifico convictus, fignum Exclanationis (!) addidi.

composita pauca allegavi, cum mihi de compofitis plerisque fit peculiaris fententia; qui velit plura compofita eaque reccptifima fingulis plantis inferere, poterit hoc facile fupplere e Commelino vel alio. Collega meus, Clarifr. D. D. N. ROSEN, Archiater Regius, incepit laborantibus Compofitis medicas manus acidere, abfolvere non dum ipfi licuit obruto majoris momenti ncgotiis; optaveram rjus feleetas liormulas allegaffe, fi priss prodiflicnt, fed addant auditores poft hac, dum prodeunt, ut conjugrent conjungenda. HAEC abfolvi, Difcipuli dilcetifimi, unice ut vobis infervirem.

Daban VPSALIAE 1749. d. 28 . J゙annarii. 


\title{
LECTORI BENEVOLO
}

\author{
S. P. D. \\ E D I T O R.
}

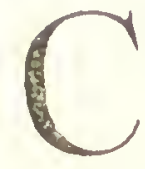

um matcria medica Liméi, viri inmortalis, libro aurco, officine librarice jam per pl.res annos caruifient, ncque ab excellenthimo auetore nova exfpetanda vidcatur editjo; 11 raltherus nolter, ubi a multis cum defiderari animaderterat, confilium cepit illius typis repetendi, atque ut editionis curam fufiperem iogarit. Difficilem fore intellexi, liquidem in illo aliqua crant immutanda, guedam illi altenda que ominia ad mencem auctoris efficere maxime videbatur arduum, Cedendum autem erat et Waltheri precibus ct auditorum defiderio; neque fpes certa decrat editionem qux novilfimas celeberimi auftoris de rebus unde medicamenta fumuntur contincret fententias, aliis quoque materix medicu cuitoribus et gratam fore ct utilem, quamvis poft. periluftr. a Linné non de fuerint, qui fimplicium medicamentorum notitiam quam aceuratifime tradiderint.

Impetrata igitur ab cxccllentiffimo auctore, præceptore limma obfervantia femper colendo, venia, preto illum denuo fubjiciendi; et de precipuis ad que in eo recenfendo attendendum eflet momentis. atmonitui, regno vegetabili, quod in prina cdicione fulum prodicrat, duo reliqua fecundum 


\section{PRAETATTO.}

novifimam fyftematis natur: editionem in alium ordinem redicta, adjunxi; illud vegetabilibus, in pharmacopolia nondum ubique receptis, fed a perilluftri auctore dignis, qua inter medicamcnta vulgo renalia referantir, habicis, auxi: nomina tam generum quam fiecicrum ex Sp. plantarum, Syfemate naturæ et Mantifis correxi, additis ubique harum nominibus trivialibus; loca natalia, ubi plura, vel certiora inveni, indicavi; qualitates, vires ufusque plures addidi, ea tamen adhibica cautione, ne quid propria auctoritate, quod a celcberrimo libri auEtore non profectum effe cenferetur, admifcerem.

Fontes, e quibus ifta haufi, funt perilluftr. a Linné opera, quæ polt editam Materiam medicam prodierunt; inprimis Syftema naturce, Genera et Species plantarum harumquc Mantifja utraque; Anconitates academice, et Clavis medicine; tum cjus in ma. teriam medicam prelectiones, a variis amicis omni diligentia calamo excepta. Scripfi Erlanged. XHI. April. clo 10 cel xxir.

In quarta hujus libri editione*) vocabula tech. nica pafim accuratius ad amuffim Clavis medicine b. auEtoris accomodavi; vocabula quitam, et paragraphum, qux amico prioris editionis curann mecum particnti, per feftinationem exciderant, refti-

*) Primum Mnteria medica regni vegefatilis Holmix 1749. animalis Vpfalia 1750. Inpidei ibid. 1792; tum Ira renga consjunitiun, curante Cel. Telari, V'enetiis 1, 62 colita funt. Tertia 1772. quarta 1781, utraque Erlangxe prodieruut. 


\section{PRAEFATIO.}

reftitui. Præcipue autem fimplicia quxdam, quibus b. auctor materiam medicam augcri voluit, in priorem editionem nondum recepta, ex ejus differtatione: Obfervationes in materiam medicam, Vpialix 1772. ventilata et in Ancenitatum academicarum tomo VIII. p.182. me curante recufa, et quibus. dam aliis, fuis locis inferui, et ad cjus mentem pauca quxdam mutavi. Addidi praterea in infimo quarumdam paginarum margine obfervationes auctoris in officinalia nonnulla, e fupra laudata differtatione, iisque aliquas immifcui adnotatiunculas, uncis [ ] ficut reliqua a menet ipfo paffim adfperfa, inclufas. Ne autem cum mole pretium libelii nimis augcretur, indices aliquos, minus neceffarios vifos, abrcidi. Tabulam xneam quoque, qua icon Opbiorrbize Mungos contineba. tur, cum non valde laudabilis eflet, omifi, me* liorem, cum charactere plantx emendato, pecu. liari libello editurus.

Quintam, quam philiatris nunc offero, editionem, ad normam Supplementi plantarum a. b. LiNNeo, Filio, Brunsvigx I 78 I. editi, paflim emendavi et auxi, et notas quasdam novas nonnullaque alia adfperfi, prxcipue autem diligenter curavi, ut ea prioribus quantum ficri polfet emendatior prodiret. Scr. Erlangx in Acad. Frid. Alexandr, d. I, Febr. clo 10 cclxxivir. 


\section{A VCTORES}

\section{PRIMARII ET VSITATISSIMI.}

I. D tos cor 1 Des Pedacius, Anazarbezs Siculns.

Libri IV. de Materia Medica.

Scripta Neroais atate. Proditi variis locis. Experta.

3. SCH R OE DER v Johannes. Medisus Francof.

Pharmacopeia medico chymica.

Vlim I64I. qu.

3. A v r Simon. Aledicus Regis Danix.

Quadripartitum Botanicum.

Argentorati 1667. quart. p. 660.

Compilata.

4. Ko

Regunm Vegerabile.

Bafilece 1688 quart. pars $1 ., 2.166$.

Bafilece 1696. quart. pars 2. p.27r.

Compilata,

5. R A Jvs Johannes. Theologus Cantabrigienfis.

Hiftoria Planrarum.

Londini 1686. fol. p. 1940.

Conquifita.

6. D A Lev s Samuel. Medicus Anglus.

Pharmacologia.

Lugd. bat. 1739. quart. p.459.

Botanic2.

7. Р о м т Petr.

Hiftoire générale des Drogues.

Parif 1604. fol.

8. VA LEN IIN I Mich. Bernh. Prof. Med. Gieff.

Mufeum Mufeorum.

Francof. 1704 Tom. I. 1714. tom. II. III. fol.

9. ToUr nefor T Jofeph. Pitt. Prof. Bot. Parif.

Hiftoire des Plantes des environs de Paris.

Parif. 1698 . ot. p. 545.

Chemica.

10. HER M A N v S Paulus. Prof. Bot. Leydenf.

Cynolura Materize Medicæ.

Argentor. 1726 quart. p.728. per J. BOECLER. Limata.

II. с н о м с Joh. Baptif, Medic Parijin. S. R. $P$.

Abrégé des Plantes ufuelles.

Parif 1715. dzod. Vol. 2.

Supplement.

Parif. Iт30. uod.

2. 2. R $\mathrm{k}$ Barthol Med. Doct.

Eotanologia Medica

Berutini I714. quart, Germanice, Compilatifima. 


\section{AVCTORES.}

13. g v С

Syecinen Medico-Practico-Botanicum.

Hufisia 2 т21. oillav.

14: no EC R R v s Johannes. Prof. Bot. et Chem. Argent.

Cynofura Materiae Medica 17) Continuatio.

Argent. I,jI. quart.

Limata.

5. BOER HA A I S Hermi. Prof. Bot. Lcydenfis.

Hifturial Plantarum.

Londini $I_{-3}$. oit.

Conquifita.

16. GE $S$ N $R$ V $s$ Joh. Albr. Archiater Wrirtemb.

Pharmacoprea lVirtembergica.

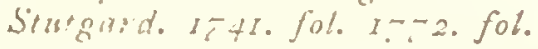

Nitida.

17. GEOFR O Siepl. Franc. Prof. Mat. Med. Par:if.

Trabatus de Materia Medica. Vol. 1. 2. 3.

Phrif. I, +ll. oit.

Elaborata.

18. $a b$ H A r. E R Albert Soc. R. Gotting. Profes.

Stippes Helvetix.

Goting. It72 fol. Bernce I-68. fol.

Selecta.

19. KvMn $\mathrm{x}$ s Ge Fiberh. Mercacor et Confli. Anboin.

Herbarium Amboinenle.

Aluflelod. I. $71-15.51$. fol. vol. 7 .

[20. с a r thevs r. Jo Friedr. Prof. Med. Francof.

Fundamenta Materia Medicr.

Fif. alt 1 iatr. 1:49. 1:07. tom. I. 11. ofi.

2I. LF. W I S IVill.

Experimental hift. of the mat. med.

Londini I GI. qu. anglice. Tiguri I J T. german.

22. GLEDIT S H Jo. Goitl. Prof. Bot. Berolin.

Verzeichnifs der Arzneygewichle.

berol. 5760 . oit

Wiffenfeh, der Arzneymittel.

Berol. oit. com. I. 2. $17: 9-15 S T$.

23 S P I

Inftitutiones Marerice Medicur.

Argent. oit. 1?:-

24. m v R R A y lol. Andr. Eques. Prof. Bot. Gotting.

Apparatus Medicamimum.

Gotting. oit. vol. 1. 1776 vol. 2. 1779. vo.. 3. 1783.

25. RER GIV S Pet. Jon. Trof. Jolm.

Mat. Med. e regno vegetabili.

Hoim. oit. tom. 1. 11. 1.5.8.1 


\section{A NONES.}

ร. Non poft rationem sedicina inventa fuit, fed poft inventam Medicinam RAT1O quafita eft. Celf.

2. Duo in Medicina Fvicra funt: Ratio et Expericntia; $E x$ perientin pracedit, Ratio fequitur; hinc Rationes in rebus medicis Experientia non condita, nilil yalent. Hoffin.

3. Mallem famofo EMPIRIco, quam fubtili Mechanico, falutem meam committere. Grelik.

4. BARBARI plus ad augmentum Medicaminum contulerunt, quam omnium atatim fchola. Brunn.

5. Serio fuadeo Medicina Candidatis, Evporista magno pere fibi commendata habeant. S. Paul.

6. Qui et multa et visia fcit, ille fapit. Contra Aefculap. 7. PAvcrs utatur Medicus Remediis, iisque Selectis. Trit.

8. selecta funt, quæ Medicum nobilitant.

9. Qui poteft mederi simplicibvs, dolofe et fruftra quxrit compolita. Villanov.

Io. Qui longas remediorum Formvlas prafcribit, aut dolo peccat aut ignorantia. Trit.

I. ( ) ui mifcet contraria, ægrum, pharmacopœum, et fe ipfum peffundat.

12. Plurima nedicamenta elaboravit chEMIA, fed nulla detexit.

13. Medicus notitia plantae deftitutus, de viribus ejusdem nunquam jufte judicavit.

14. Syftemate, Qualitate et Experientia eruitur omnis ufus plantarum.

15. Regnum vegrtaBLA praftantiffima, LAPIDEvM duriffima, ANIMALE paluciffina producit Medicamina.

I6. EsCVLevTa confervant, veNENA reftitunt fanitatem. 


\section{CANONES.}

1\%. Alimenta a Toxicis, uti Medicamenta a Venenis, non Natlira fed Dosis diftinguit.

18. Omne Nulva, quamvis optimum, Natura inimicum eft. 19. ADSVETA confervant, MVTATA frangunt Naturam. 20. CONTRARIA contrariis ct MORLI MORBIS curantur. 2r. Tituli renedi, Pyxides Venena habent. Agripp. 22. Medicanentis uti, nifi in velnementibus malis, svperVACANEVM Cil Colf.

23. Medicamenta Herorca, in manu imperiti, funt uti gladius in dextra furiofi. Trit.

24. Vbi vNica via falutis, en licet cum perieulo eft tentanda. 25. Satius eft $\Lambda$ NCEPS cxperiri remedium, quam nullum. 26. Quos Ratio nun curavit, firpe curavit Trmerstas. Ceif. 27. Jus vite fui ipfus MEDlco fapicnti tradat arger. 28. Natura arte adjuta, interdum facit MIRACVLA. 29. Felix eft ifte pulvis, qui venit rempore Crifeos. 30. Natvra repugnante, Medicina nihil proficit. Celf. 3I. PRVDENTIS eft MEDICI, cum, qui fervari nequit, non attingere, ne videatur occidific, quem ipfus fors interemit. Colf.

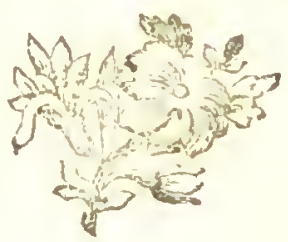




\section{P O N D E R A.}

Gr. Granum I. - - '- GvT'TA fepexqualis grano.

Э. ScRvprlus 20. - - - Grana 20. 5. DRACHMA 60. 3. - - Scrupuli 3. 5. VNCIA 480. 24. 8. - Dracbmee 8. tb. LIBRA 5:60. 280.96 . I2. I. Vncice I2. Gr. $\ni .3 .3$.

P. Prgillvs quod a digitis capitur. Dracbma $\mathbf{r}$. M. mantrulvs quod manu comp. Pugilli a f. $z$; F. Fascicvlus - - - Manipuli I2. Ch. Cochleabe - - Vucia . Q. MiEnsvrae Quadrans. - Libra r.

\section{S I G N A.}

\section{A NOBIS VSITATA.}

D. AQVA deftillata.

$\Omega$. SPIRITVS.

(D). olevm expreflum.

(O). OLEVM coEtum.

O. OLEvar ftillatitium.

O. OLEVI empyrcumaticum.

R. TINCTVRA.

宩. PVI.VIS. 


\section{T E R M I N I.}

Radices V. APFRIENTES majores.

Apii, Petrofelini, Foniculi, Rufci, Afparagi.

Radices V. APERIENTES minores.

Rubice, Graminis, Ononidis, Eryngii, Capparidis. Herbæ V. EMOLLIENTES.

Altbece, Mercurialis, Parietaric, Brance urfe, Betce.

Herbæ V. CAPILLARES.

Capill. veneris, Scolopendrii, Ceteracb, Tricbomanis, Rute murarie.

Flores IV. CORDIALES.

Borraginis, Buglofle, Role, Viole. Semina IV. CALIDA majora.

Carvi, Cymini, Anifi, Feniculi. Semina IV. CALIDA minora.

Ammios ver., Amomi ver., Apii, Dauci fylv. Semina IV. FRIGIDA majora.

Cucurbite, Citrulli, Melonis, Cucumeris. Semina IV. FRIGIDA minora.

Lactuce, Cichorei, Endivice, Portulace. 


\section{DOSES.}

Agarici - - 3j. Guttæ Gum. - Grxvj. Deioit. - 3jv. Sulpibur. Grvj. Eivrait. - - Эij. Hellebor. nigr, Rai. Эj. Alö̈s Subfantia - Oj. Extraitum - $\quad \exists \dot{p}$. Decoit. $\quad$ ziij. Extract.

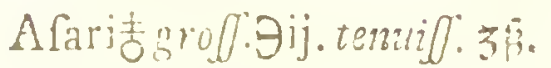
Fixtractum - $\quad \bar{\theta} \bar{\beta}$. Fol fublt. Nvi.inf.Nviij. BryonixRad.zij.Deroct.zj. Carchani Semina - $3 \mathrm{j}$. Cafic Pulpa - $\quad 3 \mathrm{j}$. Colocynthis Extract.Grjv. Croci Subfant. - Oj. Tinclur. - $3 \dot{3}$. Extrait. - $习 \dot{\text { r }}$ Cucum. afunin. Rad. $\theta \mathrm{j}$. Elaterii Extract. - $\theta$ j. Rejina. - Grij. Ebuli Cortex - 3 j. Semina - 3j. Efula Curtex - Grjv. Euphorbium - Grjv. Fumarix Succus - $\xi_{j}$. Gnidii Grana - Grjv. Gratiola Pulois - Oij. Succ. insp. $3 \bar{B}$. 


\section{DOSES.}

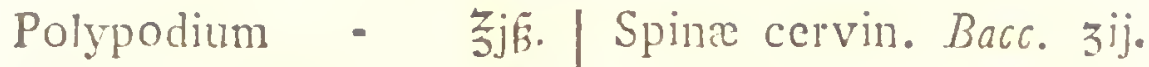

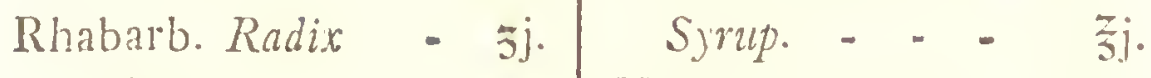

Effentia - 3i. Tabaci Extract. Griij.

Extract. - $\quad \mathrm{j}_{\mathrm{j}}$ Tamarind. pulp. - $\xi \bar{\beta}$.

Ricini Semina - Grjv. Decoet. - zij.

Rofarun folut. Jymup. 柿. Turbith - - 3 j.

Sagapenum - 3 F

Scammon. crud. Gr. vij.

Confect. Hamech. zjv. Elect. Lenitiv. - sj.

Diacrycl. $\quad \exists \overline{\text {. }}$.

Refina

Senna Subftant.

习习. Extr. Panchym. - $9 \overline{3}$. Decoct. - - 菃. Pulv. Cornach. -

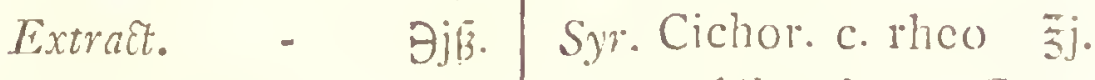
Soldanella fubl. - $\bar{j}$. Troch. Alhand. - Grjv. 


\section{SIMPLICIA.}

RADICES primo vere eraclicandie, antequam fo: lia germinant, confondenda in talcos, ficcandie iis umbra.

LIGNA colligenda tempore hyenali, findenda mox. STIPITES dicuntur rami arborum, qu colligendi tempore vernali, dum folia incipiunt crumpere. TVRIONES funt novelli caules, vel rami, qui nondum folia protulere, colligendi antequam indurefcant.

CORTICES tempore vernali defumendi, dum facillime a ligno fecedunt, aut hyeme.

HERBAE colligende quando incipiunt flöere, mox exficcande in umbra fufpenfe.

FOLIA legenda poftaram difforuit, fed antequam femina maturavit Planta.

FLORES colligendi ouamprimum dchifcere incipiunt, ante cafum pollinis antherarum.

Depurgati flores defumintur absque calyce \& piftillo, fola Corolla fervata.

ANTHERAE colligende antequam pollinem dimittant.

FRVCTVS legendi dum pericarpia fponte fefe aperirc incipiant.

SEMINA colligenda dum fponte a planta fecedant. Scmina valent quamdiu aquæ immifa fundum petunt; natantia vero rejicienda, paucillimis exceptis.

SVCCI fua fponte rel arte adjuti exfillant. GWMMI folvitur $\nabla$, non (D);

igne liquefcit aut llagrat.

RESINA folvitur (O), non $\nabla$;

igne ct liquefeit et fiagrat.

GVMMIRESINA folvitul +, partim $\nabla$,partim(Q). EXPRESsi SVCCl, lento igne infpifientur, antcquarn affervantur. 


\section{PRAEPARATA.}

AQVA. Planta (absque pravia fermentationc) cum Aqua fontilia deflillantur e vefica cuprea per alcmbicum ltanno obductum.

SPIRITVS. Planta uti Aqual deftillantur.

FERMENTATVS. Plantis contufis aflundatur parum aquæ calcutis, addito fermento; tent in vale patulo loco calido, ad juftam fermentationem, deftillentur.

VINOSI deftilentur ut prius, vino loco aqux, absque fermentatione.

SPIR:TV V'INI forti fiat deftillatio, uti vinof, qui ex plantis aromaticis fiunt.

OLEVM in ')fficinis quadruplex affervatu" :

STILLATITINA. Planta fubficca macerantur in aqua pel triduun, addito parum falis, deftillantur more aqua c vefica ad $\frac{\mathbf{T}}{3}$ repleta, olcum dein ful ernatans feparetur.

$V$ ú ex firitu fermentato, acjua debilitato, fumatur.

EMPYREVMATICVN. Planta refinofi defillantur per Returtam five per defeenfim.

CUCTVM. Ilerha macerantur in neo cxpreffo Colivarum), dein coquatur lente ad humidi confuntionem, esprimatur, culctur.

EXı'RESVM. Semina contura, calefacta in fartagine, agitata ne uftulcntul, cxcepta facculo linco, lubmifla proclo calido expriniautur.

SAL. CINERES. Planta recentes in fartagine, lamina ferrea tecta, lente conburantur ad perfectus cincres.

FIXVM. Cincres extriliantur aqua fontana, arpua decantetur, filtretur, evaporctur ald ficcitatem, parum calcinetur, folvatur aqua, coquatur ad pelliculam, cryftallifetur.

ESSENTIALE. Succus licrba recentis filtretur, in vitro lcute craporetur at $\frac{\mathrm{I}}{3}$, refidum reponatur in olla, tcctum oleo cripeffo, ftet per octo menfes, fal crytallifitum feparetur, cito aqua lirigida abluatur ct leniter exficcetur.

TINCTVRA AQVOOSA. Specicbus ficcis cum fale affundatur tepida, digerantur in luco tepido, extrahat aqua colorem, faporem; decantetur. 


\section{PRAEPARATA.}

SPIRITVOSA. Specicbus ficcis affundatur Spiritus vini ftent ill loco tepido, valic clalo, extrahat colorem, fapuren, odorem; decantctur, filtretur.

ESSENTIA eft Tinctura fpirituofa magis laturata et minus pellucida.

ELIXIRIVM, Efientia maxime faturata ex variis diverfis mistis, linc maxime opaca.

EXTTRAC'TM AQVOSTM., Tinctura aquofa plantr evaporetur ad confiftentiam.

SPIRITVOSVM. Tinctura fpirituofa evaporetur ad con. liftentiam.

AQVOSO SPIRITVOSVM. Plante ficce digerantur in Spritu vini, exprimatur, filtretur, forvetur Tincitura Spırıuofa. Kulidum aqua fontana digeratur, coquatur, filtretur, expreflum aquofum albumine clarificetur, fervetur Tinctura aquifa.

Mista Tinctura aquofa \& fpirituofa, lento igne evapo retur ad confiftentian.

SYRVPVS. Plantarum fucco aut infufo tox. adde Sacchari albi $\mathbb{W}$..j. $\mathbb{f}$. unica ebullitione Syrupus.

ROB. Baccarum fucci depurati Hojv. Sacchari $Z$ bj. coque in vafe figulino ad mellis confirtentian.

CONSERVA. Plantis recentibus incifis, adde Sacchari albi duplum, contufo probe in mortario marmoreo, lervetur in vitro.

CONDITVM. Radix f. Fructus carnofus, maccretur tribus dicbus, aqua quotidic renovatia, coque in aqua, cocta aqua lrigida abluatur, allunde Sacchartum ad confiftentiam Syrupi cuctum.

CONFECTJO. Scmina integra immittantur aheno confectorio fuper iguem collocato, allunde Sacchari clarificati cochlearia, moveatur fupra innem coutinuo.

Praparatio haece committitu plerumque sacchari confectoribus. 


\section{COMPOSITA.}

PVLVIS. Ilerbe fiec $x$ comminuta in fari- Pulver. nam cribratam.

ALCOHOL eft Pulvis fubtilifimus.

SIEF. P'ulvis pro oculis.

DENTIFRICIVM. Pulvis pro dentibus.

gVFFITVS. Pulvis groflus fpargendus Ríuderpulver. fupra prumas pro fumo.

SPECIES. Plantic ficca minution confcifix.

SACCVLVS. Specieset Pulveres fragrantes ficce, mixti, in Sacculo applicata.

2Iugenpuluer. Sntonpuloct.

Cucupbr. Sacculus pro capite.

EMPLASTRVM. Mafta in magdalcones cylindricos formata, confiftential in frigido folida, in calido liquefente, linteo inducenda, cui tenaciter adliarens, compolita ex Cera, Sebo, Kelinis, Oleis, lulveribus.

VNGVENTVM. Emplaftrum mellis confiftentia, pingue, calore liquelicens, non temaciter adliarens.

LINTMENTVM. Vinguentum olci confiftentia. Collyrimb Linimentum pro oculis.

CATAPLASMA. Puls cocta ex fpecicbus et Umidnlag. pulveribus in liquore,extcrne adplicanda.

BOLVS. Maffa confiftentia pultis denfio- biffen. ris ex I'ulveribus, Syrupo aut Rob.

ELECTVARIVM. Mafia confiftentia confe1- Inftmerge. va, ex fpeciebus f. Pulveribus, syrupis.

PILVLA. Mafli emplaftri confffentia, reda- Miffer. cta in globulos, ut integri deglutiantur.

SVPPOSITORINM. Mafta pilularis di- Stuljơpfein. giti figura, Reeto introducenda.

PESSVS. Suppofitorium pro vagina uteri. Snutrijąpfrin. TROCHISCVS. Pafta orbicularis fignata Errmfudilü. ex pulvere et mucilagine, in umbra ficcata. 


\section{COMPOSITA.}

MORSYLYS. Placenta rigida ex pulveribus Morfelle. ot faccharo al contitentiam tabulati cocto, cifuli lupra laminam.

ROTVLA. Norfulus fubrotundus, (Succo lepe impriegnatus), ellufis fupra laminam in orbiculos.

EXPRESSIO. Succus expreffus ex llantis Saft. tenelis fuccolis vernalibus, frigide propinandus.

EMIVLSIO. Lac paratum per contufionem ex Cotyledonibus cun aqua, modic: expreflione.

GVTTVIA. Liquores fpirituof, gutta- Eropfen. tim propinandi.

HAYSTVS. Potus cx varis aquis mixtis. Sunfurth. JTLEY'S. Haufus ex aquis, syrupo et Julep. acido.

MLTVRA. Haufus cx aquis, Pulveri- Miptur. bus \& Saccharato.

INFYSVM. Species aqux fesvida inditae, Shee. ut calida aqua absclue ebullitione proprictatem extrahat.

DECOCTVM. Species aqua aut vino co- Decoct. eta, ut liquor cbulliens cxtrahat proprictates.

GARGARISMA. Decolum iut haufus Burgelmaffer. pro collutione faucium.

EXI.MA. Decoctum tepide injiciendum cavitati mediante syringa.

Cly fura eft encma peranum injiciondum. Elyfrit.

EPITIEMA. Decostum corpore bibulo IImids!ag. applicandum calide.

BALNEVM. Decoctun calidum cum Iler- Ras. bis, procorporis humectatione ceterna.

SENICVPIVM. lialnem pro partibus pe- Sgrtobab. Ente inferioribus.

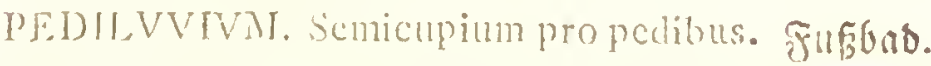
VAPOR. Halitus balnei ex olla per fellam Dumpfond. perforațan. 


\section{I R E S.}

Qualitates f. Principia Vegetabilium vim et actioncm fuamesferunt in corporis humani vel Liquida velSolida, vel ex Solidis Liquidisque compofita Vifcera. LIOVIDA vel cvacuando vel alterando. SOLID A vel in fibras Nerveas velMufculares agendo. SOLIDO. LIQVIDA velinternis vel Externis medendo.

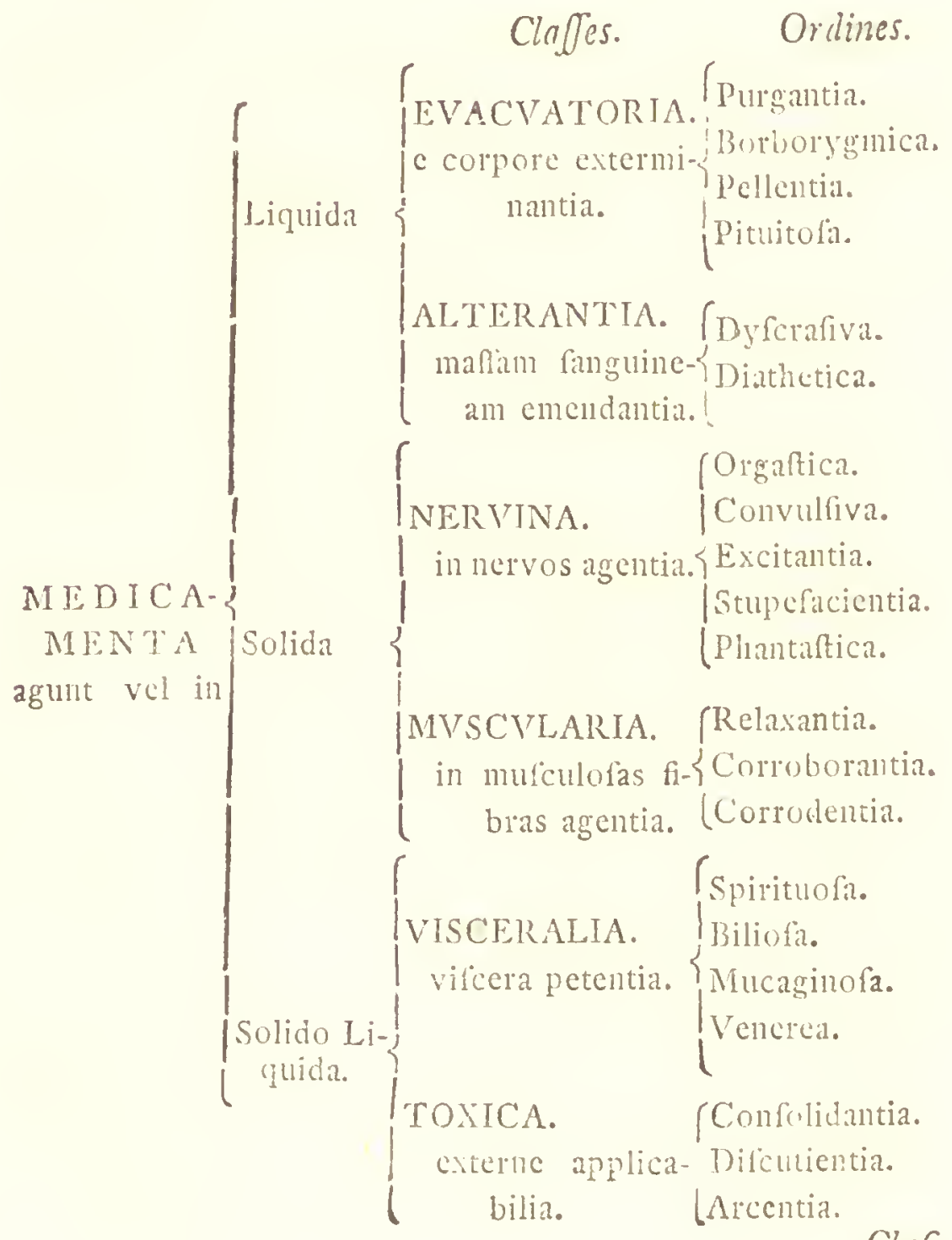




\section{Clapis $I$. \\ EVACVA N T A. \\ I. PVRGAN'IIA.}

cracuantia liquida intra tubum inteftinalem contenta. EMETICA. Fomitoria.

eracuant ventriculum per Oefopliagum. DRASTICA.

evalcuant inteftina \& ventriculum per anum violentifime. CATHARTICA. Purgantia.

evacuant inteltina per anum moderate.

- eCCOPROTICA. Lavativa, Laxantia.

evacuant inteftina per anum debiliffime.

\section{BORBORYGMICA.}

cvacuantia fatus intra tubum inteftinalem contentos. FLATVIENTA.

Flatibus abdomen diftendunt. RVCTATORIA.

Flatus per Oefophagum expellunt. CARMINATIVA.

Flatus per antm expellunt.

\section{PELLENTIA.}

evacuantia in Vafis contenta per truncum. DIAPIORE'ICA. Perfpirantin.

expellun per Tranfirationem. SVDORIFERA.

expellunt per Sudorem.

J)TRETICA.

expellunt Vinam.

E.MIENAGOÚA.

espellunt Menftrua.

ABOR'IIVA.

expellunt Fotum.

\section{PITVITOSA.}

evacuantia fecreta per caput. ERRIINA. Ptarmica.

Mucum e naribus cliciunt. 


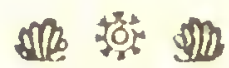

SIAIACiOGA. Apophlegmatifantia; Mafticatorin. Salivam ex lancibus \& ore eliciunt.

ENPICOORANTIA.

Screatim c Bronchis eliciunt.

\section{Choplis II. \\ A L T E R A N T I A. \\ I. J ISCR ASI A CA.}

alterantia humorum qualitatem acidam aut alcalinam. ANTTPILOGISTLA.

leutrefentiam aquofo diluunt.

REFRIGERANTHA.

Putredinem acidn cmendint.

BALSAMICA. Abforgentin.

putredini amaro refiftunt.

ANTACIJA.

Acido amaritic refiftunt.

ABSORBENTIA.

Acidum abforbendo infringunt.

\section{DI A'TE'TICA.}

altcrantia humorum Confiftentium \& mifcelam.

RESOLVENT'A.

Denfa attemuant.

INCIDENTIA. Atcmantia.

Vifcida attenlant.

MIVNDIFICANTIA. Diluentia, Aperientin.

Impura lavant.

EDVTCORANTIA.

Acria cdulcorant.

DEMVICENTIA. Tomperantia.

Acria frangunt.

OBTVNDENTIA.

Acria involvulut.

LVBRICANTIA. Mncilaginofa。

Acria invificant.

INSPISSANTIA.

Tenuia combinant. 


\section{Clafis III. \\ N E R V I N A. \\ I. ORGASTICA.}

angentia Funftiones vitales.

ALEXT'PERIA.

Ifalitum fragrantem reddunt.

STIMTLANTIA.

Secretiones incitant.

CALEFACIENTIA.

Calorem adaugent.

NVTRIENTIA. Alimontora.

Corpus reftaurant.

ANALEPTICA.

Vires infant.

\section{CONVVLSIVA.}

caulfantia nervorum convulfiones.

TVSSICVLOSA.

pulmones convellunt.

SINGVLTVOSA.

Cardian convellunt.

STERNTTATORIA.

Diaphragma convellunt.

\section{EXCITANTIA.}

mutantia Mentem.

EXIILARANTIA.

Animum addunt.

INEBRIANTIA. Fatuantia, Temulentantia, Pbantaftica.

Judicium confundunt.

\section{STVPEFA CIENT!IA.}

Suffocantia of cillationem.

PAREGORICA.

Orgafmum fedant. ANODYNA. Antipafmodica.

Dolorem mitigant. NARCOTICA.

Mentem ftupefaciunt. HYPNOTHC. Saporifera.

Soporem inducent. 


\section{Clafis IV. \\ M V S C V L A R I A. \\ I. RELAXANTIA.}

Laxantia tonum Fibrx mufculofic. HVMECTANTIA.

aquofo relaxant.

EMOLLIENTIA.

olcofo relaxant.

IMPINGVAN'TIA.

chylofo relaxant.

\section{CORROBORANTIA.}

Firmantia tomum Fibra mufculofie.

EXSICCAN'IIA.

Siccando corroborant.

TONICA. Corrobornutia.

amaro firmant.

ADSTRINGENTIA. Coriarin.

Styptico depfint.

SOPHISIICAN'TIA.

Genitalia adftringunt.

\section{CORRODENTIA.}

ABSTERGENTIA. Saponacea.

gluten fibrarum, abltergunt.

COSMETICA.

Superficicm fibrarum abradunt.

SEPTICA. Inflammatoria. Canfica.

Fibras in lammant.

VESICATORIA. Excorinntin. Exurentia. Exulcernutin:

Corroficin.

Fibras corrodunt. 


\section{Clafis $V$. \\ VISCERALI A. I. SPIRITVOSA.}

Nervos cxcitantia.

CEPHAtiCA. Nerina.

Caput confortant.

CARDIACA. Cordialin.

Cor corroborant.

\section{BILIOSA.}

Bilcm augentia.

IIEPATICA.

Hepar petunt, bilem gencrant. SPLENICA.

Splenem petunt? corroborant. STOMACIIICA.

Orexin excitant.

\section{MVCAGINOSA.}

Pulmones corroborantia lubricantiaque.

BECCHICA. Thornacica:

Muco acriufculo pulmoni dicata.

\section{VENEREA.}

Geniturx faventia.

APIIRODISIACA:

Venerem excitant.

VTERINA.

Fictuli favent.

IACTHERA. Cinactophora.

Lac adaugent. 


\section{Clafis VI.

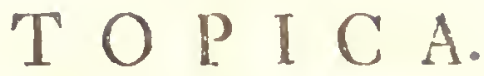 \\ I. CONSOLIDANTIA.}

Solutionem continui medicantia.

VILNERARIA. Cor?ulidantin.

Confolisant vulner:a.

GLVTINANIIA.

Conglutinant citius vulncra.

MITVRANTI八.

l'us moveint.

DIGERENTIA. Digefiza.

lis maturant.

SARCOTICA.

Camem, peresrina auferendo, generant?

CATHAERAETIC.

Ilyperdacolin aldertint.

CICATRISANIIA. Ipulotica?

Vlecra confolidata ficcant.

SISTENTIA. J funema.

Sanguinem filtunt.

\section{DISCVTIENTIA.}

IImores retundentia.

REIILLENTIA.

Materiem amandant.

LACTIFYGA.

Lac minument.

STERILITANIA.

Imprxgationcm prapcdiunt.

$$
\text { 3. } \triangle \mathrm{RCETIA}
$$

Animalculis noxia.

ANTHELMINTHTCA.

Vermes cnecant.

EXAN'TEMATICA. Be Ontion,

Acaros expellunt.

PHTIIIRIACA.

pediculus occidunt, 
C O N S P E C T V S

\section{MATERIAE MEDICAE.}

\section{REGNVM ANIMALE.}

Clafis $I$.

MAMMALIA.

$P R I M A T E S$.

Homo I Tapiens

BRVTA.

Eleplas 2 maximus

Trichechus 3 Manatus

FERAE.

$\begin{aligned} \text { Canis } & 4 \text { familiaris } \\ & 5 \text { Lupus } \\ & 6 \text { Vulpes }\end{aligned}$

Felis 7 Catus

Viverra 8 Zibetha

Vrfus 9 Arctos

10 Neles

GLIRES.

Lepus II timidus

Caftor 12 Fiber

Mus 13 Mufculits

PECORA.

Mofchus I 4 mofchiferus

Cervus is Alces

16 Elaphus

17 Tarandus

Cafra 18 Hircus

Ovis 19 Aries

Bos

20 Taurus
I

Fquis

Hippopot.

Sus

BELLVAE.

2 I Caballus

22 amphibius

23 Scrofa

CETE.

Monodon 24 Monoceros

38

12

Balana

25 Myfticetus

37

Phyfeter
14

13

15
26 macrocephalus 39

6
4
5
3
8
2
7

Clifis II.

A V E S.

$P I C A E$.

Corvus

27 Pica

23

AN'SERES.

Anas

28 Anler

29 Bofchas

24

25

GRALLAE.

Struthio 30 Camelus

GALLINAE.

16

17 Pavo 31 criffatus

27

18

I9

Phafianus 32 Galius

28

Tetrao 33 Perdix

29

20

21

PASSERES.

Columba 34 Oenas

30

22 llirundo 35 urbica 


\section{CONSPECTVS}

Clafis III.

'A MPHIBIA.

REPTILIA.

Rana 36 temporaria 37 Bufo

Lacerta 38 Scincus

SERPENTES.

Coluber 39 Vipera

40 Natrix

NANTES.

Acipenfer 4 I Sturio

\section{Clafis $I V$. \\ P I S C E S. \\ THORACICI.}

Perca

42 fluviatilis

ABDOMINALES.

Efox

43 Lincins

Cyprinus 44. Carpio

Clapis $V$.

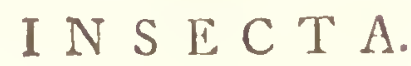

COLEOPTERA.

Lucanus

Meloë

HEMYPTERA.

Coccus

47 llicis

48 Cacti

LEPIDOPTERA.

Phalæua 49 Mori
HYIIENOPTERA.
Cynips

Apis

32 Formica

33

34

$\begin{array}{llll} & \text { Aranea } & 54 \text { domeftica } & 53 \\ 36 & \text { Scorpio } & \text { 55 europzeus } & 54 \\ 35 & \text { Cancer } & 56 \text { Pagurus } & 55 \\ & & 57 \text { Aftacus } & 56 \\ & \text { Onifcus } & 58 \text { Afellus } & 57\end{array}$

\section{Clafis. VI.}

\section{VER MES.}

INTESTINA.

41 Lumbricus 59 terreftris

Hirudo

60 medicinalis

58

42

43

Sepia 61 officinalis 60

Oftrea $\quad 6_{2}$ edulis $\quad 63$

Mytilus $\sigma_{3}$ margaritiferus $\sigma_{q}$

Helix 64 Pomatia 61

Dentalium 65 Entalis

\section{LITHOPHTTA.}

Madrepora 66 oculata

ZOOPHTTA.

46

Ifis

67 nobilis 66

Corallina 68 officinalis

48 Spongia

69 officinalis

V. 52 ? 


\section{MATERIAE MEDICAE:}

\section{REGNVM VEGETABILE.}

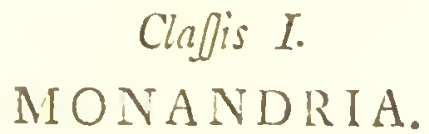

HOAOGYNTA.

Amomum 70 Zingiber

71 Cardomomum

72 Gr. paradifi 3

Coftus 73 arabicus

Maranta 74 Galanga

Curcuma 75 longa

Kæmpferia $7^{0}$ rotunda

Salicornia 77 herbacea

\section{Clafis II. \\ D I A N D R I A.}

MONOGYNIA.

Jafminum 78 officinale

Olea 79 curopra

Veronica 80 officinalis

\&) Beccabunga

Gratiola 82 officinalis

Verbena $8 ;$ officinalis

Nonarda $8+$ fifulofa

Rofmarinus 85 officinalis

Sdlvia

86 officinalis

87 Horminum

88 Sclarea

Collinfonia 89 canadenfis

TRIGTVIA.

Piper

9) nigrum

91 longum

92 caudatum

16

13

14

is

19

20
Claffis III.

\section{TR I A N D R I A:}

MONOG TNIA.

I Valeriana 93 officinalis

9f Phu

3 I

22

95 celtica

23

Tamarindus 96 indica

28

27

98 florentina

24

99 germanica

24

100 Preudo Acorus 25

Ior foetidiffima

Ic 2 tuberofa

26

Cyperus 103 longus

104 rotundus

DIGNNIA.

9 Saccharum 105 officinale 33

10 Phalaris 106 canarienfis 39

II Panicum 107 miliaceum 34

12 Avena 108 fativa 38

Is Hordeum 109 vulgare 35

17 Tricicum. 110 hybernum 36

III repens

32

TETRANDRIA.

MONOGRNIA.

Scabiofa II Succifa

Hedyotis

113 arvenfis
Afperula

IIs odorata 


\section{CONSPECTVS}

\begin{tabular}{|c|c|}
\hline Gali.ım & 116 verum \\
\hline Kubia & I 17 tindorum \\
\hline Penza & I 8 mucronata \\
\hline Platntago & $\begin{array}{l}\text { I19 major } \\
\text { I20 Plyllium }\end{array}$ \\
\hline Singuiforba & 121 officinalis \\
\hline Pingara & 122 Pteruta \\
\hline Trapa & I2; natans \\
\hline Lortenia & 124 Contrajerva \\
\hline Santaium & 125 album \\
\hline Camph. & 126 monfpeliaca \\
\hline Auchemilla & 127 vulgaris \\
\hline & $D I G Y N I A$ \\
\hline Curcuta & $\begin{array}{l}128 \text { europxa } \\
129 \text { Epithymum }\end{array}$ \\
\hline & ETRAG $T N I A$. \\
\hline Ilex & 130 Calline \\
\hline & Clapjis $V$. \\
\hline
\end{tabular}

\section{MONUGYAA.}

Lithofperm. 131 officiule 5?

Anchufa $\mathbf{3 2}$ officinalis 59

133 tinetoria 58

Cynogloff. I3t officinale 60

Pulmonaria 135 officinalis 62

Symphytum 36 officiuale $6 i$

Burago $\quad 57$ oflicinalis $\sigma_{3}$

Primula 138 veris officinal.64

Cyclamen 139 europium 65

Menyauthes 170 trifoliata 66

Lyfimachia Ifr Numularia 67

Alragailis 14: arventis

Sirigeita $x+3$ Anthetrin

Ophiorriza 144 Nungos

Plumbago 145 europiea

Convulumb liq6 Scmmonia
Convolvulus 1+7 Turpethum \&I 149 Jakipa 84 149 Mechoacanma 706 I50 Soldanella 83

Cincliona $15 \mathrm{I}$ officimalis $7 \mathrm{I}$ Prychotria 152 emetica 69 Coffea 153 arabica 70 Lonicera Ist Periclymenum63 155 Symphoricarp. 156 Diervilla

Coris $\quad 157$ monlpelienfis 80 Verbafcum is8 Thaplus go 159 nigrum 9t

Datura 160 Stramouium \$5 Hyofeyamus rer niger \$6 Nicotiana 162 Tabacum 87 Atropa 163 Mandragora 89 I6t íelladoma 89

Phyfalis 165 Alkelieugi 93 Solanum 166 Dulcamara 95 I6? nigrum $2 \%$

Capficum 168 : $113 n u$ um, 90 Strychuos 169 Nux vomica 77 170 colubrina 23 171 an:ara 172 Myxa 173 Cordia Rhamnus 173 catharticus 73 174 Frangula 73 375 Zizyplus 7?

Ceanothus 176 americanus Ribes x 7 ? rubrum 99 178 nigrum $\quad$ roo Hedera 179 Helix 98 Vitis 150 vinifera 97 Lagocia 18 a Cuminoides 2: Allamanda 182 cathartica 79 Vinca 183 minor ror 7) Nerium $18+$ antidyfenter:10= 32 Plumeria 185 alba 


\begin{tabular}{|c|c|c|}
\hline Afclepias & \multicolumn{2}{|c|}{ 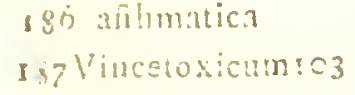 } \\
\hline IIerniaria & iss glaira & 104 \\
\hline \multirow[t]{5}{*}{ Chenopod. } & 189 Bonus henr. & 106 \\
\hline & Igo rubrum & IO? \\
\hline & sur Lutrys & 109 \\
\hline & 102 antheisuinth. & \\
\hline & I2; Vulvaria & $10:$ \\
\hline \multirow[t]{2}{*}{ Beta } & I9t vulgaris & 113 \\
\hline & I 25 Cicla & 113 \\
\hline Ulmus & 195 campentis & 105 \\
\hline \multirow[t]{3}{*}{ Geintiana } & 197 lutea & 110 \\
\hline & IgS Centaurium & 112 \\
\hline & 199 Amarella & III \\
\hline
\end{tabular}

Eryngium 2=0 fectidum SOI campefre

Sanicula 20: europia

114

Tuplenrum se3 rotundifolium 1,6 Tordylium 204 officinale $\quad 1+5$ Daticus 205 Carota 142 Conium 206 maculatum

Athamanta acr cretenfis $\mathbf{1 4 3}$ Pencedanumaso offinale $\mathbf{1 2} 6$ Ferula 207 Affa toetida is Laferpitium 210 latifolium $\quad 323$ 21 Siler 124

Heracleum 212 Sphondylium

Liguficum 213 Levificum 19 Angelica 2iq Archangelica $1=0$ 215 fyiveliris 121

Sinm 2:6 limbi 117

Sifun bubon

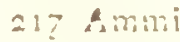
140 2:8 maredonic. I;;

21) Gibanum

Curioum 2:0 C;mimua

Ciculi 221 virola Actluta 222 Merm

Corizndruin 223 fativum
Scandix $\quad 2=+$ Cerefolium 137

Chicroph. 255 fyluefre 143

Inpelatoria 226 Ofruthim 122 Paininaca 227 fativa 130 228 Opopanax $13 \mathrm{r}$

Anethum 229 graveslens 147 $\simeq 30$ Foeniculum 146

Cartum 2il Carvi 138 vimpinslla $\approx 32$ Saxifraga 125 233 Anifum I4r Apium 234 Petrollinum 32 ? 235 giarcoles: 128 $T R ! G: N T A$.

Semecarpus 2,6 Anarardium 42 Rhus 237 Coriaria $\quad$ s5o 2 is Vernix I5 Cafine 239 Peragua 153 Sambucus 240 Ebulus I49 24 I nigra 148

Tamarix 242 gallica 154 TETRAG\}NTA.

Parnafia 243 paluftris 155 PENTAG $\eta^{\circ}$ !A.

Statice 244 l.monimm 159 Linum 245 ufitatifinam $1 ; 6$ 246 callititum 157 Drofera $\quad 2: 7$ undudilulia 158 Clepilis it. IEXARIRIA M!ONOG?:Y.

Promelia 2u: Mnabas

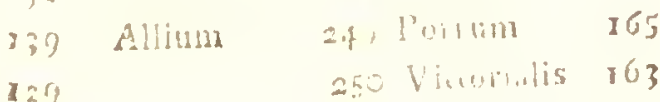
14a 2as filivim 164 1;5 25: Ceide $\quad 166$ 


\section{CONSPECT TS}

\begin{tabular}{|c|c|c|}
\hline Lilium & 253 candidum & I7 \\
\hline$\leq c i l$ & 254 marituma & \\
\hline Afpliodelus & sif rimofus & \\
\hline Affaingus & 256 officinalis & \\
\hline Convallaria & 257 nıajalis & \\
\hline & 258 Polygonatum & 10 \\
\hline Aloë & $25 y$ perfoliata & \\
\hline Ac:: us & 2 - Calamus & \\
\hline Achuas & 26: Sapota & \\
\hline erberis & 262 vulgaris & \\
\hline & $D / G N \Lambda ! A$ & \\
\hline Oryza & 263 fintiva & \\
\hline & $T R I G F N I A$ & \\
\hline umex & arsol fanguinens & \\
\hline & 265 acutus & \\
\hline & 266 aчpuaricus & \\
\hline & 26.7 fcutarus & \\
\hline & 268 alpinus & \\
\hline & 269 Acetufa & \\
\hline
\end{tabular}

Colchicum 270 antumuale

TETRAGTNIA.

Petiveria 271 alliacea

\section{Claffis VIII.}

\section{O C T A N D R I A.}

\section{MONOGMNIA.}

Tropxolum 272 majus

$18 \mathrm{t}$

Aniyuis

273 Elemifera

274 Opobalfam. 512

Lawonia 275 inermis

Vacciulum

$2 \% 6$ Mirtillus

277 Vitis idiax

182

184

185

Daphne

278 Mezercum

279 Laureola

\begin{tabular}{|c|c|c|}
\hline \multicolumn{3}{|c|}{ TRIGINIA. } \\
\hline \multirow[t]{4}{*}{ Polygonum } & 280 Biftorta & 189 \\
\hline & 28 Hydropiper & 187 \\
\hline & 28: aviculare & 186 \\
\hline & 283 Fagopyrum & 188 \\
\hline \multirow[t]{2}{*}{ Sapindus } & 284 Saponaria & 532 \\
\hline & $T R A G Y N I A$. & \\
\hline \multirow[t]{2}{*}{ Paris } & 285 quadrifolia & \\
\hline & Clnfis IX. & \\
\hline \multicolumn{3}{|c|}{ ENNEA NURIA. } \\
\hline \multicolumn{3}{|c|}{$M O N C G T N / A}$. \\
\hline \multirow[t]{7}{*}{ Laurus } & 286 Cinnamom. & 190 \\
\hline & 287 Caffia & $19 \mathbf{r}$ \\
\hline & 28* Campliora & 192 \\
\hline & 2xy Culilaban & \\
\hline & 2yo nobilis & 194 \\
\hline & 291 Sillafras & 193 \\
\hline & 292 Pecurim & \\
\hline
\end{tabular}

Anacardium 293 occidentale 206 TRIG $Y N / A$.

53 I Rlieum

2)4 Rhaponticum 198 295 palmatum

I97

\section{DECANDRIA.}

IIONOG\%NIA.

18 Sophora 296 heptaphylla

Auagyris 297 foctida

Hymenxa 298 Courbaril 515

Caflia 299 Fifula 199

300 Senua 200

jol alata.

Myroxylon 302 Peruifera $\$ 14$ Cofal- 


\section{MATERIAE MEDICAE.}

\begin{tabular}{|c|c|c|c|c|c|c|}
\hline Cexfalpina & 80 & brafilienfis & 205 & Portulaca & $3 ; 3$ oleracea & 260 \\
\hline & 304 & veficaria & 204 & Lythrum & 334 Salicaria & \\
\hline & 305 & Sappan & 203 & & $D I G I N I A$ & \\
\hline Guilandina & 306 & Moringa & 202 & $\Delta$ & 4 & \\
\hline Guajaten in & 307 & officinale & 207 & 301 & (I) & \\
\hline Dittamnus & 308 & albus & 208 & & $T R I$ & \\
\hline
\end{tabular}

$\begin{array}{lll}\text { Ruta } & 309 \text { graveolens } & 209 \\ \text { Tolnifera } & 310 \text { Balfamuin } & 201\end{array}$

Euphorbia 336 officinarum 254 337 Lathyris $\quad 256$ 338 paluatris $\quad 255$

llematox. 3i Campefcian.

Switeniz 3 i a Maluagoni

Quafia 313 amara

$31+$ Simarnba

Jednm 3.15 paluftre 910

DODECAGTNIA.

Semperv. 339 tedtorum $\mathbf{2 8 2}$

Rhododend. 316 Chryfanthum

Arbutus 317 Vua urfi $31 \mathbf{1}$

Pyrola 318 rotundifolia 212

319 umbellata

320 uniflora

Sityrax $\quad 321$ officinalis $\quad 227$

Copaifera 322 officinalis 513 $D / G N X / A$.

Saxifraga $3=3$ granulata 215

Gyplophila $32+$ Strulium

Saponaria 325 officinalis 214

Dianthus 326 Caryophyllus 213

PENTAGRNIA.

$\begin{array}{ccc}\text { Sedum } & 327 \text { Teleplium } & 217 \\ & 328 \text { acre } & 218 \\ \text { Oxalis } & 329 \text { Acetolella } & 216 \\ & D E C A G T N I A .\end{array}$

Phytolacca 330 decandra

\section{Clafjis XII. I COSANDRIA. MONOGTNIA.}
Myrtus $\quad 340$ communis $\quad 224$ 341 caryophyllataz26 342 Pimenta 225 Punica 343 Granatum 223 Anygdalus 34+ Perfica 230 345 communis 229 Prunus $\quad 346$ Laurocerafus 228 347 Cerafus 233 3.8 avium 233. 349 domefica 232 350 fpinola 23r DIG $I N I A$.

Cratægus 351 Aria

236

$T R I G K N I A$.

Clatjis $X I$.

DODEC $\triangle$ NDRIA.

MONOGrNIA.

Alarum 331 enropxum

Canella 232 alba

219

196

Sorbus

352 aucuparia

235

PENTAGINIA.

Merpilus 353 germanica 236 Pyrus 354 Malus 237

$\begin{array}{rr}355 \text { Cydonia } & 238 \\ & \end{array}$




\section{CONSPECTVS}

Spiraa

356 Filipendula

357 Vhmaria

POLYGNAA.

Rora

358 centifolia

$24 \mathrm{I}$

359 dambafcena

360 calsina

30. altia

liubus · 362 idaus

36 ; alcheus

364 Cirmmor. 247

Fragaria 365 vefia 245

Pocestulla 3:6i Auferiua

2.45

367 reptans

Tormentilla 368 erefta

Geum

369 urbanum

370 rivale

\section{Clafis XIII. \\ POLYANDRIA.}

MONOGWNA.

Capparis

371 fpinofa $\simeq 59$

Activa

372 racemora

247

$=48$

249

Chelidonium $37 ;$ majlis

Papaver

$37+$ Rhoeas

375 iomaiferum $=50$

Cambogia

376 Gutta

257

Nymphra

377 alva

2618

Bixa

378 Orleana

206

Tilia

379 europxin

Thea

3 so bohea

$20 \div 2$

264

Caryophyllus 381 aromaticus 265 Ciftus

382 creticus $26 \%$

DIGPNTA.

Pæonia
TRIGTVIA.

Delphinium 384 Confolida 268 385 Stuphifacria 269

Aconitum 386 Napellus 337 Anthora 270 TETRAGINA.

Cimicifuga $; \$ 8$ foctida

PENTAGNTA.

Aquilogia 389 vulgaris $\quad 272$

Nirgella 300 fativa 2 ?I

POLFGINA.

Wintera 391 aromatica

lllicium 392 anifatum 510

Anemone 393 Hepatica $=77$

394 Pulfatilla 276

395 nemorolia 275

Clematis 396 ręa

Ranunculus $; 97$ Ficaria 274

Helleborus 398 nige:

399 fretidus

Cinfis XIV.

\section{DIDYNAMIA.}

GIMNOSPERMIA.

Airga 400 pyramidalis 306

Teucrium for Chamxpitys 2\$? 402 creticum $\quad 286$

403 Marum 285

$40+$ Seurdium 289

405 Chanzdrys 288

Satureja 406 hortenlis $2 \mathrm{~S}_{4}$

407 capitata $\quad 283$ 


\section{MATERIAE MEDICAE.}

\begin{tabular}{|c|c|c|c|c|c|}
\hline Hylopus & 408 officinalis & $=20$ & \multicolumn{3}{|c|}{ Scrophularia 439 nodora } \\
\hline Nepeta & 409 Cataria & 291 & & 440 aquatica & \\
\hline Lavandula & 410 Spica & $\therefore 96$ & Limnea & $4+1$ borealis & \\
\hline & 411 Stachas & 297 & Sefamum & $44:$ orientale & \\
\hline \multirow[t]{4}{*}{ lentha } & 412 Auricularia & & Vitex & $4+3$ A tonus caltus & \\
\hline & $4: 3$ fyluelisis & 2.9 & \multirow[t]{2}{*}{ Acantisus } & $4+4$ mollis & \\
\hline & $41+$ crifpa & 278 & & & \\
\hline & $\begin{array}{l}415 \text { piperita } \\
416 \text { Pulerium }\end{array}$ & 280 & \multicolumn{3}{|c|}{ Clagis $X V$. } \\
\hline ecoma & 417 hederacea & 303 & \multirow{2}{*}{\multicolumn{3}{|c|}{ TETRADYNAMIA. }} \\
\hline mium & 418 album & 307 & & & \\
\hline etonica & 419 oflicinalis & $3 \circ \div$ & \multirow{2}{*}{\multicolumn{3}{|c|}{ SILICWLOSA. }} \\
\hline & $4: 0$ Tylvatica & & & & \\
\hline ium & 421 vulgare & 305 & \multirow{2}{*}{ Lepiditim } & $44 !$ & \multirow{2}{*}{322} \\
\hline urus & $4: 2$ Cardiaca & 308 & & 4h't lheris & \\
\hline \multirow[t]{4}{*}{ Origanum } & 4:3 Dictammus & 301 & Thlarpi & $4+1$ & \\
\hline & $43+$ cieticum & 300 & \multicolumn{3}{|c|}{$4 x^{2}$ Lurfa panor.324 } \\
\hline & 425 vulgare & 299 & \multirow[t]{3}{*}{ Cochlearia } & $4+9$ olfic & \\
\hline & 426 Majorana & $=98$ & & 450 Armoracia & \\
\hline \multirow{2}{*}{ Thymus } & 4:7 Se:pyilum & 282 & & & \\
\hline & 428 vulgaris & $88 I$ & \multicolumn{3}{|c|}{ SILIRVOSA. } \\
\hline \multirow[t]{2}{*}{ Melirfa } & 429 oficinalis & 294 & \multirow{3}{*}{$\begin{array}{l}\text { Cardamine } \\
\text { Sifymbrium }\end{array}$} & $45 \mathrm{I}$ pratenfis & \\
\hline & 430 Cal: & 295 & & 453 Nafurtium & 33 \\
\hline coceph. & $\begin{array}{l}431 \text { canaricise } \\
4 ; 2 \text { Moldavica }\end{array}$ & 292 & & $4 ; 3$ Sophia & 33 \\
\hline mum & 433 Baflicum & 302 & \multirow{2}{*}{ Eryfimum } & 454 oticinale & 33 \\
\hline \multirow[t]{2}{*}{ Pruneila } & 43 i vulgaris & 309 & & 455 Alliaria & 33 \\
\hline & & & \multicolumn{3}{|c|}{ Cheiranthus 456 Cheiri } \\
\hline \multicolumn{2}{|c|}{ ANGIOSPERMIA. } & & \multirow[t]{3}{*}{ Braflica } & 457 Napus & $3: 8$ \\
\hline irafia & officinalis & & & 458 & \\
\hline ularis & 436 paluftris & 314 & & 459 & \\
\hline rhinum & $n_{4.37 \text { Linatia }}$ & 313 & Sinapis & 4', nigra & $3: 6$ \\
\hline & $438 \mathrm{maj}$ & $31:$ & Raphanus & 462 fativus & 32 \\
\hline
\end{tabular}




\section{CONSPECTVS}

Clafis XVI.

MONADELPHIA.

$$
\text { DECAYDRIA. }
$$

Geranium 463 mofchatum 337 464 robertianum 336

POLTANDRIA.

Altixa 465 officinalis

Alcea 406 rofea

Malia 467 rolmudifolia

G. livpinn!'s herbacenm

Hovileus 469 Abehorchus

\section{Clapis XVII. \\ DIADELPHIA. \\ HEXINDTIA.}

Fumaria 4.0 belloofa

471 ollicinalis

OCTANDRIA.

Polygala $47 \approx$ Seriega

DECANDRIA.

Pterocarpus 473 Draco

$47+$ Santalinus

Genifta

475 canarienfis

4,6 tinctoria

Ononis

477 rpinofa

Lupinus

478 albus

Phateolus

479 vulgaris

Doliclios 480 Snja

Pifum

4 S fativum

Vicia

Eirum

482 Fabs

48; Lells

484 linvilia

Cicer
338 i40

339

$3+1$

$3+2$

Theobroma 493 Cacao 364

ICOSANDRIA.

Citrus

$49+$ Medica 366

495 Aurantium 367

POLTANDRIA.

344 Melaleuca 496 Leucadendra $34 ;$

Hypericum 497 perforatum 368 498 bacciferum

345

Clafis XIX.

$5: 2$ S Y NGENES I A. POLYGAMIA AERI ALIS.

347

345 Tragopogon 499 pratenle 372

$3 ; 7$ Scormonera $5 c 0$ humilis $37 \mathbf{r}$

3 io Laeluca 501 lativa 370

351902 virola

363 Leontodon 503 Taraxacum 368

j52 Hieracium $50+$ Pilofella 369

353 Cichorium 505 Intybus 373

$360 \quad 506$ Endivia 374

361 Aretiun 507 Lappa 375

399 Carduus 508 marianns 376 


\section{MATERIAE MEDICAE.}

$\begin{array}{lll}\text { Carlina } & 509 \text { acaulis } & 378 \\ \text { Carthamus } & 510 \text { tinetorius } & 377 \\ \text { Spilanthus } & 511 \text { Acmella } & 406 \\ \text { Eupatorium } & 512 \text { cannabinum } & 380\end{array}$

Santolina 513 Chamzecyp. 383

POLYGAMIA SVPERELVA.

Tanacetum 514 vulgare

515 Balfamita 382

Artemifia 516 Contra 51

517 Abrotanum 385

518 campeitris

519 portica

520 Abfinthium

521 valgaris

Gnaphalium 522 arenarium

$5 \geq 3$ dioicum

Tuffilago

524 Fiarlara

525 Petafites

Solidago $5: 6$ Virgasurea 393

Inula

527 Holenium 392

528 dyfenterica

Arnica

529 montana

395

Doronicum 530 pardalianch. 394

Bellis $\quad 531$ perenuis 425

Chrylanth. 5iz Lucanthem. 404

Matricaria 533 Parthenium fon

$53+$ Chan omilla 402

Anthemis 535 nobilis $40 \mathrm{r}$

536 Cotula $40 ;$

537 Pyrethrum 396

Achillea 538 Arreratum 399

539 Purmica 398

sto Millefolium 397

POIYGAMIA FRESTRANEA.

Centaurea 54: Cyanus

542 Behen

408

409
Centaurea 543 benediata

379

544 Calcitrapa.

POLYGANIA NECESSARIA.

Calendula 545 officinalis 407

MONOGAMIA.

Lobelia $\quad 546$ Syphilitica

Viola $\quad 547$ odorata 410

548 lpecacuanha 69

Clafis $X X$.

GINAIDRIA.

DIANDRIA.

Orchis 549 bifolia $4 \mathrm{rr}$

550 Morio $\quad 412$

Epidendrums5I Vanilla 418

$$
\text { IIE XANDRIA. }
$$

Ariftolo hia 552 trilobata

553 Serpentaria 416

$\$ 54$ rolunda 414 .

$5: 5$ longa 4 IA

556 Clematitis 415

OCTANURA.

Cytinus $\quad 55 z$ Hypociftis $\quad 220$

$$
\text { POLYANDRIA. }
$$

Arum

558 miaculatum

417

\section{MO NOE C I A.}

MON.NDREA

Cynomerium, 5, concinem sit Myrilica 560 mulchiala $5: 9$ 


\section{CONSPECTVS}

TRIANDRIA.

Phyhanthus 561 Emblica

\section{TETRANDRLA.}

$\begin{array}{lll}\text { Betula } & 562 \text { alba } & 42 \mathrm{r} \\ \text { Buxis } & 56 ; \text { fempervirens } & 423 \\ \text { Vrtica } & 564 \text { pilnlifera } & 4=0 \\ & 565 \text { divica } & 419 \\ \text { Morus } & 566 \text { nigra } & 422\end{array}$

PENTANDRIA.

Xanthium fG7 frumarium $4: 4$

POLYANDRIA.

Potcrium 568 Sanguiforba $4=5$

Quercus 569 Suber $\quad 4=7$

570 Robur $\quad 426$

Juglans $5 \%$ regia $4 ; 0$

Yagus 572 Caltanea $4: 9$

573 Pylvatica $\quad 428$

Corylus $57+$ Avellana 431

Liquidambar575 Ryraciflua 432

ADELPIIIA.

Pinus

$\begin{array}{ll}576 \text { fylveftis } & 434 \\ 577 \text { Pinea } & 435 \\ 578 \text { Larix } & 437 \\ 579 \text { Abies } & 436\end{array}$

Cupreffus 580 lemperviressa33

Croton 581 Carcarilia 470

582 tinctoriutn 448

583 'lictium 440

58 4 laccierum

Jatropha 585 Curcas 43

586 elaftica

Ricinas

587 communis

STNGENESIA.

Momordica 588 Elaterium 447

Cucubita 589 lagenaria 442 590 Citrullus $4+3$

Cucumis 59r Colocyuthis 446 592 Melo 447 593 fativus 445

Bryonia $59+$ alba 448

\section{Ciafis XXII.}

\section{I O E I A.}

\section{DIANDRIA.}

Salix

595 alba

449

TETRANDRIA.

Vifcum $\quad 596$ album

450

Myrica

597 Gale

45 I

$$
\text { PENTANDRIA. }
$$

Piftacia 598 vera

454

599 Terebinthus 452 600 Lentifcus 453

Spinacia 60 oleracea 456

Camabis 603 fativa $4 i 7$

Humulus 603 Lipulus 458

HEXANDRIA.

Smilax 604 Sarfaparilla 460 6o5 Clina

$46 \mathrm{I}$

OCTANDRIA.

39 Pop:lus 606 nigra 462 607 balfamifera 46 ; 438 Risodiola ocs rofea 472 $E N-$ 
MATERIAE MEDICAE:

ENIE.HNDTI.I.

Mercurialis 639 amma

DODECANDRIA.

Menifperm, 6ro Cocculus

IVONADELPIIIA.

Jumiperus Gi Sabina

612 communis

613 lycia

Ciframpelos 614 Pareira

SYNGENESTA.

Rulius 6 is aculeatus 468 616 Hypoglolf. 469

Clafis XXIII.

\section{P OIL Y G A M A.}

MONOECIA.

Veratrum 617 album

618 Sabadilia

Andropogon619 Schoenanthus $3 \mathrm{t}$ 620 Narủus 32

Parietaria 621 offinalis 472 Atriplex 622 hortenfis 473

Terminalia 623 Benzoin 195

Opluioxylon 624 ferpentinum 474

Mimola 625 nilotica 261

626 Senegal

627 Catechu

503

$D I O E C I A$.

Fraxinus 628 excelfior 475

629 Ormus $\quad 47^{6}$

Diofpyros 6;0 Ebenus so

Panax

6 is quinguefol.
TRIOECIA.

Ceratonid 132 Siliqua 455

Ficus 6;3 Carica 479

\section{Clafis XXIT. \\ CRYPTOGAMIA。}

\section{FILICES.}

Equifetum 6.4 arvente 479

Cycas $\quad 635$ circinalis $\quad 503$

Afplenium 635 Scolopendr. 497

677 Ceterach $\quad 483$

638 Irichomanes 433

6.39 Rutamuraria 488

Polypodium 6ac vuigare 485

641 Filix mas 486

Adiantum 642 Capill.vener.480

MIFSCI.

Lycopodium 643 clanatum 488

644 Selago 487

Polytrich. 645 commune 489

ALGAE.

Lichen 645 raxatilis 494

647 islandicus 493

648 pulmonarius 490

649 aphthofus 423

650 caninus $49 \pi$

651 cocciferus 496

652 Roccella

653 plicatus

495

FVNGI.

65+ Laricinus 497

655 Auricula 498 


\section{CONSPECTVS}

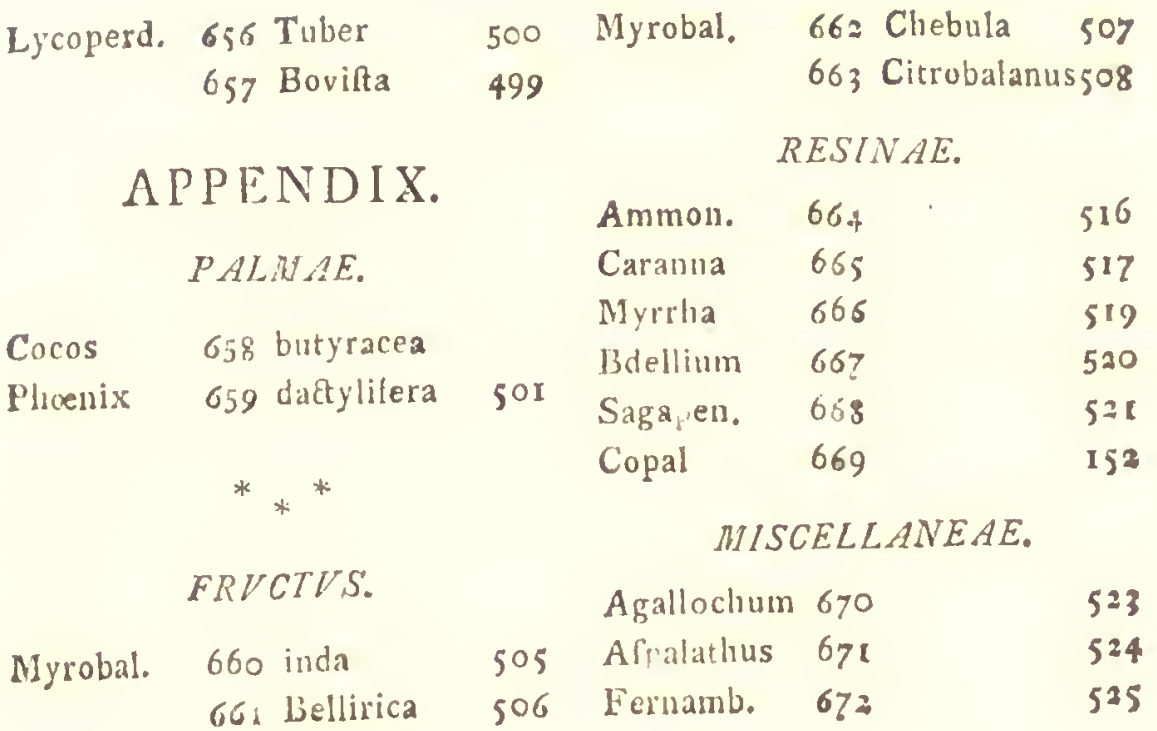

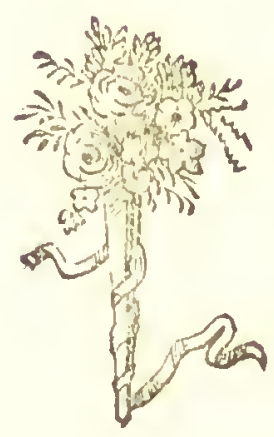




\section{MATERTAE MEDICAE.}

\section{REGNVM LAPIDEVM.}

\author{
Clajis $I$. \\ P E T R A E.
}

HVMOSAE.

Schiftus 673 Nigrica

$C A L C A R I A E$.

Narmor $\quad 67+$ rude

Gypfum $\quad 675$ vifuale

676 Alabafrum

Stirium $677 \mathrm{~g}$ gpfeum

ARGILLACEAE.

Talcum $\quad 678$ Rubrica

6.9 Smeetis

680 Nephriticus

Amiantus 68I plumofus

Mica

682 talcofa

59

9

5

10

8

ARENATAE

Silex

683 cretaceus
684 Carneolus I. $\beta$

Clafis II.

M I N ER A E.

$S A L I A$.

Nitrum

685 nativum

13

685 Cryftall. inont.14

6887 Fluor

$\alpha$ Hyacinthus

$\beta$ Amelhyous
Natrum

688 fontanum

689 glaciale

11

690 Tincal

69: Gemma nobilisi5

$\alpha$ Topuzins

$\beta$ Smaraglus $\zeta$

692 Granatis $\quad 48$

Muria

693 marina

16

694 fontana

695 montana

17

Ammoniac. 696 Sal

70

Alumen 69 ? commnne I8

628 romanum 19

699 Gemma pretiofa

$\alpha$ Rubinus $15 . \gamma$

E Sapphirus 15. $\varepsilon$

Vitriolum 700 martis $=0$

701 cyprium $2 \mathrm{r}$

702 album

22

$7 \circ 3$ plumbi

23

SILPHVRA.

Ambra 704 ambrofiaca 24. $\alpha$ 705 vulgatior $24 . \beta$

Succinum 706 eleetricum $\$ 5$

Bitumen 707 Petroleum $\quad 26$

708 Mumia

709 Afphaltum 27

710 nativus 29

711 Ferri 28

712 Auripigment. 30

Arfenicum 713 nudum

$3 x$

714 Sandaraca 3i
Borax 


\section{CONSPECTVS MATERIAE MEDICAE.}

\section{METALLA.}

$\begin{array}{rr}\text { Hydrargyr. } 715 \text { virgineum } & \mathbf{3 3} \\ 716 \text { Cimnabaris } & 34\end{array}$

Stibium 718 Atriatum 35.36

Zincum $\quad 789$ mineralifatum 38

720 Calaminaris 39

Vifmutum 721 nativum

Cobaltum 722 arfenicale $\quad 32$

Stannum 723 crynalliuum 47

Plumbum 724 nativim $\quad 46$

Ferrum 725 felectum 40

726 Smiris 42

727 Hamatites 41

728 Nlagnes 43

Cuprum 729 nativim 44

730 Lazuli $\quad 45$

731 Armenus

Argentum 732 nativum 49

Aurum 733 nativum 50

Clafjis III.

F O S S L L I A.

PETRIFICATA.

Helminth, 7 i4 Judaicus

735 Belemnites
Calculus

CONCRETA.

736 Bezoar

737 fellis

738 Margarita $\$ 6$

Tartarus 739 vini $\quad 54$

Aëtites 740 aquilinus 53

Pumex 741 Vulcani 52

Tophus 742 Ofteocolla $5 \mathrm{t}$

TERRAE.

Ochra 743 Ferri $6 \mathrm{r}$

$74+$ Aeris 62

Argilla 745 Lemuia. 698

746 tripolitana 60

747 Bolus

$\alpha$ alba $\quad \propto$

$\beta$ flava $\beta$

$y$ rubra $y$

$\delta$ viridis 65

748 Vmura $\quad 7$

$\beta$ Terra colon. 68

749 Creta 63

750 Gur 64

75 I damafcena 66

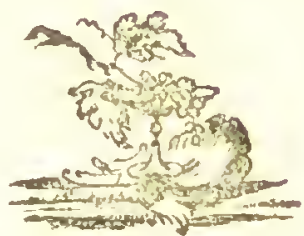




\section{R E G N V M}

A N I M L E. 



\section{$2-z^{2} x^{m}$ \\ CLASSIS I. \\ M A M M A I A. \\ PRIMATES. \\ HOMO.}

i. HOMO fapiens. Syjz. nat. i2. p. 28.

Locis: per totum terrarum orbeu, at

MVMIA in Aegypto.

PHARM. HOMINIS I. Cranium, rappatum, pree. paratum, $\Theta$. 2. Olla. 3. Axungia, Sal Sanguinis, Vrinx, $\Omega$.

cral. 1. 2. infipida, inodora, terreo - gelatinofa, 3. pinguis.

vis: 1. 2. abforbens. 3. emolliens.

vsve: I. Epilepfia?

comp. Pulo. de Gutteta, Arthetic:; Specific. ce. phal.; Mumiá

Superftitiofa, exoleta.

A 2

$B R V$

1. Circa AXVNGTAS obfervamus, quod liquidiores fint penetrantiores; quod recentes emollicuro, parum vero rancidae refolvendo agant. Onunes axungiae vim labent emolliendi, leniendi, praefertim vero Anatis, Anferis, Canis, Caponis, Caltoris, Galliuat, Hominis, Porci. Attenuandt et rofolvendi vis adfcribitur axumgiae Cati fylveftis, Lupi, Serpentum, Taxi, Viperarum. Vra, Vulpis. Calceaclendi, detergend et feptica vis adfignatur axmmgiae Lacii pifcis. 


\section{$B R V T A$. \\ E L E P H A S.}

2. ELEPHAS maximus. Syft. nat. 48.

Elephas. Raj. quadr. 123.

rocvs: India, Zeylona. Fera. PHARM. EBVR (Dens) x. crudum, raspatum, presparatum, 2. uftum. (Spodium).

QVal. I. infipida, inodora, terreo - gelatinofa. Vfitata.

Vis: 1. abforbens. 2. adftringens.

-vsve:

comp. Pulø. arthetic.; Haly abb.; Epil. marchnon.; pannon. rub.; Ipecific. cephal.; Confect. hyacinth. Smaragd.; Santalin.; Spee. Cord. temper.; de hyacinth.; diarrhod. alb.; diatr. Santal.

\section{TRICHECHVS.}

3. TRICHECHVS Manatus dentibus laniariis inclufis. Sy/t. nat. 4).

Manati f. vacca mariua. Raj. quadr. 393.

Locvs: Mare americanum, aliaticum.

Fera. pHarm. MANATI lapis (os petrofum).

QVAL. eboris.

vis: antifpasmodica?

Superfua.

rsvis: Calculus.

COMP. 


\section{$F E R A E$. $\mathrm{C} A \mathrm{NIS}$.}

4. CANIS familiaris cauda (finiftrorfum) recurvata. Sy/t. nat. 56 .

Canis. Gefner. quadr. gr.

LOCPS:

Cicur.

pharm. CANIS I. Axungia. 2. (Stercus) Album graecum.

CYAL. I. pinguis. Frequens, preftens. ris: I. emolliens, expectorans, 2. feptica, maturans.

rsis: 1. Tuflis, Phthifis. 2. Febr. intermittens, Colica? Dyfenteria?

comp. Vinguent, nervin.

5. CANIS Lupus cauda incurvata. Sy/t. nat.58. Lupus. Aldr. quadr:144.

Locis: Europa, maxime borealis. Minfietus. PHARM. LVPL I. Dens, 2. Hepar. 3. Axungia. QIAL. I. dura. 2 pinguis. Superfua. vis: 3 cmolliens.

rsvs: 1. Dentitio. 2. Hydrops? 3. Atrophia? COMP.

6. C ANIS Vulpes cauda recta: apice albo. Syft. nat. 59. Yulpes. Gefier. quadr. 55 .

torvs: Europa.

Manfietus. phir.r. VV L.PIS r. Pulmones praep. 2. Axungia. (NAL. 2. pinguis.

vis: 1. becchica? 2. emolliens.

vers: I. Hhthis? 2. Contractura.

comp. Lobos de pulmone vulpis. Vingue nervin. Oleum coct. vulpin. 


\section{FELIS.}

7. FELIS Catus cauda elongata fulco annulata, corpore falcis nigricantibus. Sy/t. nat. 62.

Felis vuigo Catus. Geliner. quadr. 98.

rocrs: Europa aufralior.

Cicur. PHARM. CATI SYLVESTRIS Axungia.

QVAL. pinguis.

vis: cmolliens.

Superfiua.

vsrs: Rheumatifmus, Variolae, Panaritium.

comp. Vnguent. nervin.

\section{VIVERRA.}

3. VIVERRA Zibetba cauda annulata, dorfo cinereo nigroque undatim friato. Sy/t. nat.65. Animal zibethicum. Hern. mex. 538. t. 580. [Buff. quadr. 9. p. 299. tab.31.]. xocvs: India utraque. PHARM: ZIBETHVM.

CVAL. Folliculus magnus inter anum et genitalia, pilofus: matcria febacea, oleofa, ambrofiaca.

Heroica, frequens. vis: diaphoretica, exanthematica, nervina, foporifera, aphrodifiaca, anodyna.

vsvs: Atecnia, Variolx, Morbilli, Scabies, Colica, comp. Pulv. dentifric. Balfam. apoplect.

\section{VRS V S.}

9. VRSTS Arctos cauda abrupta. Sy:2. nat.69. Vilis. Gefner. quadr. 14.

LoCVS: Enropa borealis.

Manfuetus. PHARM. VRSI r. Fel infpilfatim, 2 Axungia. QVAl. I. amara, 2. pinguis. Proficans, ufitata. 
VIs: . Alomachica, 2. colmetica, fophifticans, emolliens.

vers: i. Anorexia, Epilepfia.

comp. Vnguent. Martiat.

10. IRSVS Meles cauda concolore, corpore fupra cincreo fubtus nigro: falcia longitudinali per oculos auresque nigra. Sy/z. nat. $7^{\circ}$.

Taxus. Aldr, digit. 264 .

Locvs: Svecia et Europa varix regiones. Munfueta. PHAR:. 'TAXI Axungia.

QYAI.. pinguis.

Superflua.

$v$ is : cmolliens.

rests:

COMP.

\section{$G I R R E S$. LEPVS.}

II. LEPVS timidns cauda abbreviata, auriculis apice nigris. Sygt. nat. 77 .

Lepus. Gefner. quadr.69.

r.ocrs: Europa.

PHARM. LEPOR IS Tali.

Manfuetus.

Q $\lambda$ L. os primum metatarfi in fuffragine pofteriori. Superflua.

vis: abforbens, pellens?

vsus: Colica? Pleuritis? Epileplia? Dyftocia? cons. Pulv. pleuriticus.

\section{CASTOR.}

12. CAST OR Fiber cauda ovata plana. Sy/t. nat. 78 . Fiber 1. Caftor. Scbonev. icbtb. 33. [Buff: quadr. 8. p.282. t. 36.]

socvs: Lapponia, Sibiria, Canada. Manfuetus. PHAR.M. CASTOR VM I. optimum, fecratm, Pulvis, Extractum, Tinctura, 2. Axungia (Follicularis). A 4 QSAL. 
QVAL. I. Materia fotida, pinguis, in folliculo proprio juxta anum. 2. pinguis.

Heroica.

vis: I. carminativa, antifpasmodica, errhina, nervina, anodyna, emmenagoga. 2. emolliens.

rsvs: 1. Hyfteria! Colica! Vertigo, Epilepfia, Apoplexia, Lethargus.

eомr. Bal/am. vita; Eløet. Bacc. Laur.; Mithrid. Jhilon.; Androm.; Elix uterin.; Iimpl. hyfter.; Laul. opiat; Ol. coct. Callor.; Pil. de gum.; linguent. pot. rubr.

\section{V S.}

33. MVS Mufullus cauda elongata fubnuda, palmis tetradactylis, plantis pentadactylis: pollice mutico. Syft. nat 83 .

Mus domellicus vulgaris f. minor. Raj. quadr. 218. Locvs: ubique per Europam.

pharm. MVS I. combuftus 2. (Stercus) nigtum. QVAl. fotida.

vis:

2. purgans.

vsvs: I. Stillicidium? 2. Vermes,

COMP.

\section{PECORA. \\ $\mathrm{MO} \mathrm{S} \mathrm{C} \mathrm{H} \mathrm{V} \mathrm{S.}$}

14. MOSCHVS mofcbifern folliculo umbilicali. Syft. nat 91. [Pall. Jpicil. 13. pag. 3. tab. 4. Scbreb. mammal. $t$ 242. Buff fuppl.6. tab. 29.]

Animal mofchifurum. Raj. quadr. 127.

Locvs: China, Tataria.

Fera.

PHARM. MOSCHVS orientalis, Effentia.

QYAL: Folliculus fubumbilicalis, excretorius, fubfantia pingui unctuofa, fufca, fubacri, ambrofiaca. 
vs: nervina, cordialis, exanthematica, refocillans, aphrodiliaca.

rsis: Palpitatio, Debilitas, Cephalalgia, Colica, Hyfteria! Rabies!

cosip. Pulv. dentifric.; pro epithem. cord.; de guttet. Elix. vitae, Baifun apoplect.; Conf. alkerm. c. m.; hyacinth.; Eff. ambr. ver.; Spec. aromat. rolat.; cordial. temper.; de hyacinth.; imperator.; diamolch.; dulc. diarrh. abbat. Trocb. catech. c. m. de Gallia Moich. Ivily]. ad fornac.

\section{E R V V S.}

15. CERVVS Alces cornibus acaulibus palmatis, caruncula gutturali. Syjt. nat. 92. [Scbreb. maminal. tab. 246.]

Alces. Gefner. quadr.39.

Locrs: Suecia borealis, Ruflia, Canada. Manfuetus. ihara. ALCIS x, Cornu crudum, rappatum, preparatum. 2. ungula, preparata. CYAL. I. infipida, inodorata, terreo-gelatinofa. ris: 1. abforbens, demulcens. 2. abforbens?

visis:

2. Epileplia?

conp. Spec. cephal.; Pulv. epilept. marchion.; de Gutteta.

16. CERVVS Elapbus cormibus ramofis totis teretibus recurvatis. Syft. nat. 93 .

Cervus, Cerva, Himnulus. Gejner. quadr 79. Locvs: Furnpia.

Mimfiuetus. PHAR.r. CERVI I. Cornu tenellum, crudum, raspatum, 2. proparatum, 3. V/tum $\Theta, \Omega, \stackrel{0}{0}$, Gelatina, 4. Os de corde, 5. Priapus, 0. Sanguis, 7. Medulla, 8. Sevum.

QVAr. i. infipida, inodora, terreo-gelatinola. 2. pinguis, olcofa.

Jitata. 
13: r. abforbens, demulcens. 2. 3. 4. abforbens. 3. fibadfringens, anthelninthica?" $\Omega$ antilpasmodica, fudorifera, Cel. nutricns. 4. cordialis? antepileptica? alexipharmaca? 5.oblipans, aphrodifiaca? 6. cmolliens, lenitiva.

vsys: 1. 2. 3. Diamlna, Dyfenteria, 4. Verines? 5. Diarthod. 6. Dyienteria? Dyfuria, 7. Scorbutus. Excoriatura.

curen. Liou. C. C. fuccinat; Specif. cephal; Pulf: beroard. Senn. Cachect. dylenter. pueror. Conf. hyacinth. Spec cord. temperat de hyacinth. diamh. abbat. diamercurii Mynl. pro ptilan. alexiphamn. Trocb. de carab. Decoct. alb. Syd. Aqua C. C. citr. Waldeh. $\nabla$ de typhis.

17. CFRVVS Tarandus comibus ramofis recurvatis teretibus: fummitatibus palmatis. Sy/t. nat.93. [Scbreber. mammal. tab.248.]

Rangifer. Gelner. quadr. Izo.

Locis: Lapponia.

Cicur.

YHARM. RANGIFERI cornu rafpatum.

QYat. Cervi.

vis: Corvi, fed inferior.

Superfura.

vsrs: Cervi.

co.MP.

\section{A P R A.}

18. CAPR A Hircus comibus carinatis arcuatis, gula barbata. Syft. nat. 94.

Caper. Alitr. quadr, bifulc. 6rg.

rocrs: ardua Orientis.

Cicurr.

PHARM. HIRCL I. Sanguis. 2. Sevum.

HALDI 3. Sevum.

QVAI.

vis: 1. fudorifera, refolvens.

vsvs: I. Pleuritis, Contufura. 2. 3. Tulis.

Vjitata. 


\section{O VIS.}

19. OVIS Aizes cornibus compreflis lunatis. Syft.nat.97. Oris. Gejper auadr. 138.

Locrs: Africe ficca aprica.

PHARM. OVIS r. Siercis, 2. Oefypus.

Cicur.

Msi.. I. fotida.

IIS :

rsis: Icterus, Combuftura. 2. Luxatura, Contufura. comp.

\section{BOS.}

20. BOS Thums comibus teretibus extrorfum curva. tiv, palearibus laxis. Sy/t. nat. 98.

Bos. Gefieir. quadir. 55 .

Locrs: Boruffia, Yolonia.

Cictir.

pharm. BOVIS r. Medulla, 2. (Stercoris) Aqua For. ommizm.

TAVRL 3. Priapus, 4. Fel infpilfatum.

VITVI.1 5. Medulla.

(I.aitis) Butyrum, Cafeus, Serum.

RaA. I. 5. pinguis, oleola, 4. amara. Frequens. vis: r. 5. cmolliens, lenitiva. 2. refrigerans. 3. obftipans. 4. Aomachica.

vsvs: I. Scorbutus. 2. Rhevmatifmus, Febres. 3.

Diarrhxa, Dylenteria. 4. Timitus, Anorexia, Vermes.

comp. Vngu. de Arthanita, ad Vermes.

\section{BELLWAE. E Q V V S.}

2r. EQVVS Caballus cauda undique fetofa. Syft. nat. I00.

Equus, Caballus, Equa. Gefrer. quadr. 132. kocrs: Europa, parce Sponte, ubique. Cicur. 
pharm. EQVI Tefliculi.

QVAL.

vis : emmcilagoga?

Superfun, fuperfitiofa.

vsve: Colica? Menfes? Dyftocia? Pleuritis.

\section{HI P P POTAMVS.}

22. HIPPOPO'TAMVS ampbilizes pedibus quadrilobis. Sy/t. nat. Iог. [Buff.juppl.6.t.4.5.]

Locus: Nilus.

PHARM. HIPPOPOTAMI Dens.

QVAL. imfipida, inodora, terreo-gelatinofa. Superfun. VIs: abforbens, adfringens, mechanica, antifpasinodica?

rsvs: Profluvia Sanguinis, Epilepfa? Pleuritis? COMP.

\section{S V S.}

23. SVS Scrofa dorfo antice fetofo, cauda pilora Syjt. nat. 102 .

a. Aper. Gefner. quadr. 146. Aldr. bifulc. 1о13.

B. Sus. Gefiner. quadr. 872. Aldr. bifill. 937.

uocvs: $\alpha$ in Oelandia et Europa autrali; $\beta$ ubique.

Cicuir.

PHARM. $\alpha$ APRI Dens integer, preparatus, 2. Axungia. B POR CI 3. Stercus, 4. Axungia. eyal. I. infipida, inodora, terreo-gelatinola. 2.4 . pinguis. 3. fortida.

vis: I. abforbens, 2. emolliens.

vsvs: I. Pleuritis? 3. Profluvia fanguinis!

сомв. Pulv. pleuritic.

\section{CETE. \\ MONODON.}

24. MONODON Monoceros. Arted. gen. 78. Syn. II8. Sylt. nat:105. [Fabric. groenl. 18.]

Balae. 
Palina Narhwal. Siboner. icbtb. 28. [Klein. pifc. qiill. 2. tab. 2 fig. C.]

Locvs: Mare Atlanticum.

Fera.

PHARM. VNICORNV I. crudum, rafpatum, pre: paralquin.

Crat. r. infipida, inodora, terreo-gelatinofa. Rarior. VIS: I. abjorbens, demulcens.

vSFs: r. mechanicus.

comp. Spec. cephal. Pulv. epil. marchion.

\section{BALAENA.}

25. BALAENA Myfticetus naribus flexuofis in me: dio capite, dorfo impinni. Syft. nat. 105. [Fa-. bric. granl.20.]

Balina vulgo dicta. Gefner. aquat. iit.

Locvs: Mare Attanticum, Grœnlandia.

Fera.

Pharu. CETI Priapus.

RIAL.

Vis: aphrodifiaca? adftringens.

Superflua.

rsve: Prolluvia.

CO:IP.

\section{PHYSETER.}

26. PHYSETER macrocepborlus dorfo impinni, fiAula in cervice. Sy/t.nat. 170. [Fabric. granl.25.] Cete. Cluf. exot. izi.

Locrs: Nare Gronlandicum.

Fera.

PHARM. CETI Sperma.

evar. pinguis, ficca, fquamofa; alba, mollis, e ventriculis cerebri.

Preftans. vis: cmolliens, lenitiva, thoracica, refolvens, cosmetica.

vsus: Tuflis, Diarrhoa, Dyfenteria.

conp. Pulv. contra calum, Empl. de fpermat. ceti, Vingu. potab. rubr. 


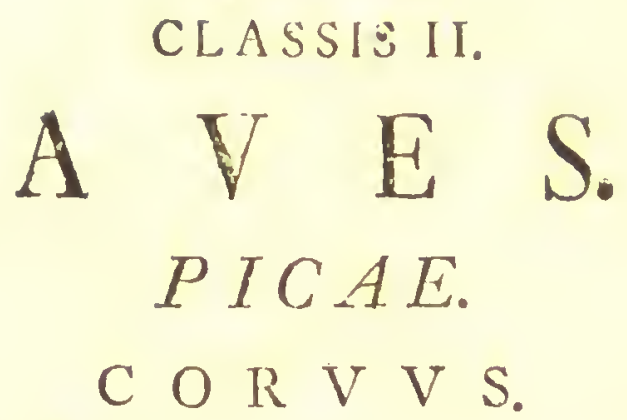

27. CORVVS Pica albo nigroque varius, cauds cunciformi. Syft. nat. 157 .

Pica caudata varia. Will ormitb. 87. t. 19. zocrs: Suecia ubique ad pagos. Manfuctus. PHARM. PICAE.

QVAL. Superfuua.

VIS.

VSVS.

comp. Aqua picarim compofita.

\section{ANSERES. \\ ANAS.}

28. ANAS Anfer roftro femicylindraceo, corpore fupra cinereo fubtus pallidiore, collo ftriato. Syft. nat. 197.

Anler domefticus. Gefner. av. I4I.

Locrs: Suecia borealis, nunc ubique

Cicur. PHARM. ANSERIS I. Stercus. 2. Axungia.

CVAL.

VIS: 2. emolliens.

2. pinguis.

Ifitata.

vsvs: i. Icterus.

cons. Vigur. refuntiv.

29. ANAS Bofcbas rechricibus intermediis (maris) recurratis, roftro recte. Syjt. nat. 205. 
Bofchas major. Alb. ornith. 2. p.89. t.100. Locvs: ubique in lacubus et ftagnis, hodic Cicar. PHARM. ANATIS ÄUngia.

Qval. Anferis.

Superfizc.

vis: Anferis.

vis:

comp. Vingu. pectorale, refuntiv,

\section{$G R \cdot A L L A E$, STR VTHIO.}

30. STRVTHIO Camelus pedibus didactylis. Syft. nat. 265 .

Struthio camelus. Will. ornith. 100.

Locrs: Arabix deferta.

Fera. PHARM. STRVTHIONIS ovorum tefl $x$.

Q AL. infipida, inodora, calcaria.

Superfura.

vis: abforbens.

vsvs: Calculus?

conp.

\section{GALLINAE. PAVO.}

31. PAVO criftatus capite crifta comprefla, calcaribus folitariis. Syft. nat. 267 .

Pavo. Gefner. av. 656.

r.ocis: Zeylona et India orientalis.

Cicur: pHAR.r. YAVONIS Stercus.

Qval. alba.

Preftans.

vis: nervina.

vsve: Vertigo! Epilepfia?

CU.Y. 


\section{PHASIANVS.}

32. PHASIANVS Gallus caruncula comprefla ver. ticis geminaque gula, auribus nudis, cauda compreila adfcendente. Sy/t. nat. 207.

Gallus gallinaceus et Gallina domeftica. Will. orn. Iog. $t, 26$.

Locvs: Pulicandor Indix.

PHARM: GALLINAE I. Ovorum Tëfx preparate; calcinate; 2. Viteli. (O). 3. Axungia. CAPONIS. 4. Axungia.

Cral. I. infipida, inodora, calcaria. 3. 4. pinguis. I. 2. prect mis, 3.4. juperfinu. vis: r. abforbens, calmat abforbens: adfringens, diuretica. 2. demulcens, nutriens, 3. 4 . emolliens.

vsrs: I. calcinat. Calculus, 2. Dyfentersa, Tuflis, Raucedo.

comp. Ingu. alb. ceruff. Martiat: pectoral. refumtiv. lithontripticum Dnx Stephens.

\section{TETRAO.}

33. TETRAO Perdix pedibus nudis calcaratis, macula nuda coccinea fub oculis, cauda ferruginea, pectore brunneo. Sy/t. nat. 276 .

Perdix cinerea. Fonft. av. 68. t.27. f. r. locvs: Europx noftre culta. Manfuetus. PHARM. PER DICIS Ylumx. QVAL. fumi fotida.

- Superflua.

VIS :

vsvs: fumi Epilepfia, Hyfteria.

COMP. 


\section{AVES. PASSERES. \\ $P A S S E R E S$. COLVMBA.}

34. COLVMBA Oenas carulefcens, cervice viridi nitente, dorfo poftico albo, fafcia alarum apiceque caudx nigricante. Syft. nat 279 .

Columba domeftica f. vulgaris. Will. orn. 130.

Locis: Sylva Europa, Afix, America feptentrionalis, ubique

Cicur.

PHARM. COLVMBAE Stercus.

QVAL.

vis: dubia.

Superfun.

rsvis:

conp.

\section{HIR V N D O.}

35. HIR VNDO urbica rectricibus immaculatis, dorfo nigro-cxruleicente, tota fubtus alba. Syjt. nat. 344 .

Hirmindo fylveftris, Gefizer. av. 564 .

locrs: ubique ad pagos per Europam. Manfueta. PHARM. HIR V NDO.

QVAI.

vis: dubia.

Superflua.

vivis:

roms. Aqua hirundium. 


\section{A M P HIB I A. REPTILIA. $\mathrm{R} \Lambda \mathrm{N}$ A.}

36. RANA temporaria dorfo planiufculo fubangulato.

Sy/t. nat. 257.

Rana itquatica f. innoxia. Gefiner. ovip. 46. [Rocfel. raii. p. I. t. I.]

rocvs: Europx paludes ubique. Manfueta. pHARM. RANARVM Sperma exficcatum, $\nabla$, (0). QVAL.

Vjitata.

vis: rec. refrigerans, repellens, $\nabla$ cofmetica, refrigerans.

vsrs: Sperma rec. Inllammatio, Eryfipelas, Combuflura.

comp. Empl. de Sperm. ranar. de ranis cum mercurio, Cataplafma.

37. R ANA Bufo corpore ventricofo verrucofo lurido fufonque. Syft. nat. 354 .

Bufo. Rond. aquat. 2. p. I2I. [Roef. ran. p. 85. t.20.] I.ocvs: Europa unbrula. Manflueti. pHARM. BVFONES exficcati.

QVAL.

Rarior.

vis :

vsvs: Panaritium, Hxmorrhagia? Congeftiones ad caput?

$\cos P$.

\section{A CERTA.}

38. LACERTA Scincus' cauda tereti mediocri apice comprefla, digitis muticis marginatis. Sy/t. nat. 365 . 
Stincus. Rond. pifo. 2. p. 23I. Seb. mus. 2. p. II2. t. $105 . f^{\circ} \cdot 3$.

r.ocrs: Acgrptus.

PHARM. STINCI MARINI.

Frra:

Rit.

vis: aphrodifiaca? diuretica?

Vfitata.

vsvs: Atecnia?

coup. Elect. diafatyr. Mithridat.

\section{SERPENTES. COLVBER.}

39. COLVBER Vipera feutis abdominalibus CXVTII,' fquamis fubcaudalibus XXII. Sy/t. nat. 375. Has. felq. it. $314 \cdot n .60$.

$V$ ipera. Gejner. Jerp. 124.

Locvs: Aegyptus.

Manfuetus. pharm. VIPERAE i. exficate, Pulvis, Trocbisci, $\Theta$, 2. offa. 3. Axungia.

QVAL.

Vjitata.

vis: r. alexipharmaca, diuretica, reftaurans, 2. ab. forbens.

vsvs: I. Syphilis, Lepra, Scabies ferina, Vlcus malignum, Venena? 3. Ophthalmia.

cons. Ther. Androm. Vngur. de tutia D. Sloane.

40. COLVPER Natrix futis abdominalibus CLXX, fquamis fubcaudalibus LX. Sy/t. nat.380.

Natrix torquata. Raj. quadr. 334.

Locvs: Europxe Rerquilinia.

Manfivetus. PHARM. SERPENTVM r. Spina dorfi, 2. Exuvix. 3. Axungia.

(3VAI.

3. Frequens. 
vis: 1. abforbens, 2. diuretica?

VSVS: I.

2. Dyftocia? Hydrops? Vulrus?

COMP.

\section{NANTES. \\ A C I P E N S ER.}

41. ACIPENSER Sturio cirris IV, fquamis dorfalibus XI. Syft. nat. 403. Mul. Ad. Fr. I. p. 54. tab. 18. f. 2 .

Sturio. Will. icbth. 239.

Locvs: Mare, fluvii maxini Europx. Manjuetus. PHARM. COLLA PISCIVM.

vis: glutinans, incraffans.

vsvs: Dyfenteria.

comp. Coctum gluten ex pinnis, inteftinis, corio ad pelliculam, malaxatum, refrigeratum, in pelliculas diductum.

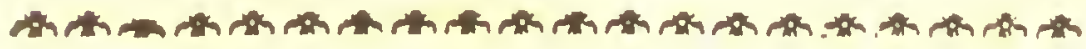

CLASSIS. IV.

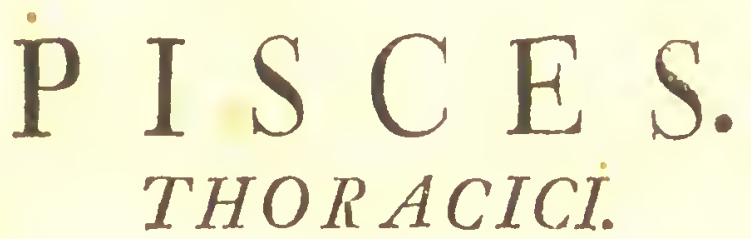

PERCA.

42. PERCA fluviatilis pimis dorfalibus diftinctis: fecunda radiis fedecim. Syft. nat. 48I.

Perca

41. [ Ichthyocolla optima vefica aërea Acip. Sturionis, et Acipenf. ftellati Pall., paratur; viliorem dat Acip. HwSo. tenaciorem Acip. ruthenus, et Silurus Glanis. Similem collau Cyprini Barbi aliorumque pircium velicae aëreae praebent, Pall. it. 1, p.s'g. 997 . Georgi it. s. p.677.] 
Perca fluviatilis. Salv. pifø. 226. f. 224 .

LOCrs: Lacus et fluvii ubique.

Mannfuetus. PHARM. PERCARVM Lapides preparati.

RIAL.

Vis: ahforbens.

Superfuta.

isus: Pleuritis? Colica?

comp. Specific. cephal. Pulv. pleuritic.

\section{ABDOMINALES. E S OX.}

43. ESOX Lucius roftro depreffo fubxquali, Sy/t. rat. 516 .

Lucius. Salv. pirc. 2. p. 188 .

Locvs: Lacus et fluvii ubique. Manfuetus. PHARM. L V C II I. Mandibulæ praparata, 2. Axungia. CVAL.

Vis: abforbens.

vsvs: 1. Pleuritis? 2. Vulnus.

comp. Pulv. dyfenter. pleurit.

\section{CYPRINVS.}

44. CYPRINVS Carpio pinna ani radiis IX, cirris IV, pinnæ dorfalis radio fecundo poftice ferrato. Sy/t. nat. 525 .

Cyprinus. Rond. pifc. 2. p.150.

LOCYS: Scanix pifcinae et Europa auftralior. Cicur. PHARM. CARPION IS Lapides.

QWAL.

vis: abforbens.

Superfut.

vsvi: IEpilepfia? Pleuritis? Colica? Calculus? comp. 


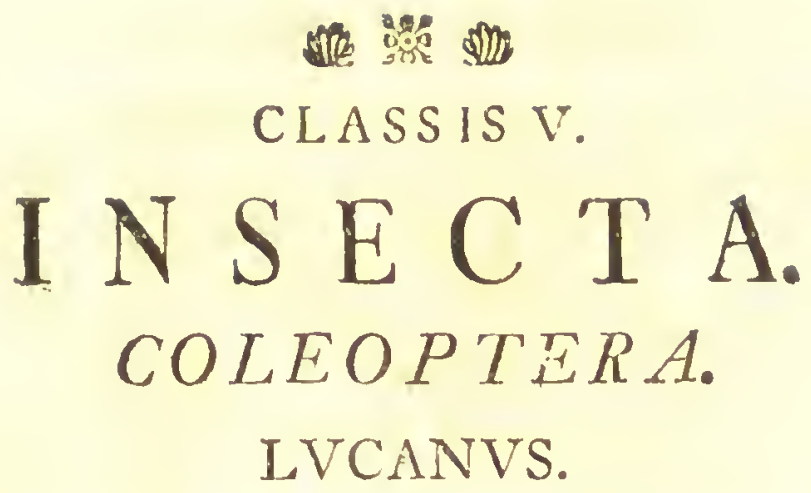

45. L VCANVS Cervus fcutellatus, maxillis exfertis apice bifurcatis latcre unidentatis. Sy/t. nat. 559 . Taurus volans. Olear. muf.27. t. 16. $f .5$.

Locvs: Europa querceta. Manfuetus. PHARM. SCARADAEI cornu.

QYAL.

vis: abforbens. Superfuna.

vsve: Dyllocia?

COMP.

\section{MELOE.}

46. MELOE vefeatorius alatus viridiffimus nitens, antennis nigris. Syjt. nat.679. Amoen. 6. p.135.* Sulz. inf. t. $7 \cdot f 55$.

Cantharis vulgaris officinarum. Raj. inf. 276 .

Locvs: Europr Liguftum, Syringa, Fraxinus, Lonicera. "Manfuetus. pharm. CANTHARIDVM Pulvis, Tinetura. CYAL. acerrima.

Heroica.

vis: inter. diuretica! aphrodifiaca, exter. corrofiva, exulcerans, excitans, revellens.

46. [Cantharides andiguorus crant Meloides navo - fafciatx, forfitan plures, prefertm Meloë calida Pall. irif. tab. E. fig. IX.

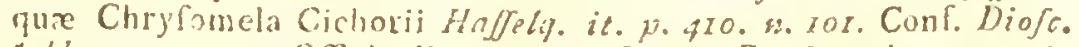
1.11. c.5\%. - Officinalis quoque Maloë Profonrabaems, mitior qquidem, tamen etiam veficans, adverfus rabiem cauinam lamidatus.] 
vsrs: inter. Ifchuria, Hydrophobia, Hydrops, Gonorrhai maligna. Exter. Febr. exanth. maligna, Pleuritis, Morbi Coporof, Delirium, Ophthalmia, Panalyis, Afhina, Vlcera cacoëthica. conp. Empl. velicatoriun.

\section{HEMIPTERA. COCCVS.}

47. COCCVS Ilicis Quercus cocciferx. Sy/t.nat.740. Kernes. Reaum inf. 4. t.5. [Ledermïll. micr.p. 72. t. 36.$]$

Locvs: QVER CVS coccifera. Sp. pl. 1413. Europre autralis. Fera. pHARM. CHERMES Grana, Succus infpilfatus, finctura.

Q 2 IL.

Preftans.

vis: fubalfringens, roborans, analeptica, aphrodiliaci.

rsis: molimina abortus.

conp. Conf. Alkermes, de Hyacinth. Smaragd. Iinctur. Pezoard. ex herbis, Spec, de hyacinth.

48. COC C V S Cacti Cacti coccinelliferi. Sy/t. nat. 742. Coccirella alis ideflituta, corpore rugofo. Brown. jam. 435 .

Scarabxus hemifphricus coccinellifer. Pet. gas. t. I. f. 5 .

Locus: Cactus coccinellifer in America. Fera. PHAR. COCCIONELLAL (Pupx). Q is. rubro-tinctoria. Praftans. vis: diuretica, leniter fimulans.

vises: Jydrops, Ifchuria, ficbres maligna. coss'. 'Tinclura facra Edinb. 


\section{LEPIDOPTERA. PHALAENA.}

49. PHALAENA Mori elinguis, alis reverfis pallidis: Anigis tribus obfoletis fufcis maculaque lunari. Sy/t.nat 817.

Bombyx. Rel. inf. 3. t 7. 8. Admiral. inf. t. 9. locvs: China, nunc in Europa pafim. Cicur. PHARM. SERICVM crudum, toftum. QYAL.

vis : antepileptica? adfringens?

Sufperfica. vsvs: Epilepfia? Hæmorrlagia? comp. Spec. cord. temperat, de hyacinth. dia. mofch. dulc.

\section{HYMENOPTERA. \\ CYNIPS.}

50. CYNIPS Rofe nigra, abdomine ferrugineo po. Alice nigro, pedibus ferrugineis. Syst. nat. 917. Reaum. inf. 3. $t$ 46. 47 .

Locvs: Suecix Rola fylveftris. PHARM. FVNGI ROSAR VM.

Manfueta. QVAL.

vis: foporifera? fyptica.

Superftitiofa.

vsrs: Hæmorrhagia.

comp.

51. CYNJPS Quercus folii nigra, thorace lineato, pedibus grifeis, femoribus fubtus nigris. Sy/t. nat. 918. RœS inf. 3.t.52.53.f. 10. Ir. Frifch inf. 2. t. $3 . f .5$.

Locvs: Turcix Quercus fempervirens. Manfueta. PHARM. GALLAE Turcicx.

51. [Optimas Gallas Q. Cerris [erre dicitur.] 
QVAL.

Fiequens, preftans. vis: Atyptica, adfringens, infpilfans, corroborans. rsvs: Hxmorrhagia.

COMP.

\section{A P I S.}

52. APIS mellifica pubefcens, thorace fubgrifeo, abdomine fufco, tibiis pofticis ciliatis: intus transverle ftriatis. Syft. nat. 955. Sulz. inf. t. 19. $f .123$. Apis. Mer. europ. 2. p. 19. t. I.

zocvs: Polonia, nunc ubique

Cicur. PHARM. MEL. I. commune, virgineum, defpumatum. $\Omega$.

CERA 2. citrina, alba, 0 . PROPOLIS 3 .

QVAL. I. dulcis.

1. 2. Eximia.

vis: I. edulcorans, abftergens, diuretica, maturans,

expectorans, 2. 3. digerens, emolliens, maturans. vis:

comp. Mel. Anthof.; Mercuriale, Rofar.; Vinlar.;

Oxym. Simpl. Scillit. Alliat. Cera rubra; viridis, nigra, figillatoria, arborea, ad barbam.

\section{FOR MIC A.}

53. FOR MIICA rufa thorace compreffo toto ferrugineo, capite abdomineque nigris. Syyt. nat. $9^{62}$. Formica inedia rubra. Raj. $\operatorname{inf.} 69$.

Scbaff. inf. t. 5. f. 3. elem. t.64.

Locvs: ubique in fylvis.

PHARII. FORMICARVM $\theta$, (), $\Omega$. eval. acida ellentialis, volatilis. Preffans. vis: excitans.

vsvs: Yaralyfis!

COMP. 


\section{$A P T E R A$. ARANEA.}

54. ARANEA domeftica abdomine ovato fufco: maculis nigris quinque fubcontiguis: anterioribus - majoribus. Sy/t. nat. 1031. Clerk. aran. 76.t.2. f.9. Araneus fublavus hirfutus, pratongis pedibus, domefticus. Lifi. aran. 59. n. 17.

zorvs: in feneftris ubique. pHARn. AR $A$ NEAE 'Tela.

QILA.

Manfuetn.

VIS:

vsvs: Vulnus.

comp.

\section{SCORPIO.}

55. SCORPIO curopeus pectinibus 18-dentatis, manibus angulatis. Sy/t. nat. 1038. Sulz. inf. t.23. f. 550 .

Scorpio. Raj. inf. 9. Raf. inf.3. p. 66.f. I. 2. socvs: Muri Italia, Africx. Fera. THARM. SCORPIONES AFRICANI exfeccati, (O). (ISAL.

Rarior.

VIS:

vsrs: Punctura.

cosp. Oleum inf. magnum.

\section{CANCER.}

56. CANCER Pagurus brachyurus, thorace utrinque obtule novem - plicatu, manibus apice atris. Syjt. nat. 1044 .

Cancer marinus. Fonfton. exfang. t. 5. f. 2. locrs: Oceanus. Manfuctus. pharm. CANCRORVM chela, preparatae.

QI.AL.

56. Ahforbentia e regno animali defumta haud inepte ad duas reducl poffun claffes: ad priorem pertinent illa, quate quidem 
QVAL. infipida, inodora, calcaria.

vis : ablorbens.

vsvs: Hypochondriafis.

comp. Pulv. bezoard. anglican.

57. CANCER Aftacus macrourus, thorace lavi, roftro lateribus dentato: bafi utrinque dente unico. Sy/t. nat. I051.

Aftacus Huviatilis. Rond. pifc.2. p.210. Ref. inf. 3. t. 54.55 .

Locvs: Lacus et fluvii Europx.

Manfretus. pharm. CANCROR VM oculi (Lapides), Solutio. QVAL. infipida, inodora, calcaria. vis: ablorbens.

vsve: Soda, Hypochondriafis.

comp. Pulv. pleuritic. ftomachal.

\section{ONISCVS.}

58. ONISCVS Afellus ovalis, cauda obtufa: Atylis implicibus. Syft. nat. 106r. Sulz. inf. t.24.f. I54. Afellus afininus $\mathrm{f}$. vulgaris. Raj. im $\int$. 4I.

Locvs: muri antiqui et parietes.

Manfuetus. PIARM. MILLEPEDES proparati.

QVAL.

vis. diuretica, incidens.

Vfitata.

vsrs: letcrus, Afthma, Dyfuria, Nephritis. comp. Pil. Scillit. Edinb.

CLAS -

abforbent, fed fimul vi porionis gelatinofæe demulcent. Numerantur huc animalium Offa, Cormua, Dentes. Ad po. fteriorem refermour gelatima demulcente denituta, ut Teftr. ovorum, Cancrornm chelae ot nculi, Telta? cochlex, Dentalium, Conclie etc. quo ctian fipectant animantium pitrtes jain dicte, dum varia arte et prie paratione gelatinofa parte funt orbate. Parum jivant abrorbentia haec, nifi vel aridn quodam regni vegetabilis prius lint impragnata, vel offendant acidusn in primiis viis; tun suim unu folum illud ahforbent deftountrue, fed eliam icmperatuem, blande refol. venten, diapnoicim et diactions exferere polfunt virtutem. 
CLASSIS VI.

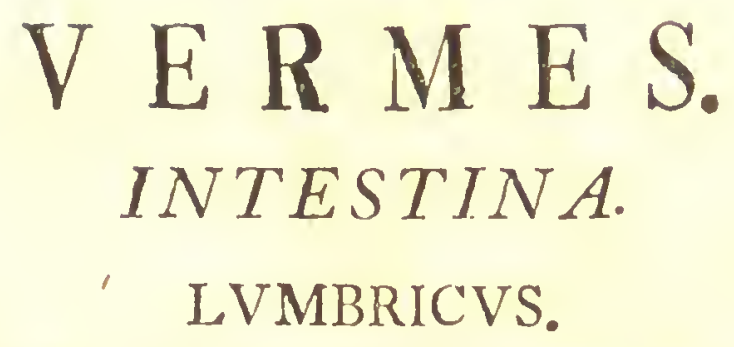

59. I V MBR ICVS terreftris trifariam retrorfum aculeatus. Syft. nat. 1076. [Murr. opufc. 2. t. 3. f. 1 - 5. Mull. verm. 1. vol.2. p.24. n. 157.] Lumbricus terreftris major. Raj. inf. I. Locvs: Terra abdita fpongiofa ubique. Manfuctus. PHARm. LVMBRICI exficcati, preparati, Pulvis, $\ominus, \Omega$, (). QYAL.

Vjitata. vis: antifpasmodica, temperans, diuretica. rsvs: Morbi fpasmodici, Arthritis fcorbutica. comp. Einpl. de ran. c. mercur; Vngu. nervin.

\section{HIR VDO.}

60. HIRVDO medicinalis depreffa nigricans, fupra lineis flavis fex: intermediis nigro arcuatis, fub. tus cinerea nigro maculata. Syft. nat. 1079. amcri. 7. p. 42. Bergm. act. Stockb. 1757. p.308. n.4.t.6. f. 1. 2. [Müll. verm. 1. 2. p.37. n. 167.] uocvs: Aqux ftagnantes, pigrx. Frequens, manfueta. PHARM: HIR VDO viva.

QVAL. Vitata.

Vis: topica.

vsvs: Hamorrhois.

COMP. 


\section{MOLLVSCA.}

\section{SEPIA.}

6r. SEPIA officinalis corpore ecaudato marginato, tentaculis duobus. Syjt. nat. 1095.

Sepia. Seb. mul. 3. t. 3.f. I- 4 .

zocrs: Oceanus.

Fera.

PHARM. SEPIAE OS. Frequens.

CVAL.

Vis: abforbens, adftringens.

vsvs: Gonorrhoa, I.eucorrhœa, Febr، intermittentes. comp. Pulv. dentifric.

\section{TESTACEA. OSTREA.}

62. OSTREA edulis tefta inæquivalvi femiorbiculata, membranis imbricatis undulatis: valvula altera plana integerrima. Syft. nat. 1488 .

Oftrea. Gefner. aqu. 2. p.33.

Locvs: Oceanus.

PHARM. CONCHAE, citrate.

QVAL. infipida, inodora, calcaria. Frequens.

vis : abforbens.

vis :

COMP.

Manfucta.

\section{MYTILVS.}

63. MYTILVS margaritiferus tefta compreflo-plana fuborbiculata bafi transverfa, imbricata tunicis dentatis. Sy/t. nat $\mathbf{1 1 5 5}$.

Matrix perlarum. Rumph. muf. 47. f. F. rocvs: Oceanus utriusque Indix.

Fera. PHARM. PERLARVM mater, cruda, preparata. QVAL. infipida, inodora, calcaria. VJitata. 
$v$ is: abforbens.

revs:

comp. Empl. Atictic, Vnga, nihili oculorum.

\section{HELIX.}

64. HELIX Pomatin tefta umbilicata fubovata ob. tufa decolore, apertura fubrotundo - lunata. $S y j t$. nat. 1244.

Cochlea Pomatia edulis Gefneri. Lift. exercit. anat. r. p. I62. t. $\mathrm{x}$.

x.ocvs: in Europa auftraliori freqtens, in hortis Suecia culta.

pharm. COCHLEAE Teftr, opercula.

QVAL. infipida, inodora, calcaria.

vis : abforbens.

Cicur.

vsve:

cons.

Vfitata.

\section{DENTALIVM.}

65. DENTALIVM Entalis tefla tereti fubarcuata continuata lavi. Sy/t. nat. I263.

Dentale lxve album aitera extermitate rufefcens. Li/t. bift. 4. f.2. n. 2.

Locvs: Oceanus circa Sueciam.

PHARM. DENT ALIOR VM Teft.

Fera.

QVAL. infipida, inodora, calcaria.

Rarior.

VIS : abforbens.

vsvs:

COMP.

\section{LITHOPHYTA. MADREPORA}

66. MADREPOR A oculata caulefcens tubulofa glabra flexuofa oblique fubltriata, ramis alternis, ftellis 
fellis immerfis bifariis. Syjt. nat. 1281. [Pall. $\approx 00 p h \cdot 308 \cdot n \cdot 179 \cdot]$

Corallium album vculatun oflicinarum. Baubin. bift. 3. p. 573. Seb. mul. 3. t. 116. n.12.

rocis: Mare Tyrrhenum.

Fera.

PHARM. CORALLIA alba, preparata.

erAl. infipida, inodora, calcaria.

Vfitata.

Vis: ablorbens, fubadtringens.

rste:

comp. Pulv. Cachect. pannon. rub. Empl. Atict. spec. cord. temperat. diamargarit. frigid.

\section{ZOOPHYTA. ISIS.}

67. ISIS nobilis ftirpe corallina rquali continua, Ariis obfoletis obliquis, ramis vagis. Syft. nat. 1288. [Pall. zooph.223. n. 142.]

Corallium rubrum. Baubin. pin. 366. Mar /igl. bift. mar. 108. t. $22-29$. 168. t. 40.

I.ocvs: Mare mediterraneum.

Ferc. pHarm. CORALLIA rubra, preparata, Tinctura. C.yL. infipida, inodora, calcaria. Vfitata. vis: abjorbens, fubaditringens.

vsvs: Prolluvia, Leucorrhoca.

comp. Specific. cephal. Pulv. bezoard. Sennert. dentifric. epil. marchion, de gutteta, pannon. rub. Conf. Hyacinth. Smaragd. Empl. flict. Spece. cord. temperat. de Hyacintl. diamargarit. Prigid. diamofeh. dulc. Trocb. de carabe.

\section{SPONGIA.}

68. SPONGIA officinalis foraminulata fubmo o dif formis tenax tomcritor. Sy/f. nat. 129s. TPull. $200 p 5.337 \cdot 23,234$. 
Spongia ad ufum proftantiflima, foraminibus exiguis pervia. Toum inft. 575.

Locrs: Mare mediterraneum.

pharm. SPONGIA, Cineres.

eyal. bibula, ficca.

vis: imbibens. Sief.

vsvs: Vlcus, Struma.

COMP.

\section{CORALLINA:}

69. CORALLINA officinalis fubbipinnata, articulis fubturbinatis. Syft. nat. 1304.

Corallina. Ellis. cor. 48. t. 24.

Mufcus coralloides, fquamis loricatus. Baub. pin: 364 .

Iocvs: Mare Atlanticum, Norvegicum. Manfueta. PHARM. CORALLINA.

QVAL. infipida, inodora, calcaria. Frequens. vis: abforbens, fubadftringens, anthelminthica. vsvs: Vermes.

comp. Pulv. ad vermes: Spec, diamercur. Mynf. 
R E G N V M

VEGETABIE. 



\section{CLASSISI.}
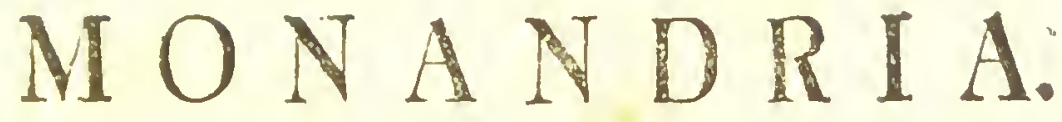

MONOGINIA.

\section{AMOMVM.}

70. AMIOMVM Zingiber feapo nudo, Spica ovata. Hort. cliff. 3. Fl. aeyl. 3. Lort. up. r. Sp.pl. r. facq. burt. I. t. 75 .

Infchi. Rbeed. mal. 1I. p.21. t. I2. Rumph. amb. 5. p. I56. t.66. f. I.

LOC. India orientalis; nunc Jamaica. Perennis, fera. PHARU. ZINGIBER IS COMMVM: Radiz zj.

A I. BI R.adix zi, nptima, utraque condita cum vel hile Brodio. QVAL. acris, aromatica, fervida, lubfiagrans.

Culinaris. vis: calefaciens, (recens eccoprotica), ftomachica, ftimulans.

vsvs: Colica, Diarnhea, Hyfteria, Tuffis ftomachalis.

comp. Andromach. Nithridat. Diafeord. Benedict. Hanech. Dialatyrium. Alhancial. Trocb. Agaric.

7x. AMOMVM Cardamomum fapo fimplicifimo brevifhimo, bracteis alternis laais. Fl. zeyl. 4.'Sp. pl. 2. [Kenig. monandr. $n$ g.]

Elettari 2. Rbeed. mal. It. p 9. t.6. Rumph. amb.5. p 152. t.65.f. . .

roc. Malabaria, Zeylona. Perenis, peregrina. PHARM. CARDAMOMI MINORIS ( rruetus)

non excorticauses, excorticatas. Semina, o . Rydr. acris, aromatica, fervida, fragrans. Culinaris. 
vis : ftimulans, calefaciens, nervina, pellens, $\mathrm{cm}$ a menagoga, aphrodifiaca.

vsvs: Anorexia, Morofis.

comp. Andromach. Mithridat. Benedict. Diafatyr. $\nabla$ Theriacal.

73. AMOMVM Gran. paradifi fcapo ramofo brevifimo. $S p . p l .2$.

Grana paradifi officinarum. Baub. pin. 413. Rsj. bift. 1205 . Barbara. zc.:. Madagafcar, Guinea, Zeylona, in umbrofis uliginofis ad radices montium. Perennis, peregrina. pHARM. PARADISI GRAN A (Semina). Cval. pracedentis $7^{1}$, fed acerrima. $\mathbf{v i s}$; ftimulans, calefaciens. vsvs: Paralyfis.

COMP.

Rarior.

\section{COSTVS.}

73. COSTVS arabicus. Hort. cliff. 2. Fl. zeyl. 5 . Hort. upfal. 2. Merian. Jim. 36. t. 36. Sp.pl.2. [Aublet. guilian.2.]

'Tfiana-kua. Rbeed. mal. II. p. 15. t. 8.

xoc. Malabaria, Zeylona, Brafilia, Surinamum. Perennis, fera.

phaRm. COSTI DVLCIS Radix $\boldsymbol{z}^{\beta}$. ARABICl Radix $\mathbf{z} \beta$.

QVAL. amaricans, aromatica, calida, fragrans. Rarior. Vis: ftimulans, calefaciens, diaphoretica, emme. nagoga, urinaria urina violacea odora. vsvs:

сомp. Andromach. Mithridat. Philon. Orvietanl. 74. MA.

62. GRAN. PARADISI Sem, alii A MOMI, alii MYRTI fpeciem ftatuunt.

73. COSTVM DVLCEM et ARAB ICVM ex eadem planta defumi plurimi fatuunt, eamque plantam officinalem cum nofira eandem elle verofinile eft. 


\section{MARANTA.}

74. MARANTA Galanga culmo fimplici. Sp. pl.2. Galanga major et minor. Rumph. amb. 5. p. 142.t.63. ıoc. India Orientalis, Philippinz. Perennis, fera. sHARM. GALANGAE MAJORIS Radix (ingratior, intus pallida).

MINORIS Radix (fortior, intus rufa). CyAl. acris, aromatica, amaricans, pungens magis Zingibere, fragrans. Preftans. vis: calefaciens, ftimulans, fomachica, emmena. goga, rec. fternutatoria. vsrs: Singultus! Vertigo! Vomitus navigantium,

Colica lochialis! Herpes. comp. Orvietanum, Aqua vitx Mattb. Pulvis na. falis:

\section{CVR CVMA.}

75. CVRCVMA longa foliis lanceolatis: nervis late. ralibus numerefiflimis. Roy. lugdb. 12. Fl.zeyl. 7. Sp.pl. 3 .

Amomum Curcuma. Facq. bort. 3. t. 4. Manjella - kua. Rbeed. mal. II. p. 2I. t. II. Rumpb. amb. 5. t. 67.

uoc. Amboina, Malabaria, Zeylona. Perennis, peregr. PHARM. CVRCVMAE Radix 3j.

RVAL. amara, fubaromatica, cibaria Indis. 'Rarior. ${ }_{\mathcal{W}}$ is: refolvens, pellens, emmenagoga, urinaria luteo. rinctoria.

vsvs: Icterus! Cachexia, Hydrops, Scabies! co.r. 


\section{KAEMPFERIA.}

76. KAEMPFERIA rotunda foliis lanceolatis petio. latis. Fl. weyl. 9. Sp. pl.3.

[Amomum fcapo nudo, fica laxa truncata. Berg. nuat. med. 4.]

Mulan-kua. Rheed. mal. Ir. p. r7. t. g.

L. Zeylona, Malabaria. Peremis, peregrina. PHA" M. " EDOAR IAE LONGAE Radix, Efferthe $\nabla, \Omega, 00$, Semiria.

CVAL. fubacris, aromatica, debile fragrans.

irequens, preftans.

vis: fimulans, calefaciens, nervina, ftomachica,

fudorilera, cmmenagoga, anthelninthica.

vsvs: Hyfteria, Afthria, Naufea! Vermes!

comp. Philonium. Acet. Theriacale. $\nabla$ Prophyla. ctiça. Aqua vitx.

\section{SALICORNIA.}

27. SALICORNIA berbacea patula, articulis apice compreflis emarginato-bifidis. Fl. Juec. I. Sp. pl 5. Bafter. fubfec. 2. p. 105. t.50. Fl. dan. 303. Pall. it. I. p. 479. tab. A. f. .

Kali geniculatum majus I. Baulb. pin. 289.

ıоc. Europa. Anmu, maritima, manfueta. PHARM. SODA, $\theta$.

QVAl. falfa.

Culinaris, rarier. Vis: Atimulans, diuretica, emmenagoga. (Sal alcali fixum corrofivum).

vsys: Scabies, Abfcellus, Scorbutus, Hyperfarcofis. comp. Sajpo verietus, Sal Seignette, Fel vitri.

CLAS-

26. Ex hac planta ZEDOARIAE ROTVNDAE rad. itidem colligi vulgo flatuitur. Sed hree in nonris oflicinis non proftat.

77. SODAM e variis plantis maritinis fallisque conficinn varii; uti ex Mesembryanthema degyptii; Chenupudio Hifpa-

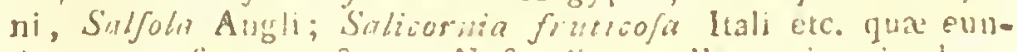
denis tere ufum preftant. Noftratibus nulla aptios in huse ufum planta, quam dicta. 


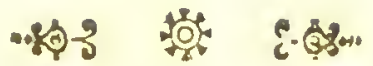 \\ CLASSIS II. \\ D I A N D R I A. MONOGTNIA. JASMINVM.}

78. JLSAINVM officinale foliis oppofitis pinnatis: foliolis diltindis. Hort. cliff. 5. Hort. up 5. 5. Sp. pl. 9. Yasminum vulgatius hore albo. Baub. pin. 397.

r.oc. India orientalis. Arbufcula, manfueta. PHARM. JASNINI (Flonm) (O).

QI.X. fragrans.

Extorna.

ris: narcotica, anodyna, uterina.

viss: Dyftocia.

coms. Pulvis Cyprius, Odoramenta.

\section{OLEA.}

79. OLEA europea foliis lancenlatis. Ilort. cliff. 4 . St.pl. in.

Olea fativa. Bauth. pin. $47^{2}$.

r.uc. Iifpania, Gallia, I:alia (cœlo temperato). Arbor, manjueta, ferilis. BHARs. OLIVAE (Fructus) condita, (O). (1).r. (1) pingue, blandum, infipidum, inodorum; Condit. aufterx.

Cillinaris.

ris: (1) obtundens, cmolliens, leniens, relaxans. Condit. tonica, flomachica.

isvs: Venena! [Mleuritis, Nephritis, Enteritis]

i) Tenteria, Tunesmus, Colica, Rheumatsmus. 'iulis, Mlorfura canis rabidi.

cons.

$$
\text { C } 4 \text { VERO. }
$$

7. I Hi flores fuccedanei funt NYCTANTHIS, cumqne nec JAS-

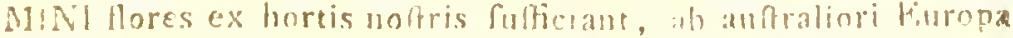

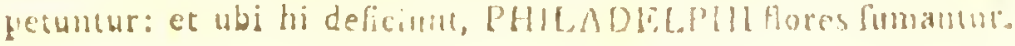




\section{VERONICA.}

80. VERONICA officinalis fpicis lateralibus pedunculatis, foliis oppofitis, caule procumbente. Fl. fuec. 12. Sp. pl. I4. Fl. dan. t. 248 .

Veronica mas fupina et vulgatiflima, Bauls. pin. 252. цoc. Europx fylva aridx. Perennis, vulgaris. pharm. VER ON ICAE Herba; Conjerva, Symupus, $\nabla$. Ral. fubltyptica. Trita. vis: fubadfringens, vulneraria, tonica, pectoralis, vsvs: Cachexia, Afthma, Tullis.

COMP.

31. VERONICA Beccabunga racemis lateralibus, fo. liis ovatis planis, caule repente. Fl. Juei. 14. Sp. pl.x6. Fl. dan. t. 51r.

Anagallis aquatica major (et minor), folio fubrotundo. Baub. pin. 252.

toc. Europx fcaturigines. Perennis, frequens. pharm. BECCABVNGAE Herba, Conferva, Aqua. QVAL. fubinfipida, inodora, oleracea. Rarior, Juperfnu. vis: diuretica? difcutiens?

vsvs: Scorbutus.

COMP:

\section{GRATIOLA.}

32. GRA TIOLA offlimalis floribus pedunculatis, fo. liis lanceolatis ferratis. Sp. Hl.24. Fl. dan. t.363. Gratiola centauroides. Baub. pin. 279.

zoc. Europa auftralior. Perennis, paluftris, mansueta. PHARM. GRA TIOLAE Herba 3 j.

QY.X. amara, naufeofa.

Eximia, couta. vis: purgans, vomitoria, emmenagoga, anthelminthica.

vsvs: Dyfenteria, Hydrops.

comp. Enemata. 


\section{VERBENA.}

8. VERBENA officinalis tetrandra, fpicis filiformibus paniculatis, foliis multifido-laciniatis, caule folitario. Hort. cliff. 11. Fl. fuec. 30. Sp. pl. 29. Fl. dan. t. 628 .

Verbena communis flore creruleo, Baub. pin.269. zoc. Europa auftralior, Scania. Amma, cicur. PHARM. VER BENAE Herba, $\nabla$. cyal. fubinfipida, inodora. Dubia, exoleta. vis: adfringens, vulneraria, vsvs: Ophthalmia? Magia? COMP,

\section{MONARDA.}

84. MONARDA fifulofa capitulisterminalibus, caule obtulangulo. $S p \cdot p l .32$.

Monarda mollis. Amoen. acall. 3. p.399.

ıoc. Canada.

Perennis, cicur. PHARM. MONARDAE Herba. QYAL. Ppirans, amara.

vis : refolvens, nervina, tonica.

Imuftata, eximia. vsvs: Febres intermittentes!

COMP.

\section{ROSMARINVS.}

85. R OSMARINVS officinalis. Hort. cliff.r4. Hort. upf. Ir. Sp.pl. 33 .

Rosmarinus hortenfis anguftiore, et fpontaneus latiore folio. Baub. pin. 217 .

soc. Gallia narbonenfis, Itipania, Fruter, manfuetus. PHARM. RORIS MARINI IHSPANICI f. IIORTFissis Herba, $\nabla$, o , Semina.

ANTitos Flores, (onnferou, $\Omega$ (aqua Reg. I long.)

evar. fpirans, calida. Ifitata, beroica. 
ris: excitans, nervina, tonica, cephalica, flomachica, refolvens, emmenagoga.

riss: Syncope, Afthma, Hyfteria, Chlorofis, Apoplexia, Tertiana.

cosp. Mel antholatum,

\section{SAR. VIA.}

\$6. SALVIA officinalis foliis lanceolato-ovatis integris crenulatis, floribus fpicatis, calycibus acutis. Hort. cliff. 12. Hort. upf. 10. Sp. pl. 34 .

Salvia major. Baub. pin. 237 .

zoc. Italia, Natbona. Peremis, olitoria, cicur. pharar. S A L V I A E Herba, $\nabla, \Omega$, 0 , Fl. Sem. QVAL. Spirans, amaricans. Frequens, preftnus. vis: adfingens, tonica, nervina, ftomachica, uterina.

vsvs: Timor, Languor, Leucorrlica, Senectus. COMP. 娄 fternutatorius viridis.

87. SALVIA Horminum foliis obtufis crenatis, braGeis fummis fterilibus majoribus coloratis. Virid. cliff. 4. Sp.pl. 34 .

Horminum fativum. Baub. pin. 238.

ı.oc. Apulia, Cracia.

FHARM. HOR XINI Semina.

QVAl. fpirans, amaricans, mucilaginofa.

vis: invifans.

voss: Ophthalmia.

coup.

\$3. SAIVIA Sclarea foliis rugolis cordatis oblongis villofis ferratis: bracteis coloratis calyce longioribus concavis acuminatis. Hort.up/. 10. Hoiminum Clarea dictum. Baub. pin. 208.

luc. Sylia, Italia. Biemis, olitoriu, manfueta. 


\section{DIANDRIA. TRIGYNIA.}

pharir. SCLAREAE (GALLITRICHI) Herba. Q'AL. fpirans.

Rarior. VIs: fortior Salvia officinali, fimulans, exhilarane,

fermutatoria, refolvens.

vsrs: Lizucorrhoa, Hyfteria, Colica.

comp.

\section{COLLINSONIA.}

89. COLLINSONIA canadentes, Hort. cliff. 14. t.5. Sp. pl.39. Schepf. mat. med. \%.

roc. Virginia, Canada. Perennis, manfueta. PHARM. COLLINSONIAE Radix.

eVAL. nidorola.

VIS :

vsvs: Colica lochialis.

conp.

\section{TRIG $Y N I A$. PIPER.}

90. PTPER nigrum foliis ovatis fubfeptinerviis glabris, petiolis fimplicillimis. Fl. zeyl.26. Sp. pl. 40. Malago - Codi. Rheed. mal. 7. p. 23. t.12. loc. India orientalis. Frutex, peregrinus. PHARA: 1'IPERIS NIGRI Fructus, A l. B I Fructus.

WVAL. acris, aromatica, fervida. Culinaris. Vis: calcfaciens, flimulans, ftomachica, fingultuofa, fernutatoria, aphrodifiaca, exanthenatica. rsis: Tertiana, Odontalgia, Prolapfus uvulx, Pediculi.

conp. Andromach. Mithridat. Diaphœnic. El. bacc. laurị. 


\section{DIANDRIA. TRIGYNIA.}

9r. PIPER longum foliis cordatis petiolatis feflilibusque. Fl. zeyl. зо. Sp. pl. $4 \mathrm{I}$.

Cattu - Tirpali. Rbeed. mal.7. p.27. t. I4.

L.oc. India orientalis.

PHARM. PIPER IS LONGI Fructus.

Frutex, peregrinus. QVí. acris, aromatica, fervida.

vis: calefaciens, ftimulans, ftomachica, fingultuola. vsvs: Tertiana, Flatulentia.

COMP.

93. PIPER Cubeba foliis oblique ovatis oblongisve venolis acutis, fpica folitaria pedunculata oppofitifolia, fructibus pedicellatis, Suppl.pl.90. Syft. veg. 14. p. 74 .

Cubebe vulgaris. Baub. pin.412. Barbara. Loc. Java.

pharm. CVBEBAE Fructus, 0 .

QVAL. acris, aromatica, fervida.

vis: flimulans, calefaciens, carminativa, fralagoga. vivs: Vertigo, Morolis.

COMP. 


\section{野 \\ CLASSIS III. \\ T R I A N D R I A. MONOGYNIA. \\ VALERIANA.}

93. V A LER IA N A offucinaiis floribus triandris, foliis:

omnibus pinnatis. Fl. fuec. $34 . \mathrm{Sp}$. pl.45. Fl. dan.

t. 570 .

Valeriana fylveftris major. Baub. pin. 164.

Ioc. Europæ prata paludola. Perenmis, vingaris. PHARM. VALERIANAE SYLVESTRIS (MI.

NORIS) Radix $\mathbf{z}^{\mathrm{ij}}$.

QVal. hircofo-felina, amaricans. Preftans. vIS: narcotica, antifpasmodica, fudorifera, diure.

tica, purgans, [emmenagoga, anthelminthica.] vsv's: Epilepfia, Convulfiones, Hyłteria, [Hemicrania, Vifus hebetudo,] Hxmoptyfis, Vlcus.

COMP.

44. VALERIANA Phu floribus triaridris, foliis caulinis pinnatis, radicalibus indivifis. Hort. $u p \int .13$. Sp: pl. 45 .

Valeriana hortenfis. Bauh. pin.164.

ıoc. Alfatia, [Ruflia.] Perennis, cicur. PHARM. PHV (VALERIANAE MAJORIS)

Radix, Herba.

QYAL. antecedentis.

Exoleta.

Vis: antecedentis, vulneraria.

vsis: antecedentis.

romp. Andromach. Mithridatium.

95. VALERIANA celtic a floribus triandris, foliis ov to - oblongis obtufis integerrimis. $S p . p l .46$.

Nardus celtica diofcoridis. Baub. pin. 165. 
Loc. Helvetix, Carinthix, Styrix, Liguria," Ge nua Alpes. Perennis, peregrina. PHARM. SPICAE CEI, TICAE Radix, Flores. QVAL. fragrans, acris, aromatica (plantx fere errarcid $x$ ).

Rairior.

vis: antilpasmodica, diuretica, 'anthelminthica. vsvs:

comp. Andromach. Mitlridat. Empl. Meliloti.

\section{TAMARJNDVS.}

96. TAMARINDVS indica. Rumph. amb. 2. p 90 . t.23. Hort. cliff. 18. Sp. pl. 48. Gacq. amer. 10. Balam - pulli. Rbeed. mal 1.p.39. t.23.

Loc. India utraque, Aegyptus, Arabia. Arbor, fera. pharm. 'TAMAR INDI Fructus, Pulpa $z^{3}$ ij.

RVAL. acida, fubnauleola. Eximia. vis: eccoprotica, refrigerans.

vsvs: lebres phlogiflicx, putridx, [Diarrhœa, Dyfenteria, Iclerus.]

comp. Elect. cathol. Elect. lenitiv: Conf. hamech.

\section{R O C V S.}

97. CR OCVS fativus: officinalis fpatha univalvi radicali, corollix tubo longiflimo. Sp. pl. 50 .

Crocus lativus. Bath. pin. 65. Crocus alpinus autumnalis, C. B.

roc. Helvetix, Pyrenzorum, Lufitanix, Thracix Alpes.

Perennis, cicur. PHARM. CROCI ORIENTALIS (Stigina) Biß. BR IT A N'NICI (Stigma) pulvis $5 \beta$, Tinct. Эiß, Extractum gr. XV. QVAL. fragrans, fubaromatica, luteo - tinćtoria.

Culinaris, beroica. vis: ainodyria, antifpasmodica, hypnotica, exinila. rans, refolvens, uterina, pellens, emmenagoga, exanthematica, urinaria, lactaria. 
rsre: Colica lochialis, Vomitus, Tullis, Dyfenteria, Dyfuria, Dysmenorhoea. Dyslochia, Afthma, lcterus, Contufura, Ophthalmia.

eomp. Andromach. Mithridat. Philon. Benedid. Orvietan. Hiera. Conject. hyacintin; to Comitill. Trosh. Carabe; Laudan; Elix. propriet; Pil. anmon; Pil. rufi: Pil. cynoglon; (O) Scorp. mag. Vhg. aureum. Empl. Oxycroc. Limpl. melilot. Empl. galban. Empl. diachyl. compos.

\section{IR IS.}

9S. IRIS forentina corollis barbatis, caulc folits altiore fubbitloro, floribus felinibus. $S p \cdot p l .55$. Mill. ic. 154. [Thuizb. diff 4.9.]

Iris alba Horentina. Bumb. pir. 3T.

LOC. Italia.

Perenanis, manfretic.

PHARIr. IREOS FLORENTINAE Fadix.

QVAc. fragrans, acriufcula, amaricans. Frequens. vis: incidens, expectorans, errhina, eccoprotica. vsus: Tutis, Hytrops. [Tomina infant.],

comp. Andromach. Species Dialreos. Tiroch. bec. chici. 宩 ftermutorius. Vnisu. pomatum.

99. In IS germmica corollis barbatis, caule foliis altiore multitloro, Horibus inferioribus peduncula. tis. Hort cliff. 18. Hort. up. I6. Sp. pl. 55 . ['Thunb, dif 4. II.]

Iris vulgaris germanica f. fylveftris. Baub. pin. 30. t.oc. Germanix, Galiize clivofa. Cickr. Harm. IREOS NOSTRATIS Radix, Feculc. (WAL acris.

Heroica.

vis: diurctica! hydragoga, fubenetica, emmenzgnga, crihina.

vsvs: Hydrops! Oedema.

COMP. 
100. IR IS Pseudo-Acorus corollis inberbibus, petalis interioribus ftigmate minoribus, foliis enfiformibus. Fl. fuec. 37. Sp. pl.57. Fl. dan. 494.

Acorus adulterinus. Baub. pin. 34.

Loc. Europx paludes. Perennis, vulgatifina. PHARM. ACORI PALVSTRIS Radix:

QVAL. infipida, fubrtyptica.

Rariờ.

vis : adfringens,

vsvs: Siomacace.

Comp. Elect. Diacori.

10I. IK IS foetidifimn corollis imberbibus: petalis in terioribus patentiflimis, caule uniangulato, foliis enfiformibus. Sp: pl. 57. Hudf. angl.14.

Gladiolus foetidus. Baub. pin. 30.

zoc. Gallia, Anglia, Italia.

Perennis. PHARM: XYRIDIS f. SPATHVLAE FOETIDAE Radix.

CYAL. tetra, fotida, acris.

Exoleta. vis: narcotica, antifpasmodica, purgans, pellens. vsvs: Hylteria, Scrophula.

COMP.

102. IRIS tuberofa corollis imberbibus, foliis tetragonis. Vir. cliff. 6? Sp. pl. 58 .

Colchicum radice ficcata alba. Baqb. pin. 69.

r.oc. Syria, Arabia.

Perennis, manjueta.

PHARAI. HERMODAC'TYLI Radix $z^{\mathrm{ij}}$.

QVAL. inodora, dulcefcens, acris.

Dubia.

$\mathrm{v}$ Is: purgans, mundificans.

vsvs: Rheumatismus, Amaurofis.

comp. Benedict. $f$ Artheticus, Extr: panchymagog.

CYPE.

120. Rad. HE RM O D A C TY LI. Magnus olim Botanicus Tournefort, qui plagas orientales ipfemet peragravit, hujus matrem effe lridem tuberofasm; fed recentiores, ut Millerus et alii, volunt effe Colchicun illyrisum, idem confirmante For'sk fhollio, [Conf, Spiehm. mat, med, p. 635.] 


\section{CYPERVS.}

103. C Y PER VS longus culmo triquetro foliofo, unbella foliola fuprakcomporita, vedunculis nudis, fpicis atemis Roy. lugdl. 50. Sp. pl. 67.

Cyperus odoratus, ratice Jonga 1. Cyperus offici-

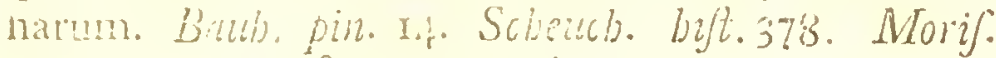
bift. 3. p. $237 \cdot f \cdot$. . . i. 12. f. I3.

Loc. Gallix, Italix patudes. perennis, manjueta. PHARM. CYPERI LONGI Radix.

Qyal. fragrano, amarican, calida.

Exoleta.

VIs: diuretica, emmenagoga.

rsys: Heera uteri, vefica, oris.

cons.

104. CYPER VS rotumins culmo triquetro Rubnudo, unbella decompofita, fpicis aitunis linearibus. Fl. zeyl. 36. Sp.pl. 67.

Cyperus ortentalis major. Baub. pin. 13. theatr. 208. idorif. bift. 3. p. 236. t. II. f.2.

¿oc. legyptus, Syria. Perennis, peregrina. PHARI. CYPERI ROTVNDI Radix.

QN+. fragrans, amaricans, calida. Exoleta, fuperfun. Ys: antecedentis, debilior.

viss:

coup. Trocb. capparidis; Empl. meliloti.

\section{DIG NIA. SACCHARVM.}

105. SACCHARVM officinarum Horibus paniculatis, foli:s planis. Hort. cliff. 26. Hort. upj. rg. Sp.pi. 79. Arundo faccharifera. Bathls. pin. 18. Slom. jam. 1. p.108. t.66. Rumpp. amb.5.p.186. t.74. f. I.

I.OC.

104. CYPERVS rokudus fi idem cum Cypero orientali, ra-

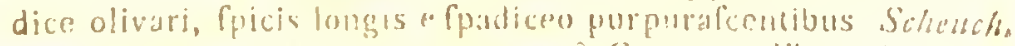

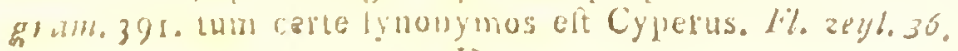


s.oc. India utriusque inundata. Perennis, fera. PHARM. SACĆHARVM Canariene, candidum, album, rubrum, fintinacerun, album, grifeum, Penidiun, Perlatum, Rubrums. Syrupus. evar. dulciflima, inodora, mitiflima. Culinaris, ufitatifima. - vis : edulcorans, relaxans, calefaciens, expectorans, vulneraria, antileptica, nutriens. vsvs: [Raucedo, Tullis, Pannus.]

comp. Omnes Confectiones, Condita, Conferva, Syrupi.

\section{PHALARIS.}

106. PHALARIS canarien/is panicula fubovata fpiciformi, glumis carinatis, Hort. ups. 19. Sp. pl.79. Scbreb. gram. . p. 83. 't. Io, f. 2.

Phalaris major, femine albo. Baub. fin.28. Morif. bift. 3. p.186. ..8. t.3. f. 1.

roc. Canarix, Hetruria, Hifpania. Anmua, cicenr. pharm. CAN $\Lambda$ RIENSE Semen.

QVAl. farinacea.

vis : nutriens.

vsve: Aviculis.

COMP.

Superfura.

\section{PANIC VM.}

107. PANICVM miliacenn panicula laxa flaccida, foliorum vaginis hirtis, glumis mucronatis nervofis. Hort. cliff. 27. Hort. ups. 20. Sp.pl. 86.

Milium femine luteo et albo. Baub. pin. 26. theatr. 502. I.OC. India.

pharm. MILII Semina.

eval. farinacea, infipida. Culinaris, fuperfua.

vis: nutriens, flatulenta. Annuninl, cicur.

viss:

comp. 


\section{A V E N A.}

108. A VENA fativa paniculata, calycibus difpermis, feminibus lavibus. Hort. cliff.25. Hort. ups. 20. Sp. pl. I18.

Avena nigra et alba. Baub. pin. 23. thentr. 469. zoc. Alia. Annuc, cerealis, frequentiffima. FHAR M. A VENA excorticata.

CrAL. farinacea, infipida. vis: refrigerans magis quan Hordeum.

Vjetatifima. vsis: Febres.

comp.

\section{HORDEVM.}

rog. HORDEVM diftichum flofculis lateralibus nkafculis muticis, feminibus angularibus imbricatis. Hort. upf.23. Sp.pl. I25.

Hordeun diftichum. Baub. pin. 23. theatr.440.

LOC. Annum, cereale, vulgare. PHARM. HORDEVM mundatum, excorticatum, perlatum. Acetrmonlgare, $\Omega$, (deftillatum). Cerevifa antiqua, gedanendis (Pryfling.) QVAl. farinacea, infipida. Culinaris. vis: mutriens, refrigerans. Aceti refolvens, refrigerantiflima.

rsvs: + Inflammatio, Cerevif. Contufura. cosp. Loboc hordeatum. Syripus acetolus.

\section{TRITICVM.}

INO. TRITICVM hybernum calycibus quadrilloris ventricofis lavibus imbricatis lubmuticis. Lurt. upp. 2I. Sp.pl.126.

Triticum hybernum, arifis carens. Baib. pin.2r. tbeatr. 352 .

I. C. .

Anmumn, cereale, frequentifinnum.

$D_{3}$

1'HARM 
PHARM. AMYLVM, Pulvis Cyprins. QVAL. farinacea, infipida.

vis : nutriens, acefcens, mucilaginofa.

vsvs: externus.

COMP.

III. TRITICVM repens radice repente, foliis planis. Fl. flec. r4. Sp.pl. 128. Sclreb. gram. 2.p. 24. t.26. Fl. dan. t. 748 .

Gramen caninum repens vulgatius. Morif. bift. is. p. I18. f. 8. t. . . f. 8 .

Loc. Europx nofre iuderata. Perenne, vulgatifinum. PHARM. GR $\Lambda$ M INIS Radix, $\nabla$. Qvar. dulcefcens, inodora. vis: nutriens, aperiens, diuretica, mundificans, anthelminthica.

vsvs: Sterilitas, Obfiuctio vifcerum. comp. Radices aperientes minores.

111. Radix GRAMINIS apud nos ubique defumitur a Tritico repente; at in Italia ex Panico datfylo, tefte Seguicrio. 


\section{8 \\ CLASSISIV. \\ TETR A N D R I A. MONOGINIA. \\ SCABIOSA.}

112. SCABIOSA Succifa corollulis quadrifidis xqualibus, caule fimplici, ramis approximatis, foliis lanceolato-ovatis. Fl. fuer. IIg. Sp. pl. I 42. Il. dan. t. 279 .

Succifa glabra. Baub. pin 269 .

Loc. Europap prata humidifcula. Perennis, vulgatif. PHARM. MORSVS DIABOLI Radix, Herba. cir.. ral. dulcis, fubamara. Rarior, Juperflua. $v_{1}$ : fubachlingens.

rsvs: Angina.

conp.

1j. SCAHIOSA arvenfis corollulis quadrifidis radiantibus, foliis pimatifidis incifis, caule hilpido. Fl. fuec. 117. Sp. pl. 145. Fl. dan. t. 447.

Scabiola pratenfis hirfuta. Baub. pin. 269.

unc. Furopre prata, agri. Perennis, vulgaris. PHAR.S. SCABIOSAE Radix, Herba, $\nabla$, Syrup. Fl. und. debilis.

Vitata.

Vis : raci. diluens, alexiteria, becchica, exanthematica. vsve: Scabies?

comp.

\section{HEDYOTIS.}

14. HEDYOTIS Auricularia foliis lanceolato - ovatis, floribus verticillatis. Fi. $\approx e y l .64 . *$ Sp.pl 147 .

J) $\hat{3}$ Vale-

114. Asricularia hrec, qure a Doleo pharm. roo. tamquam fuecificum extulliur in furditate, nondum introducta eft iun offici-

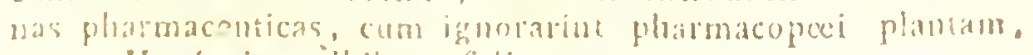
quam Marlonizs athibere folitus erat. 
Valerianella paluftris, foliis nervofis oblongis, Burm. zeyl. 227. t. 108. f. x.

toc. Zeylona. PHARM. AVRICVLARIAE Hẹba, QVAL. fragrans.

Frutex, percgrinus. VIS:

vers: Cophofis.

COMP.

Ignota.

\section{ASPER VT,A.}

15. ASPERVIA odırata foliis odtonis lanceolatis; Horum falciculis pedunculatis. Fi. fuec. 12r. Sp. pl. 150. 1\%. dan. 1.562.

Afperula [. Rubeola montana odorata. Baub. pin. 334 . ¥.оc. Europa lytur unbrofx. Perennis, frequens. PharM. MATRISILVAE Herba.

QVAL. ambrofiaca.

Rarior.

VIs: diluens, diuretica, exanthematica.

vsvs: Exanthemata, Scabies, Icterus.

COMP.

\section{G A L I V M.}

II6. GALIVM verum foliis octonis linearibus fulcatis, ramis Horiferis brevibus. Flor. Juec. 123. Sp. pl.155. Gallium luteum. Banı. pin. 335 .

цoc. Europe prata. Perennis, vulgatiflima. PHARM. GALLII LVTEI Herba (Coma potius). UYAL. fragrans.

vis: coagulans lac.

vsvs: Hyfleria Jorf. Epilepfia, Hamaturia.

COMP.

Rarior.

\section{R T $T^{\top} \mathrm{I} A$.}

I17. RVBIA timctorm 'foliis annuis, caule aculeato. Hort. ulf. 35 Hort uf. 23. Sp pl 158. Mant.330. Rubia tinciorum fativa. Bazb. tin. 33. 
ıoc. Monfpelii, Danubii prata. Perennis, manfueta. PHAR. RIHLE: 'TINCTORVM Radix, Tinceura. Mra. Arptica, rubra.

Rarior. Vis: adilngengs, pellens, diuretica, tinctoria,

urinam et ofia tingens.

rsvis: Contulira, [Rachitis.]

comp. Raäices $V$ aperientes minores, $V$ ngu. potabile.

\section{PENAEA.}

II8. PFNAEA mucronala foliis cordatis acuminatis. Sp. p!. i6z.

Tithymali myrenitis fpecic arbufcula xthiopica, folin parro c lata bait in acutifimum mucronem fubito definente. Pluk. mant, 183.

1.0C. Aethiopia. Fintex, peregrinus. PHARM. SARCOCOLLAE Gummi. ortL. Gummi-refina, rufefcens, friabilis, fubdulcis, amaricans, acris, inodora.

Rarior.

vis: vulneraria, puigans, noxia.

rsvs: Vulnera, Ophthalmia.

cump. Empl. Opodeloch.

\section{PLANTAGO.}

rig. PI.ANTAGO major foliis ovatis glabris, fcapo tereti, Snica flofculis imbricatis. Hort. cliff. 36 . Fi. free. 129. Sp. pl. 163. Fl. dan. t. 46r.

Plantago latifolia major. Bauh. fin. I89. i.co. iuropa.

Perennis, onlgatifina. HHAR. HLANTAGINIS LATIFOLIAE Radix, Herba, $\nabla$, Semina.

(M). inspida, herbacea, fubfyptica.

Rarior.

vis: adfrimgerts, vulneravia.

rovs: Vlecra, Diamhoca, [Tertiana.]

coni. Gargarilmata.

1) $4 \quad 120$. PLAN-

19. [PIANTAGO major, mediar et Inncrolata fobi invicem abs-

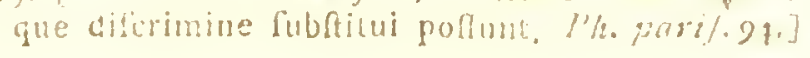


120. PLANTAGO Pfyllim caule ramofo herbacco, foliis fubdentatis recurvatis, capitulis aphyllis. Hort upl 28. Sp.pl.167. Pfyilimm majus erectum. Baab. pin rgr. loc. Europa antralis agri arenofi. Amma, cicur. PHARa. PSYLLII Semina, Mucilago.

Qya. mucilagino a.

Rarior. vs: invilcans, lubricans, refrigerans, confolidans, emolliens?

rsus: Ophthalmia, Dyenteria, Raucitas, comp. Gargarilimata, Clylmata.

\section{SANGVISORBA.}

I21. SANGVISORBA offuinulis lpicis ovatis. Hort. cliff. 39. F\%. fuec.37. Sp. pl.s6g. F\%. dar. t.97. Pimpinella languiforba major. Baubs. pin. x6o.

coc. Europie auftralioris, prata. Peremis, cicur. PHARM. PIMPINELLAE ITALICAE Radix, (non Herba, non Semina).

Qril. Ayptica.

Y Is: adftringens, tonica,

vsrs: Hamorrhagia, Dy̧fenteria, Hydrophobia Palmar.

cons.

Exolcta, fuperfua.

\section{FAGARA.}

122. FAGARA Pterotn foliolis emarginatis. Sp. pl. 172. Jauro adfinis, jasmini folio alato, ligni duritie fer. ro vix cedens. Sloan. bi/t.2. t. I62. f. I. Loc. Philippinarum, Iamaicx campeftria.

PHARM. FAGARAE Paccr. Albor, peregrima. QVAL. acriulcula, aromatica, bifida, cava. Exoleta, fuperfua.

120. [PSYLLII fern, e Plineng. Caynope colligenda effe Beig. ] 


\section{TETRANDRIA. MONOGYNIA.}

rs: caletaciens, exliccans, ftomachica.

vsus: Anorexia.

cosp. Compotitiones Arabum.

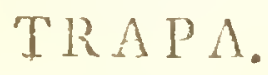

123. TRAPA notans. Fi. fiec. 140. Sp. pl. 175.

Tribulus aquaticus. Banb. pin. 194. Carr. epit.715. r.oc. Europia aufralis aqua limolix. Infrequens. pHAR. AQVATICAE NVCIS (Fructus). एal dulcis. farinola. Exnieta, fuperfua. vis: nutriens, adfingens, obfipans.

vsve: Mlenritis.

comp. Vngz. agripp.

\section{DORSTENIA.}

124. DORSTENTA Contrajerva fapis radicatis, fo. liis pinnatifido - palmatis Ferratis, receptaculis quadrangulis. $S p \cdot p l .176$.

Drakena radix. Cluf. exot. 83.

Loc. Heru, Nexico, Vera crux, Inf. Vincentii. PHARM. CONTRAJERVAE Radix. Perennis, peregrina. QHA. aromatica, flubfregrans, amaricans.

Vjitatu, preefens.

ris: adfringens, ftomachica, alexiteria, fudorifera. rsss: Febres omnes, Exanthemata, Dylenteria. Conp. 妾 Cominilix.

\section{SANTALTM.}

125. SANTALVM album. Sp. pl. 497. Rumpb. amb. 2. p. 42. i. 11 .

1) 5 Sall.

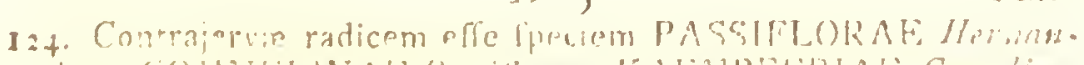

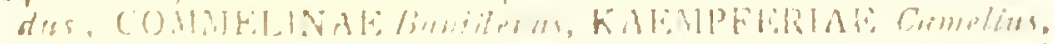

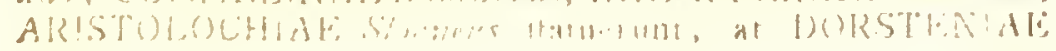

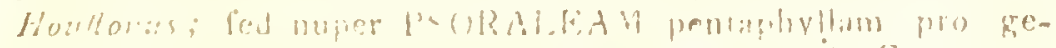

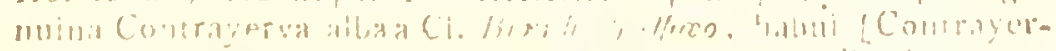

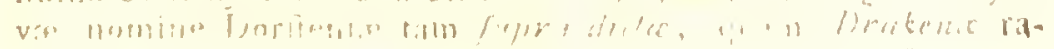

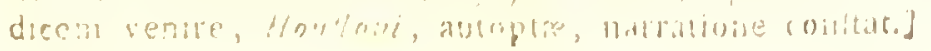




\section{TETRANDRIA. MONOGYNIA.}

Santalum verum. Breyn. icon. 94.t.5. f. I.

roc. India, Siam, Timor, Solor. Arbor, peregrina. PHARM. SANTALI CITRINI lignum.

$$
\text { ALbI Lignum. }
$$

QVAL. fragrans, fubambrofiaca, amaricans.

Exoleta, dubia, fisperfiua.

vis: fubrenenata, fynochizans, tonica, fudorifera. vsvs: Butillationes.

comp. Confect. Alkermes. Pulv. abort.

\section{CAMPHOROSMA.}

326. CAMPHIOROSMIA monjpeliaca foliis hirfutis linearibus. $S p . p l .178$.

Camphorata hirfuta. Bauh. pin. 436. Buxh. cent. I. p. I8. t. 28. f. I.

zoc. Juropa auftralis, Tataria.

Annua.

THARM. CAMPHORATAE Herba.

QVAL. Subaromatica.

Tis: nervina, diuretica, fudorifera, relolvens, vulieraria, cephalica.

viss: Hydrops, Hyfleria, Coryza, Leucorrhœa, Afthma! Rheumatifmus!

COML.

\section{ALCCHEMILIAA.}

127. ALCHEMLLLA vulyaris foliis lobatis. Fl. Suec. 141. Sp. pl. 178. Iil. dan. t.693.

Alchimilla vulgaris. Baub. pin. 319.

soc. Juropa palcua ficciora. Peronnis, oulgatiffina. гнarm. ALCHIMILI.AE Radix, Herba, Aqua. खN.. Alyptica, inodora. Rarior. vis: vulneraria, fophilicans.

vsre: Dyfenteria.

eON1? 


\section{DIGINIA. \\ CVSCVTA.}

I28. CVSCVTA eqropea floribus feflilibus. Fl. Juec: 144. Sp. pl. 180. Fl. dan. t. 199.

Culcuta major. Baub. pin. 219.

soc, Europe noftratis Linum, Humulus, Vrtica;

Cannabis.

PHARM. CVSCVTAE Herba.

Cr.A. amaricans, acriufcula, inodora. Annu, vulgaris.

ris: laxans, [Atimulans, incidens, aperiens.]

vsvs: Febres intermittentes. [Obftructiones vifc.]

COMP.

29. CVSCVTA Epitbymm floribus feflilibus quin. quefidis, bracteis obvallatis. Sy/t. veg, 167. Loefl. it. 216 . Fl. dar. t. 427 .

Epithymum f. Cufcuta mmor. Baub. pin.219.

toc. Europre auftralis Thymus. Anmsa, monfueta. PHARM. EPITHYMI Herba.

Qval. odor et fapor fere Thymi, fed debilior.

vis: precedentis,

Dubia.

vsve:

comp. Conf. hamech.

\section{TETR AGINIA. \\ ILEX.}

T3o. IJEX Caffine foliis ovato-lanceolatis ferratis, Hort. cliff. 40. Sp.pl. I8I.

Aquifolium carolinenle foliis dentatis, baccis rubris. Catesb. car. I. p.3I. t.3I.

soc. Carolina, Virginia.

Aibor, manfueta.

THARM. CASSINAE Folia.

orai. fubaromatica, inodora.

Imijitata.

ris: oxpectorans.

vsis: Variola!

COMP. 


\section{CLASSIS. V. \\ PENTANDRIA. \\ MONOGYNIA. \\ LITHOSPERMVM.}

I31. LITHOSPERMVM officinale feminibus lavibus, corollis vix calycem fuperantibus, foliis lanceolatis. Roy. lugd 405. Fil. Juec. 159. Sp. pl.189. Litholpermum majus erectum. Baub. pin. 253. 1.oc. Europa aufralior. Perennis, cicur. PHARM. I.T'THOSPERMI Semina.

(2vis. infipida, inodora, offea. Exoleta, fuperfuta. vis: ablorbens, adflingens, diuretica?

vive:

comp. Benedicta, laxativa,

\section{A NCHVSA.}

132. ATCIIVSA officinalis foliis lanceolatis: fpicis imbricatis fecundis. Flor.-Silec. 161. Sp. pl.191. Fl. dan. t. 572 .

Bugloflum anguftifolium majus. Baub. pin. 256 . Loc. Europx prata et ruderata. Peremis, frequens. pharm. BVGlOSSI Radix, Herba, Syrupus, Aqua,

- Flores, Conferva.

QVAt. mucilaginofa, oleracea.

Superfuna.

vis: cordialis? exhilarans?

vsus: Melancholia? Hypochondriafis?

cunp. Fiores IV cordiales. Aque IV cordiales.

133. ANCHVSA imatoria tomentofa, foliis lanceolatis ohtulis, ftaminibus corolla brevioribus. Sp. pl. 192. Anchufa puniceis floribus. Butb. pin. 255 .

Loc. languedocia. Peremis, peregrina. IHARM. 
PHARM: ALCANNAE (SPVRIAE) Radix. WAi. rubia.

Vfitata.

vis: tinctoria.

VeYs:

conp. Tinture rubre. Figu. rubr. potabile... Spirit. Sal. arm. rubr.

\section{CYNOGI.OSS VM.}

I34. CYNOGLOSSVM affinale Raminibus corolla brevioribus, foliis lato-lanceolatis tomentofis fellilibus. Fr. Juec. т52. Sp. pl. 192.

Cynoglolim majus vulgare. Baub. pin. 25?. roc. Europre ruderata. Bionis, vulgatifima. pHAR. CYNOGLOSSAE Radix, Herba.

Qrat. mucilaginola, graveolens.

ris: aditringens, fubnarcotica, phthiriaca.

Dulia. vsvs: 'Tulfis, Coryza.

comp. Pilule Cynogloflix gr. V.

\section{PVLMONARIA.}

I35. PVLMONARIA officinalis foliis radicalibus ovato-cordatis cabris. Il. Jubc.163. Sp.pl. 194. Fl. dan. t. 483 .

Symphytum maculofun f. Pulmonaria latifolia. Baub. pm. 2.59 .

Ioc Eumpar rabies montium. Perennis, vulgaris. pHAR. JMONARIAL MACVLOSAE Herba. QVAL. mucilaginofa, oleracea. Dubia, Juperfuna. vis: glutinans, vulneraria, becchica.

virs: Hamoptylis?

comp.

SYM.

8;3. [Alcanne loco etiam adhiberi potelt Anchufec tutece rad. guam Urofina echioïtes prabet.]

135. P'ulmanaria non maculofo fulio C. B. cam maculoria convenit Specie et viribus, Licct maculis deflituatur. Fl. fues. 


\section{SYMPHYT VM.}

136. SYMPHYTVM officinale foliis ovato-lanceolatis decurrentibus. Hort. cliff. 47. Fl. Juec. 156. Sp.pl.195. Fl. dan. t. $66_{4}$.

Symphytum Confolida major. Banb. pin.259.

zoc. Europa auftralior.

PHARM. CONSOLIDAE MAJORIS Radix,

Conferon, Herba, Flores.

QVAl. mucilaginofa. Vjitata, Hb. Fl. fuperfua. vis: invifcans, glutinans, vulneraria, adflringens. vsvs: Hxmoptyfis! Diarrhœa, Dyfenteria, Hamaturia, Stranguria.

comp. Syrupus fymphyti, Empl. fractur. Empl.ruptur; Empl. opodelt.

\section{B O R A G O}

137. BOR A G officinalis foliis omnibus alternis, calycibus patentibus. Hort. ups. 34. Sp. pl. 197. Bugloflum latifolium, Borrago. Baub. pin. 236. ıoc. Normannia.

Annuch, cicur. PHARM. BOR R A GINIS Herba; Syrupus, $\nabla$, Flores, Conferva.

QVAL. oleracea.

vis: cordialis? exhilarans?

Dubia, fuperfina. vsvs: Melancholia?

comp. Flores IV cordiales. Aque IV cordiales.

\section{PRIMVLA.}

138. PRIMVLA veris: officinalis foliis denticulatis rugofis, limbo corollarm concavo. Fl. Juec. $17 \mathrm{r}$. Sp. pl. 204. Fl. dan. t. 433 .

Verbafculum pratenfe odoratum. Baub. pin.241.

I.oc. Europx prata. Perennis, vulgatifima: PHARM. PRIMVLAE VERIS Radix, Herba, Flores (Corollx), Conjerva, $\nabla$. 
Cral. fragrans, oleracea. Frequens, Hb. Superfun. vis: narcutica, exhilarans, anodyna, mervina. $R d$.

Aerrutatoria.

vors: Vertigo Flor. Paralyfis? Epilepla? Cephalaigia, Odontalgia $R d$.

COAR.

\section{CYCLAMEN.}

59. CYCLAMEN europeum corolla retroflexa. Hort. ups. 47. Sp. pl. 207. Facq. auflr.t.401.

Cyclamen hederx folio. Baub. pin. $30 \%$. Cam. epit. 35 . Loc. Pannonix, Auftrix umbrola nemorofa.

Perenne, manfuetum. PHARM. CYCLAMINIS Radix 3 j.

QYAL. mucilaginola, tum acris, fervida, (affata efculenta,) amaricans. Rarior.

vis: purgans, drattica, emmenagoga. Externe re: folvens, anthelminthica.

reves: Scirrhus.

comp. Vngu. Arthanitx.

\section{MENYANTHES.}

440. MENYANTHES trifoliata foliis ternatis. Fls fuec. 173. Sp.pl. 208. Fi. dan. t. 54 i.

Trifolium paluftre. Baib. pin. 327 .

1.oc. Europe paludes. Perennis, vulgaris. pHAR. TRIFUI,II AQVATICI Herba, Conferva, liflentia, $\Omega, \widetilde{V}$.

Qvas. amarifima.

Preftans.

vis: balfamica, tonica, faponacea, diuretica, anthelminthica.

vess: Cachexid, Scorbutus, Hypochondriafis, Arthriti, Nephritis, Rheumatifuns, Cacoëthes. cons. 


\section{Y SIM A CHIA.}

141. LYSIMACHLA Numularia foliis fubcordatis, floribus folitariis, caule repente. Fl. fuec. 177. Sp. pl. 211. F\% dan. t. 493 .

Numularia lutea major. Baub. pin.jog.

loc. Buropa aufralior. Perentes, cicur. PHARM. NVMV MARIAE Herba.

Qvas. fubacida, fubacris.

vis: laponacea, lubadfringens, vulneraria.

vsrs: Menorrhagia, Leucurrhœa, Vulnera interna. cosit.

\section{ANAGALLIS.}

142. $\mathrm{NAGALIIS} \mathrm{arven/is} \mathrm{foliis} \mathrm{ovatis,} \mathrm{caule} \mathrm{pro-}$ cumbente. Fl. fuec. 178. Sp. pl. 21. F\%. din.t. 88. Anagallis phoniceo. Hore. Baub. pin. 252. 1.oc. Europx arva. Amma, vulgaris. рнака. $\Lambda$ NAGLLIDIS Herba, $\nabla,\left(0 ; \Omega_{4}\right.$ Syr. Eelfentia.

Q NAL acris, inodora.

Rarior.

YIS:

vsve: Melancholia, Hydrophobia.

Cons.

\section{SPIGEI,IA.}

143. S P I GELI A Anthelmia caule herbaceo, foliis fummis quaternis. Syft.nat.13.p.166. Amen. acad. 5. p. 133. t. 2. * Sp pl. 213.

Spigelia quadrifolia, fpicis terminalibus. Brown. jam, $156 . t .37 \cdot f \cdot 3$.

coc. Brafilia, feritur in Jamaica. Annua, fera. rHARM. SPIGELIAE Herba.

QVAc. tetra, fortida.

Rurior.

vis: narcotica, expellens, anthelminthica!

VSVS:

143. [SPIGELIAE Her.man e Spigelia marylandica Limn inant. sjS. defumi, Cel. Bergizss mat. med.64. mavult, utpote qux Antheimiam viribus finerare fertur.] 
TSrs: Lumbrici!

COMP.

\section{OPHIORREIZA.}

14. OPHIORKHIZA Mungos foliis lanceolato - Cari.

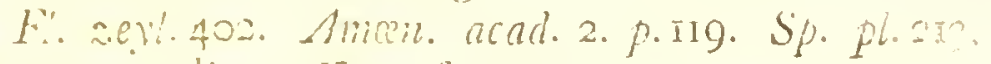

Mingos radix. Kempf. amcen. 577.

Ioc. Ina Zeylona, Sumatra. Perennis, peregrina. PHARM. SERPENTYM Radix.

orst. amariino.

vis: expellens.

rsss: Mifrlara venenata, Hydrophobia, Rabies,

Srrochiss.

contr. Lapis de Goa.

\section{PLVMPAGO.}

145. PLVMBAGO emropen folis amplexicaulibus lancculatis leabris. Fort. cliff.53. Sp.pl.215.

I epidiun dentillaria dictum. Baub. pin. 97.

Luc. Neapolis, Syracula, Gallia autralis.

Perennis, manfueta. PHAM. DENTARIAE RadiX.

Qy.i. acris, feptica. Exoleta, prefans.

vis: ianfica.

vis: Gdontalgia.

coist.

\section{CONVOI. VVITS.}

146. CONVOLVVLIS Srammonic folies fagittatis poftice truncatis, pedunculis teretibus fubtrito. ris. Sp. pl. 213.

Convolvulus Tyriacus. Morif. bift.2.p. 22.f. r. t.3.f.5. toc. Syria, Myfia, Cappadocia per Smymam. Peremis, peregrina. PHARM. SCAMMONIVM Crudum Cir. vj. Dian crodium $\theta$ 月. Refina $\theta$. 
Qy.A. acri-amaricans, naderofa, gummolo-relinoia.

linit?, criniz

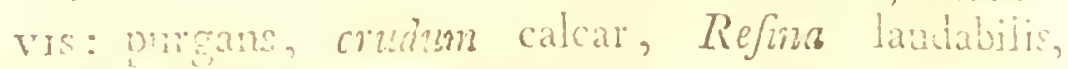
Dixis ne. epsimus.

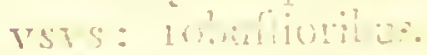

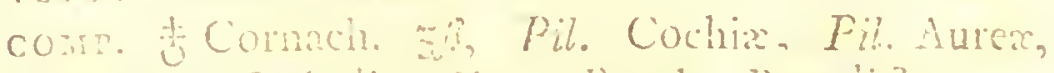

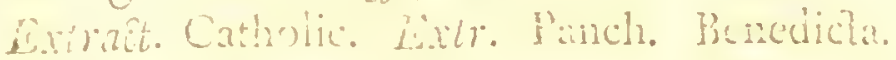

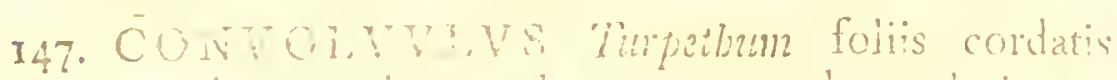
arovlatis, avie mumbraraco-quadranguiari, pe-

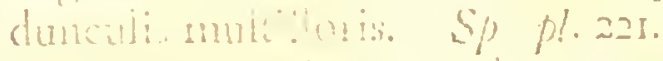

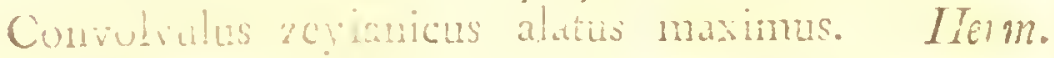
$\approx e^{2 !} \cdot 177 \cdot t \cdot 1-9$.

loc. Leylona, Malabalia. Peremeis, perentina. pHAM. "IVR H THI Radix 5/. Eutraitum.

QAL. gummora, porola, bilocularis, lubinfipidoactis, mavloufa.

vis: purgas tardirfoula.

vsvs: Altritis, Mrdrops, Chronici.

comp. Extr. pancism. Pil. cochix, Pil. Agarici, of Avthotic.

148. CONVOI, VVR.YS galapa folis difformibus: cortatis angulatis cbloncis Janceolatisque, caule volubili, pedunculis unitoris. Mantiff. pl. 43. Convolvulus americanus Jalapiun dictus. Raj. bift. 724. roc. America.

IHARM. JALAPPAE Radix 5 $\beta$. Extractum $\partial j$. Refma $\theta \Omega$.

QVAI.

I48. J A L A P P A E rad. acutinimus Bolanicus Plamier, qui ter

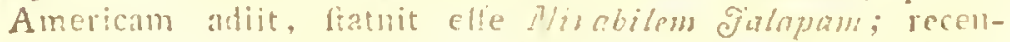

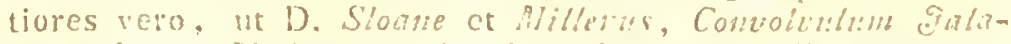
gan. bmono Mlillerus, qui radices Jalape per Hon:lboum ex Anerica reportavit, et l'harmaconolis Londinemfitus vendidit, habuit ratices Convolvuli hujus, quæ ommibas numeris vera ollin in aii relpondebant. Whif, in mat. med. o. [ Cel.

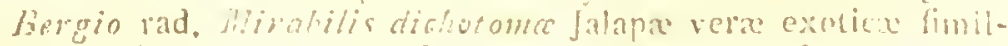
lima videtur; Minbijis Inlapa et Mlirab. Longiflobe radices non purgare lacm expertus eft. Mat. Miat. Go.] 
orat. nauleola, acris, odore fapore dof facillima. Trita, beroica.

VIS: purgans. Refina calcar purgantis.

vors: frigitis, licculentis, infantibus. Vermes.

conp. Spec. diajaliappe.

149. CONTOLVYLTS Mecbaacanna [caule vo. lubili, foliis cordatis, fructibus nuarimis tomen: tolis. Berg. mat. 63.]

Jeticucu. Nhircy braf. 4 .

Loc. America meridionalis: Brafilia, Ouito.

Peremis, peregrina. PHARM. MFCHOACANNAE Radix z/s. Extraimm. exar. fusinipido-xcris, inodora. Trita, eximia. vis: eccoprotica, fecuilima, roboraiss.

ISTs: infantibus, Oclema.

Cuis.

150. CONVORVLITS Soldanella folis reniformibus,

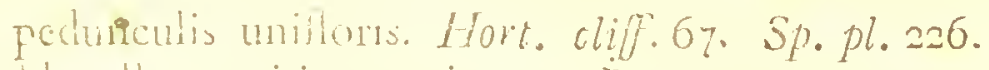
Soldanctla maritina misor. Baab. pin. 295 .

ive. Anglix, Frifia naritina. Peremis, manfueta: YHARM. SOLD MNEILAR. Herba zj. QVis. naulcofa, aci is.

vis: draltica, hydragoga.

visus: Hydrops.

cusis.

Exoleta, infida.

\section{CINCHONA.}

I5r. CINCYIONA affeinalis panicula brachiata. Sp. pl. 244. Syjt. nat. 13. p.178. *

$$
\text { Ji: } 2
$$

Quirl-

149. [ MTechoncannam quamdam Conzolvatuts pranduratus quoque largitur; an frecte ab ifta quse in libalila colligitur,

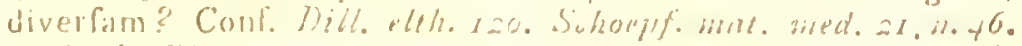

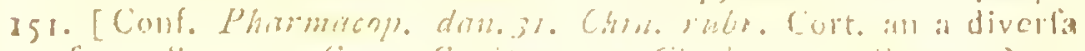

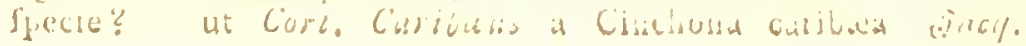


Quinquina. Condam. Act. parif. 1738. p.226. t. 5. fig. A-E. tab. 6. fig. F. - S. Geoffr. mat. med. 2. p. iso. roc. Loxa Peruvia.

Arbor, peregrina. pHarm. CHINAE Cortex 3j. Fefentia, Exirnactum. QVAL. tenaciflime amara, carncs amaritie inficiens. Tivita, beroica. ris: tonica, adfringens, roborans, fublaxans, antis feptica.

rovs: Febres, Anorexia, Calculus, Cedema, Cachexia, Gangrana, Hylteria. [Hxmorrhagix, Variolix, Phthifis, 'Tuflis convulf. Scrophulix.] COMP.

\section{PSYCHOTRIA.}

152. PSYCHOTRIA emetica herbacea procumbens, foliis lanceolatis glabris, Ripulis extrafoliaccic fubulatis, capitulis axillaribus 'pedunculatis paucilloris. Suppl. pl. 144.

Ipecacuanlia. Pif. brajul. 101? Marcyr. braf. 17? zoc. Regni Mexicani provincia Gironenfis. Perennis. PHARM. I PECACVANHAE VVLGARIS Radix?

QVAI.

Cort. S. Lacio a Cinchona cymola etc Kentifi exp. and obf. on aniw fpecies of betk. Lond. I784. Cf. Blumenb. med. bibl. $2 . p .485$.

352. Ex teftimonio Rhizotomi cujusdam Mexicani, qui Ipecacnanham venalem frepius collegerat, et certo fe noviffe afteverabat, haec vera firps eft, cul radicem Ipecacuanba venalem debernus. Suzpl. pl. l. s. Sy/t. veg. 14. p.214. [Conf. Jpecacuanlua brafilienfis radice cmetica. Jetiv gaz. t.59. f.g. Berg. mat. med. 70 . et infia Viola Ipecacuantha. Secundum Phormacop. ranicum $p$. st. Ipecacuanha in genere pro Er:phorbic Tpencuanhe radice habenda eft. Alia Ipecacuanha Grianenfim elt Borhanvia diandra Aubl. guan. 4. alia Infularum Caribearum, Aliclepias curaffavica, Oldend. Caril.. 92. alia Ca:olinenfium, Podophyllum peltatum, alia Virginiano. rum, Spirca trifoliata, et Pfeudipecacmanna virginica, Triofeam perfoliatum. Schoepf. mat. med, amer. p. 86. So. et 23.] 
Qrad. mafeola, amaricans, et leniter pungens. Trita, eximia. vis: emetica! fudorifera, corroborans, adfringens. vsus: Febres Criticat, Intermittentes et Exacerbantes? Leucorhoca, Hemorrhagia uteri, Afthma, Tullis, Pleuritis, Peripneumonia, Diarrhœa, Dyfenteria! Vermes. Aman. acad. 8. p.245.

\section{COFEEA.}

153. COFFEA arabica Horibus quinquefidis difpermis. Aman. acad. 6. p. 160. t.160. Sp.pl.245. Jafminum arabicum, lauri folio. Fuff. at. ris. p. 388 . t. . .

L. oc. Arabia felix.

PHARM. COFFE Semina, uftulata. CVAL. ufta empyreumatica.

Arbor, feris.

vis: exficcans, excitans, calefaciens, pellens, carminativa, diuretica, antivenerea, anthelminthica. vsvs: Polyfarcia, Sopor, Cardialgia, Cephalalgia, Calculus. Nocet Iypochondriacis, Hyttcricis, Melancholicis, oculis,

CO:is.

\section{LONICERA:}

154. LONICER A Periclymenum capitulis ovatis imbricatis terminalibus, foliis omnibus diftinctis. Hort. cliff. 58. Iil. ficec. 139. Sp. pl.247.

Periclymenum non perfoliatum germanicum. Bakb. pin. 302.

roc. Eumpa auftralior. Arbufcula, cicur. PHARM. CAPRILOLIL Stipites, Herba, Aque. (ख)A. ingtata. Dubia, exaleta. 
vis: vulneraria, mundificans.

vsvs: Ophthalmia $\nabla$ ? Gargarisma Herbx? COMP.

I55. L. ONICER A Symphoricarpos capitulis lateralibus pedunculatis, foliis petiolatis. Sp.pl.249.

symphoricarpos foliis alatis. Dill. cltb. 3r7. $t .2$ 2 8 . $f \cdot 360$.

t.oc. Virginia, Carolina. Frutex, cicur. -harm. SYMPHORICARPI Stipites.

QVAL. Afyptica.

Inil.jitata.

ris : fubadiftingens.

vsrs: Febres iintemittentes!

contP.

556. I.ONICERA Diervilla raccmis terminalibus, foliis ferratis. Sp. pl. 249.

Diervilla. Hort. cliff. 63. t.7.

I.OC. Canada.

Frutex, manfuetus.

PHARM. DIER VILL AE Stipites.

QVAI. maufeofa.

vis: pellens.

vsvs: Dyfuria, Gonorrhœa, Syphilis!

COMP.

\section{CORIS.}

157. CORIS monfpelienfis. Hort. cliff. 68. Hort. ups. 46. Sp. pl. 252 .

Coris crerulea maritina. 'Baub. pin. 280."

цoc. Gallia Narbonenfis, Creta. Bicnnis, manfueta. phapm. COREOS Herba, Decoetum.

QVAL. amarifima, naufeofa. Eximia, inufitata. vis: pellens.

vove: Syphilis.

COMI. 


\section{Y}

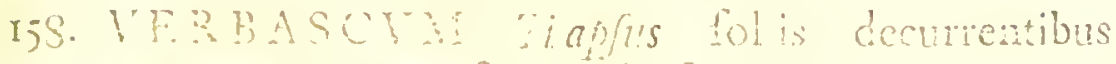

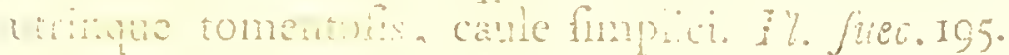

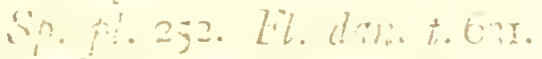

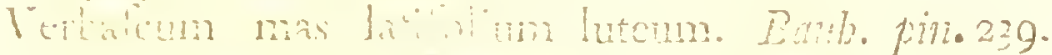

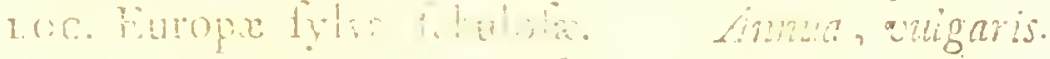

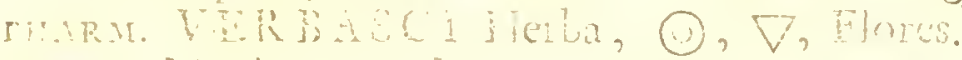

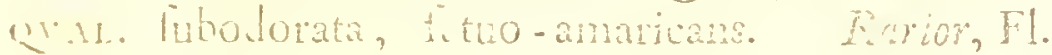
fuperfain.

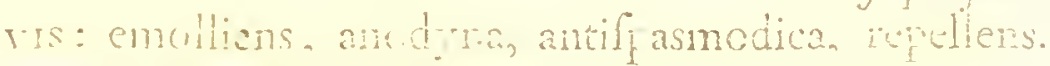

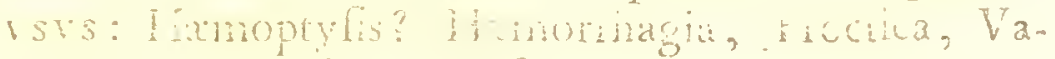
rix ani, 'Tullis. Fithifs,

COMP.

I59. TERPASCYMI minm folis cond oblongis putivlatis. Fl. frec. Is,6. S\%.pl.253.

Vebaloun nigrum, lore ex lutco purerafcente. linth. 1)in. 240 .

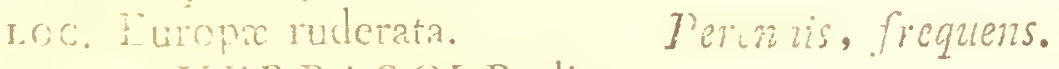
PrAR TIRRASCI Radix.

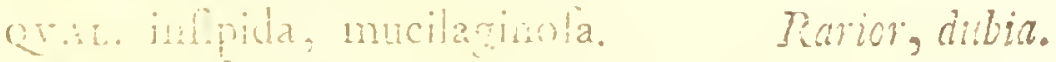
ris: finctacien, emolicis.

vers: Puronicha.

Co:Tip.

\section{B) TVR $\mathrm{T}$}

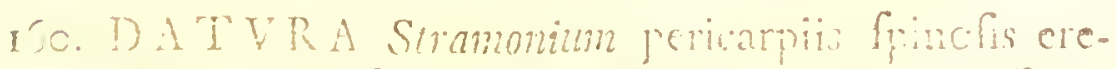

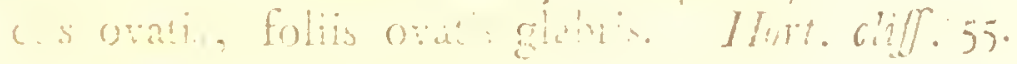

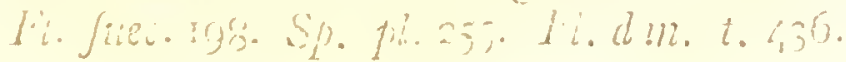

I: Sola-

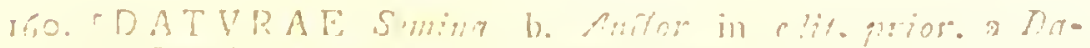

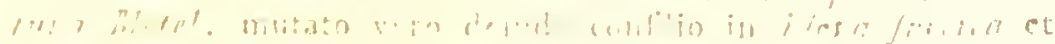

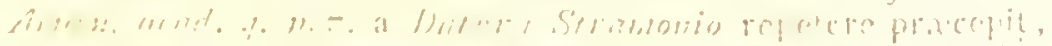

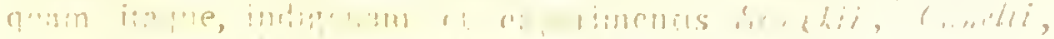

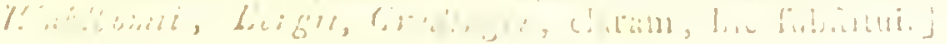


Solanum fatidan pomo finofo oblungo, flore .ibo. Ban. pin. 163 .

wos. Furopx auflialionis ruterata. Anmun, cicur. gYARA. "YRAMONII Herba, Extraimm.

D) ATRAE Semina.

Qytr. amara, viroli, torica. Sem. infipida. Cnute. VIs: iumbutica, amnentica, phantaflica, demestans, exhilatins, anodyna, hyphotica, paregorica, repcllens.

vsvs: Cumvulfones, Amentia, Mania, Epilepria,

Carcinona, Varix ani, Combuluna.

conp. Collopef. phantaficial Bartbol.

\section{HYOSCYAMVS.}

16r. HYOSCYAMVS nizer folis amplexicaulibus finuatis, thoribus fefilibus. Fl. fuec. 199. Sp. 1l. 257. Hyofeyamns vulgaris et niger. Baub. pin. 169 .

zoc. Eurnpx ruderata. Bicmis f. amma vulyatifima. pharm. HYOSCYMML Radix, Lerba, Semma, (D). QAL. fubinfipida, viroli, toxica. Farior, caute. vis: narcotica, phantaftica, dementans, anodyna, hypnotica, antifpalmodica, repellens, refolvens. vsvis: [Paralyfis, Convulfiones, Mánia, Epilepha. Tuflis, Hamoptyfis, Olontalgia (funus), Varix ani.

Eomp. Philonium, Pil. cynoglofix, Trocb. alkekengi, Vhagz. populieum:

\section{NICOTIANA.}

162. NKOTIANA Tabicum folitis lanceolato - ovatis Chlilibus decurrent hus, horibus acuris. Sp.pl. 259 .

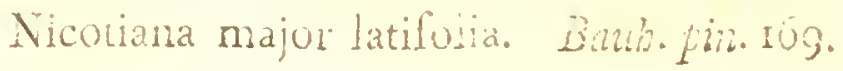

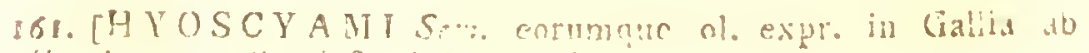

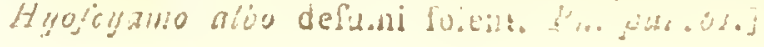


Loc. America.

Anmua, manfueta. PHAR.' TABACl Herba, Pulvis, oo, Exiraitum gr. ij. Syrupus siij. Semina.

o. A r naleota, virola, toxica, acris. Trita, preftans. in: anolyna, antilualmodica, narcotica, phantaftica, paregorica, repellens, errhina, exanthematica, phthiriaca, refolvens, vulneraria.

¥sis: Vicus, Scabies, Tinea, Ocdema, Tuflis, 'Pertiana, Plentis, Obfipatio alvi, Syr. Alluma, Coilca, Hylleria, Djienteria, ICtcrus, Scirrhus, Hidrops.

comp. Finglt. Nicotiante.

\section{ATROPA.}

153. A TR OPA Mandragora acaulis, fcapis unifloris. Sp.pl.259.

Mandragora fructu rotundo. Baub. tin.169. Loc. Creta, Cyclades, HiSania, Italia, Ruffia temperata.

Poreminis, manfueta. PHARM. MANDRAGORAE Radix, Extraitum, (O). Qyal. virofa, toxica. Cort radicis acris. Exoleta, cante. vis: narcotica, phantafica, dementans, anodyna, antīpamodica. Cort. rallicis purgans, pellens, abortiva.

vsss: Hodagra, Tumores feirrhofi, Epilepfia, $\mathrm{Hy}$ Alerica, Colica.

conis.

16. A TR คPA Bellacionn caule herbaceo, foliis ovatis intection. Sp. pl. 260. Fl. dan. t.758. Facq. . antli. t. 303.

Solanm maniacum multis. Baub. biff.3. p.6rr. wor. (jemmia Aigin. Percmis, cicur.

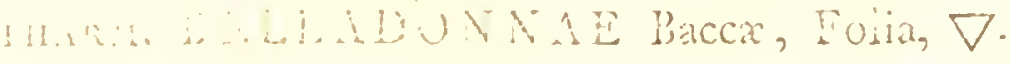
$\therefore 5$ a cas. 
eyal. fubinfipida, virofa, toxica. Imfarata, pretvis: narcotica, phantafica, paralytica, anchlyna. - antifipasmodica, repellicus.

vsve: Dyenteria, Luberto, Cacoüthes, Bubo man. narum, Carcinnma! Fiftula, Prolaplus ani. [Convuliones, Epilepria.]

coms.

\section{PHYSAT.IS.}

165. PIIYSAI.IS Alkekengi folits geminis integris acutis, caule herbace inferne fubramolo. Hort: ups.49. Sp. pl.z62.

Solanum veficarium. Bauh. pin. I66.

Ior. Europar aufralionis forobes. Peremis, cicur. rharm. ALKEKENGI Horba, Bacca, $\nabla$, Semina. cr.m. acidula, anaricans. HB. Sem. fuperflua. vis: fubanodyna, diuretica, refrigcrans, eccoprotica.

vsve: Mrthritis, Dyfuria, Nephritis, Phlogofis, Hydrops.

cosis.

\section{SOLANVM.}

366. SOL $\triangle N Y M$ Dulcamara caule inermi frutefente Hexuofo, folis fuperionibus hatatis, racemis cymois. it. fuec. zor. Sp.pl.264. Amen. acad. 8.p.63. Fl. dan. t. 60\%.

Solanum feandens feu Dulcamara. Baub. pin. I6?. soc. Europx fepes humitiulculx. Frutex, frequens. FHAR.1. DVI.CAMARAE Stipites sij-iv.

Qva1. fubdulcis, amara, nadeola. Rorior, beroica. vis: andyne, repclicus, pellens, puriticans, diuretica. 
rops: Rheumatimus, Mithritis, Pleuritis, Icterus, Athma, Scorbutus, Dysmenorrhar, Dyslochia, Contulura, Scabies, Syphilis.

rosip. Decoita mundificantia, Influf. traumaticum Fitll.

I67. SOLANVM nigrm caule inemi herbaceo, foliis ovatis dentato-angulatis, racemis diftichis nutantibus. Fl. fuec. 200. Sp.pl.266. Fl. dan.t. 460. Solanum officinarum. Batib.pin. $\mathbf{6 6}$.

coc. Europe ruderata. Ammu, vulgatifina. рніR.r. SOLANI Herba, $\nabla$, (O).

CraL. virofa. Rarior. vis: anodyna, hypnotica, paregorica, repellens, reficerans.

vsrs: Phlegmone, Proctica, Scirthus, Paronychia, ricus.

comp. Emplaft. de Solano.

\section{CAPSI C.V M.}

163. CAPSICVM ammum caule herbaceo, pedunculis folitariis. Sp.pll.270.

Piper indicum vulgatiflinum. Baub. fin. roz.

coc. Brafilia, Mexico, Barbados. Ammin, fubmaneta. PH.R.M. PIPERIS INDICI Fructus. [Semina.] pis. acris, feptica. Culinaris Indis, eximia, ravior. vis: rubefaciens, fimulans, tufiva.

rsva: Afthra, Coryza, Anorexia. [Febres intermittentes.]

COMP.

\section{STRYCHNOS.}

rog. STRYCHNOS Nibx romica foliis ovatis, cauJo incrui. Fl. zeyl. 91. Sp.pl.271.

[Serych- 
[Strychnos folis ovatis obtufis quinquenervils: nervis evanefcentibus. Berg. mat. 88. ]

Caniram. Rheel. nial. 3. p.67. t.37.

roc. Malabaria, Zeylona.

pHARM. VOMICAli Nux, $3 \beta$.

QVAL. amara, virola.

Arbor, peregrina. $v i s:$ cmetica, pellens, anthelminthica.

vsve: Colica, Cardialgia, Gonorrhea, Febres int termittentes, Txuia, [Morfura ferpent.] cons.

170. STRYCHNOS colubrina foliis ovatis acutis, cirrhis fimplicibus, $S p . j l .37 \mathrm{I}$.

[Strychnos foliis conjugatis ovatis trinerviis, cirrhis fimplicibus. Berg. mat. 90.]

Modira-caniram, Rused. mal. 8. p.47.t.24.

r.oc. Malabaria, Timor, Solor. Arbor, peregrina. pHarir. COLVBRINI VVLGARIS Lignum (radicis) zs.

orar. fupecta, acris, amarifima. Exoleta, cauta. ris: cmetica, pellens, fudorifera, anthelminthica, fatuans.

vis: Murfura vencinata, Quartana. COMP.

\section{GNATIA.}

171. IGNA T IA anara. Suppl. pl. 149.'

[Strychos Ignatii foliis ovatis acutis quinquenerviis: nervis continuatis, fructibus maximis. Berg. mat. 89.]

Cucur-

170. Hanc a Str. Nirse vomica non diverfam effe, Botanici in India degentes contendunt.

bigua colubrina vulgo appellantur apud Indos omnia illa Iigna, quate aquan in pocula ex is tornata infulam pura anarsie impregenant et auridota putantur. Hinc in oficinis pharmacenticis lighin diverla nomine Ligni colngrini inveniuntur, interdim moris, interdum minoris efficacie.

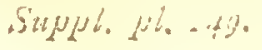


Cucurbitifera malabathri folio, fandens, Catolonsay et Contarí Philippinis orienialibus dicta. Pluk. mant. 60.

r.oc. India orientalis.

peregrina.

FHARM. I GNAT II Faba.

CYAL. amarifima.

Rarior, beroica.

Vis: tonica, narcotica, pellens, diuretica, fudori-

fera, emmenagoga, anthelminthica.

rsis: Morbi ventriculi, Jebres Exacerbantes, In-

teimittentes, Morbi evacuatorii ab Atonia, Soporofi, Afthma, Ecclamplia.

COMP.

\section{CORDIA.}

172. CORDIA Myxa foliis ovatis fupra glabris, corymbis lateralibus, calycibus decemflriatis. $S p$. pl. 275 .

Sebeften officinarum. Dill. elth. 340, t.255. f. 3 j.

Ioc. Aegyptus, Malabaria.

Arbor, fera.

PHarit. SEBESTEN (Drepa).

QYA.. mucilaginola, dukis.

rs: nutriens, invilcans, lubricans, humechans, ec-

coprotica, adfringens.

rsvs: Raucedo, Tullis, Stranguria.

cosis.

\section{RHAMNVS.}

173. RHAMNVS cationticus fpinis terminalibus, flo. ribus quadrifidis diojcis, foliis ovatis, caule erecto. Fi. Juec.202. Sp. ph. 279 .

Rhammus catharticus. Baub. pin. 478.

roc. Europe faltus. Arbor, vulisaris. PHARM. SPINAE CERVINAE Pacco sij, Succus viritis.

QYs. amita, lutea. 
vis: purgans.

vsve: Cachexia, Arthritis, Hydrops, Syplilis. comp. Syrupus domellicus ३う.

174. RHA MNVS Frangilla inermis, foribus monogynis hermaphroditis, foliis integerrimis. $F l$. juec. 203. Sp. pl. 2\$o. Il. dan. t. 278.

Alnus nigra baccifera. Baub. pin. 428.

roc. Europa humidiufeula. sibufcula, vulgaris. pHARм. FRANGVL A E Cortex, infusim $\frac{5}{3} / 3$.

QVAL. amaricans, hutea.

Rarior, beroica.

vis : purgans.

vsvs: Hydrops, Scabies, Althma humidum, Va. rix ani.

co.tre

375. RHAMNVS Zixyphus aculeis geminatis: altero recurvo, floribus digynis, foliis ovato-oblongis. Hort. cliff. 96. Sp. pl. 282.

Zizyphus. Dod. pempt. 807.

coc. Italia, Verona. Arbor, manfueta, ferilis. PHARM. JVJVBAE Baccx, Syrupus.

CYAL. dulcis, mucilaginola.

vis: invifcans, lubricans, edulcorans, expectorans. vsvs: Tullis, Stranguria.

comp. Loboc fanum, Syrupus de jujub.

\section{CEANOTHVS.}

176. CEANOTHVS anericanus folis trinerviis. $S p$. pl. 284. Mill. ic. t.86. Dubam. art. 1. p.138.t.51. Evonymus novi belgii, coni feminx foliis. Contmel. bort. 1. p.167. t.86. Raj. dendr. 69. xoc. Virginia, Carolina. Frutex, manfuetus. PHAR AE. CEANOTHI Stipites. NAL. rubra.

Rara. 


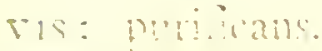

vi: : sifilitis!

CUis?.

\section{RIBES.}

I7?. RIBES rubrum incrme, racemis glabris pendulis, Horibus planiuleulis. 1\%. fiec. 205. Sp. pl. 290. Groflularia multiplici acino i. non fpitolit hortenfis rubra, five Ribes officinarum. Baub. pin. 455. Loc. Europa, priertin Weftrobotnia. Frutex, cicur. PIARM. RIBIVM RVBRORVM Bacce condite, Rob.

erit. acida, rubra. Culinaris, trita, preftans. is: refrigerans.

vsts: Febres Phlogillici, Putride, Sitis, Aelus. conp.

I7.9. R I PTHS mignum ineme, racemis pilofis, floribus oblongis. I\%. huec. 207. Sp. pl. 20r. Fl. dan. t. 556 .

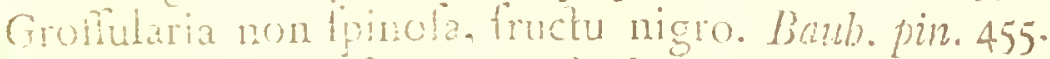
Loc. Europa praterin Weftrobotnia. Fratex, cicur. FHA.: RIBI!M. XIGRORVM Herba, Bac.

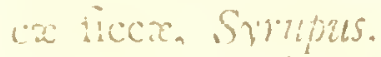

arde acidiucula. Herb. tetra. Rarior, treflans. is: anodrna, repellens, pellens, diuretica, purifcans. [Brecr. refriereans.]

ros: Ancina! Fibes exanthematicx, Rabies, Hydrophobia, Dylenteria! Mortura Eerpentun. [Rhcumatifmus.]

$\operatorname{cosin}$.

\section{HEDER $\Lambda$.}

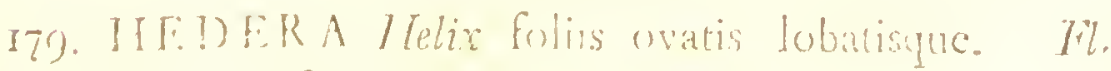

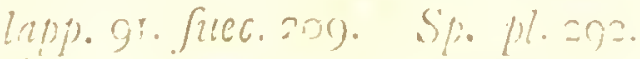

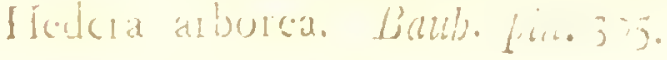


LOC. Europa auftralior.

Frutex, cicur. PHARM. HEDERAE ARBOREAE Lignum, Folia,

Refina, Bacca.

QVAl. Fol. amara. Bace. acila. Rarior, preftans. vis: vulneraria, Cophinicans.

vsvs: Vlecra, Fonticuli, Atıophia, Ráchitis, Ozæna, Epiphora.

cosp. Vingu. althxx.

\section{VIT I S.}

180. VITIS viniferc folis lobatis finuatis nudis. Sp. pl. 293. Scbinidel. ic. I. p.32. t.7.

Vitis vinifura. Baub. pin. 209 .

roc. Orbis totus temperatus. Fritex, fubmanfuetus. PHARM. VI'TIS Folia.

AGRESTAE Symprs.

CORINTHIACAE, pulpa, loboc. VINVM album; rinenanum, rubrum.

Spiritus, Acetum, Acettum deffillatum: Qral. Fol. acidula, fubityptica. Pafinl. acidulo: dulcis.

Irita, eximia.

vis: Fol. Subadhringens. Fafin. nutriens, lubricans, fublaxans.

rsve: Tuflis, Stranguria.

comp. Syrup. Althxix. "rr. Ervimi? Syr. hyfopi. Conf. hamech. Pallule Lavitiva.

\section{LAGOECIA}

I8I. L A GOFCIA Caminoides. Hort. cliff. 73. ups.53. Sp.pl. 204 .

Cuminum fylveftre, capitulis globofs. Baub. pim. 176 . soc. Creta, Lem us, Galatia. Ammit manrereta. PHARM. AMMIOS VETERVM Ferba, Semina. 
CVAL. aromatica.

Preflans, exoleta.

ris: Aomachica.

vsis: Anorexia.

COMP.

\section{ALLA M A NDA.}

182. ALLAMANDA catbartica. Mantiff. 214. Sy/t. mat.13. p. 209.

[Orelia grandiflora. Aublet. guian. 27. tab. 106. Chryfolpermum granditiorum. Roiander. Rottböll act. bafn. 1778 . 5.288 .]

Loc. Guianx ripx et.litora. Frutex, fsandens, peregrinus. PHARMr. ALLAMANDAE Folia, Infiufitizl.

QVAL. lactefcens, amara.

Inufitata.

vis: cathartica, tonica.

vsvs: Colica piclonum!

comp.

\section{VINCA.}

IS3. VINCA minor caulibus procumbentibus, foliis lanceolato-ovatis, Hioribus pedunculatis. Hort. cliff: 77. Hort up. 53. Sp. pl. 304.

Clematis daphnoides minor. Buth. pin. $30 \mathrm{r}$.

loc. Germania, Europa auftralior. Peremis, cicur. PHAR II. VINCAE PERVINCAE Herba.

(Nat. amara, inodora. Rarior, cauta. VIS: viridis pellens, ficc. adftringens, iophifticans. vsus: Jaxitas.

Conp. Gargarifmata.

\section{NER I V M.}

184. NERIVM antidyfenterium, foliis ovatis acuminatis petiulatis, Fir. zeyl.107. Sp. pl. zo6.

Nerium indicum, fili juis anguttis erectis longiflimis. Burm. zejl. $167 . t 77$.

noc. Malabaria, ieylona. Friltex, peregrmis. 
PHARM. CONESSI f. PROFLVVII Cortex. QVAL. lactefcens, acris. vis: anthelininthica.

Ignota.

vsvs: Diarrhœa, Dyfenteria, Odontalgia.

COMP.

\section{PL V MER I A.}

185. PLVMERIA alba foliis lanceolatis revolutis, pedunculis fuperne tuberofis. Sp.pl.zo6. Facq. bift. 36. t. 174. f..I2.

Apocynum americanum frutelcens, longifimo folio, Hore albo odoratifino. Comm. bort.2. p. 47. t. 24 .

roc. Infulx Caribax. Arbor, peregrina. Pharm. PLV MER IAE ALBAERadix, Decoctum? QVAr. lactefcens.

vis: vulneraria.

vsve: Syphilis.

COMP.

\section{$D I G Y N I A$. A S C LEPIAS.}

186. A SCLEPIAS aftbmatica fruticofa volubilis hir. futa, foliis oppolitis petiolatis cordato-ovatis fupra glabris integerrimis, umbellis paucithoris. Suppl. pl. rizi.

Afclepias zeylanica, vincetoxici radice praflantiore. Burm zeyl.36. Flor. zeyl. 490.

Apocynum zeylanicum fandens, foliis laurinis. Herm. prod 4I3. Manughawal Zeyl.

цoc. Zeylonæ fylva. Frutex, peregrimus. rharm. ASClEP. ASTHM. Radix, Decoetum, Syrrupus.

Qval. fubdulcis, acriulcula, fubnaufeofa, odore Vincetoxici. Herm. Eximia. 
vis: incidens, expectorans, eccoprotica, Jubemetica. Herm.

rsvs: Tullis. Herm. Afthma ferolum. Limn.

887. ASCLEPIAS Vincetoxicum foliis ovatis bafi barbatis, caule erecto, umbellis proliferis. $F$. fuec. 212. Sp. pl.315. Fl. dan. t. 849.

Vincetoxicuin. Dod. pempt. $40 \%$.

Loc. Europx arida. perennis, frequens. PHAR.r. HIR V N DIN ARIIAERadix zj. Herba, $\nabla$. QVAl. lactefcens, fubvenenata. Trita, dubia. Hb. fuvis: pellens, diuretica, fudorifera, emmenagoga, expellens.

vsvs: Hydrops! Variolæ.

comp. Ellent, alexipharm. Stahl.

\section{HER NIARIA.}

188. HERNIAR IA glabra glabra. Fl. fuec. 2r3. Sp. pl 317. Fl. dan. t. 529 .

Polygonum minus f. Millegrana major. Baub pin.28r. roc. Europx arida.

PHARM. HER NIAR I AE Herba.

QVAL. inodora, infipida.

Perennis, frequevis.

VIS: adfringens, diuretica?

- Exoleta, dubia.

v'sv: Scotomia, Ophthalmia? Hernia? Hydrops?

Calculus, Carcinoma.

CO.MP.

\section{CHENOPODIVM.}

189. CHENOPODIVM Bunus Hemicns foliis triangulari-fagittatis integerrimis, fpicis com: ofitis aphyllis axiliaribus. 1\%. Jutc. 214. Sp. p! 313. Fl. dian. t. 579 .

Lapathum unctuolum, fillo triangulo. Baub. pin. 115. Fa

I. OC. 
IOC. Europx ruderata.

Perennis, vulgaris. PHARM. BONI HENR ICI Herba.

QVAL. oleracea, fatua.

Rarior.

VIs: refrigerans, humectans, vulneraria, eccopro. tica.

vsvs: Paronychia, Oedema, Varix ani, Podagra, Pleuritis, Phthifis.

COMP.

190. CHENOPODIVM rubrum foliis cordato-triangularibus obtufufculis dentatis, racemis erectis compolitis fubloliofis caule brevioribus. Il. Juec. 217. Sp. pl. 318.

Atriplex fylveftris latifolia. Baub. pin. 119.

Loc. Europx ruderata. Anmua, pafim. PHARM. A T R IPLICIS S YLVES'TR IS Herba. QVAL. aquofa.

vis: dubia.

vsvs : incertus, fuibus?

coMP.

191. CHENOPODIVM Botrys foliis oblongis finuatis, racemis nudis multifidis. Hort. cliff. 84 . upf. 55. Sp. pl. 320.

Botrys ambrofioides vulgaris. Baub.pin. 138 .

I.ọc. Calabria, Hetruria, Narbona, [Rufia.]

Annua, cicur.

PHARM. BOTR YOS Herba, Semina.

eyal. fragrans, graviufcula. Rarior, preffans. VIs: fubinebrians, refolvens, pellens, carminativa, emmenagoga, anthelminthica, antifpafmodica. vsvs: Afthma, Orthopnoa, Phthifis, Tullis convulfiva.

COMP.

192. CHE-

191. [Chenopodium ambrofioidos frequentius adhiberi folet.] 
192. CHENOPODIVM anthelminthicnm foliis ovato - oblongis dentatis, racemis aphyllis. Sp.pl. 320.

[Scirepf. qiat. med. 3I.]

Chenopodium lycopi folio, perenne. Dill. elth.77. t. 66. f. 76 .

Loc. Penfylyania.

Pereniis. PHARM. CHENOP. ANT HEL M. Semina 弓ुj. CYAL. graveolenti - fragrans. Eximia, inufitata. vis: anthelminthica!

rsis: Lumbrici.

comp.

193. CHENOPODIVM Vulvarin foliis integerrimis rhomboideo-ovatis, fluibus conglomeratis axillaribus. Fl. fuec. 222. Sp. pl. 321.

Artiplex fatida. Baub. pin. II9.

zoc. Europx culta.

PHARM. VTLVARIAE Herba, Conferva.

QYAL. hircofa, urinaria. Inufitita, preftans.

vis: antilpasmodica.

vsus: Hyfteria.

comp. Elect. hyfteric. Fulleri.

\section{BETA.}

194. BET A vulgaris floribus congeftis. Syft. nat.r.. p. 195 .

Beta rubra vulgaris. Baub. pin. II8.

loc. Europx auftralioris maritima. Biennis, cicur. PHARM. P,ETAE R VBRAE Herba, Radix.

QTit. aquofa, dulcis fubaromatica. Culinaris, exoleta. vis: diluens, humectans, erthina. Rd. fucc. (Succiss emolliens?) Suppofitoria.

vsis: Ophthalmia, Coryza Sicca, Cephalalgia, Odontalgia.

COMP. 
195. BET A Cicla floribus ternis. Sy/t. nat. 12. p.95. Beta alba vel pallefcens, qux Cicla officinarum. Baub. pin. II8.

coc. Europa auftralioris maritima. Anmua, cicur. PHARm. BE TAE ALBAE Herba, Sucus. QVAL. pracedentis. vis: procedentis.

vsvs: pracedentis.

COMP.

\section{VLMVS.}

996. VLMVS campeftris foliis duplicato - ferratis : bafi inrqualibus. Fl. Juec. 226. Sp. pl. 327. Fl. don. t. 632 .

Vlmus campeftris et theophrafti. Baub. pin. 426. LOC. Europx pagi.

PHARM. VL Míl Cortex medius.

QVAL. flavelcens.

vis: adfringens, vulneraria.

vsvs: Alcites, [Vulnera, Combuftura, Scabies!] COMP.

\section{GENTIANA.}

197. GENT I A A lutea corollis lubquinquefidis rotatis verticillatis, calycibus fpathaceis. Fl. Juec. 227. Sp pl. 329. Mill. ic. 139. f. 2.

Gentiana major lutea. Baub. pin. 187.

xoc. Alpes Helveticx. Perennis, manfuets. PHARM. GENTIANAE RVBRAE Radix, EJ/Jentia. CVAl. amarifima, lutea. Trita, preftans. vis: balfamica, tonica, fomachica, anthelminthica. vsvs: Cachexia, Arthritis, Podagra, Icterus! Febres intermittentes! Calculus, [Vlcus.] comp. Andromach. Diatellar. Mithridat. Diafcord. Orvietan. Acet. Theriacal. 
198. G,ENTIANA Centaurium corollis quinquefidis infundibuliformibus, caule dichotomo, piftillo fimplici. Fl. Juco. 232. Sp. pl. 332. Fl. dan. t.617. Centaurium minus. Bailh. pin. 278 .

ıac. Europx humidiufcula. Amnua, vulgaris. PHARM. CENT $A V R I I$ MINORIS Herba, (Summitates florentes, ) Extractum, $\nabla, \Omega, \Theta$; Flo. res, Conferva.

CYAL. amarifima. ris: balfamica, tonica, Atomachica.

rsvs: Cachexia, Icterus, Tertiana, Podagra, Hypochondriafis, Scabies.

cosip. Andromach; Acet. theriacal.

199. GENTIANA Amarella corollis quinquefidis hypocrateriformibus fauce barbatis. Fl. Juec. 229. Sp. pl. 334. Fl. dan. t. 328. [Scbmidel. fafc. gesn. 3. p. 45.t.21. f. 87.]

Gentiana pratenfis, flore lanuginofo. Baub. pin. 188 . Loc. Europæ prata ficca.

PHARI. GENTIANELLAE Herba.

CHA. amara.

vis: balfamica, tonica.

vsvs: Heuritis, 'Tertiana! Cachexia.

COMP.

\section{ER Y N G I V M.}

300. ER YNGIVM fotidum foliis radicalibus lanceo: latis ferratis; Horalibus multifidis, caule dichotomo. Syjt. nat. 13. p. 223*. Sp. pl. 336 .

Eryngium americanum foctidum. Herm. lugdb. 236 . t. 237 .

Loc. Americx calidiora.

Perennis, fera. PHARM. IERYNGII FOETIDI Herba, Infusum. QVAl. tetra, fotidiflima, tenax. Eximia. F 4 VIS : 198. [CENTAVRIVM MINVS potius ad Chironic, quam Gentia. nx genus referendum eft.] 
vis: purgans, diaphoretica, diuretica, emmenago. ga, antihy fterica.

vsvs: Hylteria, Febres, Hydrops.

COMP.

20I. ER Y NGIVM campeftre folits radicalibus ample. xicaulibus pinnato-lanceolatis. Sp. pl. 337. H. dan. t. 554. Jacq. aultr. t. 155.

Eryngium vulgare. Baub. pin. 386.

Loc. Germania, Italix, Hifpanix campi ficci.

Perennis, manfueta.

PHARM. ER Y NGII Radix, condita, Herba.

QYAL. Rubaromatico-aquola. Rarior, Hb. Superfua. vis : diuretica, emmenagoga, aphrodifiaca.

vsvs: Calculus, Atecnia.

comp. Radices $V$ aperientes minores.

\section{S A NICVLA.}

202. SANICVLA europera foliis radicalibus fimplicibus, flofculis omnibus feflilibus, Fl. Juec. 235 . Sp. pl.339. Fl. dan t. 283.

Sanicula officinarum. Baub. pin.319.

ıоc. Europx filvx umbrofx. Perennis, frequens. PHARM. SAN ICVLAE Herba.

QVAL. fubinfipida, inodora.

vis: refolvens, adfringens, vulneraria.

vsys: Vulnera.

COMP.

\section{BVPJE E R VM.}

203. RVPLEVR VM rotundifolizm involucris univerfalibus nullis, foliis perfoliatis. Hort. upj. 64 . Sp. pl. 240 .

Perfoliata vulgatifima $f_{0}$ arvenfis, Baub. pin.277. Loc. Europa autralioris agri. Amma, cicur. 
PHARM. PERFOLIATAE Herba, (O), Semina, (D). Q.AL. lubaromatica. Exoleta, dubia, Juperflua. vis: lubadfringens, vulneraria.

vsve: Hernia.

COMP.

\section{TOR DYLIVM.}

304. TORDYLIVM officinale involucris partialibus longitudine florum, foliolis ovatis laciniatis. Hort. cliff.90. upp. 58. Sp. pl. 345. Jaacq. bort. 1. t. 53 . Seleli creticum minus. Baub. pin. I6r.

loc. Monfpelii, Romx, Meflanx. Annua, cicur. PHARM. SESELI CRET ICI Semina.

QYAL. aromatica, debilis. Exoleta, fuperflua. vis: diuretica, carminativa.

vsve:

Comp. Andromach.

\section{DA VCVS.}

205. DAVCVS Carota Ceminibus hifpidis, petiolis fubtus nervofis. Hort. cliff. 89. Fl. fuec. 273. Sp. pl. 248.

Paftnaca tenuifolia fylveftris diofcoridis, vel daucus officinarum. Baub. pin. 151.

B. Paltinaca tenuifolia fativa, radice lutea vel alba. Baub. pin. 151.

LoC. Europx campi.

Biennis, cicur. PHAR.r. DAVCI SYLVESTRIS Semina. SA TIVI [Radix, 7 Semina. QVAL. aquolo-aromatica, fragrans. Rarior. ves: nutricns, diluens, humectans, aperiens, carminativa, diuretica, emmenagoga, anthelminthica. vsvs: Stranguria, Calculus, Lumbrici, Carcinoma. comp. Sem. IV calida minora. 


\section{CONIVM.}

206. CONIVM maculatum feminibus friatis. Fl. Juec. 238. Sp. pl. 349. Facq. auftr. 2. p. 36. t.156.

Cicuta. Dod. pempt. 46r. Riv. mon. 75. [Stcerk fuppl. 2. t. I.]

Loc. Europx agri.

Biennis, rulgaris. PHARM. CONII [CICVTAE TERRESTRIS I. VVLGARIS ] Herba, Succus, injpiffatus, Extractum.

QVAL. tetra.

Vfitata.

vis: narcotica, diuretica, fuppurationem promo. vens.

vsvs: Scirrhus, Scrophula, Carcinoma? Vlcus, Scabies, Gonorrhœa, Leucorrhara, Atecnia. COMP.

\section{A THA MANTA.}

207. ATHAMANTA cretenzis foliolis linearibus planis hirfutis, petalis bipartitis, feminibus oblongis hirfutis. Sp. pl. 352. Gacq. auftr. I. t. 62.

Libanotis foliis tenuilline pinnatis: laciniis petiolatis. Hall. belv. $45 \mathrm{I}$.

roc. Alpes Helvetia, Aufrix. Perennis, cicur. PHARM. DAVCI CRETICI Semina.

CVAL. acris, aromatica.

Rarior.

vIs: carminativa, diuretica, emmenagoga.

vsvs: Flatulentia, Tullis, Calculus.

conp. Andromach. Mithridat. Philonium, Elect. lauri.

\section{PEV CEDA N VM.}

208. PEVCEDA NVM offlinale folis quinquies tripartitis filiformibus linearibus. $S p$. pl. 353 .

Peuce-

286. [CONII loco non debent colligi Cicuta virofa, Aethufas

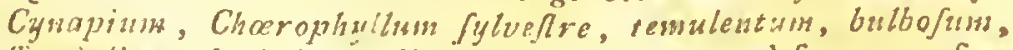
Tordylinn Anthrifcns alizeque umbellatie, quod fæpe non fine damno xgrotorum fakum effe conftat. ] 
Peucedanum germanicum. Baub. pir. 149.

LOC. Gallix, Anglix, Germanix prata. Perennis, cicur: PHARM. PEVCEDANI Radix.

RrAL. calida.

Superfina.

Vis: refolvens, nervina, thoracica, diuretica, emmenagoga.

vsis: Vlcera, Odontalgia, Soporofi, Hemicrania? comp.

\section{FER V L A.}

a०9. FER VLA $A \int a$ fretida foliis alternatim finuatis obtufis. So pl. 356.

Afla færida difgunenfis umbellifera leviftico affinis. Kæuіpf anirn. 535.t. 536 .

LOC. Perfia.

PHARM. ASSAE FOETIDAE Gummi.

Ryal. Gummi-refina, orgaftica alliata. Trita, eximia. vIS: refolvens, carminativa, pellens, diaphoretica, emmenagoga, antifpasinodica, repellens, anthelminthica.

vsvs: Hyfteria, Tympanites, Afthma, Sphacelus, Pạrorychia, Bubo, [Caries!]

comp. Trocb. myrrhx. Empl. matricale.

\section{A SERPITIVM.}

210. LASER PITIVM latifolium foliolis cordatis incifo-ferratis. Fl. Juec. 242. Sp. pl.356. Facq. auftr. 2. t. 146 .

Libanotis latifolia altera f. vulgatior. Bauh. pin. 157 . Łoc. Europx prata arida. Perennis, vulgaris. PHARM. GEN'T IANAE A I.BAE Radix.

QVAL. amara, acris, aromatica, calida.

Rarior, preftans.

vis: tonica, refolvens, diurctica, emmenagoga, ftomachica. 
vsvs: Tertiana.

COMP.

211. L A S E R P I T I VM Siler foliolis ovali - Janceolatis integerrimis petiolatis. Hort. cliff. 96. Sp.pl. 357. Facq. auffr. 3. t. 145 .

Ligufticum quod Sefeli officinarum. Baub. pin: 162. coc. Helvetix, Aufrix, Pilix montes. Rarior. PHARM. SILERIS MON'TANI Semina.

Qval. Cumini odore et fapore remilliore.

Exoleta, ruperfura.

vis: carminativa, diuretica, emmenagoga, [flomachica.]

vsvs: Colica lochialis.

comp. Eleet. lauri.

\section{HER A CLEV M.}

212. HER ACLEVM Spbondylium foliis pinnatifidis. Fl. fuec. 243. Sp. pl. 258.

Sphondylium vulgare hirfutum. Baub. pin. 157 .

цoc. Europx prata. Peremis, vulyaris. PHARM. BRANCAE VRSI (SPHONDYLII) Herba. QVAL. fubaromatica, dulcefcens.

V15:

vsvs: Dyfenteria.

COMP.

\section{LIGVSTICVM.}

213. LIG V S T I C VM Levificum foliis multiplicibus: foliolis fuperne incifis. Hort. cliff. 97. ups. 62. Sp.pl. 359.

Ligulicum vulgare. Bauh. pin. 157.

цoc. Alpes Baldi, Apenninx. Perennis, cicur. pharm. LEVISTICI Radix, Herba, Semina, Extraitum, EfJentia, $\nabla: \Omega, \circ$. 


\section{PENTANDRIA. DIGYNIA.}

Cral. tetra, calida, acris. Sem. urinaria nigra. VIs: alexiteria, pellens, carminativa, exanthematica, emmenaguga.

vsrs: Hyfteria.

conp. Syr. Artemilix.

\section{A NGELICA.}

2I. ANGELICA Arcbangelica foliolorum impari lobato. Fl. lapp. 101. Juec.245. Sp. pl.360. Fl. dan. t. 206 .

Angelica fativa. Baub. piin. 155 .

Loc. Alpes, pracertim Lapponica, Pyrenæx.

Biennis, cicur.

pharm. ANGELICAE SATIVAE Radix, $\nabla$; Herba, Semina, $\Omega$, 0 , Balfamus, Extractum.

QYAL. acris, amaricans, fragrans, aromatica, calida. Trita, preftans. vis: alexiteria, refolvens, ftomachica, fudorifera, carminativa, emmenagoga.

vsvs: Phlogofes exanthematicx, Stomacace.

comp. Orvietanum.

215. ANGELICA Sylveftris foliolis xqualibus ovato. lanceolatis ferratis. Fl. fuec.246. Sp.pl.36r.

Angelica fylveftris major. Baub. pir. II5.

zoc. Europx nemora humida. Perennis, vulgaris. PHARH. ANGELICAE SYLVESTRIS Radix. QV.s. precelentis, fed debilior.

Exoleta. vis: precedenti fimilis in non omnibus.

vsv's: Hyftcria.

comp.

\section{S I VM.}

216. SIVM Ninfi foliis ferratis pinnatis: rameis ter. natis. Sp. pl, 261. Burm. ind.74. 2.29.

Sila. 


\section{4 PENTANDRIA. DIGYNIA.}

Sifarum montanum corxenfe, radice non tubero. fa. Kempf. amwn. 817. t. 818.

zoc. Japonia.

PHARM. NINSI Radix.

QVAL fubdulcis, amaricans, fuavis, aromatica, flavefcens.

$v$ Is: analeptica, inpinguans, cordislis. Rarior, pretiofa. vsvs: Atecnia, Debilitas, Marafmus, Senectus?

\section{SISON.}

217. SISON Ammi folis tripinnatis: radicalibus li. nearibus; caulinis fetaceis; ftipularibus longiori. bus. Sp. pl. 363. Focq. hort. 2. t. 200.

Ammi parvum, foliis forniculi. Baub. pin. 159.

Loc. Apulia.

PHARM. AMMIOS VERI Semina.

Annur, frbmarrfueta.

QVAL. amaricans, aromatica.

vis: fudorifera, carminativa.

Rarior, preftans.

vsvs: Sterilitas.

comp. Sem. IV. calida minora, Andromach.

\section{BVBON.}

218. BVBON maredonicum foliis rhombeo - ovatis crenatis, umbeliis numerofiflimis. Hort. cliff. 95. up $\int .62 . S p . p l .364$.

Apium inacedonicum. Banı.pin. 154. non Smyrnium. Loc. Macedonia? per Sinyrnam. Perennis, manfueta. PHARM. PETROSELINI MACEDONICI Semina. QVAL. aromatica, dulcefcens. Rarior, fuperfua. VIs: carminativa, diuretica, emmenagoga.

vsvs:

comp. Andromach.

219. $B V_{\text {. }}$

217. [ Sifon Amomum, Jacq. Kort.3. t. I7. Officinis pharmaceuticis dat AMOMI V VLG. Semen.]. 
219. R BON Galbanum foliolis rhombeis dentatis friatis glabris, umbellis paucis. Hort. clif. 9 \%. Sp. pl. 36.t. Mant. 335." Facq. bort. 3.t.35. Anifum africanum frutelcens, folio anifi, galbani. ferum. Pluk, alm.31.t. I2. f.2. toc. Aethiopia.

Perennis, fubmanfueta. FHARM. GALBANI Gummi commune, repuratum, Efentia, OO.

Ryal. Refma graveolens, amaricans. Trita, preftans. vis: anodyna, antilpafmodica, pellens, carminativa, emmenagoga, expectorans, repellens, ma. turans.

vsvs: Afthma, Hyfteria, Tumores.

comp. Andromach. Mithridat, Diafcordium. $V_{n g}$. apoftol. Vng. althrx. Empt. galban. Empl. diachyl. gummof. Empl. mucilagin. Empl. điaphoret. Empl. oxycroceum. Empl. Atypticum.

\section{CVMIN VM.}

220. CVMINVM Cyminum. Sp. pl.365. Riv. pent. Cuminum femine longiore. Baub. pin. 146. Loc. Aegyptus; in Melita profertim feritur.

\section{Amnua, manfueta.}

PHARM. CVMINI Semina, $\nabla, \Omega, 00$. QVAL. acris, aromatica. Rarior, preftans. vis: carminativa, diuretica, emmenagoga.

vsrs: Colica flatulenta, Hyfteria, Tympanitis, [Tumores frigidi.]

coMP. Sem. IV calida majora. [Sacculi refolventes.]

\section{CICVTA.}

22r. CICVTA virofa umbellis oppofitifoliis, petiolis marginatis obtufis. Fl. fuec. 253. Sp. pl. 367 . F. dan. t.208. Gunn. norv. 1. p.26. t.2. f. ..2. 
Sium erucx folio. Baulb. pin. 154.

цос. Europx paludes. Peremis, vulgaris. pharm. CicVTaE [AQVATICAE] Herba. QVAL. acris, feptica, tetra. I Rarior, cauta. vis: narcotica, hypnotica, repellens.

vsvs: Bubo, Carcinoma, Lienis infarctus, Herpes. comp. Limpl. cicute.

\section{A ETHVSA.}

222. AETHVSA Meum foliis ommibus multipartitofetaceis. Syft. nat. 13. 237. Facq. auftr. 4. t. 303. Meum foliis anethi. Bubib. pin. 148 .

Ioc. Montes Italix, Gallix, Helvetix, Germanix. Perennis, cicur.

PHXRM. MEV Radix.

QVAL. acris, aromatica, calida.

vis: carminativa, diuretica, emmenagoga.

vsus: Tertiana. Afthma, l.eucorrhoca. comp. Andromach; Mithridat.

\section{CORIA NI)R VM.}

223. CORIANDR VM fatinum fruchibus gilobofis. Hort. cliff. 100. Hort. upf. 63. Sp. fl.367.

Coriandrum majus. Baub. pin. 158 .

\&.oc. Europæa agri.

pharm. CORIÃNDRI Semina, Confectio.

gval. tetra, fufpecta. Cullinaris, rarior. vis: hypnotica, carminativa, lactifuga.

vsvs: Hytteria, Tertiana.

COMP.

\section{SCANDIX.}

224. SCANDIX Cerefolium feminibus nitidis ovato. fubulatis, umbellis feflilibus lateralibus. Fi, juec. 255. Sp. pl. 368. Jacq. aufit. 4. t. 390 .

Charo. 
Chrophyllum fatioum. Baub. pin. 152.

loc. Europio arri.

Seribfiris, cicur. PHAR. CEREFOLII Herba, Aqua.

QV.A. aromatica, dulcelcens, oleracea, crepitans.

Culinaris, uftata.

ris: antiplogiftica, refolvens, aperiens; mundificans, diurctica, lackifera, vulneraria. rsvs: Hydrops, Tullis, Hemoptylis, Phthifis,

Vertigo, Hamorrhois:

comp.

\section{CHAEROPHYLT VM.}

225. CHAEROFHLLVM fylveftre caule levi friato; geniculis tumidiulculis. Fl. faec.257. Sp. pl.369. Facq. auftr.2. t. 149 .

Myrhis fylveftris, Reminibus lrvibus. Baub pin. I60. Loc. Europx pagi et pomaria. Pereninis, vulgatijsma. PHARM. CICVTARIAE Herba:

CVAL. amaricans, fublotida: Exoleta, fuperfiut. vis: fubvenenata? dubia.

vsres: Gaigrena:

Cosis:

\section{IMPERATORIÁ.}

226. INPERATORIA Oftrutbium. Hort.cliff. 10j up $.65 . S p \cdot p l .37 \mathrm{x}$.

Oftruthium. Dot pempt. 514 .

Loc. Auftrix montes.

PHARM. IMPERA TORIAE Radix.

Perennis, cicur. Qral. anaro-acris, aromatica, calida. Trita, preftans. VIs: apophlegmatizans, carminativa, fudorifera, cmmenagroga.

vors: Flatulentia, Hyferia, Colica, Serpigo, Sterilitas, Tcutiana, Haralyfis.

ron?. Orvietunum; Act. theriacale:

(j) PASTI. 


\section{PASTINACA.}

227. PASTINACA fativa foliis fimpliciter pinnatis. Hort. cliff. 105. Hort. upp. 66. Il Juec. 259. Sp. pl. 376.

Paftinaca fylveftris latifolia. Baub. pin. 155 .

Loc. Europa.

Biennis, cicur, nleracea.

PHARM. PASTINACAE Radix, Semina.

QVAL. aquofa. Culinaris, $\mathrm{Rd}$. fuperfura. vis: diluens, humectans, nutriens, flatulenta, aphro. difiaca, diuretica.

vsvs: Tertiana.

COMP.

228. PASTINACA Opopanax folis pinnatis: foliolis bafi antica excifis. Sy/t. nat. 13. p.240. Sp. pl. 376. [Gouan. illuftr. 19. t.13. 14.]

Panax coftinum. Baub. jin. 156.

zoc. Galloprovincia, Italia, Sicilia. Perennis, fubcicur. PHARM. OPOPANACIS Gummi.

CYAL. Gummi-refina amaricans, acris, graveolens, intus Alavefcens, extus rufefcens. Rarior. vis: nervina, eccoprotica, carminativa, cmmena. goga.

vsvs: Tuflis fenilis, Afthma humorale, Paralyfis. comp. Andromach. Mithridat. Hiera colocynth. Trncb. myrrha, Vng. apoftolorum, Empl. mucilaginofum, Empl. flypticum, Empl. manus dei, Pilulce fortidx.

\section{ANETHVM.}

229. ANETHVM graveolens fructibus compreffis. Hort. cliff. 1o6. Hort. up. 66.

Anethum liontenfe. Baub. pin. 147.

上oc. Hifpania, Lufitania.

Annua, cicur.

PHARM. 
PHARM. ANETHI Herba, $\nabla$, ( ), Flores, Semi. na. 0 .

Q.A.. tetra, libaromatica. Callinaris, trita, preftans. vis: hypnotica, caminativa, lactifera.

vsvs: Agrypnia, Culica, Singultus! Vomitus.

comp. Flores IV cammantes. Emol. ranarum mercuriale, Empl: nucilagin.

æ30. ANETHVM Fenicuirm fructibus ovatis. Hort. cliff. 106. Hort. cliff. 66.

Fceniculum vulgare germanicum. Baub. pin. 447. Loc. Gallia. Biennis, manficta. pharr. FOENICVL, I Radix, Herba, Aqza, Semina, Confectio, $\nabla / \mathrm{mm}$. $\Omega$.

QV Al. aromatica dulcetcens. Culimzris, trita, praftans. vis: relolvens, pellens, carminativa, lactifera.

vsvs: Ophthalmia! Vomitus. ['Tomina infan. tum.]

conp. Sem: IV. calida major. Rad. V: aperientes majores. Syr. V radicum, Andromach. Mithris dat. Philonium, Diaphenic. Benedicta. Syr. ar. temifix; Syr. prafit; Syr. hyllopi. Pil. aurex; Ha= mech.

\section{CARVM.}

23i. CARVM Carvi. i.t. fuec.260. Sp.pl 378. Faca. auftr. 4. t.393.

Cuminum pratenle, Carvi officinarum. Baub. pin. 158. Loc. Furopie prata. Biennis, vulgaris. IHarm. CARVI Semina, Confectio, $\nabla, \Omega, 00$. QVAL. acris, aromatica, calida. Cillinaris, jrejtans. Vis: relolvens, carininativa.

vers: Codica, Tertiana.

con:. Sem. IV calida majora; Elect. lauri; 


\section{PIMPINELLA.}

232. PIMPINELLA Sarifraga foliis pinnatis: foliolis radicalibus fubrotundis; fummis linearibus. Fl. fiuec.261. Sp. pl.378. F\% dan. t.66g. Facq. auft.r. 4. t. 395.

Pimpinella faxifraga major altera. Baub. pin.r6o.

Loc. Europa campi. Perenis, vullgatifima.

pharm. PIMPINELLAE NOSTRATIS '[ALBAE]

Radix, Conferva, Efjentin, $\nabla$; Herba, Semina. CVAl. acerrima, hircina, calida.

Trita, profians, Stabliana.

vis: refolvens, diaphoretica, diuretica, emmenagoga.

vsvs: Coryza, Dyfpnaa, Angina ferofa, Hyfteria.

COMP.

233. PIMPINELLA Anifun foliis radicalibus trifidis incifis. Sp. pl. 279 .

Anifum herbariis. Baub. pin. 159.

loc. Syria, Aegyptus. Ammu, manjucta. PHARM. A NISI Semina, $\nabla, \Omega, 00$.

QVAL, aromatica dulcefcens, calidiuficula.

Culinaris, ufitata.

vis: refolvens fangutinem et lac, becchica, carminativa.

vsvs: Tormina, Tuflis.

comp. Sem. IV calida majora, Pil. aurex, Conf. hamech, Balf. fulphuris anilatus.

\section{$\triangle P I V M$.}

234. APIVM Petrofelizum foliis caulinis linearibus, involucellis minutis. Hort. cliff. 108. Hort. 2 p S. 67. Sp. pl.379. 
Apium hortenle f. Pctrolelinum vulgo. Baub. pin. 153. Loc. Sardinia. Biennis, oleraica, cicur. pHAR. PETROSELINI Radix, Herba, $\nabla$, Se. mini, 0 .

cilis aromatica, culinaris. Preftans. vis: relolvens, nutricus, diuretica, emmenagoga, lackifuga.

vsis: Icterus, Dyfuria, Contufura, Ablactatio, Pediculi.

comp. Syr. V radicum; hyffopi.

235. A PIVM graveolens foliis caulinis cuneiformibus, umbellis ferilibus. Fl. Juec. 262. Sp. pl.379. Fl. dan. t. 790.

Apium paluitre $\int$. Apium officinarum. Batbb.pin. 154 . I.oc. Europe paludes.

Biennis, cicur. PHAR. A PII Radix, Herba, Semina.

QYa. fubtetra, dulcefcens, fufpecta. Cuinaris, rarior. vis: camminativa, pellens, fudorifera, diuretica, emmenagoga, repellens.

vsvi: Febres. noxia Apoplecticis, Epilepticis, Vertiginofis, Caliginofis, Pediculis.

comp. Sem. IV calida minora, Radices V aperientes majores, Syr. V radicum, Syr. hyllopi, Pil. aurex.

\section{$T R I G Y N I A$. SEMECARP VS.}

236. SFMFCARPVS Anacardium. Suppl. pl.182. Anacardium primum. Baub. pin. 5 II.

zoc: Indix orientalis deferta \& fylvatica arida. Arbor, peregrinc. 
PHARM. ANACARDII ORIEN'TALIS Fructus.

CVAl. muclei dulcis (amygglali), putarimis acer. rima.

Exoleta.

vis: muclei nutriens, putrminis cauftica.

vsrs: Morolis, Oblivio.

COMP.

\section{RHVS.}

237. RHVS Coriaria foli's pinnatis obtufiufcule ferratis ovalibus fubtus villofis. Hort. cliff. 106. ups. 68. Sp. pl. 379 .

Rhus folio ulmi. Banth. pin. AIA.

s.oc. Galli:, ltalia. Arbor, manfueta. PHARM. SVMACH Flores, Semina.

e'An.. ftyptica, fubremenata. Rarior, cauta. vis: adfringens, coriaria, refrigerans.

vsvs: Diarrhoca, Dyfenteria, Stomacace, Rhiagas.

COMP.

238. RHVS Vernix foliis pinnatis integerrimis annuis opacis, periolo integro aquali. Syft. nat. 13. f. 242. Sp. pl. 380. ['libunb. jap. 121.]

Sits vel Sitz - diu. Kumpf. rman. 79r. t. 792.

上oc. Japonix, Virginix paludofa. Arbor, manfueta. PHARM. VER NICIS Refina, (O).

QVAL. toxica.

Externa.

VIS :

vsvs: Hxmorrhois.

coßي. Viggu. martiatum.

\section{ASSINE.}

239. CASSINE Peragua foliis pitiolatis ferratis elli. pticis acutiufculis, ramulis ancipitibus. Mant. f. 2. p. 220.* Mill. it. t. 83. f. I.

2;9. PERáavan alii pro fpecie Clevoderadri habent. 
CaRinx vera perquam fimilis arbufcula, phillyrea foliis antagoniltis. Pluk. mant. 46. t. 371. f. 3 . цoc. Carolina, Virginia. Arbor, manfueta. PHARM: PERAGVAE Folia. QYA. amara. Inufitata.

vis : vomitoria, purgans, fudorifera. visus: Diabetes! Colica calculofa. COMP.

\section{SAMBVCVS.}

340. SAMBVCVS Ebulus cymis tripartitis, ftipulis foliaccis, caule herbaceo. Fl. fuec. 266. Sp. pl.385. Sambucus humilis f. Ebulus. Baub. pin. 456 . t.oc. Europa.

Perennis, cicur.

pharm. EBVLI Radix, Cortex interior, Folia,

Flores, Pacce, Rob, Semina.

QVAL. amaro-acris, tetra foctida, naufeofa.

Rarior, eximia, caute. vis: narcotica, anodyna, pellens, fudorifera, diu. retica, emetica, draflica.

vsvis: Hydrops, Leucorrhoca, Scabies.

comp. Extr. panchymagog. Empl. ranar. mercuriale.

24I. SAMBVCVS nigra cymis quinquepartitis, caule arboreo. Fl. Jucc. 265. Sp. pl. 385. Fl. dan. t. 545 .

Sambucus fructu in umbella nigro. Bawh. pin. 456 . Loc. Europa.

Arbor, cicur. pharar. SAMPVCI Cortex interior, Folia, Flores, Conferva, $\nabla, \Omega$, t, Baccx, Rob, $\Omega$, Semina, (0).

Ryar. acris, tetra fotida, naufeofa. Trita, eximia. vis: narcotica, anodyna, pellens alvum, fudo. rons, arinam, menftrua, lac. 
IO4 PENTANDRIA. TETRAGYNIA.

vsvs: Eryfipelas, [Febres, Rheumatifmus, Arthritis, Exanthemata, ] Fl. Rob. Dy fenteria Bacc. Hycrops, Varix ani! Cort.

comp. Spec p. cataplafmate.

\section{T A MARIX.}

242. TAMARIX gallica lloribús pentandris. Hort. cliff. I11. Sp. pli 386. Niill ic. t. 26́2. f. 1.

Tantarix altera five folio tenuiore, gallica. Baub. pit 485 .

tor. Gallia, Hifpania, Italia. Arbor, manfueta. IHARM. 'T $\triangle M A R I S C I$ Cortex (radicis), Lignum? Folia.

CrAc. Ityptica.

vis: adfringens, coriaria, obftipans.

vsvs: Hypochondrialis, Stomacace, Epiphora, Prolluvia.

COMP:

\section{TETRAGYNIA. PARNASSIA.}

343. PAR NASSIA paluftris. Fl. Juec. 268. Sp. pl. 391. Fl. dan. t. 584.

Gramen parnalli, albo fimplici flore. Bauh. pin. 309. Loc. Europre pra a fublumida. Peremis, vulgatifina. JHARM. HEPATICAE ALBAE Herba, Flores.

QVAl. amaricans.

VIS : fubadfringens.

vsve: Cardialgia?

COMP. Exoleta, dubia, juperfua.

PEN-

242 [TAMARISST Cort. e Tamavicis germnica rad. detraheudum elle, monent Pharm. anfir. prov.] 
PENTANDRIA. PENTAGYNIA. IOS

\section{PENTAGYNIA. STATICE.}

244. STATICE Linonim feapo paniculato tereti, foliis lavibus enerviis fubtus mucronatis. Fl. fuec. 270. Sp. pl. 394. Fi. dan. t. 315.

Limonium maritimum majus. Bazb. pin. 192.

Loc. Arabia.

PHARI. HEHEN RVBRI Radix.

Dubia planta.

QYaL. firptica.

vis: adfiringens,

vsrs: Debilitas, Hrmorrhagia,

comp. compofita arabum.

\section{LINVM.}

245. LINVM ufitatiffunum calycibus capfulisque mucronatis, petalis crenatis, foliis lanceolatis alternis, caule fubfolitario. Sp, pl.307.

Linum fativum. Bath. pin. 214.

roc. Pannonia, Hilpania. Ammun, cicur, cerealis. PHARM. liNI SATIVI Semina, (O).

RAL. pinguis:

vis: relaxans, obtundens, cmolliens.

vsvs: Arthritis, Calcules, Colica, Hamoptyfis, [Oblipatio, Iliaca, Heuritis. Sem. 'Tumores, Hernia incarc. Angina, Ifchuria.]

covip.

246. LINVM catbarticum foliis oppofitis ovato- lanceolatis, caule dichotomo, comllis acutis. F. fuec. 27 1. Sp. $/ 2.4 \mathrm{Cr}$. F\% dan. t. 85 \%.

Linum pratente, flofculis cxignis. Barb. pin. 214 .

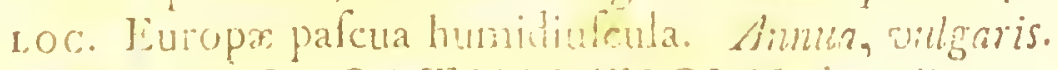
PHARM. LINI CATHARTLCI Lorba jij.

$$
\text { G } 5 \text { (?... }
$$


ICE PENTANDRIA. PENTAGYNIA.

Cyar. amara, naufeofa. Rarior, prejzans. ris: purganls.

vsrs: Nephritis, Calculus, Hydrops.

cOMP.

\section{1) ROSERA.}

247. DR OSERA rotundifolia fcapis radicatis, foliis orbiculatis. Fl. fuec. 237. Sp. pl. 402.

Ros folis folio rotundo f. oblongo. Bauh. pin. 357 . ıoc. Europa paludes. Biennis, vulgais. HHARM. RORIS SOLIS Herba. CYAl. fubacris, Jactaria. vis: corroderis, fufpecta. rsve: Verruca? Clavus?

Exoleta, dubia. conis, 


\section{se \\ CLASSIS VI.}

H E X A N D R I A. MONOGYNIA.

BROMELIA.

248 BROMELIA Ananas foliis ciliato-fpinofis mucronatis, fpica comofa. Sp. pl. 408.

Anaias. Comm. bort. 1. p. 109. t. 57. Loc. America meridionalis. praris. ANANAS Fructus, Cortex exterior [recens].

QYAL. acris, ambrofiaca.

vis: emmenagoga!

Inufitata.

vsvs:

comp.

\section{A LLIV M.}

249. ALLIVM Porrum caule planifolio umbellifero, faminibus triculpidatis, radice tunicata. Hort. $11 \int .77 . S \% \cdot p l .423$.

Porrum commune capitatum, Baub. pin. 72.

I.OC.

Biemis, cicur, oleracea.

YHARM. PORR J Semina.

RVA. acris fubdulcefcens, orgaftica. Exoletn, culin. vis: calefaciens, emmonagoga, diuretica, diaphoretica.

vsvs: Calculus.

comp.

250. ALIIVM Victoralis caule planifolio umbellifero, umbella rotundata, flaminibus lanceolatis corolla longioribus, foliis ellipticis. Sp pl.424. Far?. auflr. 3.t. 216. 
IO3 HWNANBRIA. MONOGYNIA.

Allium montam.m latifolium maculatum. Baillopin. ${ }^{-} 4$. I.nc. Alpes Helveticx.

Perennis, fubminufueta. IHARM. VIC'TORIALIS LONGAE Ravir.

QVAL. acris orgaftica.

vis: Allii fequentis.

Exoleta, Jiserfluca.

rsvs: fuperfitiofus, magicus,

comp. Amuleta.

251. Al.LIVM fatioum caule pianifolio bulbifero bulbo compofito, ftaminibus tricufpidatis. Hort. ups. $70 . S p \cdot p l .425$.

Alliuin fativum. Bawh. pin. 73 .

Loc. Sicilia. Premis, cicur, oleracea. PHAKII. ALLII Radix, $\nabla,+$.

(Qу A.. acris, fubcauftica, organtica, lactaria, urinaria.

Culinaris, preficans.

vis: excitans, pellens, maturans, antlelminticica,

dinetica, diaphoretica, alexitcria.

vsus: Hylferia, Colica, Hydrops, Calculus, Tuf. fis, Clavus, [T Tenia].

comp. Oxymel alliatum.

352. ALl.iVM Cepa fapo nudo inferne ventricofo longiore foliis teretibus. Hort.upf: 77. Sp.pl. 431. Cep vulgaris. Baub. pin. ZI.

LOC.

Biennis, cicur, oleracen. PHARM. CEPAE Radix.

CyAL. acris, orgaftica. Culinaris, preftans. vis: maturns, diuretica, diaphorctica, alexiteria, Hatulenta, aphrodifiaca, anthelminthica.

vsvs: IIrmorrhagia, Calculus, Otalgia, Bubo, una, Cophofs, Varix ani.

COMP.

250. VICTORIALIS IONGAE defciu fubritui poteft ALLIVM i) Nintin. 


\section{LIJIVM.}

253. LILITM candium Liits fparfis, corollis campanulatis intus chlabris. Hort. cilff. 120. upf. 80. Sp. fit. 433 .

Lilimm albun, hore ered?o, vulgare. Baub. pin. 75 . soi. Sria, Palatina.

Poremis, cicur. phaser. Llill ALBI Radix; Flores, Comerva,

$\nabla$, (ㅇ); Anther:

QYAl. I\%, fragrans. Rd. mucilaginola. Trite, preftuns. vis: emolliens? maturass, onodyna, cuth. emmeiñogga.

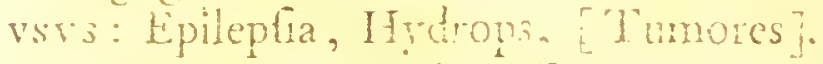

cove. Jimpl mucagin. Lmpl. lanar. increurale. Clyilures, Cataplafmata.

\section{SCILLA.}

254. SCILLA maritima nudifiora, bracteis refractis. Sp. pl. 442 .

Scilla vulgaris, ractice rubra. B.zub. pin. 73 .

Loc. Hifpanix, Sicilix, Syrix littora arenofa.

Peremis, fubmanfueta.

PHARM. SQVILLAE Radix, Acetum.

Cyal. acris, amara, orgaftica, cruda corrofiva.

Trita, beroica.

vrs: incidens, flimulans, pellens, diaphoretica, diuretica, emetica, hydragoga, expectorans.

rsve: Afthma, Tufis, Cachexia, Hydrops t ICte.

rus:, Sopores.

coss. Andromach: Oxymel feilliticum.

\section{ASPIODER, VS.}

255 ASPHODEI,V remofus caule nudo, folits enfiformibus cainatis favibus. Sp pl. 144.

Aphocichus albus ramosus mas. Barbb. prn. 29. 


\section{IIO HEXANDRIA. MONOGYNIA.}

Loc. Hilpanix, Lufitanix, Narbone colles.

PHARM. ASPHOIELI Radix.

Perennis, manfueta. QVAL. ingrata, acris. Dubia, exoleia, fuperfun. VIs: diuretica, emmenagoga, emolliens? maturans. VSVS:

COMY.

\section{ASPARAGVS.}

256. ASPARAGVS officizalis caule herbaceo teret erceto, folitis fetaceis, flipulis paribus. Fl. Juec. 291. Sp. pl.448. Iit. dian. t.805.

Afparagus fylveftris, tenuilimo folio. Baab.pin. 490. 1.oc. Europa autralioris arenola. Peremis, cicur. pharm. ASPARAGI Radix, Semina.

एVAL. aquofa, fubdulcis, inodora, urinaria fo. tida.

Culinaris, u/itata. vis: nutriens, diluens, humectans, debilitans, mundificans, diuctica, aphrodifiaca.

vsrs: Calculus?

comp. Rad. $\mathrm{V}$ aperientes majores, Syr. Althæx.

\section{CONVALLARIA.}

257. CONV ALL A R I majalis fcapo nudo. Fl. fuer. 229. Sp.pl. 451. Fi.dan. t. 854 .

Lilium convallium album. Baub. pin.304.

r.oc. Europre prata dura. Perennis, vulgaris: PHARar. LILII CONVALlavM Flores, Comjerva, $\nabla, \Omega, \tilde{V}$.

CVAL. fragrans, amara, acris, naufeofa. Vfitati. vis: narcotica, antifpasmodica, errhina, purgans. vsvs: Epilcpfia, Apoplexia, Coryza, Epiphora. comp. Spir, Limbiyon. Agre. Hirund. 
258. CONVALLARIA Polygonatum foliis atemis amplexicaulibus, caule ancipiti, pedunculis axillaribus fubunitloris. Fl. Juec.204. Sp. pl. 45r. li. $6072, t .377$.

Polygonatum latifolium vulgare. Baub. yin. 303.

lor. Europx precipitia montium. Teremis, onlyaris. PHARM. SIGILLI SALOMONYS Radix.

erat. fatua, mucilaginolia.

Exoleta, dubia.

rrs: colmetica? vulneraria.

vsve: Sugifillatio.

COMP.

\section{A I.O T}

259. ALOE perfolinta fioribus pedunculatis cernais corymbofis fubcylindricis. Sp. hi. $45 \%$

a. A. vera foliis fpinofis confertis dentatis vaginantibus planis maculatis. Hort. cliff. 13r. Hort. upf. 86. Aloë vulgaris. Baun. pin. 386. Kadanaku. Rbeer. mal. ir. p. 7. t. 3 .

roc. Italia, Sicilian. Peremis, fulmanfueta. PHARM. $\Lambda$ LOES HEPATICAE ${ }^{i} i$, loti $\partial j$, Tinctura, Extractum. $习$ ß.

ß. Alnë americana, ananx floribus fuaverubentibus. Pluk. alm. 19. t. 240. f. 4 :

Loc. Barbados. Perennis, fubmanruta. PHARM. ALOE SVCCOTRINA.

$\boldsymbol{\gamma}$. Aloë guineenfis caballina, vulgari fimilis, tota maculata. Comm. preal. 40.

zoc. Guinea. PHARM. ALOE CABALLINA.

Perennis, fubmanzzeta.

Q 43.10

259. [ F.x hac Specie maxima copia Aloës paratur; modum praparandi, ad promontorium obme fpei contietum vid. in art. Iund. I p. ziz. Triplex Aloës fuecies, in plarmacnpoliis venalis, puritate potius, quan origine, differt ilsonl.

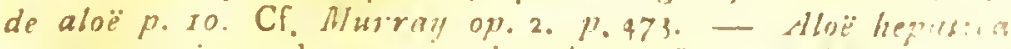
pura et o tima and promontorinim bone fpel ex $A$ lnis jpisia ia, Suppl. 205. obtinetur. Thanb. 6. c.] 
cy. amarifima, nauleola. $\gamma . R a r a ; \alpha . \beta$. Trita, beroicd. vis: purgans, pellens, ballamica, fomachica, an: theminthica, vulneraria.

vsvs: Obftuchiones, Hypochondriafis, Icterus,

Vermes, [Vulnus, Vlcus, Caries].

comp. Aloë rolata. Alö violata. Elix. proprietatis c. t-vel c. 1 jj. Pil. ammoniacx, cochir. poly. chrefix, capitales, francofurtenf. ruff, fuccin. Stanliana Gr. XV. Ine quibus. Extrat. panchy. imag: ITera picra, Vigur. arthanit. Empl. Aticticum:

\section{ACORVS.}

260. ACORVS Calamis. Fl. fucc.297. Sp.pl.426.

a. Acorus verus 1. Calamus aromaticus officinarum. Banl. pin. 34.

Loc. Juropa paludes.

Percnmis, cinur. PHARM. CALAMI VVLGARIS Radix, condita; Evtrizerin, $\nabla, \Omega$.

B. Aconus verus 1 . Calanus aromaticus radice tenuio: re, Herin. $\operatorname{lugdb.9}$.

Loc. India orientalis, Brafilia. Perennis, peregrina: PHARM. ACORI VERI Radix. QNA. arcmatica, acris, amaricans, fragrans.

Vjitata, preftans. vis : incidens, alexiteria! calefaciens, pellens, diuretica, ftomachica.

vsve: Anorexia, Febres exanthematicx malignx! Vertigo.

com.. Andromach. Mithridat. 'Orvietan. Elect. Lau= li, Troch. acori, Troch. capparidis.

\section{ACHRAS.}

26r. ACHRAS Sapota floribus folitariis, foliis ovato: oblongis. Sp.pl. 470. [Scbrepf. mat. med.50] 
Anona foliis laurinis glabris viridi-fufcis, fruclu minore. Sloan. - Catesb. car. 2. p. 87. t. 87. ıoc. America meridionalis. Arbor, peregrina. PHARM. SAPO'TILLAE Semina 3 j - 3 ij. [Cortex.] CVAL. [acris.]

vis: [diuretica, Cort. adnringens.]

vsrs: Colica nephritica, [Dyluria.]

comp.

\section{BERBERIS.}

262. PERPER IS vulgaris pedunculis racemolis, Fl. fleec. 3ir. Sp. pl. 47x.

Berberis dumetorum. Bauh. pin. 454.

s.oc. Europre fylva.

Frutex, cicur. PH.AR. BERBERIDIS Cortex, Baccx, condite, Succus, Syrupus, Rotulce, Semen. Qval. Bacc. acidiffima; Cort. Hava, amara. Preftans. vis: refrigerans, attenuans, antiputredinofa, Cort.

tinctoria, purgans, tonica.

vsvs: Febres putridx, Cholera, Cort. Icterus, Leucorrhœa, Aphthx.

COMP.

\section{$D I G I N I A$. ORYZA.}

263. OR YZ A Sativa. Hort. cliff. 137. Sp. pl. 475.

Oryza. Baul). pin. 24.

Loc. Indix inundata. Annua, fera. PHARM. ORYZAE Semina, $\Omega$. QVAL. farinofa, dulcis. Culinaris, rarior. vis: nutricns. vsve: cibarius. [Diarthoca, Dyfenteria]. 


\section{$T R I G Y N I A$.}

\section{R V MEX.}

264. RVMEX fanguineus floribus hermapliroditis: valvulis integerrimis: unica granifera, foliis ovato - lanceolatis. Sp. pl. 476. Hort. cliff. 138. ups. 89 .

Lapathum folio acuto rubente. Baulb. pin. I15.

Loc. Virginia. Biennis, cicur. pharm. LAPATHI SANGVineI Radix, Succus, Semina.

eVAl. purpurea, auftera. Inufitata.

vsvs: adftringens, refrigerans.

vsvs: Dyfenteria! Vlcus.

COMP.

265. RVMEX acutus floribus hermaphroditis: valvulis dentatis graniferis, foliis cordato-oblongis acuminatis. Fl. Juec. 316. Sp. pl. 478.

Lapathum acutum f.Oxylapathum. Baulb. bift. 2. p. 983. Loc. Europx humentia.

Perennis, frequens. pharm. LAPATHI ACVTI Radix, Herba, Semina. Qval. ftyptica, naufeofa, radicis amarior, lutea, foliorum acidior. Preftans, Hb. Superfun. vIs: adftringens, eccoprotica.

vsvs : Dyfenteria! Scabies! Vlcus.

COMP.

265. RVMEX aquaticus floribus hermaphroditis: valvulis integerrimis nudis, foliis cordatis glabris acutis. Fl. fulec. 315. Sp. pl. 479.

Lapathum aquaticum, folio cubitali. Bauh. jim. 116. LOC. Europe paludes.

Perennis, vulyaris. PHARM. HERBAE BRIT A N NCAE Radix. QVAl. flava, amaricans, auftera. Rarior, eximinia. 
vits: adfringens, refrigerans.

vsrs: Cacoëthes! 'Vlcus malignum, Stomacace!

Hrmorrhagia!

comp. Pulv. dentifr.

267. R V MEX foutatus floribus hermaphroditis, foliis." cordato - haftatis, caule tereti. Hort. cliff. 138 . ups. 89. Sp. pl. 480.

Acetola rotundifolia hortenfis. Baub.pin. II4. Loc. Galloprovincia, Hetruria. Biemnis, cieur. PHARM. ACETOSAE ROTVNDIFOLIAE Herba. QYAL. acida, reliquis debilion. [Acetofa noftrate gran tior minusque auntera].

Exoleta.

vis : refrigerans.

vsvs: Scorbutus.

COMP.

268. R VMEX alpinus floribus hermaphroditis fteri. libus femineisque, valvulis integerrimis nudis, foliis cordatis obtufis rugofis. Sp.pl. 480.

Lapathum hortenfe rotundifolium. Baub. pin. II5.

Loc. Europr humentia.

Perennis, frequens. PHAR. RHABARBARI MONACHORVM Radix, CVAL. amaro - auftera, Hava.

vis: adfringens, fublaxans.

vsve: Vlcus.

COMP.

269. RV MEX Acetofa floribus dioicis, foliis oblongis fagittatis. Ni. fuec. 3r8. Sp. pl. 48 I.

a. Acetcía pratenfis. Baub. pin. II4.

Loc. Europx graminola. Perennis, vulgatiffma. PHARM. ACETOSAE NOSTRATIS Radix, Herba, Conferva, Syrupus, $\nabla$, Semina.

B. Acetofa montana maxima. Baub. pin.ut4.

coc. Alpes varix, Lapponicx. Perenzis, cicur. 
II6. HEXANDRIA. TRIGYNIA.

pharm. ACE TOSAE HISPAN IC AE Radix. QYAL. acido - auftera, amara. Rad. rubra.

vis: refrigerans, tonica, obflipans.

Rarior, preftans.

vsvs: Scorbutus, Profluvia, Febres exacerbante.

- comp. Decocta rubra; Diafcordium; Conf. Hyacinth.

\section{COL, CHICVM.}

270. COLCHICVM auitumnale foliis planis lanceolatis erectis. Hort. cliff. I4.0. Hort. ups. 90. Sp.pl. 485 .

Colchicum commune. Baub. pin. 67.

Loc. Europa aufralioris prata fucculenta.

Peremis, cicurs.

pharm. COLCHICI Radix, Extractum.

QVAL. graveolens, acris. Rarior. vis: narcotica, phantaftica, diuretica, draftica, exanthematica, phthiriaca.

vsvs: Hydrops, Marifca; Amuletum Peftis.

comp. Oxymel Colchici.

\section{TETRAGINIA. PETIVERIA.}

27I. PETIVER I A alliacea floribus hexandris. Hort. cliff. 141. ups. 91. ACt. Jtockb. 1744. p. 287. t. 7. Sp.pl. 486. Trew. ebret. p. 33. t. 67.

Verbenx aut fcorodonix affinis anomala, flore al.

bido, allii odore. Sloan. jam. 64. bift. I. p. 172. roc. Jamaica, Gallia xquinoctialis. Frutex, ferus. pharm. SCORODONIAE Herba.

QVAl. orgaftica, alliacea, lactaria, urinaria. Preftans. vIs: fudorifera, diuretica. vsvs: Febres intermittentes, exacerbantes. COMP. 


\section{(10.}

\section{CLASSIS VIII.}

O C T A N D R I A. $M O N O G Y N I A$.

\section{TROPAEOL VM.}

272. TROPAEOLVM majus foliis peltatis fubquinquelobis, petalis obtufis. Hort. ups. 93. $S p$. pl. 490.

Viola inodora fcandens, nafturtii fapore, maxima odorata. Herm. lugdb. 628 .

loc. Peru. Perennis, nobis anmua, cicur. PHARM NASTVRTII INDICI Herba.

R.Al. aquofa, acris, oleracea. Exoleta, Juperflua: vis : diuretica.

vsvs: Scorbutus,

COMP。

\section{A MYRIS.}

273. AMYRIS Elemifera foliis ternatis quinato-pine natisque fubtus tomentofis. Syft. nat. I2. p. 266. Icicariba. Marcg. braf. 98.

loc. Nova Hifpania.

Arbor, peregrina.

PHARM. ELEM I Refina.

CVAr. pellucida, flavefcenti - fubvirefcens : fumo fuavi.

Rarior.

vis: vulneraria', diuretica.

vsvs: Vulnus capitis, Punctura, Dolores, Tumor. comp. Balf. arcxi. 
374. AMYRIS Opobalfamm foliis pinnatis, folio. lis feflilibus. Amon. acad.7. p. 68.* Mant. pl. 65. Fors/k. flor. 79.

Opoballamum. Alp. cegypt. 48. Geoffr.mat. med. 473 . Dal. pharm. 308.

Loc. Arabia felix.

PHARM. MECHAE, Balfamum Gt. xij.

X Y L O B A LS A M I Lignum.

C $\triangle$ R POB LLS M I Fructus.

QVAl. tenaci-liquida, refinofa. Rarior, preftans. vis: detergens, diuretica! ballamica, antifeptica, vulneraria, emmenagoga, cofmetica.

rsve: Afthma, Phthifis, Gonorihoca.

COMP.

\section{AWSONIA.}

275. LA W SONI A inemis ramis inermibus. F. zeyl. 135. Sp. pl. 498.

Pontaletsce. Rbeed. mal. 4. p. II 7. t.57.

Loc. India, Aegyptus, Syria. Arbor, fera. PHARM. ALCANNAE VERAE Radix, Tintura, Folia.

Cr. Rad. rubra. Fol lutea. Rarior, preftans, Turcica. vis: adflingens, tinctoria.

vsvs: Sophifficatio, Formofitas.

comp. Vngu. rub. potabile, Iincture rubre, Decocta rubra.

VACCI-

274. [Alia ab Amyride diverfa firps, quae Balfamum Mechamitn verum flindere dicitur, a Turca quodam ex agsro Meccenfi allata, opera viror. cll. Keisuegrs, Hacquet et Gibditch nuper innotuir, bALSA IitEA mercanensis Giet. dieta, cui Cal. 5 -partitus. Cor. 5-pet. xqualis, rubra. Stam. 10. longa calyci iuferta Pift. x. fuperum, Ryl. I. Rigm. obut. Fol. bip'nnara: toliolis lanceolatis. Schriften der berliser Gef. zatu:f. Friunde vol. $3 . \mu, 1=7$. Squ. ]

$27^{\circ}$ AL ANNA raro et vix nunquam in nefiris offeinis occurrit, led gus loco et nonine finchasa tinktoria. 


\section{VACCINIVM.}

276. VACCINIVM Myrtillus pedunculis unifloris, foliis lerratis ovatis deciduis, caule angulato. $F l$. Jivec. 333. Sp. pl. 498.

Vitis idra foliis oblongis crenatis, fructu nigrican. te. Baub. pin. 470.

Loc. Europx, prafertim Suecire fylva.

Frutex, vulgatiffmus. Pharm. MYRTILLI BACCAE, Syrupus. QVAL. acidula. Rarior. vis: fubrefrigerans, fubadftringens.

vsis: Diarhoca, Scorbutus.

COMP.

277. VACCIN IVM Vitis idee racemis terminalibus nutantibus, foliis obovatis revolutis integerrimis fubtus punctatis. Fl. flec. 334. Sp. pl.500. Fl. dan. t. 40.

Vitis idxa foliis fubrotundis non crenatis, baccis rubris. Baub. pin. 470.

coc. Europa, prafertim fylvx Suecix.

Frutex, vulgatifinus. PHARM. VITIS IDAEAE Rob.

QVAL. acida.

Preftans:

vis: refrigerans, attenuans.

vsvi: Febrium xtus.

CONP.

\section{DAPHNE.}

278. DAPHNE Meжereum floribus feffilibus ternis caulinis, foliis lanceolatis deciduis. Sp. pl. 504 . Fl. dan. t.268. [Pall. rol]. 1. p. 54.]

$$
\text { II } 4
$$

Lauřco-

s7\%. VITIS IDAEA non recepta eft in nofris officinis, quam et vis ct copia commendat. 
Laureola folio deciduo, flore purpureo. Banb. pin. 462 . Loc. Europæ fylva umbrofx. Frutex, vicur. PHARM. LA VRE OLAE Cortex.

COCCIGNIDII Semina.

Qv. infipida, tum acris, feptica, toxica. Ravior, cauta. vis: corrodens, rubefaciens, excorians, nimulans, pellens, vomitoria, draftica, cardialgica, febrilis. vsvs: Hydrops, V'lcus cacoëthicum, Carcinomas

[Tuflis ferisa, ] Lupibus. comp.

279. DAPHNE Laureola racemis axillaribus quil1quefloris, foliis lanceolatis glabris. Sp. pl. 510. Gesn. fafc. r, p. 7. t. 6. f. 9. Gacq. auftr. 2. t. 18\}. Laureola fempervirens, flore viridi. Bauh. pin. 462. Loc. Italia, Anglia, Germania. Frutex, manfueurs. PHARM. MEZER EI Cortex.

QVAL. acris, feptica, toxica. Exoleta, cauta. vis : corrodens, rubefaciens, excorians, draftica, vsvs: Hydrops, Externus.

COMP.

\section{$T \cdot R I G I N I A$. POL Y GON V M.}

280. POL Y GONVM Biftorta caule fimplicifimo monoftachyo, foliis ovatis in petiolum decurrentibus. Sp. pl. 5 I6. Fl. dan. t. $42 \mathrm{I}$.

Biftorta major, radice magis intorta. Baub. pin. 192. Loc. Germanix prata fubhumida. Perennis, cicur. pharm. BIS TOR T AE Radix. QVAL. Ityptica, rubra.

Vjitata, laudabilis. Vis:

279. MEZEREI cortex, fi hac (279) fpecies Daphnes non occurrat, commode a priore ( 278 ) fpecie defumi potelt. [Cel. Besgin anciore e Dapkne Thymiclan defumendus eft Mat. med. 197. Cl. Leroy ufum corticis Daphnes Guitii commendat in lib. Sur l'écorce do Garou. $P a r, 1 \rightarrow 6 ?$. 
vis: adtringens.

vsvs: Dylenteria, Leucorrhoa, Stomacace, Pro. Hivia.

comp. Diafcordium; Acet. Theriacale.

281 POL Y GONVM Hydropiper floribus hexandris lemidigynis, foliis lanceolatis, ftipulis fubmuticis. Fl. juec.343. Sp. pl. 517.

Perficaria urens feu Hydropiper. Babb. pin. Ior. ıос. Europx noftrx fubhumida. Anmua, vulgatiffma. HARM, YERSICARIAE Herba, $\nabla$.

Q'AL. acris, urens, lutea.

Rarior. YIs: incidens, corrodens, rubefaciens, ftimulans, diuretica.

vsvs: Calculus! Odontalgia, Excoriatura oris, COMP

283. POL YGONVM nviculare floribus octandris trigynis axillaribus, foliis lanceolatis, caule procumbente herbaceo. H. Juec.339. Sp. pl.519. Fl. dan. t. 803 .

Polygonum latifalium. Baub. pin. 28r.

roc. Europx culta, ruderata. Annua, vulgatifima. PHARM. CENT VMNODIAE Herba.

QVAl. lubinfipida, inodora. Exoleta, fuperfun. vsvs: fubadnringens, vulneraria.

vsvs: Prolapfus.

283. POL YGON VM Fagofyrum foliis cordato - fagittatis, caule erectiulculo inemi, feminum angulis xqualibus. 1\%. fuec. 355. Sp.pl. 522.

Fagopyrum. Dod. pempt. 512 .

Lor. Afia. Amma, cerealis, cicur. PHARM. F $\triangle$ GOPYR I Scmina.

QVAL. farinacca.

Culinaris, rarior. 
vis: refrigerans, obftipans, nutriens. vsvs: cibarius.

cOMP.

\section{SAPINDVS.}

284. S A PINDVS Saponaria foliis impari - pinnatis, caule inermi. Sp.pl.526.

Nuciprunifera arbor americana, fructu faponario orbiculato nigro. Pluk.alm.255. t.217.f. 7. Comm. bort. I. p. I83. t. 94 ?

coc. Brafilia, Jamaica. Arbor, fera. PHARM. SAPONARIAE NVCVLAE. QVal. fpumefcens. vis: faponacea.

vsvs: Chlorofis! Leucorrhora.

COMP.

\section{TETRAGYNIA. PARIS.}

285. PARIS quadrifalia. Fl. fuec. 346. Sp. pl. $52 \%$ Fil. dan. 139 .

Solanum quadrifolium bacciferum. Baub. pin. 167. LOC. Europæ umbrofa. Perennis, vulgaris. Pharm. PARIDIS [Herba,] Baccz.

QVAL. tetra, dulcefcens. Preftans. VIS: antifpasmodica.

vsvs: [Convulfiones, Mania, Bubones, ] Pleuritis, Ophthalmia.

COMP. 


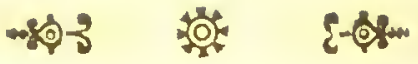 \\ CLASSIS IX.}

\section{E N N E A N D R I A. MONOGINIA.}

\section{LAVRVS.}

286. LAVRVS Cinnamomum foliis trinerviis ovato. oblongis: nervis verfus apicem evanefcentibus. Sp. pl. 528. [Aublet. guian. 362.]

Caflia cinnamomea. Herm. lutgdb. 129. t. 655. Burm. $2: y l \cdot 63 \cdot t \cdot 28$.

ıoc. Zeylona, Martinica. Arbor, peregrina. sharm. CIN N A NOMI Cortex, Syrupus, $\nabla$ fine vino, $\nabla$ cum vino, $\Omega$, $\circ$ o Gitj.

QVAL. dulcis, dein acris, aromatica, fuavifima, fragrantifima.

Trita, beroica. vis : cordialis, analeptica, corroborans, calefaciens, perlpirans, antifebrilis, carminativa, itomachica. vsvs: Debilitas, Vomitus, Tympanitis, Leucorrhæa, Menorrhagia.

comp. Baifan. Cinnamomi, Aqua Cinnamomi hordeata, Aqua Cinnam. cydoniata, Andromach. Mithridat. Diafcordium, Orvictan. Philonium, Diaphœnic. Conf. Alkermes, Hamech, Hiera picra. Balsam. Apoplect, Aqua confortans perlata.

B. Katou-karua. Rbeed. mal. 5. p. ro5. t. 53.

I. co. Malabaria.

IHARM. MA],AHATHRI Folium.

QVid. acris aromatica, fragrans, debilis.

vis: calcfacicns, fimulans.

vsvs: Paralyfis lingux.

comp. Andromach. Mithridat. 


\section{ENNEANDRIA. MONOGYNIA.}

287. LAVRVS Cafia foliis triplinerviis lanceolatis. Fl. $\approx c y l$. 146. Sp. pl.328.

Callia malabarica. Herm. lugdb. 130. Burm. zeyl.63. t. 28 .

roc. Malabaria, Java, Sumatra, Arbor, peregrina. PHARM. CASSIAE LIGNEAE Cortex.

eval. Cimamomi, debilior. Rarior, preftans. vis": Cinnamomi, debilior magisque adfringens. vsvs: Diarrhœa.

comp. Andromach. Mithridat. Philonium, Diafcordium, Troch. Hedycroi, Spec. Diamofchi.

288. LAVRVS Campbora foliis triplinerviis lanceo. lato-ovatis. Sp. pl.528. Thumb. jup. 172.

Laurus camphorifera. Kcempf. amon. 770. t. 77r. Breyn. cent. I6. t. I. Comm. bort. I. t. 95.

Loc. Japonia. Arbor, fera. pharm. CAMPHORAE Refina Grj, -. $\ni \beta, \Omega, \circ$. CVAL. aromatica, acris, amaricans, fragrantiflima, frigida, antifeptica, antifpasmodica. Trita, beroica.

Vis:

286. 287. [Ex mente Cel. Bergii mat. med. 204. J97. et fecundum Pharmacopocasn fuecicams Holm. I579. dat:

A. LAVRVS Cinnamomm n.203.

PHARM. CINNAMOMI VERI Cort.

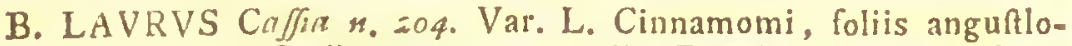
ribus obtufioribus, ex mente ill. Thmnbergii, AR. Holm. I 8 80. $\mu .56$.

Carua. Rlued. Mal. I. p. I07. t.57.

PHARM. CINNAMOMI INDICI Cort.

MALABATHRI Folia.

C. LAVRVS Mlalabathrum foliis trinerviis ovatis acuminatis nervis verfus apicem confluentibus. Burm. iud.92.

Katou karua. R/seed. mal.5. p.105. t.53.

I.oc. India orientalis.

pнакM. CASSIAE IIGNEAE. Cort.]

288. [Aliam arborem camphoriferam, a Lauro Camphora diverfan, adhuc tamen obfcuram, commemorat $\mathrm{Cl}$. Miller in Phil.

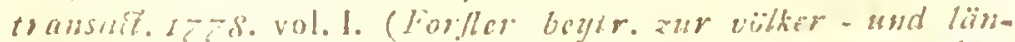
derumde $I . \mu .19$ ) ) eandem, quam Syluius in l'alentini lnd. litt. $1 \% .883$. delcriplit et cujus lolia ibit. 6.7 . delineavit ] 


\section{ENNEANDRIA. MONOGYNIA.}

vis: refolvens, nervina, pellens, fudorifera, an. thelmintica, refrigerans.

vsus: Contagium, Inflammatio, Febres, Mania, Epilepfia, Hyfleria, Odontalgia, Ophthalmia, Rheumatifmus.

comp. Vngu. Album, Pulv. pannonic. Conf. Hyacinth. Vngu. Aegypt. Empl. Stictic.

39. LAVRVS Calilaban foliis triplinerviis oppofitis. Mantifl.pl.237. +

Cortex caryophylloides. Ramph. amb.2. p.65. t.14. E. N. C. lec. 2. ann. 3. p. 53 .

LOC. India orientalis.

PHARM. CVLIL AW A N Cortex, 0.

QVAL, aromatica, mucilaginofa, fubadftringens.

Eximia, rarior.

vis: calefaciens, carminativa! ftomachica, roborans, antiparalytica.

vsvs: Colica flatulenta.

CONP.

30. LAVRVS' nobilis foliis venofis lanceolatis perennantibus, Horibus quadrifidis. Hort. cliff. 155. ups.98. Sp. pl.529.

Laurus vulgaris. Baub. pin. 460.

ı.oc. Græcia, Italia.

внarm. LAVRI Paccæ, $\nabla$, (0), oo, Folia. QVAr. calida, fubacris, aromatica. Tirita, preftans. vis: ftomachica, calefaciens, refolvens, pellens, carminativa, phthiriaca.

vsvs: Colica lochialis.

comp. Eal. baccar. lauri. Empl. baccar. lauri, Orvietan. Diateffaron. Empl. ranar. Limpl. Atictic. Limpl. manus dei.

эr. LA VRVS Saffafras foliis trilobis integrisque. Hort. cliff.154. Sp.pl.530. Trem. ebret.34.t.69.70. 


\section{I2G ENNEANDRIA. MONOGYNIA.}

Cornus mas odorata, folio trifido: margine plano, Sallatras dicta. Catesb. carol. I. p.55 t.55.

ıoc. Penlylvania, Virginia, Florida. Arhor, man/ueta. pharm. SASSAFRIS Lignum, Cortex, Eefentia, $\nabla, \stackrel{n}{V}, \stackrel{\circ}{\circ}$

QVAz. lubdulcis, fubacris, aromatica, fragrans.

$$
\text { Tilta, pricenans. }
$$

vis: diaphoretica, fudorifera, pellens, corrobo. rans, purificans.

vsys: Syphilis, Cachexia, Hydrops, Scabies, Flatulentia, Catarhus, Rheunatimus, Mithritis. CoMp.

292. I $\Lambda$ VRVS Pecurim [qux Lauraner amboinenfis, leytun. Rumpb. amb. 2. p.70.t. 15. an? - Berg. med. 2II.]

Loc. Paraguay.

Arbor, peregrina. PHARM. PECVRIM (PICHVRIM) Faba.

Qval. aromatica, fubftyptica. Tirita, proftans. vis: fubadnringens, flomachica.

visu: Diarliaa, Dyenteria, Tomina. COMP.

\section{A NACARDIVM.}

293. A N A C A R D IV M occidentale. Hort. cliff. 16 r. Fl. zieyl.165. Sp.pl. 543. Gacq. amer. 124.

Kapa-mava. Rbeed. mal.3. p.65. t. 54. Acaju. Pi/. braf. 120.

zoc. Brafilia, Surinama, Malabaria. Arbor, fera. pHARM. ANAC'ARDII OCCIDENTALIS Nux (teftr melligo) [Cortex.] QYAL. melliginis exurens, nigra.

Exoleta.

VIs: excorians, tincloria.

292. [Puxiri Brafilienfibus. Frutus difpermus, Ecyart. ( $\%$ sum\%

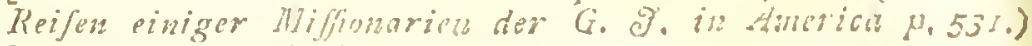
Ergo non gencris Lanti.] 
rsis: Macula, Scabies, Impetigo. [Dyfenteria Cort.] coist

\section{$T R I G I N I A$. \\ RHEVM.}

294. RHEVM Rbaponticun foliis glabris, petiolis lublulcatis. Sp. pl. 53 1.

Rhaponticum thracicum. Alp.rbapont. I. t. 1. exot. 37. ioc. Thracia, Scythia. PHARM. RHAPONTICI Radix 3 j.

QHa. fequentis, flyptico-anara, naufeofa. Exoleta. vis: purgans debilior, adftringens preftantior, fto. machica.

vsvs: Diarrhoa, Leucorrhœa.

cours. Andromach.

295. R HEVM palmatum foliis palmatis acuminatis. Sp. pl.53s. Liznn. fil. fafc. I. p. 7. t. 4. AEt. angl. $1765 \cdot$ p. 292. t. 12 .

Ioc. Mongalia ad confinia Sinarum. Perennis, cicur. PH.AR.M. RHABARBARI VERI Radix 3j.tofta $\Xi_{i j}$. Eefentia 3 j. Extractum 3 j.

Q:Al. fiyptico - amara, naufeola, lutea.

Tritn, beroica, ufitatiffma. vis: purgans, antacida, tonica, adftringens, ftomachica, diuretica, exanthematica, anthelminthica. vsvs: Colica, Rlachitis, Diarrhoca, Lienteria, Dyfenteria, Leucorrhœa, Gonorrhœa, Hectica puerilis, Icterus, Hypochondriafis.

comp. Syr. Cichorei c. Rheo zij. Pulv. cont. vermes, Conf. Hamech, diacath. Extr. Panchymag. Pil. Cathol. fne quibus, Tinct. Anima Rhab. 芌i. CLAS-

295. [Rhetm vel palmatum vel compartum. Ph. rofl. Conf. de Rhabartaro 11l. l'allas it. 3. p. 155. fqq. Cl. Georgi it. x. p.210. $\int 99.7$ 
CLASSIS. X.

D E C A N D R I A.

\section{MONOGYNIA. \\ SOPHORA.}

296. SOPHOR A beptapbylla foliis pinnatis: foliolis feptenis glabris, Sp. pl.533.

Anticholerica. Rumph. amb.4.p.60.t. 22.

LOC. India orientalis.

Frutex, ferus. PHARM. $\Lambda$ N T ICHOLERICAE Radix, Semina. QVAL. amarifima.

Eximia, rarior.

VIS:

vsvs: Cholera! Pleuritica, Colica, Dyfuria.

COMP.

\section{ANAGYRIS.}

297. ANAGYRIS fetida. Clif. bift. I. p.93. Raj. bift. 1722. Sp pl.534.

uoc. Italia, Hifpania. Frutex, manfuetus. pharm. AN A G YR ID IS Folia, Semina.

Cyal. tetra, fotida, amara.

Exoleta.

vis: purgans, pellens, repellens.

vsvs: Abortus, Tumores.

COMP.

\section{HYMENAEA.}

298. HYMEN AEA Courbaril. Hort. ciliff. 484. ups. 305. Sp. pl. 537 .

Ceratia diphyllos antegoana, ricini majoris fructu. Pluk. alm. 96.t. 82. f. .

Loc. Brafilia.

PHARM. ANIME Refina.

Arbor, fera.

RVAl. fubflavefiens, pellucida.

vis : nervina.

Exoleta. 
vsis: Contractura, Paralylis.

cust :

\section{A SSIA.}

299. CASSIA Fiftula foliis quinquejugis ovatis acuminatis glabris, petiolis eglandulatis. Fl. zeyl. 149 . Sp. pl. 540 .

Conna. Rbeed. mal. 2. p. 37. t. 22. Chaiarxambar. Alp. eggpt. I. t. I.

Loc. India orientalis, Egyptus, Arabia, Mexico.

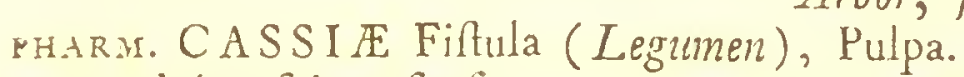
Arbor, fera. QVAL. dulcis fubnaufeola.

vis : demulcens, eccoprotica, febrium purgans.

vsvs: Obftipatio, Nephritis calculofa.

comp. Elect. Diacath. Conf. Hamech.

300. CASSIA Senna foliis fejugis fubovatis, petiolis eglandulatis. Sp.pl. 539 .

a. Senna alexandrina, foliis acutis. Baub. pin. 397. Raj. bift. $17+2$.

PHARM. SENNAE ALEXANDRINAE Folia, Folia f. i. Syrict. Folliculi.

ß. Senna italica 1. foliis obtufis. Bauk. pin. 397. Burm. ind. 96. t. 33. f.2. [Soliva monogr. Madrit 1774. c. tab.]

Luc. in fatis Italix ( $\beta$ ), et India ( $\alpha$ ).

Frutex, fubmanfuetus. PHAR. SENNAE ITALICAE folia 3j. Extractum $习 i \beta$.

QVAL. amara, nauleola. vis: purgans, pellens, repellens.

Trita, eximia. YSY: :

300. [Conf. Caflia lancolata Forsfh. Ror. 85.] 
vsvs: Obftipatio, Herpes.

comp. Extr. Panchymag. El. cathol. Conf. hamech. Decocta.

30r. CASSIA alata foliis octojugis ovali_oblongis: interioribus minoribus, petiolis eglandulatis, ftipulis patulis. Sp.pl.541. Mant. 378. " Facq. obf. 2. p. 24.t. 45.f.2.

Herpetica. Runph. amb. 7. p. 35. t.18.

I.oc. India utraque.

PHARM. HER PET ICAE Folia.

Frutex, ferus.

QVAL. precedentis.

vis : purgans.

vsvs: Herpes. Brown. [Scabies.]

COMP.

\section{MYROXYLON.}

302. MYROXYLON Peruifera. Suppl. pl. 233.

Hoitziloxitl. Hern. mex. 51. c. fig.

Cabureiba. Pif. braf. 57. I19.

цoc. Peru, Mexico, Brafilia. Arbor, peregrina. PHARM. PERVVIANVM Balfamum gr. viij.

QVAL. tenax, flavefcens, amaro-acris, fuaveolens. Trita, preftans.

vis: vulneraria, emmenagoga.

vsvs: Afthma, Phthifis, Vulnera, Vlcera.

COMP.

\section{A ES A L P N I A.}

303. CAESALPINIA brafilienfis caule foliisque inermibus. Sp. pl. 544 .

Pfeudo-fantalum croccum. Catesb. car. 2. t. 5 1.

roc. Janaicre, Carolinx colles. Arbor, peregrina. RHARM. BRASILIENSE LVTEVM Lignum. 
Qval. flava.

Exoleta.

$\mathrm{v}$ is : tinctoria.

vsis : ccononicus.

COMP.

304. CAESALPINIA vefacaria caule aculeato, fo. liolis obcordatis fubrotundis. Sp. pl. $5+5$ ?

Ibira pitanga. Marcgr. braf. 1or. t. 102

soc. Brafilia.

Arbor, peregrina.

PHARM. BRASILIANVM RVBRVM Lignum (medullare).

QVtL. rubra.

Exoleta.

vis: tinctoria.

vsvs: œconomicus, Febres.

cons.

305. CAESAL.PINIA Sappan caule aculeato, foliolis oblongis inxquilateris emarginatis. Fl. zeyl.158.* Lignum Sappan. Rumph. amb.4.p.56.t.2I.

Tfiampangam. Rbeet. mal.6. p.3. t.2.

Loc. Leylona, Malabaria. Arbor, peregrina. PHARM. SAPPAN lignum.

eral. palide fulco-rufa.

Exoleta. vis. tinctoria:

rsvs: œconomicus.

r.MP:

\section{G VILANDINA.}

306. GVILANDINA Moringa inermis, foliis fubbipin. natis: foliolis inferioribus ternatis. Fl. zeyl. ${ }_{555}$ Sp.pl. 546 .

\section{2 Morin-}

305. [SAPPAN Lignum, cum Campefcano non confundendum, hoc magis rubens, e Bengala precipue advelitur.]

306. Behens Nux ro batanus migrillica a quibusdam ex alia fpecie defumitur, urrum vero cingener., adhuc dubiun; hane Hormarnus declaavit. 
Moringa zeylanica, foliorum pinnis pinnatis. Burm. zeyl. 162. t. 75 .

Loc. Zeylona, Malabaria. Arbor, peregrina. pHARM: NEPHRTTICVM Lignum, Tinitura. BEHEN (O).

QYAL: flavo-pallefeens, caruleo tingens, fubacris, amaricans. (O) pinguis, tardifime rancelcens.

Exoleta.

vis: pellens, diuretica. (D) obtundens.

vsvs: Scabies, Exanthemata.

comp. (D) bafis balfamorum.

\section{G V A J A C VM.}

30\%. GVAJACVM offlinale foliolis bijugis obtufis. Sp. pl. $5^{\circ} 6$.

Pruno vel evonymo affinis arbor, folio alato.

Sloan. jam. 2. p. 133. t.222.

roc. Jamaica, Hifpaniola. Arbor, fera. PHARM: LIGNI SANCTI (GVAJACI) Cortex, $\Omega$., $\circ$, Refina, Extractum. [Fructus.] QVar. ficca, amaricans, acriufcula. ITitce, proftanss. vis: aperiens, purificans, pellens, viferalis; Ext.

fternutatoria. [Fruct. emetica.]

vsvs: Syphilis, Leucorrhaa, Arthritis, Podagra, Scabies.

COMP.

\section{DIC'TAMNVS.}

308. DICTAMNVS albus. Hort. cliff. 161. "pS. 102. Sp. pl. 548. Facq. auflr. 5. t. 428 .

Dictammus albus vulgo t. Fraxinella. - Baulb. pin.222. noc. Narbonx, Gemanix colles. Peremins, cicar. PHARM: DICTAMNI ALBI Radix.

Q $\mathrm{V} \wedge$. amara.

Rarior, dubia. vis: 
vis : hal amica, pellens, anthelininthica, emmenagoga. vsrs: Ripileptia, Vermes!

cosp. Orvietanm, 吉guttetx, 专 pannonicus.

\section{R VTA.}

309. R T T A graveolens foliis decompofitis, foribus Iateralibus quadrifidis. Hort. cliff. x45. ups. 103. Sp.pl. 548 .

Ruta hortenlis latifolia. Baub. pin. 336. Dübam. arb. 2. t. 6 1.

1.0\%, Africa, Grecia, Hetruria, Narbonal Frutex, cicur. PHARM, RVTAE HORTENSIS Herba, Conferva, $\nabla$, ( ), $\circ, \theta,+$, Semina.

QYi. acemina, amara, tetra. Trita, beroica. Is: ictolisens, alexiteria, antivenerea, pellens, caiminativa, emmenagoga, exanthemathica, fudorifera, anthelminthica, phthiriaca, rubefaciens. vsio: Peftis, Epilepfia, Hyfteria, Cephalalgia, Oplithalmia, Singultus.

comp. Balfomus Rutx, Acet. prophyl.

\section{TOL, VIFER A.}

310. TOLVIFER A Balfaimum. Sp.pl. 549 .

Palfamum tolutanum foliis ceratix fimilibus. Baub. jin. $40 \mathrm{I}$.

zoc: Provincia Tolu prope Carthagenam Ameri$c x$.

Arbor, peregrina. Qval. fufen-virefcens, dulcis, fragrans, tenax, tandem fragilis.

vis: diuretica! purificans, vulneraria.

vsvs: Phthifis! Punclura, Gonorrhesa, Vlcera,

Vulncra.

COMP.

Trita, preftans. 


\section{HAEMATOXYLON.}

31r. HAEMATOXYLON Campecbiamum. Sp. 11.549.

$$
\text { Facq. obf. I. p. } 20 .
$$

Lignum campechianum. Sloan. bift. 2. p.183.t.X.

f. I-4. Catesb. car. 2. p, 66, t.60.

roc. Campechia Americes.

PHARM. CAMPESC AN V M Lignum.

evar. Ayptica.

Eximis.

vis: adftringens, tinctoria.

vsis. Dyfenteria.

COMP.

\section{SWITENIA.}

312. SITITENIA Mabagoni. Sp. pl.548. Facq. bift. 127.

Arbor foliis pinnatis, fructu angulofo, fem. alato.

Catesh. car. 2. p. 81. t.8I.

Loc. Infula Caribax.

тHARM. M $\Lambda$ HAGON I lignum.

Arbor, peregrina.

CVal. brumneo - fufca.

vis: mechanica.

vsvs: œconomicus. [Cephalalgia.]

COMP.

\section{QV^SSIA.}

313. QVASSIA amara foliis impari - pinnatis: foliclis oppofitis fellilibus, petiolo articulato alato, Hloribus racemolis. Amen.acad.6.t.429. (flores, non folio.) Suppl. pl. 235 .

zoc. Surinama.

Arbor, fera.

PHARM. QV SSSIAE LIgHUM.

QVAL, amariflima, inodora.

Eximia.

313. [Hujus Succedaneum in India occidentali Rhus Mnotorium, etiam Qualfet sonune in Europam millum. D. Concly in lit.] 


\section{1)ECANDRIA. MONOGYNIA. I35}

vis: ballanica, antacida, antiputredinofa, tonica, formachica.

rsw: Febres Intermittentes! Exacerbantes! Hypochundrialis, Arthritis! Leucorrhœa, Sphacelus. c().3!?

314. OYASSTA Simaruba foribus monoicis; folits ab. rupte pinnatis: foliolis altermis fubpetiolatis, peti.lo nudo, Horibus paniculatis. Suppl. 234.

Sinarouba amara. Aubl. gruian. 860. t. 33 I. 332.

r.oc. Guiana Imerices.

Arbor, manfieta.

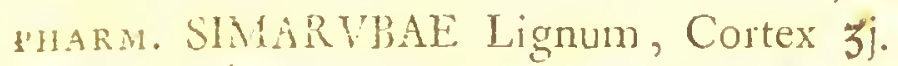

(uvA.. inodora, amaricans. Preftans, wfitato. is: tomica, fubadfringens, diuretica, Atomachica. rsvs: Dyfenteria! Diarhoca, Lienteria.

comp.

\section{LEDVM.}

315. LEDVM paluftre foliis linearibus fubtus hirfutis, thoribus corymbofis. Fl. juec. 352. Sp. pl. 56r. Aincen acad. 8. p. 268.

Cifus ledon foliis rolinarini ferrugineis. Baub. pin. 407.

roc. Suecix Septentrionisquc paludes afperx.

Frutex, vulgaris. YHAR.M. ROSMARINI SYLVESTRIS Herba ziv, lirfulum.

RVAr. amaricans, nidorofa.

Heroïca. vis: narcotica, phantaflica, inebrians, cephalalgica, fuctorifera, exanthematica, emetica, corro. borans, anthelminthica?

vsis: Scabies, 'linea, Lepra! Tuflis ferina, Angina Atrangulatoria, Cephalalgia, Febres cxanthicmatic $x$ ?

cosp. 


\section{RHODODENDR VM.}

316. RHODODENDRVM Cbryfanthum foliis oblongis impunclatis fupra $f$ cabris venofiflimis, corolla rotata irregulari, gemma florifera tomentola. Suppl. pl. 237 .

Rhododendron Chryfanthum. Pall. it. 3. p. 3696 729. tab. N. fig. 1. 2. Fl. rolf. I. p. 44. t. 30. Kalfin monogr.

I.OC. Dauuria.

Frutex, peregrinus. PHARM. RHOD. CHRYSANTHI Folia $z^{i j}$, Infusum. QVAl. amaricans, nidorofa, amaro - auftera. Herö̈ca. vis: narcotica, phantaftica, inebrians, cephalalgica, emetica, purgans.

vsvs: Arthritis! Rheumatifmus, Ifchias, Carcinoma. Syphilis.

\section{ARBVTVS.}

317. ARBVTVS Vva ur $\_$caulibus procumbentibus, folis integerimis. Fl. lapp.162. Juec.358. Sp.'pl. 566. 'Fl. dan. $t .33$.

Vva urfi. Clus. bift. I. p.63.

roc. Suecir colles fabulofi.

Frutex, vulgaris.

PHARM. VVAE VRSI Folia.

QVAL. ftyptica, inodora. Rarior, preftans. vis: adfringens, coriaria, obftipans, diuretica. vsvs: Calculus.

COMP.

\section{PYROLA.}

318. PYROLA rotundifolia faminibus adfcendentibus, piftillo declinato. $F l . j u e c .360 . S p \cdot p l .567 . F i$. dan. t. 110.

Yyrola

317. VVA VRSI non Hbique in officinas Pharmacopoeormm introdueta, ot a coriariis Inco Rhois receptifima; ob vires corroborantes et adfringentes commendanda. 
Pyrola rotundifolia major. Baub. pin. I9r.

Loc. Europa Iylva. Perennis, vulgaris. PHAR. PYR OLAE ROTVNDIFOLIAE Herba.

Cral. Fubftyptica, inodora.

Rarior.

VIs : corroburans, adftringens.

vsis: Vulnus.

COMP.

319. PYROLA umbellata pedunculis fubumbellatis. Fl. fuec. 363. Sp. pl. 567 .

Pyrola futefcens, arbuti flore. Baus. pin. IgI.

Loc. Europx, Afix et Americx feptentrionalis fylvæ. Fruticulus, vulgaris.

PHARM. PYROLAE VMBELLATAE Herba.

Crat. Ayptica.

vis: corroborans, adfringens.

vsvs: Ifchias.

COMP.

320. PYROLA uniflora fcapo unifloro. Fl. Jue6. 364 . Sp. pl. 568. Fl. dan. 3.

Pyrola rotundifolia minor. Baub. pin. 19r.

soc. Europx, Americx borealis fylvx.

Peremis, aulgaris.

PHARM. PYROLAE VNIFLORAE Herba.?

QYAL. Ayptica.

Vis : adfringens.

vsve: Ophthalmia.

SOMP."

\section{STYR $A$ X.}

321. STYRAX officinalis. Hort. cliff. 1337. upf. 123. Sy/t. nat. 301. Mill. ic. t. 260.

Styrax folio mali cotonei. Banb. pin. $45^{2}$.

ı. Or. Malia, Creta, Syria, Palxflina. Arbor, manefueta. 
mHAn. STORACIS CALAMIITAE Reina, OO. UAL. relinola, fubacris, fragrans. Vjitata. is : nervina, fubanodyna.

vsvs: Vlcera interna, Catarrluus, 'Tullis.

cosp. Pil. Ayracis, Empl. Ayracis, Empl. Aomachale, Andromachi, Mithridat. Diafcord. Balj. apoplcel. Tongu. martiatum. Pulv. fumal.

\section{COPAIFERA.}

322. COPAIFERA officinalis. Sp.pl.557. Gacq. umer. 153. $t .86$.

Copaiba. Marcgr. braf. 130. Pif. braf.56. Barr. cequin. 40.

1.oc. Brafilia, Infula Maranhon, Arbor, peregrina. YHisM. COFAIVAE Balamum $\theta$ j.

eyal. liquido-tenax, Havefcens, acris, amara, alomatica, fragrans, urinaria: urina annara.

Trita, preftans.

vis: vulncraria, diuretica, laxans, mundificans.

rovs: Syrnocha, Phthifis, Heclica, Tufis, Gonor. rhar, Leucorrhara, Diairhcea, Scorbutus, Circumcilio.

Co.MI'.

\section{$D I G I N I A$. SAXIFRAGA.}

323. SAXIFR $A G A$ gramulata foliis caulinis reniformibus lobatis, caule ramofo, radice granulata. Fi. fuec. 372. Sp. pl. 57 6. Fi. dan. t. 514.

Saxifraga rotundifolia alba. Baub. pin. 309.

ı.oc. Europix colles prataque. Perennis, vulgatifina. fHarm. SAXIFragaE ALbaE Radix, Herba, Flores, Semina.

ए广al. acris, pungens. Exoleta, infida, Juperflua. 
ris: diuretica.

vsrs: Calculus?

cOMP.

\section{GYPSOPHILA.}

324. GYPSOPHILA Struthizm foliis linearibus carne. fis: axillaribus confertis teretibus. $S p . p l .582$.

Saponaria lychnidis folio, Hofculis albis. Baub. pin. 206.

Loc. Hilpania.

YHARM. STRVTHII Radix.

eral. Faponacea.

Perernis, manfueta.

VIs: mundificans.

Inufitata.

vsvs: Calculus: (Saponis loco Veteribus, et hodie Hilpanis.)

eOMP.

\section{SAPONARIA.}

325. SAPONARIA officinalis calycibus cylindricis, foliis ovato-lanceolatis. Hort. cliff. 165. unf. 160.

Sp. 116.584. Fil. dan. t.543.

Saponaria major lavis. Baub. pin. 206.

цoc. Germania, Anglia, Gallia. Peremis, cicur. PIJARM. SAPONARIAE Radix, Herba, Semina. CyAl. amara, trita fpumefeens.

Exoleta.

vis: pellens, fudoritera, diuretica, cmmenagoga, anthelminthica.

vsvs: Cachexia, Leucorrhexa, IClerus, Afcarides. $60.1 P$.

\section{DIANTHVS.}

326. DIANTHVS Caryopbyllus thribus folitaris, fquamis calycinis fubovatis breviflimis, corrillis crenatis. Hort. cliff. 164. up). 104. Sp. pl.587. 
Caryophyllus hortenfis fimplex, flore majore. Baub. pin. 208.

xoc. Europx auftralioris Alpes.' Perennis, cicur. rharm. TVNICAE Flores (corolla), Conferva, Syrupus, $\nabla,+$.

QVAL. fragrans.

vis : cordialis, fudorifera, alexiteria.

vsvs: Contagium, Exanthemata, Febres.

comp. Spir. Embryon. Potiones cordiales.

\section{PENTAGYNIA. SED VM.}

327. SEDVM Telepbium foliis planiufculis ferratis,'corymbo foliofo, caule erecto. Fl. fusec. 400. Sp. pl. 6 I6.

Anacampleros, vulgo Faba craffa. Baub.bift.3.p. 68I. roc. Europx colles faxof.' Perennis, vulgaris. PHARM. TELEPHII Radix.

Qval. glutinola, fubacida, mitis. Exoleta, infida. vis: refrigerans.

vsvs: Varix ani, Paronychia, Hxmorrhois? Scor. butus.

COMP.

328. SEDVM acre foliis fubovatis adnato-fefilibus gibbis erectiufculis alternis, cyma trifida. Fl. Juec. 403. Sp. pl. 619.

Sempervivum minus vermiculatum acre. Baub. pin. 283.

Loc. Europx montes, tecta. Perennis, vulgaris. PHARE. SEDI MINORIS Herba, $\nabla$.

uYAL. acris, fubcorrofiva.

Preftans. VIS :

32й. [In Pharm. Aufr. SEDVM MINVS eft Sedusis abum, SEDVM VERMICVLARE autem Sedum acre, et SEDVM

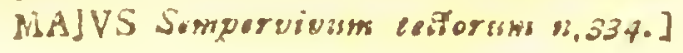


vis: incidens, vomitoria, diuretica.

vsvs: Quartana! Scorbutus! Hydrops!

COMP.

\section{OXALIS.}

329. OXALIS Acetofella fcapo unifloro, foliis ternatis, radice dentata. Fl. Suec. 406. Sp. pl.620.

Trifolium acetolum vulgare. Baub. pin. 330.

Loc. Europx fylve acerofi. Peremis, villgatifina. pHarr. ACETOSELLAE Herba, Conferva, Sym. pus, Sal eflentiale.

CYAL. acida.

Vjitata.

VIs: refrigerans, attenuans.

vsvs: Febres Putrida, Plologifticx.

comp. Fulep. antifebril.

\section{$D E C A G Y N I A$. PHYTOLACCA.}

330. PHYTOLACCA decandra floribus decandris decagynis. Hort. cliff. 177. Hort. ups. II7. Sp. pl. 63x. Solanum racemolum americanum. Pluk. alm. 353. to 225. $f \cdot 3$.

Ioc. Virginia.

Perennis, manfueta. PHARM. HHYTOLACCAE Herba recens, Succus. QY. tenelle aquofa, oleracea, adultce acris. Exim. rarior. vis: corrudens.

vsvs: Carcinoma!

cons. 
CLASSIS XT.

D O D E C A N D R I A. MONOGINIA.

\section{ASARVM.}

331. ASARVM curotcum foliis reniformibus obtufis binis. Fl. Juec. 424. Sp. pl.633. Fl. dan. t. 63j. Afarum. Baulb. pin. 197. i.oc. Europx anflralior.

Perennis, cicur. PHARM. ASARI Radix 3j, Extractum Эj; Folia no.VT. QVAl. amaro-acris, naufeofa. Trita, beroica. vis: vomitoria! purgans! diuretica, diaphoretica, emmenagoga, errlina.

vsus: Melancholia, Coryza. GOMP.

\section{ANEL.LA.}

332. CANELLA alba. Murr. Syft. veg. 14. p. 443.

Canella foliis oblongis obtufis nitidis, racemis terminalibus. Brown. jam. 215. t. 27.f.3.

Loc. Jamaica, Carolina. Arbor, fubrnanfueta. PHARM. CANELLAE ALBiLE Cortex.

Qvat. acris, pungens, fubamara, aromatica, fragrans.

vis: calefaciens, pellens, carminativa.

vevs: Catarrhus, Hæmorrhagia! Paralyfis lingux. comp.

\section{PORTVLACA.}

333. PORTVLACA oleracea foliis cuneiformibus, floribus feffilibus. Roy. bugdb. 473. Hort. upf. 146 . Sp.pl. 638. 
Portuiaca latifolia f. fativa. Baub. pin. 288.

ıoc. Germania, Malabaria, Jamaica. Amma, cirur. pharm. PORTVLACAE Herba, Conferva, Symmpuss, $\nabla$.

crac. aquofa. Culinaris, exoleta, fuperfua. vis: diluens, humectans, iefrigerans, corroborans, lac coaguians.

rsve: Naufea, Scorbutus, Stranguria, Calculus. COMP.

\section{YTHR VM.}

334. LYTHRVM Salicaria foliis oppofitis ternisque cordato-lanceolatis, floribus fpicatis dodecandris. Fl. Juec. 422. Sp. pl. 640. Fl. dan. 67r.

1. yimachia ficata purpurea. Bauh. pim. 246 . Loc. Europa ad aquas. Perensis, vulgaris. PHARM. SALICARIAE (LYSIMACHIAE PVRP.) Herba.

QVAL. mucilaginofa, fubfyptica.

vis: lubadnringens.

Praftans. vis: Diarrhaa, Dyfenteria.

CONP.

\section{$D I G Y N I A$. $\triangle$ GRIMONIA.}

335. AGRIMONIA Eupatoria foliis caulinis pinnatis: impari petiolato, fructibus hifpidis. Fl. fuec. 423. Sp.pl. 642. Fl.dan. t. 588.

Eupatorium veterum f. Agrimonia. Baub. pin.341. roc. Europe prata. Perennis, vulgaris. PHAR.M. AGRIMONIAE Herba, $\nabla$.

QYAL. Atyptica.

Triti. vis: adftringens, roborans, pellens, diuretica, vulneraria.:

comp. 


\section{$T R I G, Y N I A$. EVPHORBIA.}

336. EVPHORBIA officinarum aculeata nuda multan. gularis, aculeis geminatis. Hort. cliff. 106. ups. 138. Sp. pl.647.

Euphorbium cerei effigie, caulibus craffioribus, fpinis validioribus armatum. Comm. hort. I. p.2r. t. Ir. roc. Africa.

PHARM. EVPHORBII Gummi, (O).

Cral. lactefcens, acris, feptica. Rarior, cauta. vIs: corrodens, rubefaciens, veficans, exulcerans, ftimulans, pellens, draftica, fternutatoria.

rsvs: Hydrops, Caries, Punctura.

COMP.

337. EVPHORBIA Latbyris umbella quadrifida: dichotoma, foliis oppofitis integerrimis. Sp.pl.655. Lathyris major. Baub. pin. 293.

ıoc. Gallia.

Biennis, cicur. PHARM. CATAPVTIAE MINORIS Semina. QV. Lacteficens, acris, feptica. Exoleta, cauta, fuperfura. vis: corrodens, draftica.

vsvs: Verruca.

COMP.

338. EVPHORBIA paluftris umbella multifida: fubtrifida: bifida, involuccillis ovatis, foliis lanceolatis, ramis fterilibus. Fl. fuec. 427. Sp. pl. 662. Fl.dan. t. $\$ 66$.

Tithymalus paluftris fruticofus. Bauh. piqt. 292. z.oc. Europa.

Perennis, cicur. PHAR.M.

338. Parum refert, e quanam Exphorbiarum in Europa vulgarium Efuler Rad. Cort. Herba fumantur. Euphorbire palufuris loco L. heliofcopiam vel aliam quamcamque colligi poff*, monet Col. Berging matab. Hiod. 248.] 


\section{DODECANDRIA. DODECAGYNIA, I45}

PHARir. ESVLAE Radix; Cortex (radicis), Herba. Qv. Lactefcens, acris, feptica. Rarior, cauta, Hb. Juperfl. vis: corrodens; rubefaciens, veficans, draftica vsve: Hydrops, Odontalgia, Serpigo, Clavus. comp. Eartr. phlegmagog. Quercet. Pil. fort. maj.

\section{DODECAG YNIA. SEMPER VIVVM.}

339. SEMPERVIVVM teetorum foliis ciliatis, propaginibus patentibus: Fl. Juec: 428. Sp. pl. 664. Fl. dan. t. 6or.

Sedum majus vulgare: Baub: pin. 283.

zoc. Europx noftrx tecta: Perennis, infrequens. RHARM. SEMPERVIVI MAJORIS Herba.

QVAL. acido-auftera, aquofa, acriufcula. Rarios: ris : refrigerans; adfringens.

rsvs: Febres, Dyfenteria; Aphtha, Combuftura;

Clavus.

- cospr. 


\section{6

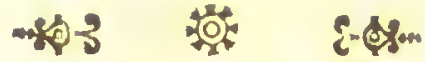 CLASSIS XII. \\ I C O S A N D R I A. MONOGYNIA. \\ MYRTVS.}

340. MYRTVS commmis floribus folitariis, involucro diphyllo. Hort. cliff. 42. ups. 123. Sp.pl.673. Myrtus communis italica. Baub. pin. 468.

loc. Orbis 4 partes temperata. Arbor, manjuta. pharm. MYRTI Folia, Bacca, (O).

QVAl. fubltyptica, aromatica, fragrans. Rarior. vis: calefaciens, corroborans, adftringens.

vsvs: Protluvia.

COMP.

341. MYRTVS caryophyllata pedunculis trifido-multi. floris, foliis obovatis. Sp.pl.675. facq. obf.2.p.r. Caryophyllus aromaticus ind. occid. foliis et fructu rotundis, dipyrene. Pluk. pbyt. 155. $f \cdot 3$.

uoc. Zeylona, Cuba, Guiana. Arbor, peregrima. pharm. CASSIAE CARYOPHYLlat TAE Cortex. QVAL. aromatica, fragrans, pungens.

vis: cephalica, ftomachica, tonica.

vsvs:

COMP.

342. MYRTVS Pimenta foliis alternis. Fl. zeyl. 186. Sp. pl. 676 .

Myrtus arborea aromatica, foliis laurinis latioribus et fubrotundis. Sloan. jam.2. t. 191. $f$. I. Pluk. phyt. 155. f. 4 .

LOC. Jamaica.

Arbor, peregrina. PHARM. PIMENTAE (AMOMI VERI faljo) Fructus. 
CVAL. aromatica, fragrans.

Rarior.

vis : calefaciens, ftomachica.

vsTs: diateticus.

Comp.

\section{PVNICA.}

343. PVNICA Granatum foliis lanceolatis, caule ar. boreo. Hort. cliff. 184. ups. 123. Sp. pl. 676.

a. Malus punica fativa. Baub. pin. 438.

B. Punica Hore pleno majore. Tournef. in itit. 636 .

Loc. Africa, Hifpania, Italia. Arbor, manfueta, Jterilis. pHARM. BALAVSTIORVM $(\beta)$ Flores.

MALICORII Cortex.

GRANATORVM Semina, Syrupus.

QVAL. Ayptica, rubra.

Trita, preeftans.

vis: adfringens, laxans, anodyna $F$., pellens,

diuretica, anthelminthica.

vsvs: Cephalalgia, Prolapfus.

come. Troch. carabe, 宩 dyfentericus, Gargarifmata.

\section{A MYGDALVS.}

344. AMYGDAl,VS Perfica foliorum ferraturis omnibus acutis, Horibus fefilibus folitariis. Hort. cliff. 186. up. 123. Sp. pl. 676.

Perfica molli carne et vulgaris. Baub. pin. 440. zoc. Perfía.

Arbor, manfueta.

PHArm. PERSICAE Flores, Syrupus, Nuclei, $\nabla$.

QVAL. Amygdalix amaræ 345. $\beta$.

Exoleta.

vis: Amygdalæ amarx.

vsvs: Vermes.

conp.

345. AMYGDALVS communis foliis ferraturis infmis glandulofis, floribus fellilibus geminis. Hort. cliff: x86. u乡. เ23. Sp. pl. 677 . 


\section{I4S ICOSANDRIA. MONOGYNIA}

a.Amygdalus dulcis, putamine molliore. Toumef. inft. 627.

s.oc. Tripolis fepes.

Arbor, manfueta.

pharm. AMYGDALAE DVLCIS Nuclei, oblonSi, (O)

QYal. lubpinguis, dulcis, fapida. Trita, prefans. vis: nutriens, obtunkens, emolliens, relaxans. revs: Dolores, Phlogoles, Febres, Tuflis, Colica. COMP.

B. Amygdalus amara. Tournef. inft. 627.

roc. 'Tripolis. Arbor, manfuston PHARM. AMYGDALAE AMARAE Nuclei, (D). QVAL. amara.

Prefteuns, dubia. vis: pracedentis $(\alpha)$ et anodyna, caminativa, pellens, diuretica, anthelminthica.

vsvs: Cophofis, Colica, Ebrietas, Efchara, Vermes, Calculus.

$\operatorname{COMP}$

\section{PR VNVS.}

346. PRVNVS Laurocerafus floribus racemofis, fohis fempervirentibus dorfo biglandulofis. Sp. pl. 679 . Laurocerafus. Cam. bort. 23. Cluf. bift. 1. p. 4.

Ioc. 'Trapezus. Arbor, manfueta. PHARM. LAVROCERASI Folia.

QVAL. amara.

vis: diuretica, narcotica, fere amygdala, amai:x, antifpasmodica, venenata.

vsvs: Phthifis, Syphilis.

COMP.

347. PRY

346. LAVR OCERASVS nondum intravit officinas neftrar.

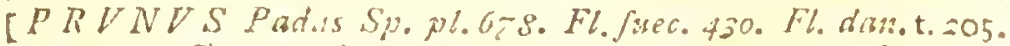
nuper in officinas pharmacenticas Suecicas recepta eft. Cort. Decod. utile in Syphibitide. Ciad. Diürnhtsnd.] 
44. PRVNVS Cerafus umbellis fubpedunculatis, folis ovato-lanceolatis glabris conduplicatis. $S p \cdot p l .679$. Ceralus fructu acido ferotino, fucci fanguinei. Tournef. inft. 625.

r.oc. hodie Europe horti. Arbor", frequens. PiAn. CERASORVM RVBORVM Fructus, Rob, Condita, Nuclei, Gummi.

"YA. aquola acidiulcula, Fapida. Culinaris, uftata. is : refrigerans.

vivs: Febres.

COMPe

3.4. PRVNVS avium umbellis fefflibus, foliis ovatolanceolatis lubtus pubelcentibus conduplicatis. $F$. fuec. 429. Sp. pl. 680.

Cerafus major fylveftris, fructu fubdulci nigro co. lore inficiente. Baub. pin. 450 .

roc. hodie Europe horti. Arbor, frequens. PHARM. CLRASORVM NIGRORVM Fructus, $S y$ -

QVAL. acida, fapida.

$$
\text { rupus, } \nabla, \Omega, \widetilde{V} \text {. }
$$

vis: nervina, antifpafmodiea, fubanodyna.

Vitata.

vsvs: Paralyfis, Convulfiones.

comp. Aqua confortans perlata.

349. PRVNVS domeflica pedunculis fubfolitariis, $f(0=$ liis lanceolato-ovatis convolutis, ramis muticis. Fl. Juec. 430. Sp. pl. 680.

a. Prumus fructu prarvo dulci atro - caruleo. Toumef. inft. 622.

Loc. Furopa aufralis. Arbor, cicur, frequens. yHARM. PRVNA GAILICA, minora, majora, Pulpa. B. Prunus fructu magno dulci atio-cerulco. Toumel. inft. 622 .

LOC. Sytia.

Arbor, cicur. 
IHARM. PRVNA DAMASCENA, Pulpa.

$\gamma$. Prunus brignonienfis, fructu fuavilimo. Tournef. infl. 622 .

Loc. hodie Galloprovincia. Arbor, cicur. PHARM. PRVNEOLAE Fructus (demto nucleo.)

QYA1. acidiufcula.

Culinaris, ufitata.

VIs: refrigerans, humetans, lubricans, eccoprotica. vsrs: liebres, obntipatio alvi.

comp. Pruna laxativa $\overline{\mathbf{j}}$ j.

350. PRVNVS fpinofa pedunculis folitariis, foliis lanceolatis glabris, ramis fpinofis. Fl. fuec. 432. Sp.pl.Gsr. Prunus fylventis. Baub. pin. 444. Loc. Europx colles.

Frutex, vulgaris. rHARM. ACACIAE N'OSTRATIS Cortex, Flores, Symupus, $\nabla$, Fructus, Succus.

QVAL. Aypicica.

Trita, preflans. vis: Rnd. Cort. adnringens, coriaria, Fl. laxans, liv. refrigerans.

vsvs: Profluvia, Diarrhoa, Calculus!

COMP.

\section{$D I G I N I A$. CR A T AEGVS.}

351, CRATAEGVS Aria foliis ovatis incifis ferratis fubtus tomentofis. Fl. /uec. 433. Sp. pl.681. Fl. dian t. 302 .

Alni effigie lanato folio major. Baub. pin. $45^{2}$. Loc. Europa montofa. Arbor, cicur. PHARM. SORBI DOMESTICAE BaccX. eval. farinola, fuccolo-fubfatua. Exoleta, infida. ris:

351. [Has baccas ab ipfa Sorbo domefica Sp. pl. 684. petendas elle monet Cel. Biergius inat. med.264.] 
vis: flatulenta.

viss:

COMP.

\section{TRIGINIA. SORBVS.}

352. SORBVS aucuparia foliis pinnatis utrinque gla: bris. Fl. fuec. 400. Sp.pl.683. [Pall.rolf. I. p. 28.] Sorbus fylveftris, foliis domenticæ fumilis. Baub. pin. $\$ 15$.

r.nc. Euronx fylve. Arbor, frequens. PHARM. SORBI AVCVPARIAE BaccX.

QVAL. acida, farino $\boldsymbol{I}_{\text {. }}$

Exoleta.

vis: adtringens.

vers: llamorthois.

COMP.

\section{$P E N T A G Y N I A$. MESPILVS.}

353. MESPILVS germanica inermis, foliis lanceolatis fubtus tomentofis, Horibus folitariis feffilibus. Hort. cliff. 189. ups. r29. Sp.pl.684. [Pall. rolf. r. p. 20. t. I3. f.I.]

Melpilus germanica, folio laurino non ferrato. Baub. pin. 453 .

ros. Germania et Europa aufralior. Arbor, manfueta. phars. Mespil fructus, Semina.

zуA.. Ayptico-farinofa, eliculenta. Rarior. vis: aditringens, refrigerans.

vsvs: Diarrhca.

COMP. 


\section{PYR VS.}

354. PYRVS Malus foliis ferratis, umbellis fefilibus. Hort. clift. 189. ups. 130. Sp. pl.686.

1.C. Germania.

Arbor, cicur. PHARM. POMORVM BORSDORPHIENSIVM SUEcus liquidus.

eval. acidula, efculenta, fapida.

Parcior. vis: refrigerans, tonica, fubeccoprotica, vsvs: Febres.

comp. Confect. alkermes.

355. PYRVS Cydonia foliis integerrimis, floribus ' $f$ litariis. Hort. cliff. 160. Sp.pl.687. Facq. austr. 4. t.342. [Rall. rolf, I. p.21.]

Cotonea malus. Bauh. bifl. 1. p.35.

Loc. Danubius.

Arbor, fabcicur.

Pharm. CYDONIAE Fructus, Condita, Rob, Syrupus, Succus, Miva, Panis, Cartex, Semina, (0). eval. acido-Atyptica. Sem. mucilaginofa.

Irita, preftans.

vis: adflingens, refrigerans. Sem. invilicans.

vsvis: Naufea, Vomitus, Colica, Cholera, Diar. rhæa, Abortus.

comp. $\nabla$ cinnamomi cydoniata.

\section{SPIRAEA.}

356. SPIRAEA Filipendula foliis interrupte pinnatis : foliolis lineari- lanceolatis ferratis glaborimis, Aoribus cymolis. Suppr.262. Fl. fuec. 439. Sp. pl. Toz. Fl. dan. \$. 635. [Pall. roff. 1. p.39.]

Filipendula vulgaris. Baub. pin? 163 .

coc. Europx prata. Perennis, vengatiliana. PHARM. SAXIPRAGAE RVBRAE Radix, Herba. RVAz.ftypitica. Exoleba, Jingorizua. 
vis: adfringens, vulneraria, lithonthriptica? vsvs: Leucorrheea, Dy-fenteria, Hernia. comp.

357. SPIRAEA Vlmavia foliis interrupte pinnatis: fon liolis ovatis biferratis fubtus canis, floribus, c:mofis. Suppl:262. Fl. fuec. 440. Sp. pl. 702. Fl. dan. t.547. [Pall. rolf. I. p. 40.]

Barba capre lloribus compactis. Baub. pin.164. ıoc. Europx pafcua humida. Perennis, oulgatiffma. PHARM. VLMARIAE Radix, Herba, $\nabla$.

CYA. Atyptica, fragrans. Exolete, preftans. Vis: adfringens, fudorifera.

vsvs: Exanthemata, Dyfenteria, Hernia.

comp. Empl. ruptur.

\section{POLYGYNIA. $\mathrm{R} O S \mathrm{~A}$.}

358. ROSA centifolia germinibus ovatis pedunculisque hifpidis, caule hifpido aculeato, petiolis inermibus. Sp.pi. 704 :

Rola rubra multiplex. Baub. pin. 481. Hort. ups. I32. B.

Loc. Europa.

Frutex, cicur. YHARM. ROSAE RVBRAE Flores, Condita, ConJerva, $\nabla, \circ$, $t$, Succus, Placenta, $\mathrm{K}, \Omega$, $\checkmark$, Antherx, Semina.

Qval. fragrans, rubra, fpirituofa. Trita, preftenis. vis: corroborans, adfringens.

vsvs: Leucorrbeca, Profluvia.

comp. Conferva rofarum vitriolata, Saccb. rofatum, Syr. rofarum ficcarum, Fl. IV cordiales, Aque IV cordiales. 


\section{I54 ICOSANDRIA. POLYGYNIA.}

359. ROSA damafcena germinibus ovatis pedunculis que hifpidis, caule aculeato, calycibus pinnati. fidis hirfutis. Mill. dict. [germ.3. p.854.]

Rofa purpurea. Bauls. pin. 48I.

- Loc. Europa.

Frutex, cicur.

rharm. ROSAE DAMASCENAE Flores, (),

Conserva, recens, antiqua.

cval. fragrans, purpurea, fpicituofa. Tritu, preftans. vis: analeptica, tonica.

VSVS:

COMP.

360. ROSA canina germinibus ovatis pedunculisque glabris, caule petiolisque aculeatis. Sp. pl.704. Fl: dan. t. 555 .

Rofa fylveftris vulgaris, flore odorato incarnato. Baub. fin. 483 .

roc. Europx fylva. Frutex, vulgaris. PHAR ROSAE SYLVESTRIS Flores, Aqua, pallidarum Conerva.

CYNOSBATI Fructus, Semina.

BEDEGrAR Fungus (nidus infecti).

Qval. fragrans, pallida, fpirituofa. Trita, preftans. vis : eccoprotica. Bedegu. adftringens.

vovs: Soda Cynosb.

comp. Syrupus rofarum folutivus $\xi^{j}$. Mel rofarum.

36r. ROSA alba germinibus ovatis glabris, pedunculis hifpidis, caule petiolisque aculeatis. Sp. pl. 705 .

Rola alba vulgaris major. Bauh. pin. 482. Hort. ups. I32. $\delta$.

Loc. Europa.

Frutex, cicur. pharm. ROSAE ALBAE Flores, Condita, Conser$v a, \nabla$.

CYAL.

359. [ROSAS DAMASCENAS Offic. a Rofic centifolia, RVBRAS a galica peterc jubet Finarin. Suec. 28.J 
eval. fragrans, alba, fpirituofa. Trita, praftans. vis: analeptica.

rsvs: Ophthalmia, Leucorrhœa.

coџp: Fulep. roíarum.

\section{R VBVS.}

363. RVBVS idcus foliis quinato-pinnatis ternatisq!e, caule aculeato, petiolis canaliculatis. Fl. juec. 446. Sp. pl. 706. Fl. dan. t. 788 .

Rubus idrus fpinofus. Baub. pin. 479.

Loc. Europx colles faxof. Frutex, vulgaris. PHARM. RVBI IDAEI (Fructuum) Syrupus, $\nabla$, $+, \Omega, \tilde{V}$.

CYAL. acidula, grata.

Trita, preftans.

vis: refrigerans, analeptica.

vsis: Sitis, Febrium xitus.

COMP.

363. RVBVS arcticus foliis ternatis, caule inermi unifloro. Fl. Juec. 448. Sp. pl. 708. Fl. dan.t. 488. Rubus caule unifloro, foliis ternatis. Fl. lapp. 207. t. 5. f. 2 .

roc. Norlandix palcua. Perennis, cicur. PHARM. NORLANDICARVM BACCARVM Condita.

QYAL. acidula, fragrans.

Rarior, eximia. vis: humectans! refrigerans! cordialis! analeptica! vivs: Febres putridx, exanthematicx, Scorbutus! COMP.

364. RVPVS Chamemorus foliis fimplicibus lobatis, caule irermi uniltoro. Fl. Juec. 449. Sp.pl.708. Fl. dan. iab. $\mathrm{x}$.

Rubus caule bifolio unifloro, foliir fimplicibus. F. lapp. 208. t.5. f. 1 . 
$\$ 56$ ICOSANDRIA. POLYGYNIA.

roc. Suecia, Borufix paludes fylvaticx fplizgnofa.

rHARM. CHAMAEMORI Comizta.

Perennis, frequens.

QyAL. aquofa, inodora, efculenta. Rarior, prefanis. vrs: diluens, humectans, refrigerans.

rsvs: Hamopty fis, Phthilis, scorbutus.

COMY

\section{FRAGARIA.}

365. FRAGARIA vefca flagellis reptantibus. Fi. Jree. 450. Sp. pl.708. Aman. acad.8.p. Iбog.

Fragaria rulgaris. Baub. pin. 326.

I.oc. Europac fylva. Perennis, vulgatiffma. IHARM. FRAGARIAE Herba, (Fractulum) Syrupus, $\nabla,+, \Omega$.

cyal. aquofa, acida, fragrans. Trita, preftains. $\checkmark$ is: diluens, humectans, fubrefrigerans, diapliore. tica, diuretica, eccoprotica.

vsvs: Febres, Calculus, Podagra! Phthifis,

CONP.

\section{POTENTILLA:}

366. POTEN"TILLA Anferina foliis pinnatis ferratis: caule repente, pedunculis unifloris. Fl. Juec. 452. Sp.pl. .710. Fi. dan. t. 544.

Potentilla. Bauh. pin.321.

s ос. Europa pafcua. Perennis, sulgatifima. PHARM. ANSERINAE Radix, Herba.

eral. infipida.

Debilis, exoieta.

vis: adtingens.

rsis: Kicrus? Prolapfus, Leucorthoca, Calculis. cosil. 
$36 \%$ POTENTILLA reptans foliis quinatis, caule re. pente, pedunculis unitloris. Fl. fitec. $456 . S p$ pl. 754.

Quinquefolium majus repens. Baub. pin. 325.

koc. Europe ruderata. Peremis, fulgaris: PHARM. PENTAPHYLLI Radix, Herba.

$R+A L$. Aytptica.

vis: vulneraria, adfringens.

Rarior, Hb. Superfuta.

rsis: Dyfenteria.

cous. Gargarifmata, Andromach;

\section{TORMENTILLA.}

368. TORMENTILLA erecta caule erectiufculo, foliis fethilibus. Fl. fuec. 420. Sp.pl.716. Fl. dam. t. 589 .

Tormentilla fylveftris. Baub. pin. 326.

soc. Furopx pafcua. Perennis, vulgatifima. PHA.r. TORMENTILLAE Radix, EJentia, Ex. tractum, Herba.

QYal. Ayptica, rubra. Trita, proftans. vis: adfringens! corraria, diuretica.

yovs: Diarrhoea, Dyfenteria, Stomacace, Hæúaturia?

co.IP.

\section{GEVM.}

359. GEVM urbanum floribus ercetis, fructibus glo. bofis villofis: ariftis uncinatis nudis, foliis ly. ratis. Fl. Suec. 460 . Sp. pl.716. Fil. dan. t.672. [Bucbbave aat. med. bavn.r. p.irg. t. r.]

Caryophyllata vulgaris. Baub. pin.321.

coc. Europa nemora. Perennis, vulgaris. PHARM. CARYOPHYLLATAE Radix, Herba. QVAL. flyptica, fragrans, antacida. Rarior. 
I58 ICOSANDRIA. POLYGYNIA:

vis: adfringens, pellens, fudorifera.

vsvs: Variola! Dyfenteria, Febres intermittentes.

COMP :

370. GEVM rivale floribus nutantibus, fructu oblongo: ariftis plumofis tortis. Hort. cliff. 195. Fl. fuec. 461. Sp tl. 717. Fl. dan.722.

Caryophyllata aquatica, nutante flore. Bauh. pin. 321.

Loc. Europx, prefertim feptentrionalioris, prata humida, uliginola. pharm. GEI PALVSTRIS Radix. QYAL. Atyptica, antacida. vis: adfringens, coriaria.

Perennis, eulgaris. vsvs: Febres intermittentes.

COMP.

Rarior, proftans. 


\section{Sus CLASSIS XIII. \\ P O L Y A N D R I A. $M O N O G Y N I A$. CAPPARIS.}

37r. CAPPARIS fpinofa pedunculis unifloris folitariis, ftipulis fpinofis, foliis annuis, capfulis ovalibus. Sp.pl. 720 .

Capparis finofa, fructu minore, folio rotundo. Baulb. pin. 480 .

Loc. Italix, Narbonx muri. Arbor, fera. Pharu. CAPPARIDIS (Radicis) Cortex, Flores (aceto) conditi.

Cyal. ficca, lubamara.

vis: adftringens, diuretica, emmenagoga.

Vfitata. vsvs: Hypochondriafis, Obftipatio, Paralyfis. comp. Irocb. capparidis, Ol. capparidis, Radices $\mathrm{V}$. aperientes minores, Infula.

\section{A C TAEA.}

372. ACTAEA ramofa racemis longillimis, fructibus ficcis. Sp. pl. 722.

Chriftophoriana americana procerior et longius $f_{\text {pi }}$ cata. Dill. eltb.79. t. 67. f. 78.

loc. Virginia, Noveboracum, Sibiria. Pereqnis, cicur, phara. ACTAEAE Radix, Eiffentia. CVAl. tetra foctida, fubvenenata.

Cauta, inuftata, beroica. vis: pellens, fudorifera, anodyna, repellens. vers: Afthenia virginica.

CONP.

$\mathrm{CHE}$.

372. ACTAEAE hujus defettu fasicatas noftras fubritui poteft. 


\section{CHELID ONIVM.}

373 CHELIDONIVM majus pedunculis umbellatis, Fl. Juec. 465. Sp. pl. 723: Fil. dan. to 542.

Chelidoniun majus vulgare. Baub. pin.144.

zoc. Europ $x$ ruderata. Peremis, vulgaris. PHARM. CHELIDONII MAJORIS Radix, Herba, $\nabla$. QVAL. fuccus luteus, amarus, acris, deurens.

ris: diuretica, diaphoretica.

Rarior, preftans.

vsvs: ICterus, Cachexia, Hydróps, Tabes, Oedema, Herpes, Pterygium.

COMP.

\section{PAPAVER.}

374- PAPAVER Rboas capfulis glabris globolis, caule pilolo multifioro, foliis pinnatifidis incifis. Ii. fuec. 468. Sp. fl. 726.

Papaver erraticum majus. Baub. pin. rq̨i.

Ioc: Europa arva.

Annua, vulgaris.

rHARM. RHOEADIS Herba, Flores, Conferva, Syrupus $z_{j}, \mathrm{R} j^{i j}, \nabla$.

QV.L. amariufcula.

vis: fubnarcotica, anodyna.

Trita, praftans.

vsvs: Pleuritis, Catarthus.

EONP.

375. PAPAVER fommiferum capfulis calycibusque glai bris, foliis amplexicautibus incifis. $S p \cdot p l .726$. Amen. acad. 8. p. $29 \mathrm{r}$.

a. Papaver hortenfe, femine nigro. Baub. pin. 160. B. Papaver hortenfe, femine albo. Bath. pin. 170.

loc: Europx auftralioris agri. Amma, cirur: pHARM. PAPAVERIS NIGRI Semina, Capitula, Syrupus (Diacodium) 3 j.

ALBI Herba, Syrupus zvj, (Q), Semina (O). 
OPIVM crudum Gr, $\ddot{j}$. correctum, Succus infpilfatus, Extractum Gr. V, 'R' cum t, R fune + .

eval. Opii lactelcens gummofo-refinola, amara, acris, tetra foctida, indammabilis. Trita, beroica. vis: narcotica, phantaftica, dementans, exhilarans, diaphoretica, anodyrna, hypnotica, antifpalinodica, antemetica, obftuens, fultens, paregorica, paralytica.

vsvs: Agrypnia, Morbi dolorifici, fpantici, evacuatorii, contagiofi, Vomitus, Phrenitis. [Syphilis.] conp. Andromach. Dialcord. Crvietan. Philon. Pil. Cynogloil. Laud. liquid. Gtt. X.

\section{A MBOGIA.}

376. CAMBOGIA Gutta. Fl. zeyl. 195. Sp. pl. 728. Coddam - pulli. Rbeed. mal. p.4I. t. 24. Loc. Malabaria, Zeylona. Arbor, peregrina. pharns. GVTTAE Gummi (Succus) Gr. X. fulphuratum Gr. xvj.

Qval. refinofo-gummofa, lutea, opaca, nitida, infipida, inodora.

vis: draftica, lyydragoga, fubemetica.

vsis: Quartana, Hydrops, Cachexia, IClerus, Afthma, 'Tania.

comp. Extr. catholicum.

376. [ Gummi Guthe verum zeylonenfe e Cambngia Gutta non provenit, fod ab alia Arbore polygama, fiuctu cerafiformi, eduli, Kenig. Retz. fufc. 4. p. o. G. Gutte americaumu vid. n. 498. Tale ciummi, indico virihus limile, e Clufin rofere fructus fucce firlfo relinofo flavo parari polfe in Inf. Bahamenfibus (ubi ideo Gombogia a nommillis appellatur,) narratum eft cel. D. Schopplio Ned. aul. Baruth.] 


\section{NYMPHAEA.}

377. NYMPHAEA alba foliis cordatis integerrimis, calyce quadrifido. Fl. fucc. 470. Sp. pl.729. Fl. dan. t. 602 .

Nymplirea alba major. Baub. pin. 193.

Loc. Europx flagna finviique. Perennis, vulgaris. PHarm. NYMPHAEAE ALBAE Radix, Flores, Conferva, Symupus, $\nabla$, (O).

Qv. Fl. fragrans. Rad acris, faponacea. Exolcta, dubia. vis: I\%. refrigerans, anodyna, hypnotica, Rad. veficatoria.

viss: Diarrhar, Gonorrhcea, Febres.

COMP.

\section{$\mathrm{BIX \Lambda .}$}

378. BIXA Orlema. Hort. cliff. 21r. Sp.pl.730. [Soinnerat. voy. p. 29. tab. 13.]

Vrucu. Marcg. braf. 6r. Sloan. jam. 2. p.52.t.181. f. I. Loc. Brafilia, Mexico.

JHARM. ORLEANA Terra (Fecule).

QVAL. fubamara, fubaromatica, rubra. Exoleta. vis: tinctoria, refrigerans, corroborans, ftomachica. vsvs: Hxmorrhagia, Prolhuvia.

comp. Chocolata, Bezetta.

\section{TILIA.}

379. TILIA europera floribus nectario deftitutis. Fl. freec. 47x, Sp. pl. 733. Fl. dan. t. 553.

Tilia femina, folio majore. Baub. pin. 426.

Loc. Europie noftre prata. Arbor, vulgaris. pharm. TILIAE Carbo, Cortex interior, Flores, Conferra, $\nabla, \Omega$.

cyar. El. fragrans.

vis: Fl. anodyna, antifpafmodica.

Rarior. 
POLYANDRIA. MONOGYNIA. I $\sigma_{3}$

vsvs: Fl. Vertigo, Epilepfia.

comp. Aqua confortans perlata, Spir. Embryon. Aqu. hirundinum.

\section{THEA.}

330. THEA bohea floribus hexapctalis. Hort. cliff: 204. Amoen.acad. 7. p. 237. t. 4. S\%.pt.734. Lett. font. Monogr. Lond. 177. Thunb. jat. 225.

Thee. Kempf. anwen. 606. t.606. Breyn. cent.rn. Bont. jav. 83. Barth. act. 4. p. I.

loc. Chind, Japonia. Arbufula, peregrina. PHARM. THEAE Folia.

Qral. lubinodora, ficca, herbaceo-flyptica.

Culinaris, untatifina. vis: exficcans, corroborans, diuretica. vsvs: Somnolentia, Polytarcia, Convulfio, Calculus; nocet ventriculo, debilibus.

rOMP:

\section{CARYOPHYLLVS.}

381. CARYOP:TYLLVS aromaticus. [foliis fubfefilibus acutis. Berg. mat. med. p. 475.] Hort. Eliff. 207. Sp.pl.735. [Sonmerat. voy.p.195́. t.110.]

Caryophyllus aromaticus, fructo clavato monopy. reno. Pluk. piojt. 155.t. I.

Ioc. Mlolucex, prexfertim Ternata. Aibst, peresrina. PHARM. CARYOHHYLI AROMATICI Fruclus, R, 0 , Balfonitis.

ANTHOPIIILLI Fruchus.

CVAL. aromatica, calidifima.

Culinaris, eximia. Anth. frperfula. vis: calcfaciens, tonica, ftomachica, tidargega, emmenagoga.

vsvs: Odontalgia, Naufea, Paralyfis, Vertigo. 
comp. Penedicta, Orvietan. El. diafatyr. Bals. apoplect. Empl. ftomachal. Spir. maftichin.

\section{CIST VS.}

382. CISTVS creticus arborefeens exflipulatus, foliis fpatulato-ovatis petiolatis enerviis icabris, caly. cinis lanceolatis. Sp. pl. 738.

Ciftus ladanifera cretica, flore purpureo. Tournef. cor. I9. it. I. p. S9. *

ı.oc. Infulx maris Aegxi, Creta. Arbufcula, fera. PHARM. LADANI GLimmi.

Qyal. Refina amara, grifea, fumo grato. Rarior. vis: paregorica, flomaclica.

vsvs: Dylenteria, Catarihus.

comp. Bals. apoplecticus.

\section{$D I G I N I A$. PAEONIA.}

383. PAEONIA officinalis foliolis oblongis. Hort. cliff. 212. ups. 149. Sp.pl. 747 .

a. Pæonia folio nigricante fplendido, qux mas. Baub. pin. 323 .

B. Pronia communis vel femina. Bålb. pin. 323. roc. Alpes Helveticx. Perennis, cicur. pharm. $\propto$. PAEONIAE Radix, Fecule, Conferva. Syrupus, $\mathrm{R}, \nabla$, Semina. $\beta$. PAEONIAE Flores, Conferva, Syrupus. QVAL. tetra, fœtida. Vjitata, beroica. vis: fubaditringens, narcotica, antilpalinodica, anodyna, hypnotica, pellens, emmenagoga. vsvs: Convulfiones, Ecclampfia, Epilepfia. conr. 专 gutte; 专 epilept. Marchion. Sp. embryon. et Aqu. hirund. 


\section{$T R I G I N I A$. DELPHINIVM.}

384. DELPHINIVM Confolida nectariis monophyllis caule fubdivifo. Fl. Juec. 476. Sp. pl.748. Fl. dan. t. 683 .

Confolida regalis arvenfis. Baub. pin. 143 .

loc. Emopx agri. Anmu, vulgaris. PHARM. COMSOLIDAE REGALIS Flores.

CVAL. carulea. Exoleta, Juperflua.

ris : dubia.

risis:

comp. Syrupus violarum fuccedaneus.

385. DELPHNIVM Staphifagria nectariis tetraphyllis petalo brevioribus, folis palmatis: lobis obtulis. Hort. cliff. 213. ups. 150. Sp. pl. $75^{\circ}$.

Staphifigria. Brib). pin. 324 .

Loc. Iltria, Dalmatia, Cálabria, Apulia.

PHARM. STAPHISAGRIAE Semina.

Biennis, manjueta.

ovil. amara, nauleofa. Trita, preftens, externa. vis: draftica, exanthematica, anthelminthica, phthiriaca.

vsvs: Pilces, Pediculi, Acari fubcutanei, Odontalgia. coMP.

\section{ACONITVM.}

386. ACONITVM Napellns foliorum laciniis linearibus. fuperne lationibus: linea exaratis. Hort. clijj. 214. Fi. fuec. 477. Sp. pl. 751. Act. Jtockb. 1739. p. 41. t.2. ofacy. auflr. 4. t. $38 \mathrm{I}$.

Aconitum cxruleum f. Napellus 1. Baub. pin.183. roc. Europa humidiulcula. Perenmis, cicur. PHARM. NAPELLI IHaba, Extractum gr. $\beta$ al. i. L 3

QVir.o 
Qval. fubfyptica, acris, naufcofa, toxica. Heroica. vis: corrodens, veficans, excorians, vomitoria, draftica, convulfiva, vertiginola, fternutatoria, peliens, fudonilera.

vsva: Fchres intermittentes, Ifchias, Arthritis, Rhcimatifmus, Scirhus, Anchylofis. COMP.

387. ACONITVM Anthora foribus pentagynis, fo. liorum laciniis linearibus. Sp. pl. 751. Facq. aufti. 4. $t .382$.

Aconitum Calutiferum f. Anthora, Baub. pin. 184. zoc. Alpes Europxx.

PHAR M. ANTHORAE Radix $\mathfrak{z}$ j.

NHAt. amara, acris, toxica. Exoleta, dubia. vis: juigans, exanthematica, anthelminthica, Antti-Thora.

vsvs: Febres exanthematica.

comp. Orvietanum.

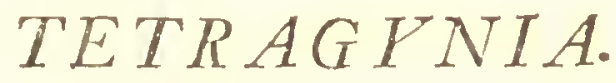 CIMICIFVGA.}

388. CIMICIFVGA fotida. Amon acad. 7. p. 435.t. 6.f.2. 8. t. 193. t. 4. Gmel. fib. 4. p. 181. t. 70. Loc. Sibiria.

PHARM. CMILIFUGAE Herba.

eral. tetcrima, acris. Imufitata, preftans. 'ris: anodyna, pellens, repellens, emetica.

rsvs: Morbi nervorum, Hydrops, Arthritis, Struma. COMP.

38. Cleome gigantea odore foctidn Cimicifugam fuperat, fapore ila acri, 4 vix hue vumu mafticari polfit; unde ad vires ejus heroicas concludere licet. 


\section{$P E N T A G Y N I A$. A QVILEGIA.}

3S9. AQVTIEGIA vulgaris nectariis incurvis. Hort. upl. I52. Fl. Juec. 478. Sp.pl.752. Fl. dan. t.695. Aquilegia fylveftris. Baul. pin. I44.

roc. Europa.

Perennis, cicur. PHARM. AQVILEGIAE Herba, Flores; $\nabla$, Semi. na 3 ?.

QTAL. herbacea, toxica. Rarior, Hb. Supergutu. ris : pellens, cranthematica.

rsvs: Exanthemata, Icterus! Dyftocia, Scabies.

cons. Syrup. viol. fuccedaneus.

\section{NTGELLA.}

390. NiGELLA fativa piftillis quinis, capfulis muricatis fubrotundis, foliis fubpilofis. Hort. upe. $154^{\circ}$ Sp. pl. 753 .

Nigella itore minore fimplici candido. Bauh. pin. 145. Loc. Creta, Aegyptus.

pharm. Nigeline Semina, (D).

QYAL acris, fragrans, toxica. Annua, manfueta. vis: jellens, errina, fialagoga, nernutatoria, emmenagoga, laclifera, anthelminthica.

vsve: Coryza, Catarrhus.

COMP.

\section{POLYGPIIA. WINTERA.}

391. WINTERA aromatica pedunculis aggregatis terminalibus, pintillis quatuor. Suppl. 269. Sy/t. ver. I4. 1.507. Soland. med. oly. and. inquir. 5. p. 4 \%. t. I. 
Drimys Winteri. Forft. gen. 84. Nov. act. upfal. 3. p. 181 .

Cortex vinteranus. Cluf. exot. 75.

soc. Terra Magellanica.

PHARM. WINTERANVS Cortex.

Arbor, fera.

Cval. acerrima, pungens, aromatica, fragrans.

Rarior, cximizo.

vis : calefaciens.

vsvs: Scorbutus!

COMP.

\section{ILL I C I V M.}

392. ILLICIVM anifatum fluribus flavefcentibus. Sy/t. nat.2.p. 422. 'Tímb.jap. 235.

Skimmi. Krempf. amen.880, 1.881.

Loc. Tataria, China, Philippins.

Fera.

PHARM. ANISI STELLATI Fruckus, Effentia.

QVAL. aromatica, dulceferns, fapore anifi.

Trita, preftans.

vis: Aomachica, carminativa, diuretica, pectoralis. vsvs: Calculus, Tuflis.

comp.

\section{ANEMONE.}

393. ANEMONE Hepatica foliis trilobis integerrimis. Fl. flec. 480. Sp.pl. 758. Fl. dan.t.610. Trifolium hepaticum, flore fimplici caruleo. Baub. pini. 339.

Loc. Europx fylvx opaca. Perennis, vulgaris. pharm. HEPATICAE NOBILIS Herba, Flores. er. inodora, infipida. Exoleta, preftans, Fil. Juperfun.

Vis:

391. [MINTERANVS Cortex craflus, externe levis, cinerens, jurus fibrofus, brunneus, acerrinus, pungens, ferwdior liuguam magis dintiusque mordens, minus vero amarits et aromaticus Canella albe Cortice, odore ad Cafcarilliz Cort. fere accedit; benevole ccmmunicatus a celeberr. Forlleris.] 
VIS: adfringens, tonica.

vors: Hypochondriafis, Hemia.

CUMP.

394. ANEMGNE Pulfatilla pedunculo involucrato, petalis rectis, foliis bipinnatis. Fl. Juec.481. St. pl. 759. Fl. dan. t. 153 .

Pullatilla fulio craffiore, majore flore. Baub. pin. 777 . I.oc. Europx colles aprici. Perennis, vulgaris. PharM. PVLSATILLAE Herba, $\nabla$.

QFAL acerrima toxica.

Vis: corrodens, -rubefaciens.

Exoletr, fuperfun.

rois: Febres.

contr.

395. $\triangle \mathrm{NEMONE}$ nemorofa feminibus acutis, foliolis incilis, caule unilloro. Fl. Juec. 485. Sp.pl. 762 . Fl. dan. $t .5 \div 9$.

Anemone nemorofa, flore majore. Baub. pin. r76. Loc. Europe nemora prataque verna. Perennis, freq. PHARM. RANVMCVLI ALBL Flores, $\nabla$.

QVAl. acris, toxica.

Rarior.

vis: corrodens, colmetica.

rsvs: Cephalalgia.

COMP.

\section{CLEMATIS.}

396. CIEMATIS recta foliis pinnatis: foliolis ovatolanceolatis integerimis, caule crecto, Horibus pentancialis tetrapetalisgue. Hort. cliff. 225. Sp. pl. 767. facy. aufir. 3. 1.49. t. $29 \mathrm{r}$.

Iilanmula. Cam. epit. 608. recta. Baub. pin. 300.

loc. Germanix, Galiix colles. Pereruis, cicur. I. 5

I'HARA.

394. [Frequentius adhibetur Anemone pratenfis r. I'nllalilla nigricans, in morbis oculoram, preeunte Perill. "Sibil 
pharm. FlaMMVLAE JOVIS Herba, Flores. QVÁL. acris.

vis: diurctica, fudorifera, laxans, veficans. vsvs: Vlcera cancrofa e Syphilitide.

cosp.

\section{RANVNCVI, VS.}

397. RANVNCVLVS Ficniria foliis cordatis angulatis petiolatis, caule unilloro. Fl. fuec. 496. Sp. pl. 774. Iil. dan. t. 499.

Chelidonia rotundifolia minor. Baub. pin. 309. Loc. Europe umbrofa, culta. Perennis, vulyaris. pharm. CHELIDONII MNORIS Radix, Herba. cval. Inbinfipida, oleracea. Exoleta, dubin, fuperflua. vis: colinctica, [diuretica.]

vsvs: Varix ani? [Scorbutus.]

COMP.

\section{HELLEBORVS.}

398. HELLEBORVS niger fcapo fubbifloro fubnudo, foliis pedatis. Hort. 2ups. 157. Sp.pl. 783. [Scbmidel. ic. p. z6. t. 6.]

Helleborus niger, flore rofeo. Baub. pin. Is6.

Loc. Alpes Apennine, Auftria, Hetrurix.

Perennis, manifueta.

PHARM. HELLEBORI NIGRI Radix Bj. Fibrx, Extractum $3 \beta$.

QVAL. acris, amaricans, fubnaufeofa, fortida. Trita, eximia.

ris:

398. [Hellebaro nigro frepe fubfituntur Helleborts wiridis, cujus rad. amarior, acrior magisque naufeofa; Adoris vernulis (Helleb. Hippocratis falfo dicta), fifteca fyicata, Frol-

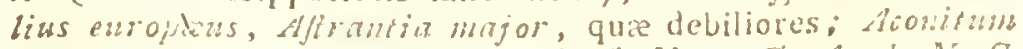
N'ap.llus, qua venenatiffima, Conf. Nov. ali. Acad, N.C. vo6. G. p. Ifq. $\int q q . J$ 
POLYANDRIA. POLYGYNIA. ITI

VIs: purgans! pellens, emmenagoga.

virs: Mielancholia, Hypochondriafis, Mania, Chlorofis, Achor.

comp. Faxtr. cathol. Extr. panchymagog. Pil. Starkej.

394. HELLEBORVS fotidus caule multifloro foliofo foliis pedatis. Hort. cliff. 227. Sp. pl. 227.

Helleborus niger fætidus. Baub. pin. 185.

LOc. Germania, Gallia. Peremis, cicur. PHARM. HELLEBORI FOETIDI Herba.

QYA. acris, tetra.

vis: purgans, emetica, anthelminthica.

vsus: Vermes.

CONIP. 


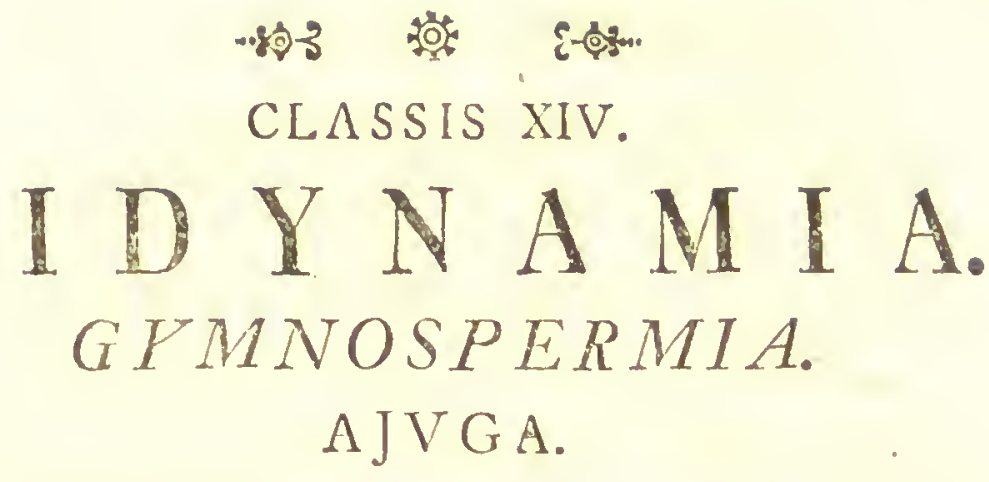

400. AJVGA fyramidalis tetragonn-pyramidalis villofa, foliis radicalibus maximis. Fl. Juec. 512. Sp. pl. 795 . Fl. dar. t. 185.

Confolida media genevenfis. Baub. bift. 3. p. 432.

Lor. Europa prata montana, vix. Biennis, vulgaris. PHARM. CONSOLIDAE MEDIAE Herba.

QYAL. amara.

Exoleta, dubia.

vis: fubadflingens, vulneraria.

vsve: Contufura.

COMP.

\section{TEVCRIVM.}

401. TEVCRIVM Clamepitys foliis trifidis linearibus integermimis, floribus fellilibus lateralibus folitariis, caule diffufo. Hort. ups. 160. Sp. pl. 787 . Fl. dan. t. 733 .

Chamapitys lutea vulgaris $\int$. folio trifido. Baub. pin. 249.

coc. Italix, Anglix, Aufrix agri. Anmu, cicur. PHARA. CHAMAEPITYOS Herba.

Qyil. refinofo-aromatica, amara. Trita, preftans. ris: tonica, ftomachica, refolvens, pellens, emmenagoga.

vsws: Rheumatifmus, Arthritis, Podagra, Hæmaturia? comp. Andromach.

402. TE-

400 [Confolide media herbam pharmacopolis extra Sueciam - imega reprans rulminifrat.]

401. Ad AJVGAE genus potius referenda eft, monente b. Hallra. Sinreb. unilab. s.t.] 


\section{DIDYNAMIA. GYMNOSPERMIA. I73}

402. TEVCRIVMI creticum foliis lanceolato-linearbus integerrimis, Horibus racemofis ternis. Sp. $\mu .788$. Rolmarinum ftoechados facie. Alp. exot. 103. f.roz. Loc. Cireta.

PHAR.I. POLII CRETICI Herba. Suffrutex, manfiuetus. CYAL. amara. Exoleta, debilis, fuperflua.

vis: tonica. vsrs: Calculus, ICterus.

conp. Andromach. Mithridat.

403. TEVCRIVM Marnm foliis integurrimis oratis acutis petiolatis fubtus tomentofis, floribus racemofis fecundis. Sp. pl. 789. Amon. acal. 8. p. 221. Marum cortufi. Baul. bift. 3. p. 242.

Ioc. Regnum Valentinum. Suffrutex, manfuetus. PHARM. MARI TERI Herba, Folia f. f. Effentia. UVAl. fpirans, acutiflima, amara. Rarior, cara, beroica. Vis: refolvens, nervina! pellens, diuretica, emme. nagoga, errhina, cordialis, tonica.

vsvs: Cachexia, Hydrops, Afthma! Oblivio, Soporofi! Anofmia, Hyfteria.

CONP.

404. TEVCRIVM Scordium foliis oblongis fefilibus dentato-ferratis, Horibus geminis axillaribus pe. dunculatis, caule diffulo. Fl. Juec. 513. Sp.pl. 790. Fi. dan. t. 593 .

Scordium. Baulb. pin. 247.

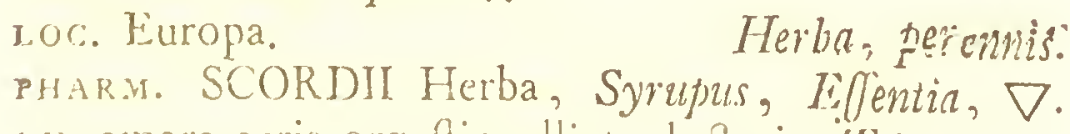
⿺辶. amara, acris, orgaftica alliata, lactaria. Trita, exim. vis: balfamica, tonica, antiputredinofa! alcxiteria! antifebrilis, refolvens, pellens, diaphoretica, anthclminthica.

VSVS:

403. [Polism creticnn veram eft $T$. Touthrion Schrak. anil. 46.] 


\section{I74 DIDYNAMIA. GYMNOSPERMIA:}

vsvi: Gangrana! Febres, Peftis, Vlcus.

comp. Ther. Alexipharm. Andromach. Orvietan. Mithridat. Diafcordium. EEfent. alexipharm. Stablii.

405. TEVCRIVM Clamedrys foliis cuneiformi-ovatis incifis crenatis petiolatis, floribus ternis, caulibus procumbentibus fubpilofis. Hort. cliff. 302. Sp. pl. 790.

Chanxdrys major (minorque) repens. Baub.pin. 248. roc. Italia, Gallia, Anglia, Germania, Helvetia.

PHARM. CHAMAEDRYOS Herba.

Perennis, manflieta.

QVAL. fpirans, amara.

Trita, preftarss. vis: balfamica, tonica, fomachica, pellens, fudorifera, cmmenagoga.

vsvs: Arthritis,' Podagra, Tertiana! Scrophulx,

Catarrhus, Chlorofis.

comp. Andromach.

\section{SATVREJA.}

406. SATVREJA bortenfis pedunculis bifloris. Virid. cliff. 87. Hort. upj: i6r. Sp. pl. 295.

Satureja hortenfis. Baub. pin. 218.

LOC. Monfpelium.

Annus, sicur.

pHARM. SATVREJAE Herba, $\nabla, \circ 0$, Semina.

QVAL. fpirans, aromatica. Rarior, preftans.

vis : refolvens, excitans, pellens, ftomachica, emmenagoga, aphrodifiaca.

vsvs: Anorexia, Atecnia.

COMP.

407. SATVREJA capitata floribus fpicatis, foliis ca-; rinatis punctatis ciliatis. Sp. pl. 795 .

Thymus capitatus, qui diofcoridis. Bauh. pin. 219. 
DIDYNAMIA. GTMNOSPERMIA. I75

roc..Grecia, Creta, Brtica, Hilpalis, Gades.

PHARY. THYMI CRETICL Herba.

Suffrutex, ferus.

Qv. fpirans, Thymo 427. 428. fortior. Exoleta, preft. vis: Thymo vulgari 428. fortior.

isis:

Cusir.

\section{H Y S S O P VS.}

408. HYSSOPVS officinalis fpicis fecundis, foliis lanceolatis. Sp. pl. 796. Facq. auftr. 3.t. 254.

Hyliopusonticinarum cerulea 1. Ppicata. Buth.pin.2r\%. Loc. Auttia fubalpina, Rullia auftralis Suffrutex, cicar PHARr. HYYSSOPI Herba, Conjerva, Syrupus, Ef. Sentia, $\nabla: \circ$ o , Semina.

Qr A L. amaricans, nidorofa halituofa. Trita, preftans. vis: ballamica, tonica, refolvens, expectorans, ftomachica.

vsvs: Tuins, Angina, Afthma humorale, Poda. gra, Calculus, Ophthalmia, Sugillatio. coirp: Syr. HylTopi.

\section{NEPETA.}

409. NEPETA Cataria floribus fpicatis, verticillis fubpedicellatis, foliis petiolatis cordatis dentatoferratis. Fi. Juec. 514. Sp. pl. 796. Fl. dan. t.580. Mentha cataria vulgaris et major. Bauh. pin. 228. Loc. Europe pagi, ruderata. Peremis, rulgaris. PHARYA TESETAE Herba.

Qvir. hircola felina, amara. Exoleta, preftans. VIs: refoliens, pellens, cephalica, cmmenagoga,

auticumisthica.

vers: Hyfteria, Chlorolis!

comp. Injelfus. 


\section{A V A N D VLA.}

4ro. LAVANDVLA Spica foliis lanceolatis integris, Ipicis nuciis. Hort. cliff. 303. upf. 162. Sp. pl.800. Lavandula latifolia et angultifolia. Baub. pin. 216. loc. Italia, Hilpania, Gallia narbonenfis.

Suffrutex, fubcicur.

pharm. LAVANLVLLE Flores, Conjerva, $\nabla, \widetilde{V}, \circ$ o. SUICAE O०.

Qval. amara, fpirans.

Trita, eximia.

vis: nervina, relolvens, cephalica, uterina.

vsvs: Aphonia, Balbuties, Dyltocia.

comp. Balf.lavandulx, Balf. apoplect. Spir. embryon.

4Ir. LAVANDVLA Stoechas foliis lanceolato-linearibus integerrimis, fpicis comofis. Hort. cliff. 303. upp. 162. Sp.pl.800.

Stoechas purpurea. Baub. pin. 216 .

Loc. Hilpania, Lufitania, G. Narbonenfis.

Suffrutex, manfuetus.

PHARM. STOECHADIS ARABICAE Flores (Spica rectius).

QVAL. firans, prxcedenti graveolentior. Exxoleta. vis: cephalica, nervina.

vsvs: Paralyfis, Vertigo, Hypochondriafis.

comp. Mithridatium.

\section{MENTHA.}

412. MENTHA Auricularia fpicis oblongis, foliis oblongis ferratis pilofis feflilibus, ftaminibus corolla longioribus. Mantif. fl. 8r. Fl. zeyl. 48.* Majorana fotida. Rumph. amb. 6. p.41. t. 6 . coc. Indix orientalis aquofa. Fera. PHARM. MENTHAE AVRICVLARIAE Herba. CVAL. amara, fpirans.

Ignota. vis : refolvens. 


\section{DIDYNAMIA. GYMNOSPERMIA. I7?}

vsve: Copholis, Tumores.

COMP.

413. MENTHA fylveftris fpicis oblongis, foliis oblongis tomentofis ferratis leililibus, faminibus corolla longioribus, Sp. pl. 804. Fl.dan. t. 484. Mentha fylveftris, folio longiore. Baitb. pin. 227. Loc. Germania, Anglia, Gallia. Perennis, cicur. PHARM. MENTHAE SYLVESTRIS Herba. QYAl. amaricans, fpirans. Rarior, externa. vis: fequente debilior, magisque adhringens. vsvs: Calculus, Hyfteria, Colica, Vomitus. comp. Epithemata.

4I4. MENTHA crifpa floribus capitatis, foliis cordatis dentatis undulatis feflilibus, faminibus corollam rquantibus. Hort. cliff. 306. upp. 168. Sp. pl.805. Mentha crilpa danica f. germanica fpeciofa. Morif. bift.3. p. 367 . . . iт. t. 6. f. 5 . Loc. Europa.

Perennis, cicur. PHARM. MENTHAE CRISPAE Herba, Conferva, Syrupus, $\nabla, \Omega$, oo, (). QVAr. amaricans, fpirans. Trita, eximia. vis: relolvens, pellens, camminativa, ructatrix, antaphrodifiaca, emmenagoga, lac repellens, ftomachica.

vis: Soporofi, Cephalalgia, Vomitus, Colica! Anorexia! Licnteria, Hyfleria, Dylmenorthoa, Leucorrha, Afthma, Tufis convulfiva! Bubo mammarum, Satyriafis.

comp. Spir. embryon.

415. MENTHA pipcrita foribus capitatis, foliis ovatis ferratis petiolatis, flaminibus corolla brevio. ribus. Sp. pl. S05. [Knigg. difJ.t. I.] 
I7S DIDINAMIA. GYMNOSPERMIA.

Mentha fpicis brevioribus ethabitioribus, foliis men. thr fulcx, fapore fervido piperis. Raj. angl. 3 . p. 234 . t. 10. f. 2 .

roc. Anglia.

Perennis, cicur. PHARM. MENTHAE PIPERITAE. Herla.

Qral. pracedentis, fed acrior, cum lenlu refrigerii.

vis: prxcedentis, fed fortior.

Rarior, eximia.

vsve: pracedentis, praftantior.

COMP.

416. MENTHA Puleginu Horibus verticillatis, foliis ovatis obtulis fubcrenatis, caulibus fubteretibus repentibus, faminibus corolla longioribus. Hort. cliff. 306. Sp. pl. 807 .

Pulegium latifolium. Baub. pin. 222.

coc. Germanix, Anglix, Gallix fublumida.

pharm. PVLEGII Herba.

Perennis, manfueta.

QVal. M. crifpe, fed acrior. Vfitata, proftans. vis: $M$. crifpe, fed fortior, minusque adftringens, emmenagoga.

vsvis: A thma, Tuflis convulfiva, Raucedo!

COMP.

\section{GLECOMA.}

417. GLECOMA bederacea foliis reniformibus crenatis. Fl. fuec.518. Sp. pl. S07. Fl. dan. t. 789.

Hedera terreftris vulgaris. Baub. pin. 306.

Loc. Europx fepes, dumeta. Peremis, vulgaris. PHARm. HEDERAE TERRESTRIS Herba, Conferva, Syrupus, $\nabla$. QVAL. amaricans, fubacris. Trita, debilis. vis: Tonica, vulneraria. 
DIDYNAMIA. GYMNOSPERMIA. I79

rsvs: Vlcus internum, Colica hypochondriaca, Tullis, Cephalalgia.

coms.

\section{A M IVM.}

418. LAMIVM album foliis cordatis acuminatis ler. ratis' petiolatis, verticillis vigintifloris. Fl. Juec. 520. Sp. pl. Sog. Fl. dm. t. 594 .

Lamium album non fætens, folio oblongo. Baub. pin. 231 .

loc. Europe, ruderata culta. Anmua, vulgaris. PHARM. LAMII ALBI Herba. QVAl. fubfatua, oleracea, tetra. Lixoleta, dubia. vis :

vsvs: Leucorrhoea.

COMP.

\section{BETONICA.}

4I1). BETONICA offcinalis fpica interrupta, corollarum labii lacinia intermedia emarginata. Fl. fuec. 515. Hort. ups. 164. Sp. pl. Sio. Fl. dan. t.726. Betonica purpurea. Baub. pin. 235.

Loc. Europa aultralior.

Perennis, cicur. PHARM. BETONICAE Herba, Flores, Conferva,

Syrupus, $\nabla$.

RVAL. ficca, nidorofa halituofa. Rad. naufeofa. vis: nervina, tonica, errhina. Rad. emetica.

vsvs: Cephalalgia, Hyfteria. Trita, debilis.

cOMP.

\section{ST $\triangle$ C H YS.}

420. STACHYS fylvatica verticillis fexfloris, foliis cordatis petiolatis. Hort. cliff. 300. Ir! Juec. 526 . Sp. pll. 8II.

\section{$\mathrm{M} 2$}

420. [Stachys refta, Siderifis officin. in Gormania paftim in numen rum medicamentorum recipitur. Vfus: in Rhachitide externe.] 


\section{I80 DIDYN AMIA. GYMNOSPERMIA.}

Lamium maximum fylvaticum foctidun. Baub.pin. Loc. Europx nemora. Anmua, vulgaris. PHARM. GALEOPSIDIS Herba.

CVAL. tetra fotida. Exoleta.

vis: anodyna, vulneraria.

vsvs: Pleuritis, Nephritica.

COMP.

\section{MARR VBIVM.}

42r. MARRVBIVM vulgare dentibus calycinis fetaceis uncinatis. Fl. fuec.531. Sp.pl. 816.

Marrubium album vulgare. Baub. pin. 230.

r.oc. Europa ruderata, argillofa. Perennis, vulgaris. I'HARM. MARR VBII ALBI Herba.

Qv. graveolens, amariufcula, ingrata. Exoleta, preftans. vis: pellens, emmenagoga, thoracica, anthelminthica.

vsvs: Afthma humorale, Ptyalifmus mercurialis, Obftipatio vifcers, Dysmenorrhœa, Hyfteria. comp. Syr. Prafii, Pil. Agarici, Andromach.

\section{LEONVRVS.}

422. LEONVRVS Cardiaca foliis caulinis lanceolatis trilobis. Fl. fuec. 532. Sp. pl. 81\%. Fl. dan. t. 627. Marrubium Cardiaca dictum. Baub. pin. 230. ıос. Europæ ruderata, pagi. Biennis, frequens. pharm. CARDIACAE Herba, $\nabla$. QVAL. ficca.

Exoleta, infida.

VIS : roborans.

vsvs: Hyfteria! Hypochondriafis?

COMP.

\section{O RIGA N V M.}

423. ORIGANVM Dictamm foliis inferioribus to. mentofis, Spicis nutantibus. Hort. cliff.jr4. Sp. pl. $8 \dot{3}$. 
DIDYNAMIA. GYMNOSPER,MIA. I8I

Dickamnus creticus. Baqb. pin. 222.

Loc. Mlons ida Cretx. Suffrutex, ferus. PHAR.M. DICTAMNI CRETICI Folia.

Cral. Jpirans, calida. Trita, eximia. vis: refolvens, pellens, nervina, uterina, emmenagoga.

rsvs: Dyftocia.

comp. Andromach. Mithridat. Orvietan. Diafcord.

424. ORIGANVM creticum fpicis aggregatis longis prifmaticis rectis, bracteis nembranaceis calyce duplo longioribus. Sp pl.823.

Origanum creticum. Baul. pin. 223.

ioc. Creta.

Herba, peregrina. PHARM. ORIGANI CRETICI Herba (rectius Spi(a), .00

Qval. lpirans, calida.

Trita, eximia.

vis: ftimulans.

vsvs: Odontalgia.

comp. Andromach.

425. ORIGANVM vulgare fpicis fubrotundis paniculatis conglomeratis, bracteis calyce longioribus ovatis, Fil. fuec.534. Sp. pl. 824. Fl. dan. t. 638 . Origanum fylvefre. Baub. pin. 223.

1.oc. Europx montes.

PHAR.M. ORIGANI VVLGARIS Herba.

QVar. amaricans, fubacris, tinctoria, fpirans. Rnvior. vis: corroborans, abftergens, refolvens, excitans. vsis: Tuflis, Althma, Phthilis, Chlorofis, Oedema! conp. Syr. artcmifix compofitus; Balnea uterina. libere fiscedanea.

426. ORIGANVM Majorana foliis ovatio obłufis, fpicis fubrotundis compadis pubefcentibus. Hort. cliff. 304. upf. 16r. sp pl. 826. 
$\$ 82$ DIDYNAMIA. GYMNOSPERMIA.

Majoiana vulgaris. Baub. pin. 224.

LCC.

Annua, fubmanfueta.

phakm. M \JORANAE Herba, Con/erva, $\nabla, \circ$.

QVAr. amuricans, fpirans. Culinaris, proftans.

vis: tonica, refolvens, nervina, cephalica, ptar-

mica, ftomachica.

vsvs: Coryza, Anofmia.

cомг. Balf. majorane, Balf. apoplecticus, f fter. nutatorius,

\section{THYMVS.}

427. THYMVS Serpyllum floribus capitatis, icaulibus repentibus, foliis planis obtufis bafi ciliatis. Fl. Juec. 535. Sp. pl 825 .

Serpyllum vulgare minus. Baub. pin. 220.

Loc. Europx noftrx colles nudi. Suffrutex, vulgaris. SHARM. SERPYLLI Herba, $\nabla, \circ$ (florum), Conferva.

Cual. ftyptica, fpirans.

Exoleta:

vis: refolvens, excitans, tonica.

rsvis: Cephalalgia crapulx.

cons. Balnea uterina.

428. THYMVS vulgaris erectus, foliis revolutis ovatis, floribus verticillato-fpicatis. Hort. cliff. 302 . upf. 160. Sp. pl. 825 .

Thymus vulgaris, folio tenuiore. Bauh. pin.21g

Loc. Gallix narbonenfis exarida. Suffrutex, cicur. rharm. 'THYMI VVLGARIS Herba, $\nabla$.

QVAL. aromatica, fpirans. Vfitata, preftars. V1s: refulvens, excitans, pellens, nervina, corro.

borans, tonica, flomachica, eminenagoga.

vsvs: Senectus, Leucophlegmatia, Coryza, Epiphora. 


\section{MILLIS SA.}

429. MELISSA officinalis racemis axillaribus verticillatis, pediccilis fimplicibus. Sp. pl.827.

Melinia hoitenfis. Baub. fin. 229.

loc. Italia Allobrogica montes.

Perennis, cicers. IHARM. MLLISSAE CI'TRATAE Herba, Syrupus, lilientia, $\nabla, \widetilde{V}, \stackrel{0}{\mathrm{~V}}$.

crai. Pirans: odore cort. citri. Trita, eximia. vis: reiolvens, excitans, pellens, cordislis, tonica, cmmenagoga, diuretica.

rsve: Melancholia, Hypochondriafis, Hyferia, Palpitatio.

comp. Spir. embryon. Acet. propliylact.

430. NELISSA Calamintiba pedunculis axillaribus di. chotomis longitudine foliorum. Sp.pl.827.

Calamintha vulgaris vel officinarum germanix. Baulb. pin. 228.

loc. Italix, Hifpanix, Gallix montes.

Perennis, manfueta. rharm. CALAMINTHAE MONTANAE Herba. QVAL. odor pracedentis: debilior. Exoleta, preftans. vis: reblvens, pellens, expectorans : ftomachica. vsis: Afthma.

coms.

\section{DRACOCEPHALVM:}

431. I)RACOCEPHALVM canarienfe floribus fpicatis, foliis compolitis. Hort. : liff. 308 . Sp. pl. 829.

$$
\mathrm{N}_{4} \text { Ceiro. }
$$

430. CALAMINTHAE MONTANAE lnco in noftris officinis male CLINORODIVII valgare fubfttuitus.

43r. DRACOCFPHALVM requens 14.32. vilgo in Pharnacopoliis fervatur, led vix, umquam proferibitu, in elijus locum ego potius hanc 43!. Subfinorcon: qua fragrantifima $i$, fa Mlarum excijias, vix laa en habet. 
184 DIDYNAMIA. GYMNOSPERMIA.

Cedronella canarienfis vifcola, floribus plerumque ex eodem pedunculo ternis. Comm. bort. 2. p. $8 \mathrm{r}$. t. $4 \mathrm{r}$.

roc. Infulx Canarix.

Peremis, manfueta. pharar. MílissaE CaNARIAE Herba. QVAL. Spirans, aromatica. vis: nervina, refolvens.

vsvs: Cachexia, Cephalalgia. C()MP.

432. DRACOCEPHALVM Moldavica floribus verticillatis, bracteis lanceolatis: ferraturis capillaceis. Hort. up $\%$. $166 . S p . p l .830$.

Melifla peregrina, folio oblongo. Baub. pin.229. roc. Moldavia, Ruthenia. Ammua, cicur. pharm. MELISSAE TVRCICAE Herba, Semina. Qrat. Cpirans. Exoleta, debilis, Juperfua. vis: nervina, refolvens.

VSTS:

COMP.

\section{O C IMVM.}

43j. OCIMVM Bafilicum foliis ovatis glabris, calycibus ciliatis. Hort. cliff. 315. upf.168. Sp. pl. 833 .

Ocimum caryophyllatum majus. Baub. pin.226. Loc. 'Leylona, India. Ammu, cicur. Phard. BASILICI Herba, $\nabla$, Semina.

QVAl. fpirans. Sem. mucilaginofa. Culinaris, preftans. vis: nervina, cordialis, pellens.

vsvs: Vomitus, Deftillationes.

comp. Ving. martiatum.

\section{PR VNELLA.}

434. PRVNELLA vulgaris foliis omnibus ovato-oblongis forratis petiolatis. Fl. Juec. 540. Sp.pl.837.

Prunel. 
DIDYNAMIA. ANGIOSPERMIA. 185

Prunella major, folio non diffecto. Baub. pin. 460. Loc. Europe prata, pafcua. Perennis, vulgaris. PHARM. HRVNELAE Herba, $\nabla$.

Q $\doteq 1$. fubodorata.

Exoleta, Superfua.

ris : fubadnringens.

rsvs: Angina.

comp. Gargailimata.

\section{ANGIOSPERMIA. EVPHRASIA.}

435. EVPHRASIA offeinalis foliis ovatis lineatis argute dentatis. Fl. Juec. 443. Sp. pl. 841.

Euphrafra officinarum. Bauth. pin. 233.

LOC. Europx pafcua fterilia. Amma, vulgatiffina. PHARM. EVPHRASIAE Herba, Conserva, $\nabla, \Omega$. CVAl. pinguis, fubamara. Exoleta, infida. vis: [fubadfringens.]

vsvs: Gphthalmia?

CUMP.

\section{PEDICVLARIS.}

436. PEDICVLARIS paluftris caule ramofo, calycibus criftatis callofo-punctatis, corollis labio obliquis. Fl. Juec. 551. Sp. pl.845. Fl. dan. t.225.

Pedicularis pratenlis rubra elatior. Raj. bift. 700.

ioc. Europax palcua paludofa. Amma, vulgaris. PHARM. PEDICVLARIS AQVATICAE Herba recens.

Qval. ingrata. Iningitata.

vis: vulneraria.

vsvs: Fiflula, Vlcus callofum:

Cusip.

$$
M 5 \text { ANTIR }
$$




\section{ANTIRRHIN VM.}

437. ANTIRRHINVM Linaria foliis lanceolato-linearibus confertis, caule crecto, fpicis teminalibus fellilibus, floribus imbricatis. Iil. Juec. 557. $S p \cdot p l .858$.

Linaria vulgaris lutea, flore majore. Baub. pin.212. roc. Europx muri, fabulofa. Perennis, oulgaris. PHARM: LINARIAE Herba, Vinguentum.

QVAL. hircofo - urinofa, amariufcula. Exoleta, eximia. vis: anodyna, pellons, repellens, eccoprotica, diuretica, purificans.

vsve: Ophthalmia, Icterus, Varix ani, Hydrops. COMP.

438. ANTIRRHINVM majus corollis ecaudatis, floribus fpicatis, calycibus rotundatis. Hort. 2ups. 175.

Sp. pl. 859 .

Antirrhinum arvenfe majus. Baub. pin. 212. Loc. Gallix, Italix agri. rHARM. ANTIRRHINI Herba.

CVAL. fatua.

vis: dubia.

vsvs: Magia.

COMP.

\section{S C R O PHVLARIA.}

459. SCROPHVLARIA nodofa foliis cordatis trinervatis, caule obtufangulo. Fl. Juec. 560. Sp.pl.862. Scrophularia nodofa fotida. Bauls. pin. 235. noc. Europe fepes. Perennis, vulgaris. PHARM. SCROHHVLARIAE FOETIDAE Radix. QYAL. tetra, fotida, amara. Rarior, preftans, vis

438. [Pro ANTIRRHINI Herba Fl. Saes. 559. et Plarmo dan.

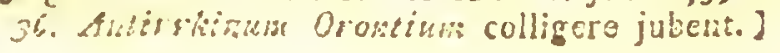




\section{DIDYNAMIA. ANGIOSPERMIA. I8?}

ris: anodyna, repellens, fudorifera, carminativa, anthelminthica!

vsis: Scropinula, I Tarix ani, Vulnus, Scabies。 Angina.

coмP. Ammletum.

440. SCR OPHVLARIA aquatica folits cordatis obtufis petiolatis decurrentibus, caule membranis angulato, racemis terminalibus. Hort. ups. 177. Sp.pl. 854. Lil. dan. t. 507 .

Scrophularia aquatica major, Bauh. pin. 235.

loc. Europa auftralior. Perennis, cicar. PHARM. SCROPHVLARIAE AQVATICAE Folia. CVAl. minus foctida. Exoleta. vis: fudorifera, carminativa.

vsis: "Tormina.

romp. Sennam corrigens.

\section{LIN NAEA.}

441. LINNAEA borealis. Fl. lapp. 250. "t. r2. f. 4. Fl. fuec. 562. t. x. Sp.pl. 880 . F. dan. t. 3.

Campanula ferpyllifolia. Baub. prodr. 35. pin. 93.

loc. Succix, prefertim Norlandix, Ruflix, fylva antiqux.

PHARM. LINNAEAE Herba.

Qval fica, [ámaricans.] Rarior, proftans. II: diluens, diuretica, [fubadfringens.]

vis : Rheumatifmus! Scabies!

COMP.

$9 \operatorname{ES} \Lambda-$

440. SCROPHVLARIA aquatica nondum in unfires officinas recepra eft, facile tamen obtinctur, vel ejus loco foi:a fracedeutis fumaniur.

4+1. LWVAEA noudum intravir mofras oficinas, quamyis frequenuhima in noftra terra, et ufu eximia, iplus vaigi eropereutla cunfirmata, 


\section{DID YNAMIA. ANGIOSPERMIA.}

\section{SES A M V M.}

442. SESAMVM orientale foliis ovato - oblongis integris. Hort. cliff. 318. Sp. pl. 883.

Selamum veterum. Baul. pin. 27.

Loc. Malabaria, Aegyptus.

PhARM. SESAMI Sémina.

Qval. oleofa.

Exoleta.

vis: emolliens, obtundens.

vsvs: Marafmus, Dolorofi, Phlogofis, Pruritus.

COMP.

\section{VITEX.}

443. VITEX Agnus caftus foliis digitatis. ferratis, fpicis verticillatis. Sp. pl. 890.

Vitex latiore folio. Baub. pin. 475.

loc. Neapolis, Sicilix paludofa. Arbor, manfueta. pharm. AGNI CASTI Semina.

QVAl. acris, calida, nidorofa. Exoleta, fuperfuca. vis: fubadftringens, antivenerea?

rsvs: Hyfteria, Gonorrhœa.

COMP.

\section{A C $\triangle$ NTHVS.}

444. ACANTHVS mollis foliis finuatis inermibus. Hort. cliff. 326. ups. 181. Sp.pl. 89r.

Acantus fatiwus feu mollis virgilii. Baub. pin. 383. roc. Italia, Sicilia. Perennis, manzueta. PHARM. BRARCAE VRSI Herba.

QVal. fatua, glutinofa.

vis: emolliens, demulcens? paregorica.

vove: Combuntura.

comp. Clyiteres. Herbo $\mathrm{V}$ emollientes.

CLAS:

4it. IRANCA VRSI in nofris officinis male defumitur ab HE. RALLEO Sphondylio, quod vi diverfifima gaudet. 


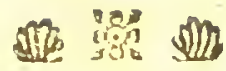

\section{CLASSIS XV.}

\section{TETRADYNAMIA. SILICVLOSA. LEPIDIVM.}

445. LEPIDIVM fativum Horibus tetradynamis, foliis oblongis multifidis. Hort. cliff: $331.4 p 5.183$. Sp. pl. 899.

Nafturtium hortenfe vulgatum. Bauh. pin.103. LOC.

Ammua, cicur. PHARM. NASTVRTII HORTENSIS Herba, Se. mina.

QVAL. acris, orgaftica. Rarior, treftans. v1s: diuretica, mundificans, antifcorbutica.

vsvs: Scorbutus, Hydrops! Afthma, Hernia? Achor, Calculus.

cons.

446. LEPIDIVM Iberis floribus diandris tetrapetalis, foliis inferioribus lanceolatis ferratis, fuperioribus integerrimis. $S p$. pl. 900 .

Ibcris latiore folio. Baub. pin. 97.

Loc. Europa auftralis.

Anmua, cicur.

PHARM. IPERIDIS Herba.

QYAL. acris, orgallica.

vis: incidens, diunetica, mundificans.

vsvs: Febres intermittentes.

COMP.

\section{'THL $\triangle$ S PI.}

447. 'THLASPI arvene filiculis orbiculatis, foliis oblongis dentatis glabris. Fl. Juec. 574. Sp. pl. gor. Fi. dan. t. 793 . 
Thlafpi arvenfe, filiquis latis. Baub. pin. 105.

Loc. Europx agri.

PHARM. THLASPEOS Semina.

Q VAL. dulcis, orgaftica alliata.

Anmua, vulgatiffma.

VIs: pellens, uterina.

vsvs: Rheumatilinus.

COMP. Andromach.

448. THLASPI Burga paftoris filiculis obcordatis, foliis radicalibus pimatifidis, Fl. Sue. 576. Sp. pl. 903. Fl. dan. t. 729.

Burfa paltoris major, folio finuato. Baub. pin. ro8. roc. Europix culta.

Anmun, vulgaris. PHARM. BVRSAE PASTORIS Herba, $\nabla$.

QVAL. aquola. Exoleta, infida.

vis : fubadfringens?

vsve: Hamorrhagia?

COMP.

\section{COCHLEARIA.}

449. COCHLEARIA officinalis foliis radicalibus cordato-fubrotundis, caulinis oblongis. Fl. Juec. 577. Sp. pl. 903. Fl. dan. t. 135 .

Cochlearia folio fubrotundo. Baub. pin. 110.

Loc. Europx littora maritima.

Annua, cicur.

pharm. COCHLEARIAE Herba, Conferva, Syru$p u s, \nabla, \Omega, \circ$.

QVAL. acris, amara, purgans.

vis: diuretica! mundificans, antifcorbutica.

vsvs: Scorbutus! Stomacace, Cachexia, Hydrops,

Hypochondriafis.

conp. Spir. cochlearix purgans.

450. COCHLEARIA Armoracia foliis radicalibus lancenlatis crenatis, caulinis incilis. Fl. fuec. 580. Sp. pl. 904. 
Raphanus rufticanus Baub. pin. $9^{\text {fr. }}$

Loc. Europe autralioris paludes, Perennis, cicur. PHARm. ARMORACIAE Radix, Syrupuis, frigide. Cral. acris. Culinaris, trita, eximia. ris: incidens, ftimulans, diuretica! cofmetica, mundificans, antilcorbutica.

vsvs: Scorbutus, Anorexia, Cachexia, Hydrops! Calculus, Quartana, Cephalalgia, Arthritis. cOMP.

\section{CARDAMINE.}

45r. CARDAMLE praten is foliis pinnatis: foliolis radicalibus fubrotundis, caulinis lanceolatis. $F l$. fuec. 585. Sp. pl: 915 .

Nafturtium pratenie magno flore. Baub. pin. 104. LOC. Europ $x$ palcua aquofa. Perennis, vulgaris. PHARM. CARDAMINES Herba, Flores. QVAL. acriufcula.

vis: antifcorbutica. Fr. antifpafmodica.

vsvs: Ecclampfia.

COMP.

\section{SILIQVOS A. SIS Y MBRIV M.}

452. SISYMPRIVM Nafiurtium filiquis declinatis, fo. liis pinnatis: fololis fubcordatis. Fl. Juec. 592. Sp. pl. gre. Fl din t. 690.

Nalturtium aquaticum fupinum. Barb. pin. 194. Loc. Europa rivuli fontesque. Perennis, infrequens. PHARM. NASTVRTII $A Q V A T I C I$ Herba, Succus $\xi_{v}$, Conjerva, Syrupus, $\nabla, \Omega, \widetilde{V}$.

QV.A.

452. [Cardomine amara Sifymbrio Nafturtio pratantior, ei conn. mode fubßitui poteft, non vero inertior Cardamane pratenfis.] 
I92 TETRADYNAMIA. SILIUVOSA.

QVAL. minus quam Cochlearia acris, orgaflica. Trita, eximia.

vis: diuretica, antifcorbutica.

vsvs: Scorbutus! Obftipatio, Polypus?

COMP.

453. SISYMBRIVM Sopbia petalis calyce minoribus, foliis decompofito - pimnatis. Fl. Juec. 395. Sp. 1). 920. Fl. dan. t. 528 .

Nafturtium fylveftre tenuiffime divilum. Baub. pin. Io5. Loc. Europx macerix tectaque. Annu, vulईaris. PHARM. SOPHIAE Semma.

QVAL. acris, fervida.

Exoletá.

vis: adfringens, anthelninthica.

vsvs: Diarrhoa, Hyfteria, Calculus.

COMP.

\section{ERYSIMVM.}

454. ERYSIMVM officinale filiquis fpica appreffie, foliis runcinatis. Fl. Juec. 598. Sp. pl. 922. Fl. dan. t. 560 .

Eryfimum vulgare. Baub. pin. 100.

loc. Europx ruderata, pagi. Ammua, vulgaris. PHARM. ER YSIMI Herba.

QVAL, acris.

Trita.

vis: abftergens, diuretica.

vsvs: Alhma, Raucedo, Tuffis, Ifchuria.

comp. Syrupus eryfimi.

455. ERYSIMVM Alliaria foliis cordatis. Fl. Juec. 600. Sp. pl. 922 .

Alliaria. Baub. jin. I1о.

Loc. Europe umbrofa.

PHARM. ALLIARIAE. Herba.!

Perernis, vulgaris.

eval. amara, orgaftica alliata. Exoleta, preftans. 
vIS: diuretica, fternutatoria.

vsvs: Afthma, Vlcus fphacelefcens.

COMP.

\section{CHEIRANTHVS.}

456. CHEIRANTHVS Cbeiri foliis lanceolatis acuti. glabris, ramis angulatis, caule fruticofo. Hort. cliff. 334. upf. I87. Sp. pl. 924.

Leucojum luteum vulgare. Baub. pin, 202.

loc. Helvetia, Gallia. Perennis biennisque, manfueta. PHARM. CHEIRI Flores.

CVAL. fragrans, lutea.

Trita. vis: nervina, anodyna, narcotica, pellens, uterina. vsvs: Dolor, Apoplexia, Dyfocia.

COMP.

\section{'B R A S S I C A.}

457. BRASSICA Napus radice caulefcente fufiformis Fl. fuec. 609. Sp. pl. 93 I.

Napus fativa. Bauh. pin. 95

LOC. Europa auftralior, Gothlandia. Biennis, cibur. PHAR. NAPI Semina, $(0)$.

QVAL.

Exoleta.

vis :

vSVS:

COMP.

458. PRASSICA Rapa radice caulefente orbiculari deprefla carnofa. Hort. cliff.339. ups. 190. Sp. pl. 931.

Rapa fativa rotunda. Baub. pin. 89.

LOC.

Biennis, cicur.

PHARM. RAPAE Syrupus frigide.

QVAr. aquofa, efculenta. Culinaris, eximia. vis: diluens, flatulenta, diurctica, aplurodifiaca. 
194 TETRADYNAMIA. SILIQVOSA.

vsvs: Aphtha, Pernio, Afthma, Tufis, Otalgia, Stranguria, Calculus! Colica. COMP.

459. BRASSICA oleracea radice caulefcente tereti car. nofa. Hort. cliff. 338. upp. 189. Sp. pl. 932.

sraflica capitata rubra. Bauh. pir. in. LOC.

Biemis . cicur.

PHARM. BRASSICAE RVBRAE Semina.

QVAl. aquofa, inodora. Exoleta, Juperfura. vis: Hatulenta, alcalefcens.

vsvs: Scorbutus, Raucedo! Cephalalgia crapulce, Combuftura!

COMP.

460. BRASSICA Eruca foliis lyratis, caule hirfuto, filiquis glabris. Hort. up. igo. Sp. pl. 932.

Eruca Latifolia alba. Baub. pin. 98.

Loc. Helvetia.

Annua, cicur.

Pharm. ERVCAE Semina.

QVAL. acris, fervida, penetrans, orgaftica. Exoleta. vis: apophlegmatizans, aphrodifiaca.

vsvs: Apoplexia, Paralyfis lingux, Atecnia.

comr. Conf. diafatyr. Tabule magnaminitatis.

\section{SINAPIS.}

461. SINAPIS nigra filiquis glabris racemo appreflis. Fl. Juec. 61r. Sp. pl.933.

Sinapi rapi folio. Baub. pin. 99.

LOC. Europa.

pharm. SINAlEOS Semina, (1).

Annua, cicur.

eval. acris, orgaftica. Culinaris, trita, praftans.

VIS :

46r. [SINAPIOS femina e Sin. alba xque commode colligi poffunt.] 


\section{TETRADYNAMIA. SILIQVOSA. I95}

vis: incidens, rubefaciens, ftimulans, diuretica, aphroditiaca, antificorbutica.

vsvs: Naulea, Cachexia, Lethargus, Tuflis, Cal. culus, Tinea, Scorbutus.

COMP.

\section{RAPIANVS.}

463. RAPHANVS fativus filiquis teretibus torofis bilocularibus. Hort. cliff.340. upp. 188. Sp. pl. 935. Raphanus major orbicularis vel rotundus. Baub. pin. 96.

LOC.

Annua, cicur. PHARM. RAPHANI Radix (recens), Semina, QVAL. aquofo-acris, orgaftica. Culinaris, exoleta, vis: diluens, attenuans, mundificans, diuretica, carminativa, ructatrix.

vsvs: Althma convulfivum, Raucedo, Ifchuria. rOMP. 
CLASSIS XVI.

\section{MONADELPHIA.}

\section{$D E C A N D R I A$.}

\section{GERANIVM.}

46. GERANIVM mofobatum pedunculis multiforis, floribus pentandris, foliis pinnatis incifis, coryledonibus pimatifidis. Sp.pl.95r. Gacq. bort. x. t. 55 . Geranium, cicuta folio, mofchatum. Babls. pin. 319 . цoc. Europa auftralior. Anmu, cicur. PHARM. GERANII MOSCHATI Herba.

QVAL. ambrofiaca. Exoleta. vis: anodyna, vulneraria, exanthematica.

vsvs: Dylenteria, Variola, Carcinoma, Tormina. comp.

464. GERANTVM robertianum pedunculis bifloris, calycibus pilofis decemangulatis. Fl. Juec. 6rg. Sp.pl. 955. Fi. dan. t. 694 .

Geranium robertianum. Baub. jin. 319.

roc. Europe rupes. Anmu, vulgaris. PHARM. GERANII ROBERTIANI Herba.

Qval. falla, hircina. Exoleta. vis: anodyna, pellens, repellens, lactifuga, ad. ftringens, vulneraria.

vsvs: Colica, Nephritica, Hyfteria, Profluvia, Pubo, Carcinoma, Vulnera, Hamaturia, Vlcus vulva mammaque, Rhagas.

cons. 
MONADELPHIA. POLYANDRIA. I97

\section{POLYANDRIA.}

\section{ALTHAEA.}

465. NLTHAEA afficinalis foliis fimplicibus tomentolis. Hout. clify. 343. ups. 204. Sp. pl. 966. Fl. dan. t. 530.

Althe diolcoridis et plinii. Baub. pin. 315 .

use. Gemanix; Belgii, Gallix, Anglix humida.

Perennis, cicur.

PHAr.r. ALTHAEAE Radix, preparata, Mucilago, Herba.

cys. inipida, mucilaginofa.

Trita, eximia.

- Vis: culliens, invilians, nutriens, lubricans.

vis: Dolor, Pleurits, Nephritis, Ifchuria, Calculus, 'Tullis, Raucedo, "Tenefmus.

coxp. I'ngu. althxi, Syr. althxx, Empl. diachyton limplex, Empl. diachylon compof. Empl. meilori, Loboc Althax, Pafta Althax, CataIiámata enollientia et maturantia.

\section{$\Lambda \mathrm{LCE} \Lambda$.}

465. ALCEA rofer folis finuato - angulofis. Hort. cliff. $=43.0 \% \% .204$. Sp.pl. g65.

Matra "ilea tolio lubroundo. Baub. pin. 315.

I.OC. Biennis, cicur.

PHARM. MAIVAE ARDOREAE Flores, Semina. MAr. fatua, mucilaginofa. Trita, fuperflua.

ves: cmolliens, invicans.

isis: Angina, Inliammatio faucium.

COSP

\section{MAL V A.}

467. MLIVA rotundifolin caule proftrato, foliis cordato-orbiculatis obfolete quinquclobatis, pedun- 
198 MONADELPHIA. POLYANDRIA.

culis fructiferis declinatis. Fl. Suec. 626. Sp. pl. 969. Fl. dam. t. 721 .

Malva fylveftris, folio fubrotundo. Baub. pin. 314. Loc. Europa ruderata, platex. Anmua, vulgaris. PHARM. MIAIVAE VVLGARIS Radix, Herba, $\nabla$. Qvar. fatua, mucilaginola. Trita, preftans. vis: emolliens, invilicans, lubricans, laxans. vsvs: Phlogillici, Dolores, Stranguria! comp. Syr. Hyllopi, Syr. Althax, Clyfieres.

\section{G OS SY PIVM.}

408. GOSSYPYVM berbacen foliis quinquefidis fubtus eglandulofis, caule herbaceo. 0 p. pl. 975 .

Gollypium frutefeens, femine albo. Baub. pin. 430. roc. India.

pHARM. GOSSYHI Semina. BOMBACIS Lana.

QVal. fubdulcis.

vis: œconomica.

vsvs: Sem. Nephritis.

comp. Bomb. medicamentis oticis applic.

\section{H IB I S C V S.}

469. HIBISCVS Abelmofcbus foliis fubpeltato-cordais feptemangularibus ferratis, caule hifpido. Fl. zeyl. 261. Hurt up. 206. Sp. pl. 980.

Alcea regyptiaca villofa. Bauh. pin. 317.

Loc. Afia et America calidior.

PHARM. ABEL MíOSCHI Semina.

CVAL. ambrofiaca.

Exoleta, eximia.

vis: exanthematica.

vsus: Contagium, Tinea, Petechia.

COMP. 


\section{औरते \\ CLASSIS XVII.}

D I A D E L P H I A. $H E X A N D R I A$. FVMARIA.

470. FVMARIA bulbofa caule fimplici, bracteis longitudine florum. Fl. flsec. 63r. Sp.pl. 983. Fl. dan. t. 605 .

Fumaria bulbofa, radice cava, major. Banh. pin. 143. Loc. Europa auftralior. Perentivis, cicur. PHARM. ARISTOLOCHIAE FAPACEAE Radix. RYAL. amara, acris, lequente debilior. Exoleta, dubja. vis: pelleits, emmenagoga.

vsvs: Sarcoma.

comp.

471. FVMARIA officinalis pericarpiis monofpermis racemolis, caule diffufo. Fl. Juec.630. Sp.pl.984. Fumaria officinarum et diolcoridis. Baub. pin. 143. 1.oc. Europre agri, culta. Annua, vulgaris. PhARm. FVMARIAE Herba, Conferva, Syrupus, Elfentia, Extractum, $\nabla$, Semina. QVAL inodora, amarifimi.

$v$ is: balfamica, tonica, antacida, corroborans, pellerus, vifceralis, eccoprotica.

vsvs: Scorbutus, Cachexia, Hypochondriafis! Men lancholia, Arthritis, Podagra, Scabies!

comp. Conf. hamech.

\section{OCTANDRIA. POLYGAL $\Lambda$.}

472. POI,YGALA Senega lloribus imberbibus fpicatis, caule erecto herbaces funplicillimo, folis lato- 
lanceolatis. Amæn. acad. 2.p. 126.t.2. Sp.pl. 990. Mill. dict trb. .

Polygala marilandica, caule non ramofo, fpica in faftigio fingulari gracili e Hofculis albis compofita. Raj. $a p p .670$.

coc. Virginia, Penfylvania, Perennis, peregrina. PHARM. SENEGAE Radix.

QVAL. acido-acris, fubaromatica. Imufitata, beroica. vis: refolvens, fralagoga, fubemetica, purgans, diaphoretica, diuretica, incidens, expectorans, vulneraria.

vsvs: Morfura! Peripneumonia! Pleuritis! Hydrops! Arthritis, Marafmus.

COMP.

\section{$D E C A N D R I A$. PTEROCARPVS.}

473. PTEROCARPVS Draco foliis pinnatis. Syjt. nat. 13. p.533. Sp.pl.1662. Facqu. brft. 283.t.183. f. 92. Laff. it.266.273. n. 147. 168.

B. Lingoum Rumph. amb. 2. p.205. t.70. Loc. $\alpha$. America meridionalis.

B. India orientalis, Java. Arber, peregrina. PHARM. DRACONIS SANGVIS.

QVAL.

472. SENEGAE radix fpecificum in Phlogefi membraulacea, hinc officinis noltris dignifima.

[SlinEGA NOSTRAS Pha au/fr. prov. 55. eft Polygala amara, cuj.s herba paftim in pharmacopoliis proftat $t$ in morbis pectoris cuin fructu adhibetur.]

473. SANGVIS DRACONIS a variis arborum freciebus, fuccum rubrum Aillantibus, obtinetur, qux nondam omnes notxe funt. Sujpl pl. [In peninfula Malacca copiofe colligitur a fpecie Calami, et in Clinam tranfportatur, ubi cumlucro venditur. Sang Drag. Socotrinenfem al cadem planta colligi, dubium eft. Malaccenfis fxpe Gummi Dammar, quod Animes eft fpecies, adulteratur, Konig. Retz. fafc. 4. p.5. Sanguinem Draconis Dracasa Draco etiam profert. Fassdell. Dracas. 8.] 
QVAL. refinola, friabilis, ftyptica, inodora, ru. bra.

vis: incraflans, adfringens!

vsvs: Prolluvia, Diarrhøa, Dyfenteria, Stomacace. comp. Empl. Aypticum, Pil. Helvetii.

474. PTEROCARPVS Santalinus foliis ternatis fub: rotundis retufis glaberrimis, petalis crenatis undulatis. Suppl.pl. 318.

Santalum rubrum. Baub. pin. 363 .

Loc. Indire orientalis montes.

Arbor, peregrina. PHARM. SANTALI RVBRI Lignum.

QFA. Lign. faturate rùbrum venis atris, ponderofum. vis : tinctoria.

vsvs:

comp. Spec, et Conf. de Hyacinth. Empl. San. talin. Spec. diatrion Santal.

\section{GENISTA.}

475. GENISTA canarien/is foliis ternatis utrinque pubefcentibus, ramis angulatis. Hort. cliff. 355. Sp. pl. 997.?

Cytifus canarienfis fempervirens et incanus. Comm. bort. 2. p. 103. t. 52.?

roc. Antillx, et Canarix Infuix.

Arbor.

PHARM. RHODII Lignum, OO.

cyar. dura, fubflevcicens, tortuofa, refinofa, fragrans, rolea.

vis: diuretica, fudorifera.

Rarior, prefans.

vsvs:

comp. Odoramenta, f flemutatorius, Balf. apopl.

$$
\text { N } 3
$$

476.

474. Succus dat quoquo fpecien SANGVINIS DRACONIS.

475. RHODil Lignum unde delinnatur non certo liquet. Plurimi ex preditax Genifte radice defumi antumant, flod ex analogia non facile crederem. [Convolvato feopario Stspl, pl. 235. hoc lignum originem debere, non nagis vero finsile ell.] 
476. GJNISTA tinizoria foliis lanceolatis glabris, ramis Ariatis teretibus erectis. Fl. fuec. 634. Sp.pl. 298. Genifta tinctoria germanica. Baub. pin. 395 . roc. Europa aufiralior. Frutex, manfictus. rнakм. GENISTAE Herba, $\theta$; Flores, Semina. QVAL. luteo-tinctoria, infipiad, inodora. Rarior, prostans.

VIs: pellens, purgans.

vsis: Hydrops.

COMP.

\section{ONONIS.}

477. ONONIS Spinofa floribus racemolis geminatis, foliis ternatis: fuperioribus folitariis, ramis $\int_{\mathrm{p}} \mathrm{i}_{\text {- }}$ nofis. Fl. Juec.637. Sp. pl. 1006. n. 2.3. Fl. dan. tab. 783 .

Ononis fpinofa, flore purpureo. Bauls. pin. 389. roc. Europi auftralioris campi. Perennis, cicur. pharm. ONONIDIS Radix, Herba, $\nabla$.

CVAL. ingrata.

'is: diuretica! emmenagoga.

vsis: Hydrocele, Ifchuria, Calculus, Icterus, Sto.

macace, Febris hungarica. [Gonorrhoca.]

comp. Radices V. aperientes minores.

\section{VPIN VS.}

478. LVPINVS allur calycibus alternis inappendicula. tis: labio fuperiore integro, inferiore tridenta. to. Hort will. 209. Sp.pl. 1015.

Lupinus fativus flore albo, Baub. pin. 347.

LOC.

4:6. [GENISTAF: Fierba, Flo Sem. Sal, frequentius a Spartio Pcopario petuntur.] 
DIADELPHIA. DECANDRIA. 203

LOC.

l'HARM. LVPINI Semina.

Annua, cicur.

QYA. ficca, amaricans, ingrata. Exoleta, dubia. VIs: Hatulenta, anthelminthica.

rsvs: Parotis, Scabies.

comp. Farince IV. refolventes. Tracb. myrrhx.

\section{PHASEOL VS.}

479. PHASEOLVS vulgaris caule volubili, foribus racemofis geminis, bracteis calyce minoribus, leguminibus pendulis. Roy. $\operatorname{lug} d \dot{s}$. 367. Hort. up?: 213. Sp. pl, 1016.

Smilax hortenfis 1. Phafeolus major. Baub.pin, 339. IOC. India orientalis.

PHAR M. PHASEOLI Semina.

Qval. ficca, fariacea. Culinaris, exoleta, Juperfua. $v$ is: nutriens, flatulenta, colinetica.

vsvs: Nephritis.

COMP.

\section{DOLICHOS.}

480. DOLICHOS Soja caule erecto flexuofo, race. mis axillaribus erectis, leguminibus pendulis hifpidis fubdifpermis. $F l$ zeyl. 534. Sp. pl. 1023.

Thateolus crectus, filiquis lupini, fructu pifi majoris cardido. Kempl. amem. 837. t.838.

I.OC. India orientalis.

Peregrina

phar.

QW... fubdulcis; ficca, farinacea,

v1: culinaris.

vols: Anorexia.

comp. Intinctus cibi. 


\section{PIS V M.}

481. PISVM fationar petiolis teretibus, flipulis inferne rotundatis crenatis, pedunculis niultiliaris. Sp. pl. 1026.

Pifum arvenfe. Baub. pin. 342.

Loc. Europa agri.

Annua, cicur.

PHARM. PISI Semina.

Qval. dulcis, ficca, farinofa. Culinaris, exoleta. vis: nutriens, flatulenta.

Visve:

COMP.

\section{VICIA.}

482. VICIA Faba caule erecto, petiolis absque cirrhis. Hort. cliff. 369. ups. 218. Sp. pl. 1039.

Faba. Baub. pin. 338 .

Loc. non procul a Mari Cafpio in confiniis Perfir.

Anmu, cicus.

pharm. FABAE Flores, $\nabla$; Semina, Farina, $C i$ neres, $\theta$.

eval. ficca, farinofa. Fl. fragrans. Culinaris, ufitatif. vis:'nutriens, flatulenta, colmetica.

vsvs: Tumor glandularum.

comp. Farince IV refolventes.

\section{ER V VM.}

433. ERVVM Lens pedunculis fubbilloris, feminibus compreflis convexis. Sp.pl. 1039. Fl. dan.t.95. Lens rulgaris. Banb. pin. 06. цoc. Narbona, Galloprovincia. Amnia, cicur. PharM. LENTIVMi Semina.

eval. ficca, farinacea, infipida. Exoleta, dubia. vis: nutriens, flatulenta.

vsvs: Variola?

comp. 
484. ERTVM Ervilia germinibus undato - plicatis, foliis impari - pinnatis. Hort. ups. 224. Sp. pl. I040. Orobus filiquis articulatis, femine majore. Bauh. pin. 346 .

Loc. Genera, Monpelium. Amma, cicur. PHARM. ERVI Semina.

\section{OROBI Farina.}

Qy+z. ficca, farinacca, fubinfipida. Exoleta, infida. vis: maturans.

rsis:

comp. Farince IV refolventes.

\section{I C E R.}

485. CICER arietimm foliolis ferratis. Hort. cliff.370. up. $2 \approx \frac{1}{4}$. Sp. pl. 1040 .

Cicer fativum. Bath. pin. 347 .

roc. Italia, Hifpania.

YHARM. CICERIS Semina alba, rubia.

QV.t. ficca, farinacea, fubnaufeola. Rarior, dubia. vis: nutricns, flatulenta, pellens, litionthriptica vevs: Tumor glandularum.

cosp. Syr. althxx.

\section{GLYCYRRHIZA.}

486. CLYCYRRHIZA glabra leguminibus glabris, fipulis nuilis, foliolo impari petiolato. Hort. cliff: 494. Sp.pl. 1046.

Glucymhiza filiguola vel germanica. Baub. pin. 352 . ror. Narbona, Italia, Hilpania. Perennis, cicur. pHar. I.lUVIRI'TAE Radix, Sucus infpiffatus, Fistractum, Efjentia, Sympus, Baculi, Tro. chifci.

QVAL.

486. [Antiqui utebantur Glycyrrhiza echinatn. Dinfc. Mll. 7. quare noltro tempore in imperio Roflico ad fuccum parandun adhibetur. ] 


\section{DIADELPHIA. DECANDRIA.}

QVAr. mucilaginofa, dulcinima.

Trita, eximia. vis : edulcorans, incraflans, expecturans, anthelminthica.

vsva: Afthma, Nepluritis, Stranguria, Rheuma. tifmus, Tuflis, Arthritis, Phlogiflici.

comp. Syr. glycyrrhizx, Syr. althxa, Andromach. El. diacathol. Spec. diatragacantha, Lob. althxx.

\section{IN DIGOFERA.}

487. INDIGOFERA tinctoria foliis pinnatis obovatis, racemis brevibus, caule fuffruticofo. $S p$. $p l$. ro6r. Fl.:eyl. 273. Hort.up/.208.

Colutex aftinis fruticofa, floribus fpicatis purpureis. Sloan.jam2.2. p.34. t. 179.f. 2. Treav. ebret. t.54.55. toc. India.

Frutex, ferus.

PHARM. INDIGO commune, optimum.

cVal. Succi focculix exficcatx carulex, tinctorix.

Externa.

vis: obftruens! phthiriaca.

vsvs: Diarrhœa, Lochia.

COMP.

\section{G A LEG A.}

488. GALEGA officinalis leguminibns Arictis erectis, foliolis lanceolatis ftriatis nudis. Sp. pl. 1062.

Galega vulgaris. Baub. pin. $35^{2}$.

loc. Hifpania, Italia.

pharm. GALFGAE Herba.

QYíl. fubaromatica.

Pcrennis, Jubcicur.

vis: fudorifera.

vsvs: Peftis? Petechia? Morfura?

COMP.

Rarior, dubia.

A S T R $\Lambda$

48\%. [INDIGO americanum plerumque ex Indigofora argenton paratur, onisfitale tiniloria.] 


\section{ASTRAGALVS.}

489. $\triangle$ STRAGALVS Tragacantha caudice arborefcente, petiolis fpinefcentibus. "P.plo rom3.

Aftragalus aculeatus fruticolis mallicenfis. Pluk. alin. no.

roc. Gallia aufralior, Sicilia, Olympus.

Frutex, manfuctus. PHARM. TRAGACANTHAE Gumi album, nigrum.

QYAL. mucilaginola.

VIS : Lubricans, demulcens, obruindens.

vsvs: Tullis, Raucedo, Nephritis, Stranguria, Colica, comp. Species diatragacanthx, Rotule diatragacanthx, Trocb. alhandali, Trocb. alkekeng.

\section{TR IF OLIVM.}

490. TRIFOLIVM Melilotus officinalis leguminibus racemoris nudis difpermis rugofis acutis, caulo erecto. Fl. Juec. 663. Sp. pl. 1078.

๙. Melilotus officinarum germanix. Baub. pin. 33 r.

B. Melilotus officinarum germanix, flore albo. Bat b. pin. 33 I.

roc. Europa auftalior. PHARM. MELILO'TI ALPI Flores, Herba. CITRINI Flores, Semina.

Qval. fragrans, gravis, mucilaginofa. 'Lita, prefíans. vis: refolicus, emolliens, lubricans.

vsvs: Dyluria, leucorrha.

comp. Eimpl. meiloti.

489 [TRAGACANTHAM, plurimam, fi non omnem quis it: if-

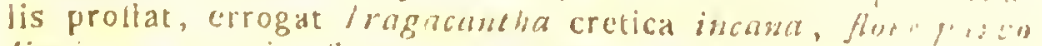
lineis purpureis flriato. lourmef. cor. 29, "10\%. J. , . . 1. 64. Gicoffr. mat. med. x. p. 578.] 
491. TRIFOLIVM repens capitulis umbellaribus, leguminibus tetrafpermis, caule repente. Fl. Juec. 665. Sp. pl. 1080.

Trifolium pratenle album Buub. pin. 327.

toc. Europx pafcua. Perennis, vulgatifima. PHARM. TRIFOLII ALBI Flores.

CVAL. fuaveolens.

Rarior.

VIS:

vsvs: Arthritis.

COMP.

\section{TRIGONELLA.}

492. TRIGONELLA Fonum grecum leguminibus feffilibus frictis erectiufculis fubfalcatis acuminatis, caule erecto. Hort. up. 229. Sp. pl. 1095.

Fœnum græcum fativum. Baub. pin. 348.

coc. Monfpelium. Amma, ciour. pharm. FOENV GR AECI Semina, (D).

evar. fragrans, mucilaginola. Trita, preftans. vis: emolliens, invifcans, lubricans.

vsvs: Ophthalmia, Dyfenteria, Diarrhcea.

comp. Empl. meliloti, Empl. Diachyl. fimpl. Empl.

Diach. comp. Vng\%. althæx, Lob. fanum, Syr. prafii, Enemata Hippiatris. 


\section{政哭 \\ CLASSIS XVIII.}

\section{POL Y A DELPHIA.}

\section{PENTANDRIA. THEOBROMA.}

493. THEORROMA Cacao foliis integerrimis. Hort. cliff. 379. Amen. acad. 7. p. 256. Sp. pl. 1100. facq. obf.r. p. 2.

Cacao. Catesb. car. 3.t. 6.p.6. Merian. Jui. p. 26. t. 26.63. [Somerat. voy. p. ror. $t$ 6r.] qoc. America merid. uliginofa. Arbor, peregrina. PHARM. CACAO Fructus, (D) (Butyrum). QVAl pinguis, fubamara. Culluaris, preftans. vis: nutriens, aphrodifiaca, calefaciens to/t.

vsve: Tabes, Hypochondriafis, Hxmorrhois, (D). Rhagas.

comp. Chocolata.

\section{ICOSANDRIA. CITRVS.}

494. CITRVS Medica petiolis linearibus. Hort. cliff. 379. up $\int .236 . S p . \mu l .1100$.

a. Malus inedica. Baub. pin. 435.

loc. Media, Perfia.

Arbor, manfueta. REARM. CITRI Flavedo, Effentia de cedro, Cor-

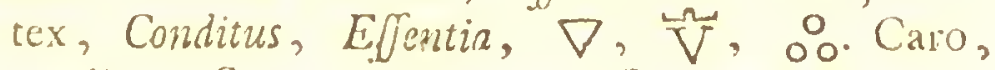
condita, Succus, Conferva, Syrupus. Semina, Florcs.

QVaL. Flnv. aromatica, odorata veficulis oleofis. Cort. alba obflipans, tonica. Pulp. acida. Sen. 2uzara. Fl. fragrans. Trita, optima. 
vis : Flav. cardiaca, pellens, flomachica. Pulp. refrigerans, diurctica. Sem. anthelminthica. $I \%$ analeptica.

rsvs: Pulp. Putridi, Phlogiflici, Sitis, Scorbutüs, Raucedo.

conp. Acet. prophyl.

B. Malus Limonia acida. Baub. pin. 436 .

r.oc. India.

PII.IRM. LIMONIORVM Succus.

QYAL. citro acidior.

vis: citro magis refrigerans.

vsvs: Calculus?

COMP.

495. CITRVS Aurnatium petiolis alatis, foliis acumni. uatis. Hort. cliff. 379. up 5.236. Sp. pl. 1100.

Malus Arantia major. Baneb. pin. 436.

1.0c. India Orientalis. Arbor, manfuetr.

PHARM. NAPHAE (Fiores) condite, Conferva, $\nabla, \Omega$. AVRANTII Folia, Poma inmatura, $C_{1} .$. tex, conditus, Symupus, Elientia, $\nabla, V$, $\circ$, [Berganotte] Flavedo, Effentia.

RYAl. amara. Naplo. fragrans.

Trita, eximitr.

vis: tonica, flomachica, corroborans, anthelmin. thica, pellens. Naph. analeptica.

vsvs: Convulfiones, Hylteria, Hypochondiafis, lcterus, Ifchuria, Scorbutus, Hrmorrh. uteri. comp.

\section{POLIANDRIA. MELALEVCA.}

496. MELALEVCA Lencadendra polyadelpha, fuliis alternis lanceolatis fubfalcatis quinquenerviis, $f_{\mathrm{P}}$. ca clongata. Suppl. jta. [Forjt. flor. 218.] 
Arbor alba Cayputi,. Rumph. amb. 2. p.72. t. 16. I 7. f. . .

L. C. India orientalis. PHAR.I. CAJEYVT Oleum gutt. V.

QYAL. fragrantifima.

Arbor, fera.

Eximia. 'is: pellens, carminativa, antifpasmodica, emmenagogì.

vsvs: Colica flatulenta, Dyfinenorrhce, Morbi Paralytici, Varix ani, Odontalgia.

COMP.

\section{HYPERICVM.}

49?. HYPERTCVM perforction floribus trigynis, caulc ancipiti, foliis obtufis pellucido-punctatis. Fl. fisec. 680. Sp. pl. 1105. Alman. acad. 8. p. 313.

Hypericun vulgare. Baub. pin. 279 .

roc. Europa colles, dumcta. Peremis, vulgaris. PHARM. FYPJEICI Herba, $\nabla$; flores, ' $\mathrm{R}$, (O);

Semina.

2... balfamica, aromatico-fpirans: Trita, eximia. vis: vulneraria! relolvens, anthelminthica!

vsve: Hæmoptyfis, Hxmaturia, Hypochondriafis,

Hyferia, Arthitis, Rhemnatifnus, Phthifis. conp:

498. HYPERICVM bacciferwim floribus pentagynis? corollis barbatis, calycibus lavibus, foliis ovatis; caule fruticofo. Suppl.344. Mant.2. p.277.

Hypericum guianende. Aubl. guian. 784. t. 3 II.

Caa-opia, MIarcgr, braf.96: Pif. brafil.6i.

LoC. Surinama.
PHARM. GVTTAE GVMMì AMERIC Arbor, f

Qval. lutea.

Gallis ujitatum.

VIS:

vsvs: Serpigo:

497 [Hyjpericum quadraingulzum prafert Bergius mat. mod.399.] 


\section{CLA S I S XIX.}

S Y N GEN E S I A.

\section{POLIGAMIA AEQVALIS.}

\section{TRAGOPOGON.}

499. TR $\Lambda$ GOPOGON pratenfe calycibus corollix ra. dium rquantibus, foliis integris flrictis. $1 \%$. Juec. 684. Sp. pl. Irog.

Tragopogon pratenfe luteun majus. Baub. pin.274. Loc. Europx prata.

PHARM. BARBAE HIRCI Radix,

Qval. lactefcens, amaricans. Callinaris, exoleta. vis: nutriens.

vsvs: Tullis, Stranguria.

COAP.

\section{SCORZONERA.}

500. SCORZONERA bumilis caule fubnudo unilloro; foliis lato-lanceolatis nervolis planis. Fl. fuec. 685. Sp. pl. 1112. Facq. auftr. I. t. 35. Fl. dan t. 816. Scorzonera latifolia humilis nervofa. Bauh. pin. 275. LOC. Europie prata.

Perennis, anulgaris. PHARM. SCORZONERAE Radix, condita, $\nabla$; Semina.

QVAL. amaricans, Latefcens.

vis: alexiteria?

vsvs: Morfura? Venenum?

COMP.

Trita, infida.

LACTV.

500. [SCORZONERAE rad. et fem. e Scorzonera hifpanica Officine plerxeque colligun, et in Pharm. Suec, aliisque colligere jubentur. Linnews humilem, ut indigenam et viribus fuperiorem, fubftitni maluit.] 
SINGENESIA. POLYGAMIA AEQVALIS. $21 \%$

\section{A C T V CA.}

5or. LACTVCA fativa foliis rotundatis: caulinis cordatis, caule corymbolo. Sp.pl. mis.

Laciea. Baub. pin. IIs.

r.oc. Europa.

Annua, cicur. PHARM. LACTVCAE Herba condita hifpanica, $\nabla$; Semina.

Q'al. aquofa oleracea, Iactefcens. Rarior, fuperfua. VIs: refrigerans, diluens, humectans, Lactifera, paregorica, antivenerca.

vsis: Hypochondriafis, Satyriafis.

conp. Vugu. populeum.

502. LAC'T'VCA viro/a foliis horizontalibus carina aculeatis dentatis. $S p . p l$. Ing.

Lactuca fylveftris, odore virolo. Baub. pin. 123. Morif. bift. 3. p. 58. S. 7. t. 3. f. 6 .

1.oc. Europic auftralis aggeres, Biennis, cicur. rHARM, LACTVCAE VIROSAE Herba.

(1)AL. amara, tetra.

vis: anodyna, bypnotica, antifpafmodica, repel. lens, ballamica, tonica.

vsvs: Ilydrops.

COSP.

\section{LEONTODON.}

503. I.EONTODON Taraxacum calyce fquamis inferne reilexis, foliis runcinatis denticulatis $1 x$ vibus. Fl. Juec.693. Sp. pl. 1122. Fil. dan.t.574. Dens leonis latiore folio. Baub. pin. x26. uoc. Europx palcua anica. Perennis, vulgaris. pharm. TARAXACI Radix, Herba, $\nabla$.

QVAr. aquofo-amaricans, oleracea, lacteficus.

Cilinaris, ufitata. 
2I SYNGENEEIA. POLYGAMIA AEQVALIS.

vis: faponacea, diluens, humectans, diuretica! vsrs: Hypocinoidriais, Icterus!

cONP.

\section{HIERACIVM.}

504. HIERACIVM Pilofella foliis integerrimis ovatis fubtus tomentotis, folonibus repentibus, fapo unilloro. F\% fucc. 697. Sp. pl. 1i25. Pilofelln major repons hirfuta. Baub. pin. 262. roc. Europie pafua arida. Peremis, frequens. PHARM. AVRICVLAE MVRIS Herba.

Qral. anara, ladelicens.

Exoleta.

V1s: adfringens, vulnoraria.

vsvs: Hernia, Diarrhoca, Scabies, Herpes Stecc. comr.

\section{CICHORIVM.}

505. CICHORIVM Intybus Horibus geminis fetrilibus, foliis rmmeinatis. Fl. finec. 711. Sp.pl. Ir42.

Cichoriun fylveftre f. officinarum. Bath. pin. 125.

Loc. Europa ad margines agrorum viarumque.

Biennis, cicur.

pharm. CICHORII Radix, condita; Herba, Syrupus, $\nabla$; Flores, Conferva; Semina.

avar aquolo-amara, Havo-tinctoria. Trita. vis: aperiens, tonica, diuretica.

vsvs: Mulancholia, Hypochondriafis, Hectica, Hxmorhagia, Podagra.

comp. Sem. IV trigida minora; Syr. cichorii cum rheo.

506. CICHORIVM Endivia floribus folitariis pedunculatis, foliis integris crènatis. Hort. cirfy. 389. upS. $247 . S_{p} . p l .1142$. 
SYNGENESIA. POLYGAMIA AEQVALIS. 215

Intybus lativa latifolia, five Endivia vulgáris. Baub. pin. 125 .

r.oc. Europra.

Biennis, cicur.

THARH. ENDIVIAE Herba, Syrupus, $\nabla$; Semina. QVAL. oleracea.

vrs: aperiens, duetica, mundificans, eccoprotica, vis: Icterus.

comp. Semina IV frigid. minora.

\section{$\triangle R C T I V M$}

50\%. ARCTIVM Lappa foliis cordatis inermibus petiolatis. Fl. fuec.712. Sp. pll.1143. Fl. dan. t. 642. Lappa major f. Arclium diofcoridis. Baub. pin. Ig8. Loc. Europe incarcerata. Peremis, vulgaris. PHARM. BARDANAE Radix, Herba, Semina. QVAL. dulci - fubauftera, urinaria alba, crepitans. Sem. amarifima. Trita. VIs: efculcrita, diuretica, mundificans. Sem. purgans. vsvs: Phlogofis, Nephritis, Arthritis, Syphilis, Oedema.

comp. Ed]. bardanx compofita.

\section{CARDVVS.}

508. CARDVVS mariamus foliis amplexicalibus hafiatopinnatifidis fpinofis, calycibus aphyllis, fpinis canaliculatis duplicato- Spinofis. Sp. $/$. Ins3.

Cardus albis maculis notatus vulgaris. Liuntu. pin. $38 \mathrm{I}$. roc. Italix, Gallix, Anglix aggeres. Anma, cir ri. PHARM. CARDVI MARIAE Herba, $\nabla$; Semina. QVAT. amara.

VIs: thoracica, fudorifera, diuretica $\int e m$.

vess: Pleuriti, Leucorhac? Hydrophubia? Car. cinoma.

Cons.

$\mathrm{O}_{4} \quad \mathrm{C} i \mathrm{R}$. 
216 SYNGENESIA. POLYGAMIA AEQVALIS.

\section{CARLINA.}

509. CARLINA acaulis caule unifloro flore breviore. Hort. cliff.395. upl: 252. Sp. pl. 1160.

Carlina acaulos, magno ilore albo. Buub. pin.380. soc. Italix, Germanix montola. Perennis, cicur. PHARM. CARLINAE (CARDOPATIAE) Radix. QYAI. graveolens, acris, amara, aromatica. Rarior. vis: pellens, fudorifera, flomachica. vsis: Hypochondriafis, Hylteria! Scabics. comp. Orvietanum, t theriacale.

\section{CARTHAMVS.}

510. CARTHAMVS tinctorius foliis ovatis integris ferrato-aculcatis. Hort. cliff.394. ups.251. Sp. pl. II6z.

Cnicus fativus f. Carthamus officinar. Baub. pin. 378 . Loc. Aegyptus.

Exoleta, fubcicur. pharm. CARThami Flores, Semina.

Qvar. naufeofa, lutea.

Rarior. vis: purgans, diuretica.

vsvs: Tufis fenilis, Afthma, Hydrops, Icterus. COMP.

\section{SPIL A NTHVS.}

511. SPILANTHVS Acmella foliis ovatis ferratis, cau. le erecto, horibus radiatis. Sy/t. nat. 12. p.6ro. Verberina Acmella. Sp. pl. I271.

Chryfanthemum bidens f. Bidens zeylanica, flore luteo, lamii folio. Breyn. icon. 48. t. 48 .

Loc. Zeytona.

Annua, peregrina.

PHAKM.

51. ACMELLAE fuccedaneum efro Sigesbertias, Tolanicus intelliget, qua: facile in lobtis noftris luxuriat. [Dians th $i$ partibu et cermsta, an fuccedanea Acmellae?] 


\section{SYNGENESIA. POLYGAMIA AEQVALIS. 2 I 7}

ruarm. ACMELLAE Herba, Semina.

eval. balfanica, amara, fapore odoreque Siges. beckix.

Imufitata, eximia, pretiofa.

Yis: anodyna, attenuans, diaphoretica, diuretica, eminenagoga.

vsvs: Hydrops, Ifchuria, Calculus! Podagra, Leucorrhœa, Pleuritis.

coms.

\section{EVPATORIVM.}

512. EVPATORIVM cannabinm foliis digitatis. F\%. fuec. 728. Sp.pl. 1173. Fl. dan. t. 745 .

Eupatorium cannabinum. Baub. pin. 320.

цос. Europa ripx fluviorum. Perennis, frequens. PHARM. EVPATORII Radix, Herba.

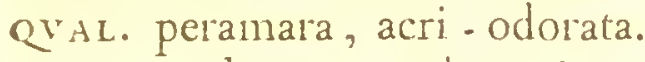

Exoleta.

$v i s$ : corroborans, vulneraria. $R d$. purgans! emeti.

ca! diuretica.

vsvs: Cachexia, Hydrops! Oedema, Hydrocele.

comp.

\section{SANTOLINA.}

513. SANTOLINA Cbancecypariffus pedunculis unifloris, foliis quadrifariam dentatis. Hort. cliff. 397. up/. 252. Sp. pl. 1179.

Abrotanum femina, foliis terctibus. Baub. pins. $1 ; 6$. xoc. Europa auftralis.

PHARM. SANTOLINAE Herba.

RVAL. amara, nidorofa. Rarior, preftans.
VIS: ballamica, tonica, pellens, diaphoretica, carminativa, anthelminthica!

vsvs: Leucorrlica, Plcuritis?

comp. Syr. hystericus.
$\mathrm{O}_{5}$
POL. T. 
218 STNGTNESIA. POLYGAMIA SVPERTT VA.

\section{POLPGAMIA STPERTLVA. TANACETVM.}

514. TANACETVM vulgare foliis bipinnatis incifis ferratis. Fl. flec. 730. Sp. Hlirst. Ml. dan.t.871. a. Tanacetum vilgare lutem. Bahb. pin. 132 . B. Tanacetum folis crifpis. Bauh. pin. I32.

Loc. a. Furopa margines pratorun. Peremis, cicur. pharm. TAN ACETí a. Flores, Semina.

B. Herba; $\nabla, 0^{\circ}$

Qval. amara, nidorofa halituofa, lute - tinctoria. Preflans.

vis: carminativa, pellens, fudonifera! cmmenagroga! antheminthica, antiputredinola, ballamica, tonica, fomachica.

vsva: Tertiana, Hyfleria, Cachexia, Hydrope, I.tumbrici.

cons.

515. TANACETVM Balfamita foliis ovatis integris ferratis. Hort. cliff.389. ups. 255. Sp. pl. 1184. Mentha hortenfis corymbifera. Baub. pin. 226. loc. Hetruria, Narbona. Perennis, cicrer. pHARM. BALSAMITAE Herba.

QYAL. amara, nidorola halituofa. Rarior, prefanis. vis: ftomachica, pellens, emmenagoga.

vsvs: Hyfteria, Melancholia, Opium.

cons?

\section{A R TEMISIA.}

516. ARTEMISIA Contra fruticofa, foliis palmatis linearibus minutis, panicula racemofa, horibus fellilibus. Mant. 282 .

515. [Sem. CINAF, fecundum Pharm. rolf. tam Artesifian Can-

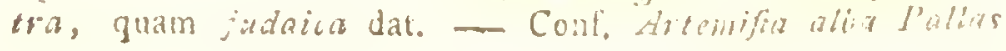




\section{SYNGENESIA. POLYGAMIA SVPERFLVA. 219}

10c. Regnum Boutang Mogolis, Caramania Perfiz, Palaftina per Alexandriain, Arabia, Numidia. pharm. CINAE (SAN'TONIEI) Semina zj, Confeitio.

QVAL. amara.

Trita, preftans. vis: ballamica, tonica, fomachica, anthelminthica. vsrs: Anorevia, Lumbrici.

COMP.

517. ARTEMISIA Abrotanum fruticofa, foliis fetaceis ramofnimis. Hort. cliff. 403. up. 257. Sp. pl. 1135 .

Abrotanum mas angutifolium majus. Baub. pir.136. loc. Sibiria, Cappadocia, Syria, Galatia.

Suffruticofa, cicur.

PHARM. ABROTANI Herba, $\nabla$.

QVAl. amara, nidorofa halituofa. Ravior, preftans. vis: balfamica, antacida, tonica, ftomachica, pellens, emmenagoga, anthelminthica.

vsve: Achor, Tinea, Gangrana, Cachexia. comp. Viggu. martiatum.

518. ARTEMIISIA campeftris foliis multifidis linearibus, caulibus procumbentibus virgatis. Hort. cliff. 403. Fl. Juec. 732. Gmel. fib.2. p. I17.

Abrotanum campeftre. Baub. pins. $1_{3} 6$.

Loc. Europa campi aprici, aridi. Perennis, viligaris. PHARM. ARTEMISIAE CAMPESTRIS Herba, Semina.

RVAL.

is:

risvs: Plcuritis, Vermes. cuins.

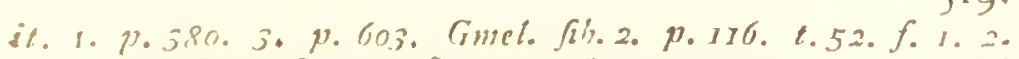

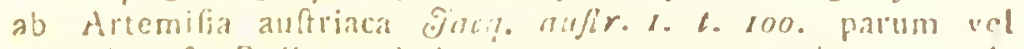

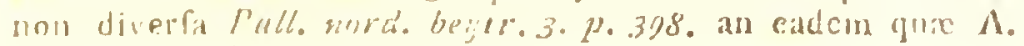
Contra Limn. : ira videtur.] 


\section{SYNGLNESIA. POLYGAMIA SVPERFLVA:}

519. ARTEMIISIA pontica foliis multipartitis fubtus tomentofis, floribus fubrotundis nutantibus: receptaculo nudo. Hort. cliff. 404. ups. 257. Sp. pi. 1187 .

Abfinthium ponticum tenuifolium incanum. Baub. pin. 138 .

luci. Thracia, Myfia, Pannonia, Perennis, cicur. PHARM. ABSINTHII PON'TICT II Qvas. fequenti fuaveolentior. Larior, proflans. vis: fequenti debilior.

vsve: lequenti debilior.

romp.

520. ARTEMISI A Abintbizm foliis compofitis multifidis, furibus fubglobofis pendulis: recepta. culo villofo. Fl. fuec. 735. Sp. pl. 1188 .

Abfintinium ponticum feu romanum ofticinarum feu diofcoridis. Baub. pin. 138 .

Loc. Europx ruderata. Subperennis, vulgaris. WHARM. ABSINTHII VVLGARIS Herba, Syrupus, Conferva, $\nabla, \Omega, \circ$, $(0, \theta$, cry/tall.; $\Theta$ nor cryftall.; Effent. limpl.; Lixtract. in/piflat. Qral. amarifima, nidorofa halituofa, lactaria, urinaria.

Trita, eximia.

vis: balfamica, antacida, antiputredinofa, anthelminthica, Atomachica, antivenerea, antiophthalmica, pellens.

ress: Iliaca, Nephritica, Hydrops, Icterus, Calculus, Iternia, 'Iinea, Achor, Gangrana, Vermies.

comp. El]. abfinthii compofita, Vngu. mattiatum, Conf. hamech, Empl. melilot.

52T, ARTENISIA oulgaris foliis pinnatiflis planis incifis lubtus tomentolis, racemis fimplicibus reculsatis, Horun radio quinquetioro. Sp. plons8. 
a Artemifia vulgaris major. Baub. pin. 137. caule et liore purpuralcente. $T$.

B. Artemifia vulgaris major, caule et flore albicante. Toumlef. inft. 460 .

Lor. Europx culta.

Perennis, vulgatiffma. PHARM, ARTEMISIAE RVBRAE Herba, Syrupus, Elfentia, $\nabla, \Theta$. ALPAE Herba, Moxa, Carbones (radices vetufix).

CVAL. amara. Trita, preeftans. vis: corroborans, analeptica, uterina, pellens, emmenagoga.

vsvs: Tertiana, Dyftocia, Carb. Epilepfra? Mox. Podagra.

comp. Syr. artemilix compolitus. I'ngu. martiat.

\section{GNAPHALIVM.}

522. GNAPHALIVM arenarium herbaceun, foliis lan. ceolatis: inferioribus obtufis, corymbo compofito, caulibus fimpliciffimis. Fl. fuec 738. Sp. pl. irg5. Fil. dan. t.641.

Elichrytum l. Stoechas citrina latifolia. Baub.pin.264. s.oc. Furopa auftralior. Perennis, cicur. PHARM. S'TOECHADIS CITRINAE Flores.

Qval. llava, ficca.

Inufitata.

vis: exficcans.

rsvs: Arthritis.

comp. Suffunigia.

523. GNAPHNAIVM divicum farmentis procumbentibus, caule fimplicilimo, corymbo limplici, tio-

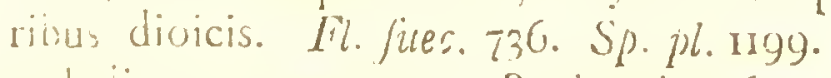

Gnaphatiun montanum. Bauh. fin. 263.

roc. Europx campi exaridi. Perennis, frequens. 
222 SYNGENESIA. FOLYGAMIA SVPERFLVA.

PHARM GNAPHALII Filores.

Qyal. ficca.

Exoleta, infida.

'Is: exficcans, adtringens, roborans.

rsve: Arthritis? Tuhis convulfiva? Diarrhoca.

COMP:

\section{TVSSSILAG O.}

524. TVSSILAGO Furfara fapo unifloro imbricato, foliis lubcordatis angulatis denticulatis. Fl. Jitec. 743. Sp. pl. 1214. It. dan.'t. 595.

Tullilago vulgaris: Baub. pin. 197.

s.oc. Europx fracta argillof. Pcremis, vulgaris. IHARM. FARFARAE Radir, Herba.

TVSSILAGINIS Flores, Conferva, Syrupus, Loboc.

Nor. amara.

Trita.

is: thoracica.

rsvs: Tuflis, Afthma, Phthifis?

COMI.

525. TVSSLago Petafites thyrfo ovato, flofculis fe: mineis nudis paucis. Fl. Juec. 746. Sp. pl. 1215. Fl. dan. 1. 842 .

Petafites major et vulgaris. Bauls. tin. 197 .

roc. Germana, Anglia, Gallia, Peromis, cicar. PHARM. PETASITIDIS Radix, Flores.

QVAL. amara.

Rarior.

vis: fudorifera, alexiteria.

vsvs: Peftis.

comp. + prophylacticum.

\section{SOLIDAGO.}

526. SOLIDAGO Virga aurea caule fubflexuofo an: gulato, racemis paniculatis erectis confertis. $F l$. juec. 754. Sp.pl.1235: Fl. dan. t. 663. 
Virga aurea latifolia ferrata. Baub. pin. 268.

loc Europe prata lylatica. Perentis, oulgaris. FHAKM. VIRGAE AVREAE Her'a.

RYAL. balfamica.

is: vulncraria, diuretica.

$V$ fitata:

vsvs: Calculus, Vlcus.

COMP.

\section{N V L A.}

527. INVLA Helenimm folnis amplexicaulibus ovatis rugolis fubtus tomentofis, calycum jquamis ovatis. Fl. fuec. 755 . Sp. pl. 1236. Ll. dan. 6. 728 . Helenium vulgare. Baub. pin. 216.

Loc. Europa auftralior. Peremis, cicur. Iharir. ENVLAE Radix, condita, Conferva, EffSentia, Extraitum, $\nabla$.

Qral. aromatica, mucilaginofa. Irita, preftans. vis: alexiteria, pellens, expectorans, ftomachica, anthelminthica.

vas: Tuflis, Alhma humidum, Hypochondria. fis, Colica, Tremor, Scabies!

CONH.

523. INVLA dyfenterica foliis amplexicaulibus cordatooblongis, caule villofo paniculato, fquanis calycinis fetaceis. Fl. fuec. 757. Sp. pl. 1237. Fl. dan. t. 4 Io.

Conyza media afteris flore luteo, $f$. tertia diofcoridis. Baub. pin. 265.

Loc. Europa humida.

Perennis.

PHARM. CONYZAE Herba.

Qyat. [acris, lubaromatica.]

V1s: [tomica.]

vsvs: Dyfenteria! Scabies.

CUO.MP. 


\section{AR NICA.}

529. ARNICA montan luliis ovatis integris, caulinis geminis oppofitis. Fil.juec. 760. Sp. pl. 1245 . Fi. dan, t. 63.

Dorouicum plantaginis folio, alterum. Bath. pin. 185. Loc. Europa aufralion, Alpes. Perennis, cicur. PHARM. ARNicAE Terba, Flores.

QVAL. virola, naufeola. Trita, beroica. vis: refolvens, vulueraria, narcotica, vomitoria, purgans, diuretica, emmenagoga, errhina, repellens.

vsvs: Epilepria, Sugillatio, Calus ab alto, [Para. lyfis, Amaurolis, Uuartana.]

COMP.

\section{DORONICVM.}

530. DORONICVM pardalianches foliis cordatis ob. tufis denticulatis radicalibus petiolatis, caulinis amplexicaulibus. Sp. pl. 1247. Facq. auftr. 4.t.350. Doronicum radice fcorpii. Baub. pin. IS4.

i.oc. Apes Vallefix, Cenevx, Thuiri. Peremis, cicur. PHARM. DORONICI Radix.

"QVAI. aromatica, dulcis, fapida. Exoleta, dubia. vis: lubvencmata? Anicx.

vers: Colica, Vertigo?

COMP.

\section{B TiL I. IS.}

531. BEILIS perenzis fcapo nudo. Fi. Suec. $7_{6 \bar{z}}$. Sp: pl.1248. F\% dan. t. 503 .

Betlis fylvefuris minor. Bamb. pin. 26x. s.oc. liuropa palcua aprica.

Perennis, cicur. PHARM.

350. [Var. B. Dornaicum latifolium C"aj. quam icon Jacquim niana exhibet, fumendan efe, monet l'hermgiopara an/ir. prov. p.jol 
PHARM. BELLIDIS MINORIS Flores.

QVAL. inodora, ftyptica. Trita, infida, ftabliana. vis: refolvens? vulneraria, eccoprotica.

vsvs: Hectica? Peripneumonia? Hæmorrhagia? Hypochondrialis?

CO.MP.

\section{CHR Y S N THEMVM.}

532. CHRYSANTHEMVM: Leucanthemum foliis am. plexicaulibus oblongis: fuperne ferratis, inferne dentatis. Fl. Juec. 763. Sp. pl. 1251.

Bellis fylveftris, caule foliofo, major. Baub. tin. 26 r. Loc. Europæ prata. Perennis, frequens. PHARM. BELLIDIS PRATENSIS Herba.

QVAL. fubacris, penetrans, piperata in decocto. Exoleta. vIs: diuretica, vulneraria.

vsve: Phthifis, Afluma, Tinea.

COMP.

\section{MATRICARIA.}

533. MATRICARIA Partbcnium foliis compofitis planis: foliolis ovatis incifis, pedunculis ramofis. Hort. cliff. 415. ups. 263. Sp. pl. 1255. Fl. dan. t. 674 .

Matricaria vulgaris f. fativa. Baub. pin. 133. Loc. Europx auftralioris ruderata. Subperemis, cicur. PHARM. MATRICARIAE Herba, $\nabla$, ○ , Flores. QV.A. amara, nidorola halituola. Vfitata, preftans. vis: anodyna, tonica, ftomachica emmenagoga, Jactifuga.

vsvs: Anorexia, Iliaca infantum, Colica, Hemi. crania, Clavus, Hyfteria!

comp. Syr. Artemifix compofitus, Empl. ranar. mercuriale. 


\section{SYNGFNESIA. POLYGAMIA SWPEREI,VA.}

534 MATRICARIA Cbamomilla receptaculis conicis, radiis patentibus, fquamis calycinis marghine aqua. libus. Fl. Suec. 764. Sp. fl. 1256.

Chamanclums vulgare, Leucanthemum diafcori. dis. Baub. pin. 135 .

roc. Europx agri, ruderata. Ammu, vulgaris. yHARM. CHAMOMILLAE NOSTRATIS Herba, Symupus, $\nabla$, ,, 00 corml. Flores $3 \beta$. cyat. amara, odorata. Litita, preftans. ris: emolliens?

vsus: Tertiana, Cardialgia, Colica, Vomitus gra. vidarum, Calculus, Dyfuria.

comp. Balnea, Pediluvia, Clyfteres, Cataplafmata.

\section{A NTHEMIS.}

535. 'ANTHEMIS nobilis foliis pinnato - compofitis linearibus acutis fubvillofis. Sp. pl. 1260 .

Chamanelum nobile $i$. Leucanthemum odoratius. Baub. pin. 135 .

zoc. Europa paleua aprica. Anmula, cicur, PHAR.r. CHAMOMILLAE ROMANAE Herba, 0 ,

Flores.

cyar. amara, fuaveolens. Trita, eximia. yis: balfunica, paregorica, antifpasmodica, emmenagoga, carminativa, emolliens?

vsvs: Nephritis, Tertiana, Colica, Colica puer. perarum, Hyfteria, Calculus.

comp. Clyjteres emollicites.

536. ANTHEMIS Cotula receptaculis conicis, paleis letaceis, feminibus nudis. Fl. fuec. 767. Sp. pl.126r. Chanmenclunn foticium. Baub. pin. 135 . 1.0c. Europix rix, agri. Anmu, vulgaris. PHARII.

535. Cotula fortida omina eft inter officualia nofira. 
PHARM. COTVLAE FOETIDAE Herba, Flores. CVAL. fœtida, amara. vis: anodyna, pellens, repellens.

Inufitata, eximia. vsvs: Hyfteria! Epilepfia, Hydrops, Scrophula, Althma.

COMP.

53\%. ANTHEMIS Pyrethrum caulibus fumplicibus unifloris decumbentibus, foliis pinnato - multifidis. Hort.cliff.414.upf.263.Sp. pl. 1262. Mill.fig. t.39. Buphthalmum creticum, cotula: facie, flore luteo et albo. Breyn. cent. 150. t. 75 .

Loc. T'unetanum regnum.

Perennis, manfueta. IHARM. PYRETHRI VERI Radix, conditu. CYAL. acris, penetrantillima. Rarior, preftans. vis: incidens, errhina, lialagoga, fophifticans. vsvs: Odontalgia, Paralyfis lingure, Dolor pectoris. comp. Philonium.

\section{A CHILLEA.}

538. ACHILLEA Ageratzm foliis lanceolatis obtufis acute ferratis. Hort. cliff. 413.ups. 265. Sp.pl.1264. Ageratum foliis ferratis. Baub. pin. 221.

LOC. Liburnum, Filorentia, Monlpelium.

PHARM. SGERATI Herba.

Perennis, fubcicus.

CVAl. amara, fuaveolens, aromatica. Exoleta, fuperflua. vis : vifceralis?

vsvs: Vulnus.

comp.

539. ACHIILEA Ptarmica foliis lanccolatis acuminatis argute furratis. $S p$. pl. 1266. Fl. dan. t. 643. $\mathrm{P} 2$

Dracun-

537. [Genunum Pyrethram raro in pharmacopoliis deprehendi* tur; ejus loco plerumque \&ihilloa Prarmicer rad. proftat.] 
Dracunculus pratenfis, ferrato folio. Baub. pin.98. zoc. Europa aufralis. Perennis, cicur. Pharm. P'TARMICAE Radix, Herba, Flores. oval. acris, inodora. Exoleta, Hb. Fl. Juperfiua. vis: fialagoga, errhina, flemutatoria, vulneraria. rsvs: Odontalgia.

COMP.

540. ACHILLEA Millefolizm foliis bipinnatis nudis: laciniis linearibus dentatis, caulibus fuperne fulcatis. Fl. fuec.770. Sp. pl.1267. Fl. dan. t. 737 . Millefolium vulgare album. Bauh. pin. 140.

сос. Europa prata, pafcua. Peremuis, frequens. pharM. MILlEFOLII Herba, Effentia, Extractum,

$\nabla, \stackrel{0}{0}$.

QÝl. amaricans, nidorola. Stabliana, trita. vis: narcotica, inebrians, tonica, lillens evacuationes, vulneraria.

vsvs: Hypochondriafis, Hamorrhois, Hæmopty. fis, Vulnus.

COMP.

\section{POLYGAMIA FRVSTRANEA. CENTAVREA.}

541. CENTAVREA Cyanus calycibus ferratis, foliis linearibus integerrimis: infimis dentatis. Fl. Juec. 776. Sp. pl. 1289 .

Cyanus fegetum (flore cxruleo). Baub. pin. 273. Loc. Europx agri. PHARM. CYANI Flores, $\nabla$. QVAI. criulea.

vis: ophthalmica? Anmua, vulgaris. Exoleta, infida, fuperfuna: 


\section{SYNGENESIA. POI.YGAMIA FRVSTRANEA, 289}

rsvs: tinctorius.

COMP.

542. CENTAVREA Beben calycibus fariofis, 'foliis radicalibus lyratis: lobis oppofitis; caulinis anplexicaulibus. $S p$. pl. I2@2.

Rhaponticoides lutea, foliis inferioribus diffectis, ceteris carthami. Vaill. act. parif. 1718. p. 229.

Loc. Libanus. Perenis, peregrina.

PHARM. BEHEN ALBI Radix.

Qyal. olorata, acris. Exolata, dubia, Juperfun, vis: corroborans, cordialis.

vavs: 'Tremor.

COMP.

5 t3. CENTAVREA benedicta calycibus duplicato. fpinofis lanatis invclucratis, foliis femidecurren. tibus denticulato- Ppinofis. Sp.pl. 1296 .

Carduus fylveftris lirfutus live "Carduus benedictus. Baub. pin. 378 .

zoc. Agri in Lemno, Chio. Annta, cicur. "HARM. CARDVI BENEDIC'TI Herba, Conferva, $\nabla ;+$, Flores, Semina.

evac. amarifima.

Vfitata, preftans. vis: balfamica, antacida, tonica, pellens, fudorifera, diurctica, fubemetica, eccoprotica.

vsve: Anorexia, Cachexia, Arthritis, Vertigo, Hemicrania, Tertiana, Pleuritis, Pellis, Sugillatio.

COMP.

544. CENTAVREA Calcitrapsa calycibus fubduplicato - fpinofis feflilibus, foliis pinnatifidis linearibus dentatis, caule pilofo. Hort. up. 273. "Sp. pl. 1297 . 
230 SYNGENESIA. POLYGAMIA NECESSARIA.

Carduus Acllatus, foliis papaveris eriatici. Barb. pin. 387 .

loc. Germania, Helvetia, Anglia ad vias. Anmu, cicur. PHARM. CALCITRAPAE Radix.

QVAL. amara.

vis: balfamica, tonica.

Infueta.

rsvs: Febres intermittentes!

COMP.

\section{POLYGAMIA NECESSARIA. CALENDVLA.}

545. CALENDVLA officinalis feminibus cymbiformis bus muricatis incurvatis nmibus. Sp. pl. 304. Caltha vulgaris, Baub. pin. 275 .

Loc. Europa auftralis arva.

Amma, cicur. pharn. Calendullae Herba, Flores, Conferva, $\nabla,+$, Semina.

QVAL. amara, fubnarcotica, flava, crepitans.

Trita, preflans. vis: pellens, emmenagoga, fudorifera, exanthematica.

vsvs: Ophthalmia, Vertigo, Febres cxanthematicx. conp.

\section{MONOGAMIA. LOBELIA.}

546. LOBELIA fyplibitica caule erecto, foliis ovato - lanceolatis fublerratis, calycum finubus reflexis.

546. Alia Americanornm contra Syphilitidem fpecifica funt: ECHITES Syphilitica. Infufum. EVPHORBIA canefcars. Decotum. STILLINGiA fyluatica. Flores, etc.

Latefcentes, Campanacex et Coutorte fere omnes ditureticre et mundificantes praftantiores cenfentur lndis; inde centra Syphilikidem laudantur. Sappl. $\mu / .303$. 
SYNGENESIA. MONOGAMIA. 23I

flexis. Hort. cliff. 426. * Ancu. acad. 4. p. $527 . *$ Sp.pi. I320.

Rapunculıs americanus, flore dilute caruleo. Do dart. mem. 297 .

soc. Virginia fylva arid $x$, luto fx. Perennis, manfueta. PHARM. LOBELLAE Radix.

crar. lactelcens, acris, naufeofa.

Eximin.

is: pellens, purgans, ennetica.

vove: Syphilis!

CONP.

\section{VIOLA.}

547. VIOLA odorata acaulis, foliis cordatis, folonibus reptantibus. Fl. fuec. 784. Sp. pl. 1324. Fil. dan. t. 309.

Viola martia purpurea, flore fimplici odoro. Bauh. pin. 199.

Loc. Europa aufralior.

Perennis, cicur. PHARr. VIOLAE Flores (corolla rectius), Conferva, Syrupus, Fulepus, Saccbarum, Mel, (), Semina.

VIOLARIAE Herba.

QVAL. Flor. fragrans.

Trita, preftans. vis: $F l$. anodyna; Hb. emolliens; Rad. purgans? cmetica? Sern. diuretica, emetica; Syrup. refrigerans.

vsvs: Sem. Calculus;] Syr. Raucedo, Tuflis, Ex. antheniata.

Comp. Sem. IV cordial. Encmat.

543. VIOI.A Ipecacumba foliis ovalibus margine fubtusque pilofis. Syft. nat. 13. 11.669. Amon. acad. 8. p. 238 . 
232 SYNGENSIA. MONOGAMIA.

Viola grandiflora, veronicre folio villofo. Barr. requin. I13.*

Pombalia Ipecacuanha. Vandell. fafc. p. 7. t.r. Loc. America meridionalis fylva humidx.

Frutefcens, peregrina. YHARM. IPECACVANHAE ALBAE? Radix Эij. QVAL. inodora, levifime pungens.

vis: emetica, tonica, purgans, fudorifera. vsvs: Febres, Diarrhœa, Dyfenteria, Leucorrhoa, Hxmorrhagia uteri. COMP. 


\section{औล}

\section{CLASSIS XX.}

\section{G Y N A N D R I A. $D I A N D R I A$. ORCHIS.}

549. ORCHIS bifolia bulibis indivifis, nectarii labio lanceolato integerrimo: cornu longinimo, peta. lis patentibus. Fl. fuec. 793. Sp. pl. I331.

Orchis alba bifolia minor, culcari oblongo. Baub. pin. 83 .

s.oc. palcua dura Europx.

Perennis, cicur. PHARM. SATYRII Radix, condita.

UAL. hircina, mucilaginofa.

ris: anodyna, exhilarans, invifcans, obtundens, aphrodifiaca, analeptica.

vsvs: Sterilitas, Atecnia, Tabes, Dyfenteria.

comp. Conf. dialatyr.

550. ORCHIS Mo:io bulbis indivifis, nectarii labio quadrifido crenulato: cornu obtufo adfcendente, petalis obtufis conniventibus. Fl. fuec. 794. Sp. pl. 1333. Fl. dan. t. 457.

Orchis morio mas, foliis non maculatis. Vaill. parif. 151. que Buxb. cent. 3. t.5.

loc. Europx, Orientis fylva. Perennis, cicur. PHARM. SALEP Radix.

QVAr. hircina, mucilaginola.

vIs: invifcans, lubricans, nutriens, aphrodifiaca. vsvs: Atecnia! Dyfenteria, Vlcus vefica, Excoria-

tura inteflinorum.

comp.

550. [SALEP rad. matue hona obsinetur ex Orch, maferta, midisut altisqur. 


\section{EPIDENDRVM.}

551. EPIDENDRVM Vamilla fandens, foliis ovarooblongis nervofis feflilibus caulinis, cirthis lpiralibus. Roy. $\operatorname{lug} d l$. 13. Sp.pl. 1347. Plum. ic. 188. [Aublet. guian. 821. Juppl. 77.]

Vanilla piperis arbori jamaicenfis innafcens. Pluk. alm. 38 I. t. $320 . f$. $\mathrm{x}$.

zoc. Indin utriusque arbores. Parafitica, frutex. PHAKM. VANILLAE Silicua 3 .

QYal, pinguis, ambrofiaca. Rarior, eximia. vs : calefaciens, corroborans, cephalica, diureti.

ca, aphrodifiaca.

vsvs: Melancholia, Atecnia.

comp. Chocolata.

\section{HEXANDRIA. A R I S T O L O C I I A.}

55. ARISTOLOCHIA trilobata foliis trilobis, caule volubili, floribus maximis. Sp.pl. 1;6r. Facq. obf. .. p. 8. t.3. Berg. mat. 448.

Ariftolochia folio hederaceo trifido, flore maximo, radice repente. Barr. equ. I6.

LOC. America meridionalis.

Frutex, ferus. PHARA. ARISTOLOCHIAE TRILOBA'TAE Stipites.

QVAL. aromatica, amaricans, diu durans.

vis: alexiteria, ludorifera.

rsvs: Morfura ferpentum, Febres.

COMP.

553. ARISTOLOCHIA Serpentaria foliis cordato-oblongis planis, caulibus infirmis Hexuolis teretibus, foribus fulitariis. $S p \cdot p l .1363$. 
Arifolochia Pifolochia $\int$ Serpentaria virginiana, caule nodolo. Pluk. alm. 50. t. 148. f.5. Catesb. carol. I. t. 29.

loc. Virginia, Carolina. Perennis, man/ueta. PHARM. SERPENTARIAE VIRGINIANAE Radix 5 ß3. El/entio.

QYAL. acris, hircofa odore valerianx. Trita, eximia. vis: pellens, diaphoretica, exanthemathica, alexiteria, diuretica, anthelminthica.

vsvs: Febres intermittentes, exanthematicx, Morfura.

Comp. fomit. Kent.

554. ARISTOLOCHIA rotundn foliis cordatis fubfefl. libus obtufis, caule infirmo, floribus folitariis. Hort. cliff. 432. Sp. pl. 1364.

Ariftolochia rotunda, flore ex purpùra nigro. Bath. pin. 307.

zoc. Italia, Hifpania, G. Narbonenfis.

Perennis, manfueta. PHARM. ARISTOLOCHIAE ROTVNDNE Radix. QVAL. fœida, aromatica, amara, acris, exurens. Vhitata, proefians. vis : calefaciens, incidens, pellens, vomitoria, diuretica, emmenagoga, abortiva, repellens, vulneraria.

vsvs: Cachexia, Chlorolis! Podagra, Scabies, Fi. Aula, Sarcoma, Cacoëthes.

comp. Theriac alexipharm. Troch. capparidis.

555. ARISTOLOCHIA longa foliis cordatis petiolatis integerrimis obtufufculis, caule infirmo, floribus Colitariis. Sp.pl. 1364 .

Ariftolochia longa vera. Bubl. pin. 307.

noc. Europa auftrais: Perenmis, manfueta. 
236 GYNANDRIA. OCTANDRIA.

PHARM. ARISTOLOCHIAE LONGAE Radix.

QVAL. pracedentis.

vis: prixcedentis.

vsvs: pracedentis.

comp. Andromach. Vingu. apofolor. Eimpl. manus

Dei.

556. ARISTOLOCHIA Clematitis foliis cordatis, caule erecto, fioribus axillaribus confertis. Hort. ups. 279. Sp.pl. 1;64.

Ariftolochia clematitis recta. Baub. pin. 307.

1.oc. Gallia autralis. Perennis, cicur. PHARM. ARISTOLOCHIAE VVLGARIS Radix. CVAL. graveolens, amara, acris.

vis: pellens, fubvomitoria.

vsvs: Cachexia, Podagra, Cacoëthes, Sarcoma.

COMP.

\section{OCTANDRIA. \\ CYTINVS.}

55:- CYTINVS Hypociftis. Sy/t. nat. 12. p. 602.

- Hypociftis omnino lutea f. ochra colore. Cluf. bifp. 143.

soc. Hilpania, Lufitania, Mauritania.

Parafitica Cifti, peregrina. pHarm. HYPOCISTIDIS Succus.

CVAL. acido - ftyptica, nigra.

Rarior.

vis: adfringens.

rsvs: Profluvia.

conp. Andromach. Mithridat. Troch. carabe, Empl.

hernix, Paful. Laxativ. 


\section{POLYANDRIA. A R V M.}

558. ARVM maculatum acaule, foliis haftatị integer: rimis, fpadice clavato. Hort. upf. 279. Sp. pl. I370. Fl. dain. t. 505.

Arum vulgare maculatum et non maculatum. Băb: pin. 195.

Ioc. Germania, Italia. Pereminis, cicur: PHARM. ARI Radix $3 \beta$. Ficulce, Herba.

eval. acris; leptica. Irite, preftans. Hb. Juperfua. $\mathbf{v}_{1 S}$ : calefaciens, incidens, faponacea, expectorans, cofmetica, recens corrodens, rubefaciens, veficans. vsvs: Tertiana, Anorexia, Cephaliea fympathica, Afthma humorale, Obftructio, Cachexia, Chlo. rofis, Phthifis, Contufura.

conp. f benedictus. 


\section{CLASSIS XXI.}

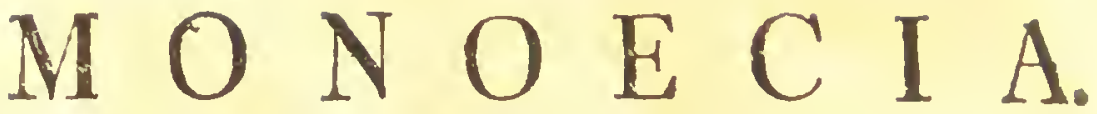

\section{MONANDRIA. \\ CYNOMORIVM.}

559. CYNOMORIVM coccineum. Amcen. acad. 4. p.35r. t. 2. Sp. pl. 1975.

Fungus typhoides coccincus melitenfis. Bocc. fic. 3०. mul:2. p. 69.

Loc. Sicilia, Melita, Mauritania, Jamaica; parafitica radicum Lentifci, Myrti, Halimi, inter

Novembrem et Martium. Herba, peregrina. FHARM. FVNGI MELITENSIS $习 j$.

QVAl, fyptica, nigra.

Eximia.

vis: adfringens, obltipans.

vsvs: Dyfenteria, Vlcus cacoëthicum, Stomaca. ce, Hrmorrhagix.

COMP.

\section{MYRIS TIC A.}

560. MYRISTICA moschata fructibus glabris. Thunb. act. bolm. 1782. p. 46. nov. gen.5. p. 84.

Nux myriftica. Rumph. amb. 2. p. 14. t. 4. 5.

Nux mofchata fructu rotundo. Baril. pin. 407. Pluk. alm. 265. t. 209. f. 1.

Loc. Molucca, prefertim Banda. Arbor, peregrina. PHARM. MOSCHATA (MYRISTICA) Nux, condita, $\nabla, \circ,(\mathbb{D})$, Balfamis.

MACIS, Eljertia, $\nabla, \circ$, (1).

eVAL. Ayptica, fragrans aromatica. Irita, eximia. 


\section{MONOECIA. TRIANDRIA. $\quad .239$}

Vıs: hypnotica, ftomachica, carminativa, obfi. pans, ophthalinica.

vsis: Colica, Diarrhca.

sour. Eilect. diafatyr. Diaphonic. Orvietan. Bened. Empl. ftomachale, Spir. Embryon. Balf. apoplect.

\section{$T R I A N D R I A$. PHYLI,ANTHVS.}

501. PHYLLANTHVS Emblica foliis pinnatis floriferis, caule arboreo, fructu baccato. Fl. zeyl. 333. Sp. pl. 1393.

Nellicamaram. Rbeed. Mal. r. p.6g. t. 38.

Loc. Malabaria, Zeylona.

Arbor, peregrina. PHARM. MYROBALANI EMBLICAE Fructus sii. QVAL. acidiufcula, auftera, fubacris.

vis: adflingens Subft. purgans infuf: Exoleta, Juperfute. vevs: Stomacace, Dylenteria.

coirs. Pil, fine quivus, Conf. hamech.

\section{TETRANDRIA. BET VLA.}

562. PETVLA alba foliis acuminatis ferratis. Fl. Sues. 859. Sp. pl. 1393. [Pall. rofJ. I. p. Gr.] Ketula. Baub. pin. 427.

s.oc. Europx fylvx.

Arbor, frequentifima. YHARM. BETVLAE (epidemidis) o nirrum, Fuligo. Qvi.. fuaveolens 00 .

vis: diaphorctica.

vors: Eryfipelas! [Gonorrhca.]

cosil? 


\section{B VXVS。}

563. BVXVS fempervirens. Hort. up. . 283. Sp. pl.1394. Buxus arborefcens. Baub. pin. 471.

coc Gallia, Helvetia. Arbor, manfueta. PHARM. BVXI Lignum, 0 Gt. XX.

QVAl. tetra fortida, amara. Rarior. vis: fudorifera, mundificans. Fol. purgans, pellens. vsvs: Hirfuties 00 , Epileplia, Hylleria.

COMP.

\section{VRTICA.}

564. VRTIEA pilulifera foliis oppofitis ovatis ferratis, amentis fructiferis globofis. Hort. cliff. 440 . upj. 282. Sp. pl.1395.

Vrtica urens pilulas ferens. Baub. pin. 232.

Loc. G. Narbonenfis, Helvetia, Anglia. Annua, cicur, lHARM. VRTICAE ROMANAE Semina.

QVAL. urens tactu, infipida. Exoleta, fuperfua. vis: diuretica.

vsvs: Rubeola, Nephritis, Hamorrhagia.

comp.!

565, VRTICA dioiva foliis oppofitis cordatis, racemis geminis. Fl. juec. 863. Sp. pl. 1396. Fl. dan.t.746. Vrtica urens maxima. Baub. pin. 232.

Loc. Europx ruderata. Perennis, frequens: PHARM. VRTICAE MAJORIS Radix, Herba, $\nabla$, Semina.

QVAc. urens tactu, fubinfipida, oleracea. Rarior. vis: diuretica, mundificans, ftimulans extus. vsvs: Icterus, Nephritis, Phthifis! Hxmorrhagia, Hxmoptylis, Hxmorrhois, Paralyfis ext. COMP. 
MONOECIA. PENTANDRTA. $24 r$

\section{MOR VS.}

566. MORVS nigra foliis cordatis fcabris. Hurt. cliff. 44.1. up . 283. Sp. pl. I398.

Morus fructu nigro. Baub. pin. 459.

soc. China, Italia.

Arhor, manfueta.

PHARM. MORI Rob, Syrupus, Cortex Rad.

CVal. dulci - fubacida.

Trita, eximia.

vis: refrigerans, corroborans.

vsvs: Febres putridx, Sitis, Angina. Cort. Tænia. comp. Mixture febriles.

\section{PENTANDRIA. XANTHIVM.}

567. XANTHIVM ftrumarium caule inermi, foliis cordatis trinervatis. Fl. ficec. 869. Sp. pl. I400.

Lappa minor 1. Xanthium diofcoridis. Buub. pis.198. loc. Europa auftralion. Anmea, cicur. PHARM. XANTHII [Folia, Semina $3 /$.

Qval. Luteo-tincloria, amara, fubacris. Inufitata. vis: adltringens, pellens, diaphoretica. vsis: [Scrophula, Herpes, Eryfipelas.

conp.

\section{POLYANDRIA. POTERIVM.}

568. POTERIVM Sanmuiforba inerme, canlibus fub. angulolis. IInt. cliff.446. ups. 288. Sp. pl. I4II. Pimpinella Sanguiforba minor hirfuta. Batub. pin $\mathbf{1 6 0 .}$ roc. Europa auftralior. Perentis, cicur. PHARM. PIMIPINELLAE ITTALICAE Herba, Se. mina.

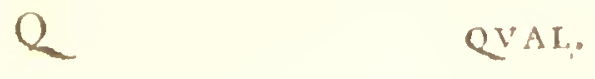


Qvar. Ayptica, fubfragrans.

$v i s:$ adfringens, volneraria.

Culinaris, exoleta, Juperfuce. vsvi: Hramoptyfis? Dyfenteria? camp. Infus. vinola vernal.

\section{QVERCVS.}

569. QVERCVS Suler foliis ovato - oblongis indivilis ferratis fubtus tomentofis, cortice rimofo fungofo. Hort. cliff. 448. Sp. pl. 1413.

Suber latifolium perpetuo virens. Baub. pin. 424. roc. Lufitania, Aquitania, Bxtica. Arbor, manfueta. pHaRM. SVBERIS Cortex.

QVAl. ficca, ftyptica.

VIs: adfiringens, coriaria.

vsus: Dyfenteria, Eryfipelas.

COMT.

570. QVERCVS Robur foliis deciduis oblongis fuper. ne latioribus: finubus acutioribus, angulis ob. tufis. F\%. fuec. 870. Sp. pl. $14 \mathrm{I} 4$.

Quercus cum longo pediculo. Bauh. pin. 420. loc. Europa infra Norlandiam. Arbor, vulgaris: pharm. QVERCVS Contex, $\nabla$, Cupulx, Glandes. Qral. ficca, flyptica. Trita preftans. Vis: adfringens, coriaria, obftipans. vsvs: Diarrhcea, Tertiana, Himorrhagia. comp.

\section{JVGLANS.}

570. JVGLANS regin foliolis ovalibus glabris fubfer. ratis fubrqualibus. Hort. cliff. 449. upf. 286. Sp. 1l. 1415.1

Nux juglans f. regia vulgaris. Baub. pin. 418. 


\section{MONOECIA. POLYANDRIA.}

Ioc. America leptentrionalis? Perfia, Arbor, manfueta. PHARM. JVGLANDIS Nuces, Dillepimentum, $R \ominus b$, (O).

Qral. graveulens, amarigans, denigrans. Nuccl. lubdulcis, ficca.

Ravior, preftrins. vis: narcotica, pellens, emmenagoga, anthelmin. thica, angmans, paralytizans.

vsvs: Diflep. Dyfenteria, VJt. Hremorrhagia, den. tis, (1) cicatrix variolx, Rob. Angina. Nucl. Tænia! comp. Acet. prophylact.

\section{FAGVS.}

572. FAGVS Caftanea foliis lanceolatis acuminato. ferratis fubtus nudis. Hort. cliff. 447. ups. 287. Sp.pl. 1416.

Caftanea. Baub. bift. I. p. I2r.

zoc. Italia, Gallia, Germania. Arbor, manfueta. PHarm. CASTANEAE Fructus, (D), Cortex. QY.A. dulcis, efculenta. Exoleta, imfida, Juperfun. vis: Hatulenta, temulentans, febrilis.

viss: (Q) Excoriatura.

Cons.

533. FAGVS fyluatica foliis ovatis obfolete ferratis. Fi. Suec. $87_{1}$ Sp. pl. 1416.

Fagus, Baub. pin. 419 .

Loc. Europa auttralior. Arbor, cicur. pHarm. FAGI Fructus, (D), Cineres iclavellati. QVAL. infipida. Exoleta, infida, Fr. Juperflua. VIs: temulentans, cephalalgica.

vsves:

Cons.

\section{CORYLVS.}

574. CORYLVVS Avellana fipulis ovatis obtufis. Irt. Juec. 872. Sp.pl.1417. 


\section{MONOECIA. ADELPHIA.}

Corylus fylveftris. Baub. pin. 4 I8.

noc. limopa lepes. Arbor, vuljaris. PHARM. CORYLL Jignum, Oo.

Cval. inodora, infipida, 0 fortida.

vis: $\stackrel{0}{0}$ anodyna. Exoleta, fuperfura.

vsve: ${ }_{0}^{\circ}$ Odontalgia.

COMP.

\section{LIQ VID $\triangle M B A R$.}

575. I.IQVIDAMBAR Styracifua foliis palmato - angulatis: lobis indivifis acutis. Sp pl. Itris.

Liquidambar arbor 1. Atracillua aceris folio, frucu tribuloide. Pluk. alm. 224. t. 42. f.6. Catesb. car. 2. t. 63 .

ı.oc. Virginia, Mexico. Arbor, manfueta. PHARM. STOR ACIS LIQVIDAE Refina.

Qval. Balfaimm liquido-tenax, grifeum, acre, atomaticum, fragrans.

vis: cephalalgica, calefaciens.

vsrs: Scabies.

comp. Vngu. odorata.

\section{ADELPHIA. PINVS.}

576. PINVS fylveftris foliis geminis: primordialibus folitariis glabris. Ho.fuec. 874. Sp.pl.r481. [Pall. r.0.]. 1. p. 5. tab.2.]

Pinus fylveltris. Baub. pin. 491.

Loc. Europx ficca. Arbor, frequentiffma. PHARM. PINI Turiones (male com), Ligni $\Omega$, O Relina, Pix, 응 picis. 


\section{MONOECIA. ADELPHIA.}

TERERINTHINA COMMVNIS, cocta, Cral. refinola.

Vis: diuretica, mundificans.

Trita, preftans.

vsve: Scorbutus.

cons.

577. PINTS Pinea folits geminis: primordialibus folitaris ciliatis. Hort. cliff.450.upf.288. Sp. pl. ratig.

Pinus fativa. Baub. pin. 49I.

Loc. Italia, Galloprovincia, Narbona. Arbor, mand. PHARM. HINI Nuces, nuclei mundati, Loboc.

QMal. dulcis.

is: nutriens, aphrodifiaca.

Trita, preftans.

rsvs: Stranguria, Tabes.

conp. El. diafatyrii.

578. PINVS Larix foliis fafciculatis obtufss. Hort. cliff. 450. Sp. pll. 1420. [Pall. rolJ. I. p. I. t. I.] Larix. Baub. pin. 493.

Loc. Alpes Helveticx alixque, Rufia. Arbor, manfueta. YHARM. 'TERERIN'THLNA VENETA OO, $\Omega$. [I.ARICIS Gummi.]

gvar. refinofa, [interne gummofa.] Trita, preftans.' vis: diuretica unina violacea, vulneraria.

rsve: Phthifis, Jcucorrlica, Vlcera, $\mathrm{O}$ Hamorhagia! Irchuria.

comp. Ingr. digeflivum.

579. PINVS Abies foliis folitariis fubulatis mucronatis levibus bifarian verfis, fil. fuec.875. Sp.pl.142r. Picea major prima f. Abics rubra. Baub. pin. 493. soc. Furnpe humidiufula. Arhor, frequentiflona. pharm. ABIE'I'LS Refina, Colopbuizim. 
QVAL. refinofa, crepitans.

Rarior, preftans.

vis: diuretica.

vsvs: Scorbutus, Arthritis.

COMP.

\section{CVPRESSVS.}

580. CVPRESSVS fempervirens foliis imbricatis, fron. dibus quadrangulis. Hort. cliff. 449. upp. 288. Sp. pl. 1422 .

Cupreflus. Baub. pin. 488.

LOC. Creta.

Arbor, manfucta.

PHARM. CVPRESSI Lignum, Nuces (frobili potius.) QVAT. ficca.

Exoleta, Juperflur.

vis: adtrinerens.

visu: Hernia, Diarrhœa.

C.OMP.

\section{CROTON.}

58. CROTON Ca/carilla foliis lanceolatis acutis in. tegerrimis petiolatis fubtus tomentofis, caule arborco. Amen. acad. 5. p. 4n." Sp. pl. 1424 .

Ricinoides clxagni folio. Catesb. carol.2.p.46.t.46, Plim. ic. 240. f.x.

气oc. l'eru, Florida, Providentia, Paraguay. Arbor, peregrina, FHARA. CASCARILLAE Cortex, Elfentia. QYAY. acris, amara, fragrans, fumo mofchato. Trita, eximia, Jabliana. vis: tonica, fub, eccoprotica, thoracica.

vsvs: Febr. Exacerbantes, Tertiana, Diarrhœa fẹbrilis.

COMP.

582. CRO.

581. Cafcarillom eandem cum THYMIAMATIS reu THVRIS cortice faciunt varii, licet diftinctus in ollicin is notris vendistur. [Anne hic Styracis Spccies? Ph. dan. 33.] 
552. CROTON tinctorium folis rhombeis repandis, caplulis pendulis, caule herbaceo. Hort. up . 290 . Sp.pl.1425. Buml. ind. t.62. f. .

Ricinoides ex qua paratur Tournefol gallorum. Act. Frill. 1712. p. 337. t. I7.

Loc. Narbonic culta.

PHAR.M. LACMVS Succus (Tornejolis).

Qyar. caruleo-tinctoria.

VIS: acidun indicandi.

vsrs: Gangrana, Carcinoma?

conp.

583. CROTON Tiglizm folits ovatis glabris acuminatis ferratis, caule arboreo. Fl.2eyl.343. Sp.pl.1426. Ricinoides indica, folio lucido, fructu glabro. Bum. zeyl.200. t. 90.

Loc. Zeylona.

Anmia, fera.

Exterize.

eharu. TIGLII (TILLI) Grana Gr. iij.

\section{PAVANAE Lignum.}

QYAL, acerrima.

Exoleta, cauta, Juperfuia.

vis: emetica, drafica, exurcns.

vis:

Co)MP.

584. CROTON lacciferum foliis ovatis tomentofis ferru. latis petiolatis, calycibus tomentofis. Jil. zeyl. $344 . *$ Sp.pl. 1426.

Ricinnides aromatica arbor, circxe foliis hivfutis, floribus fpicatis, major. Burm. zeyl.201. t. 9r. r.oc. India.

PHARM. I.ACCAE Gummi (in granis).

Aibor, fera.

$$
\text { Q4 Nat. }
$$

583. PAVANAE lignum in mofiris officinis peregrinum eft.

5\%. GVMIMII LACCAE colligitur circa ramulos ficcos ab irfeais, red quibus? e Croione lacsiforo lacterente decerpmet ha'c infecta fuccum et Laccam fllam inde conficians, icise 
QVAL. Refin. rubra.

vis: lubadfringens, diuretica.

vsve: tinctorius Stomacace.

comp. Vernices, Laccx figillatorix, $\mathrm{R}$.

\section{JATROPHA.}

585. JATROPHA Circas foliis cordatis angulatis. Hort. cliff 445 Sp.pl i429. Gacq bort. 3. t.63.

Ricisuides americana, gollypii folio. Tournef. inft. $165 \%$

ıoc. Surinama, Brafilia, Jamaica. Arbor, fera. PHARM. RICINI MAJORIS SEmina.

QVAl. acris, exurens. Exuleta, cauta, fuperfua. VIs: draflica, inflammatoria.

VSTS:

COMP.

586. JHTROPHA? elnftica foliis ternatis elipticis integerrimis fubtus canis longe petiolatis. Suppl 422.

Hevea guianenlis. Aublet. guian. 871. t.335.

Caoutchouc. ACt. paril. 1751. p.329.t.20. f. 10-I4. 1769. p. 209. Berg. mat. med. 566. Be/cräft. naturf. fr. 3. p. 356 .

Loc. Guiana, Peru.

PHARM. ELASTICA Refina.

Arhor, peregrina.

Qrar. Refinu coriacea, Hexilis, extenfilis, elaftica, fulco-rufefens.

VIS:

VSTS:

Hermanno. [Colligitur a Cocro Lacco, in Ficu religiofa et indica, item in Rlianno Jujuba habitante. Kerr in abt. angl. [1. p. 2.]

586. Granus nondum rite definitum. Succus arboris lacteus in-

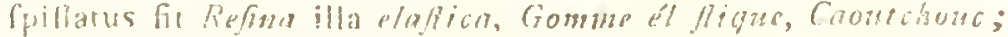
ell ficus insi foropin peltata, aliayur, fimilem fuccum, polt exficcationem clafticum, fundant, Suppl. pl. 
vsrs: mechanicus; chirurgicus.

CUNP.

\section{RICIN VS.}

587. RICINVS communis foliis peltatis fubpalmatis lerratis. Sp pl. r430. Mill. ic. 219.

Ricinus vulgaris. Bab pin 439 .

Loc. temperatum calum IV orbis partum.

Annir, manfueta.

PHARM. RICINI VVLGARIS (CATAPVTIAE MAJORIS) Semina Gr. XXV.

[PALMAE CHRISTI QD.]

QVAL. acris, amara. Sem. oleola. Exoleta, canta. VIE: draftica, inflammatoria, gaftriticans, anthelmintica! [OD laxams.]

vsus: [Ilaca, Colica pictonum, nephritica, Trnia.] COAP.

\section{SINGENESIA. MOMORDICA.}

588. MOMORDICA Elaterum pomis hifpidis, cirrhis nullis, sp pl. I334.

Cucumis ylieftris afininus dictus, Bazth. pin. 314. roc. italid, Nartona, Sicilia. Peremis, nucicur. PHARM. CVCVMERIS ASIMNI Radix Gr.XV-5ß. ELATERII Lixtradum (viride) $\exists j$. Refina (alba) Gr. ij.

QVAL. amarithima, nauleofa. Heroica, canta. vis: enctica, draftica, hydragoga, cmmenagoga, abortiva.

vsis: Hydrops, Mfthma, Icucorrhaca, Coryza, Scrophula.

comp. Lixtr. panchymag. Vngu. arthanit.

$$
\text { Q5 CICVR }
$$




\section{CVCVRBITA.}

589. CVCVRBITA lagenaria foliis fubangulatis to. mentofis bafi fubtus bighandulofis, pomis lignofis. Sp. pl. 1434 .

Cucurbita oblonga, thore albo, folio molli. Baub. pin. 333 .

Loc. America.

Anmua, fubcicur.

PHARM. CVCVRRITAE Semina.

QVar. fubdulcis.

Trita.

vis: edulcorans, refrigerans.

vsvs: Febres putridix, Agrypnia, Stranguria, Nepluritis.

comp. Semina IV frigida majora, Spec. diatragacanth.

590. CVCVBITA Citrullus foliis multipartitis. Hort. ups. 292.Sp.pl. 1435 .

Anguria Citrullus dicta. Banb. pin. 3 I2.

ıoc. Apulia, Calabria, Sicilia. Anma, Jubcicur. PHÁRM. CITRVLLI Semina.

eval. Cucurbitx.

Trita.

vis: Cucurbitx.

vsvs: Cucurbitz.

comp. Semina IV. frigida majora, Spec. diatragacanth. Pulv. haly.

\section{CVCVMIS.}

59I. CVCVMIS Colocynthis foliis multifidis, pomis globofis glabris. Hort upf. 293. Sp. pl. 1435.

Colocynthis, fructo rotundo, major. Baub. pin. $31 \hat{\jmath}$.

i.nc. Syria, Aleppo. Peremis, manfueta.

prarm. COLOCYNTHIDIS Pulpa, Semina, Refina, Extractum gr. 1 v.

Qval. amarilima, naufeofa. Rarior, beroica, catía. 
vis: draftica, emmenagoga, abortiva, anthelmin. thica.

vos: Obftipatio, Epilepfia.

comp. Enemata, Trosh. alhandal, Conf. hamech, Vng. arthanit. Pil. cochix, capitales, aurex, catholicx, gummat. Extr. panchymagog. cathol.

592. CVCVMIS Melo foliorum angulis rotundatis, pomis torulofis. Hort. cliff. 45r.ups. 292. Sp. pl. 1436 .

Melo vulgaris. Baub. pin. 3 ro.

Ioc. Calmucchia.

PHARM. MELONIS Semina, excorticata.

CVAL. fubdulcis.

vis: Cucurbitz.

vsis: Cucurbita.

comp. Sem. IV. frigida majora, Spec. diatragacanth.

593. CVCVMTS fativus foliorum angulis rectis, pomis oblongis fabris. Hort. cliff. 451. ups. 292. Sp. pl. I437.

Cucumis fativus vulgaris. Baub. fin. 3 Io.

LoC. Europæe culta.

Annus, cicur.

zharm. CV CVMERIS Semina, [Exuctus recens, Succus. ]

Rval. Cucurbitx.

vis: Cucurbitx.

Trita.

vovs: Cucurbitie. [Phthifis Sucs.]

comp. Sem. IV. Frigida majora, Spec. diatragacanth.

\section{BRYONIA.}

594. BRYONIA alba foliis palmatis utrinque callnfofabris. 17. Juec. 877. Sp. pl. 1438. Fl. dan.t.813. Bryonia alba, baccis nigris. Baub. pin. 297.

594. [Bryonin dinica Gach albre rubstimitur in regionibus, wai non hace, fed illat, crefcut an vires m.puales?] 


\section{MONOECIA. SYNGENESIA.}

Loc. Europa pagi. Peremis, infrequens. pharm. BRYONIAE Radix $习 j$. Extractum 3 j. Ficculce, Baccx, Semina.

eval. acris, amara, naufeofa. Ifetata, eximia. vis : purgans, pellens, anthelminthica, cmmenagoga. vsvs: Hydrops, Mania, Epileptia, Rheumatimus,

Ganglion, Sugillatio. Vngu. Pleuritis. comp. Catapl. refolvens. 


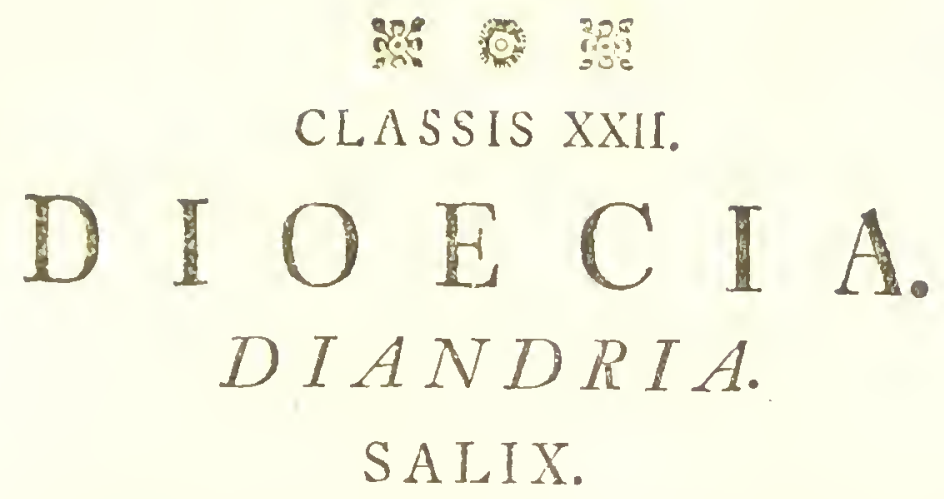

595 SALIX alba foliis lanceolatis acuminatis ferra. tis utrinque pubefcentibus: ferraturis infimis glandulofis. Fl. Juec. 903. Sp.pl. 1449.

Salix vulgaris alba arborefcens, Baub. pin. 473.

Loc. Europx urbes et pagi. Arlior, vulgaris. PHARM. SALICIS Folia, $\nabla$ (florm $)$

RVAL. amaricans.

IS : antivenerea.

Exoleta.

vsvs: Agrypnia, Dyfenteria.

comp. Balnea, Clyfinata.

\section{TETRANDRIA. V IS C V M.}

596. TYSCVMI album folis lanceolatis obtufis, cau. le dichotomo, fpicis axillaribus. F\%. Juec. 905. Sp. pl. 145 r.

Vifcum baccis altis. Baub. pin. 423.

LOC. Europx abores gummolix refinofieque. Fintex, parafiticus, infrequens. IHAR. VISCI [QVERNI] lignum, oo (bera. climmm).

Qvar. mucilaginora, graveolens. Rarior, preftans. VIS:

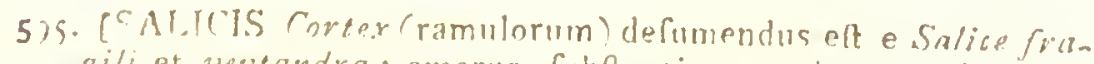
fili et pentandra: amarus, fubfypticus, tonicus, antifichrilis.] 5)6. [VISCI (2VFRCINI linnmm e Lorantho enropiro defumere jubet l'harm. wh/s. $\mu . ;$. ] 
vas: invifcans, lubricans.

rsvs: Epilepfia, Vertigo, Pleuritis, Dyfenteria. COMP.

\section{MYRICA.}

597. MYRICA Gale foliis lanceolatis fubferratis, caule fuffruticolo. Fi. free. 9i7. Sp. pl. I453. F\%. dan. $t .3^{2} 7$.

Rhus myrtifolia belgica. Baub. pin. 414.

1.oc. Europx borealis paludes. Frutex, vulgaris. PHARM. MYRTI BRABANTICAE Folia.

QVAL. amara, nidorofa, luteo-tinctoria, cerifera. Exoleta, proftans. vis: narcotica, phantanica, cephalalgica, exanthematica.

vsvs: Scabies! Pediculi.

EOMP.

\section{PENTANDRIA. PISTACIA.}

598. PISTACIA vera foliis impari-pinnatis: folio: lis Cubovatis recurvis. Sp.pl.1454. Piftacia peregrina, fructu racemofo f. Terebinthus indica. Baub. pin. 401.

loc. Perfia, Arabia, Syria; colitur in Italia, Si. cilia.

Arbor, inanfueta.

pharm. PISTACIAE Nuces, Nuclei mundati, (0). QVAL. dulcis, fubpinguis.

vis: nutriens, obtundens.

Rarior.

vsvs: Hectica.

Comp. Elect. diafatyr. Tabul. magnanimitatis. 


\section{DIOECIA. PENTANDRIA. 255}

599. PISTAClA Terebinthus foliis impari-pinnatis; foliolis ovato - lanceolatis. Hort. cliff. 456 . upp. 296. Sp. pl. I 455 .

Terebinthus vulgaris. Baub. pin. 400.

Loc. India, Africa, Aflyria, China, Cyprus, Ita. lia, Hifpania. Arbor, cicret. pHARM. TEREBINTHINA CYPRIA. Qval. Refina liquida, flava, fubdiaphana, amara, acris.

Rarior, preflans.

vis: refolvens, emolliens, vulneraria, diuretica urina violacea, eccoprotica.

vsvs: Phthifis, Tuflis, Vlcus, Gonorrha'a.

comp. Andromach. Mithridat. Balfam. fulphur. terebinthinatus $G$ t. $\mathrm{X} ; \Omega$ anhaltinus.

600. PISTACIA I entifczs foliis abrupte pinnatis: foliolis lanceolatis. Hort. cliff. 456. upf. 296 . Sp.pl.145t. *. Lentifcus vulgaris, Baub. pin.399.

Loc. Sicilia, Italia, Lufitania. Arbor, manfueta PHARM. LENTISCL Lignum.

R. Lentifcus ex Chio. Toumef, itin. 2. p.66. LOC. Chios.
Arbor, peregrima. pharir. MASTICHES Refina alba, granulata, Sj: rupus, (O), 0 .

QVAl. Lign. ficca, Refina fragilis, pallida, aromatica.

Vhitata, preftans. vis: corroborans, flomachica, vulneraria. vsvs: Tufis, Catarrhus, Odontalgia, Arthritis, Calculus, Stomacace.

comp. Pil. fine quibus, Pil. ammoniacx, Pil. catholicx, Hiera fimplex, Troch. carabe, Troch. hedycroi, Vngl. martiatum, Empl. Alomachal. Empl. oxycroc. Empl. diaphoret. Empl. opodeltoch, Empl. nervinum, Empl. gummat. Empl. *alaminar. Empl. fracturis, Cerat. Betonic. 


\section{SPINACIA.}

6or. SPINACIA oleracea frucibus leflilibus. Hort. clift. 457.upl. 296. Sp. pl. 1456.

Lafatinum hortente f. Spinacia femina fpinofo. Baub. pin. II 4 .

Loc. hodie Horti noftri. Ammu, ci is PHARm. SPINAClAE Herba, Semina.

QVAl. aquofa, oleracea, inlipi'a. Lixileta, fuperfira. vis: diluens, humedars, eccuprotica.

vsvs: cibarius.

COMP.

\section{A N NABIS.}

602. CANNABIS fativa. Hort. ulfJ. 457. upp.297. Sp. pl. 14.57 .

Cannavis lativa. Buhb. Min. 320.

Loc. Japonia, India orientalis.

PHARm. CAVithis Semina, (1).

CYal. virofa Serm. olenta. Rerior, preftnns. vis: rarcotica, phantaflica, dementans, anodyna, repellens.

vsrs: Gonorrhcea, Leucorrhœa, Icterus.

COMP.

\section{HVMVI, VS.}

603. HVMVI,VS Lipulus, Fi. fuec.908. Sp. pl. 1457. Lupulus mas (femina rectius). Baub. pin. 298.

loc. Europa lepes.

Peremis, cicur. PHARM. LVPVII coni.

QVAL. anara, nidorola. Rnrior. vis: tonica, fubnarcotica, phantaftica, anodyna. vsvs: Nephritica, Phrenitis, Luxatura, Alopecia.

Sem. Obftipatio.

COMP. 


\section{HEXANDRIA. SMILAX.}

604. SMIILAX Sarfaparilla caule aculeato angulato, foliis inermibus ovatis retulo - inucronat's trinerviis. Sp. pl. 1459 .

Smilax, viticulis alperis, virginiana, folio hederaceo lævi, zarza nobilifima. Pluk. alm.348.t inr. f.z. Loc. Virginia, Mexico., Perennis, manfueta. I'HARM. SARSAE PARILLAE Radix. QYAL. ficca, amaricans, glutinola. Trite, preftans. vis: diuretica, fudorifera, purificans. vsvs: Syphilis, Arthritis, Cachexia. comp. Decotta lignorum.

605. SMILAX Cbina caule aculeato teretiufculo, foliis inermibus ovato - cordatis quinquenerviis. Sp pl.1459. [Gmel. it. 3. p. 32. t.6.]

Smilax minus finola, fructu rubicundo, radice virtuofa. Kempf. anien. 781. t. 782.

zoc. Perfia, China, Japonia. Perennis, peregrina. PHARI. CHINAE Radix.

QVAL. ficca, inodora.

vis: purilicans.

vsvs: Syphilis, Podagra.

comp. Decocta lignorum.

\section{OCTANDRIA. POPVLVS.}

606. POPVI.VS nigra foliis deltoidibus acuminatis ferratis. Hil. Juec. gro. Sp. pl.1464. [Pall. rolj. r. p. 66.] 
Populus nigra. Bantb. pin. 429.

LOC. Europa aufralior. PHARM. POPVLI oculi (gemma rectius). Cyal. refinola.

vis: obtundens, emolliens, foporifera.

Arbor, cicur.

vsvs: Diarrha, Dy fenteria! Combunura, Arthritis. cons. Vngu. populeum.

607. POPVLVS bnlfamifera foliis ovatis ferratis fubtus albidis, ftipulis refinolis. Sp. pl.1464. [Pall. rolf. I. p.67. t.41.]?

Tecomahaca. Hernand. mex. 55. Pluk, alm. 360. t. 28 I. f. 2.

roc. Mexico, Madagafiar. Arbor, manfueta. pHaRm. TACHAMiAHACA Refina.

QVAL. arida, friabilis, variegata, fubdiaphana, ambrofiaca.

VJitata, prestans.

vis: leniens, maturans.

vsus: Dolores, Odontalgia, Gaftrica, Cephalalgia,

Coplofis, Vomitus, Hyfteria.

comp. Fimpl. opodeltoch, Empl. matricale, Empl. ftomachale.

\section{RHODIOLA.}

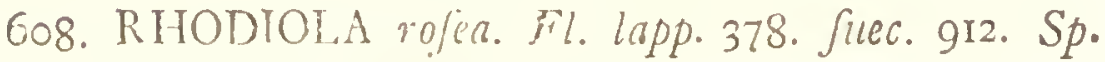
pl. 1465. Fl. dim t. 183 .

Rhodia radix. Batb. fin 286.

Loc. Alpes Lapponica aliaque. Peremis, cicur. PHARM. RHODIAE Radix.

Qral. rubicunda, ftyptica, fragrans, rofé odore.

Exoleta, cximia.

Vis:

6c6. [POPVLI oculos e Populo balfrmifrra colligendos elle monet ling mus wad.502.]

co7. Rel TACAMAHACAMI 7rewius negat elle Pojulum balfawifiram; Gacquinus afferit elle liagurum oftandram. 
vis: adlringens.

vsrs: Hernia, Leucorrhœa, Cephalalgia! Hyfteria, Dyfenteria.

COMP.

\section{ENNEANDRIA. MER CVRIALIS.}

6og. MERCVRIALIS anmua caule brachiato, foliis glabris, Horibus ipicatis. Hort. cliff. 46r. ups. 298. Sp. pl. 1465 .

Mercurialis tefliculata feu mas. Baub. pin. I2I.

Loc. Italia, Anglia, Gallia. Amma, cicur. PHARM. MERCVRIALIS Herba, Mel.

QVal. fufpecta. Trita, externa, dubir. vis: hypnotica, purgans, repellens, cofmetica, non emolliens.

vsus: Syphulis.

comp. Enemata, Spec. pro clyferibus, Syrup. prophylacticus.

\section{DODECANDRIA. MEN ISPERM VM.}

6ro. MENISPERMVM Cóccuizis foliis cordatis retufis mucronatis, caule laccro. Sp.pl 1468.

Natiatam. Rbeed. mal. 7. t. I. f. I. Pluk. phyt. 345. f.2. Rumiph. amb.5. t. 22 .

roc. Malabaria. Peregrina, fera. PHARM. COCCVLI INDICI Fructus.

QVAL. reniformis.

vis: venenata, phthiriaca.

vsvs: pediculis, rilicibus.

COMP.

Externa. 


\section{MONADELPHIA. JVNIPERVS.}

6ri. JVNIPERVS Sabina foliis oppofitis eredis de. currentibus: oppofitionibus pyxidatis. $S p \cdot p l .1472$. Sabina folio cuprefli. Banls. pin 487.

Loc. Sibiria, Tataria, Olympus. Ambrenta, cicur. pharm. SaBINAE Herba, lifjentia, $\nabla, 0^{\circ}$

Qval. amaricans, hircola. Trita, preflans. $\mathbf{v}$ Is : emmenagoga! abortiva, anthelninthica, exanthematica, diuretica, depilans.

vsus: Odontaluia, Tinea, Gangrena, Scabies. comp. [Hippiatrorum pulveres.]

612. JVNIPERVS commmis fohis ternis patcntibus mucrnnatis bacca longioribus. Fl. fuec. 915. Sp. 1)l. 1470 .

Juniperus vulgaris fruticola. Banth. pin. 488.

Loc. Europa fylva. Arluefcula, vuligatifima. pharm. JVNIPERl Lignum, 0 o, Cineres, Baccx, Rol, $\nabla, \Omega, \circ$ o.

SANDARACA Relina.

eyal. ficca, aromatica, refinofa. Trita, preftans. vis: pellens, carminativa, diuretica urina violacea. calcfaciens, alexiteria, mundificans.

vsrs: Cophofis, Calculus, Afthma, Catarrhus, COMP.

613. JVNIPERVS lycia foliis ternis undique imbrica.. lis ovatis obtufis. Roy. lugdb. go. Sp. fl. rafr.

Cectrus, folio cuprefi, major, fruclu Havelcente. Baub. pin. 487 .

Loc. Alrica, Aethiopia, Arabia. Arbor, peregrina. Vermes 0 .

PHARM.

613. [OLIBANVM ex hac arbore defumtum effe alii fiatunt, alii asegant; et Sumbracam non prioris fed lrujus efle re. finam 


\section{DIOECIA. MONADELPHIA. 261}

PHAR.M. OLIBANI (THVRIS) Refina, granulata, $0^{\circ}$ CrAl. refina llavefcens, pellucida, amaricans. Rarior. vis: vulneraria.

vsis: Pleuritis epidenica, Ophthalmia, Catarrhus. CONP.

\section{CISS A MPELOS.}

6r4. CISSAMPLLOS Pareira foliis peltatis cordatis emarginatis. Sp.pl. 1473 .

Clematis baccifera glabra et villora, rotundo et umbilicato folio. Plum. amer. 78 t. 93 .

Loc. America meridionalis. Perennis, peregrina. PHARM. PAREIRAE BRAVAE Radix.

QV.t. dulcamara. Rarior, eximia. Vis: diuretica, demulcens, purificans.

vsvs: Calculus! Dyfuria! Arthritis, Podagra, Hy。 drops, Gonorrhœa, Ifterus.

comp. Decoct. lignorum.

\section{SINGENESIA. R VSCVS.}

6r5. RVSCVS aculeatus foliis fupra floriferis nudis. Hort. cliff. 565. upf. 300. Sp. pl.1474.

Rufcus Bauls. pin. $47^{\circ}$.

Loc. Italia, Gällia.

pharm. RVSCI Radix, Semina.

erat. fubciulcis, dein amara.

Rarior.

vis: combobrans, emmenagoga, diuretica, corraria. vsvs: Hydrops! Aleites, ICterus.

Comp. Rad.V. aperientes majores; Benedicta laxativa.

$$
\mathrm{R}_{3} 616 \text {. }
$$

finam volunt alii; lis pendet [F gunip. thurifera provenire, in rhurm. Fuec. afferitur.]

6in. PADVTA rafefopns foliis ovatis fibtus villofis. Aubl. guian. Grs. Ixis. 250. Pareir brave noinine in Guiana venis es pio ca in Europan invehitur, tche Aublctio,] 
6r6 RVSCVS Hypogloflum foliis fubtus floriferis fub foliolo. Hort. cliff 466. up/ 300. Sp.pl. 1474.

Laun alexandrina, fructu pediculo infidente. Baub. pin. 304 .

coc. Hungaria, liguria. Suffrutex, manfuetus. PHARM. VVVLARIAE Herba.

Qval. Ayptica, infipida, inodora. Exoletr: vis: adfringens.

vsvs: Mrolapifus uvulx.

COMP, 


\section{สลत \\ CLASSIS XXIII.}

\section{P OL Y G A M I A. MONOECIA. VERATRVM.}

617. TER ITRVM allum racemo fupradecompofito, c)roilis erectis. Sp. pl. 1479. Facq. auftr. 4. t.335. Helleborus albus Hore lubviridi. Banh. pin. 136.

Loc. Rulia, Sibiria, Auftria, Helvetia, Italia, Græcia in montolis. Peremis, cicur. PHARM. HELLEBORI ALBI Radix Gr. vij. a Al. arris, nauleola. vis: draffica, emetica, fernutatoria!

rivs: Cophofis, Mania, Epilepfia, Quartana, Scabies, Phthiriafis,

cosp.

618. [VERATRYM Sabadilla racemo fimplici? corollis patentibus. Retz. obf. I. p. 29.

Vclamm, quod mexicanum Sabadilli femen. Vanderbech act. n. cur. I. app. 120. Berg. mat.513.] Los. Níexico.

ptiarir. SABADILLI Scmina.

Q Q Y A. acris, amara, cauffica.

vis: draftica, volnitoria, phthiriaca, toxica.

rsis: Pediculi.

conp.

\section{$\triangle N D R O P O G O N$}

6rg. ANDROPOGON Scbrnantbus panicula fpiculis conjugatis ovato-oblongis, rachi pubefeente, flo. fulis deflilibus: arita tortuola. Sp.pl.r48r.

R 3 Jun.

617. HELLFEBORI ALSBI rart. a Veratro nigro quoque defuml

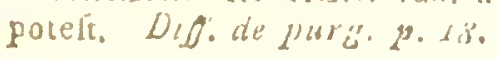


Juncus odoratus aromaticus. Baub. pin. Ir. Rumph. amb. 5. p.181. t. 72. f. 2.

LOC. Arabia.

PHARM. SCIHOENANTII Herba.

QYAL. fragrans, aromatica.

Exoleta.

vis: emmenayoga, carminativa.

vsvs: Vomitus, Vleera veficx.

comp Andromach. Mithridat.

620 A $:$ DROP'OGON Navius paniculx racemis fupradecompofitis proliferis. F\%. a.eyl.45 Sp pl. I482. Calamus odoratus matthioli. Barib. pm i 7 .

Loc. Zeyloria. Peremis, peregrina, dubia. PHARM. SPICAE (INDICAE) NARDI Radix.

QYAl. fragrans. Eximin, imufitata. vis: alexiteria, cordialis, ftomachica, anthelminthica. vsis: Mebilitas, Hrmorrhagia.

romp f tiarrhodon, Andrromach. Mithridat. PhiIonium. Benedicta, Hiers picra, Thochil. hedy. croi, Vigu. martiatum, Ving nardinum.

\section{PARIETARIA.}

621. PARIETARIA offurinalis foliis lanceolato-ovatis, pelnuculis dichotomis, calycibus diphyliis. Hort. cinfl. 469. Sp.pl. 1492. Fl dhan. t.521.

Parietaria officinarum et diofconidis. Baub. pin. I2I. Loc. Europa aufralioris inuri, ruderata.

PHARM. PARIETARIAE Herba.

Perennis, cisur.

Qrat. aquofo-infipida.

vis: emolliens, diuretica, obtundens.

vsve: Hydrops, Calculus, Tabes, 'Tuflis, Stranguria.

comp. Herbe $\mathrm{V}$ emollientes, Syr. althax, Enemata. 


\section{A TRIPLEX.}

622. ATRIPLEX bortentis caule erecto herbaceo, foliis trangularibus. Hort. cliff.46g. up. 303. sp. pl. 1493 .

Atiplex hortenlis rubra. Bauls. pin. IIg.

Lo... Sibiria

shmina, cicur. sharm. ATRIPLICIS SATIVAE Herba, Sumina. QYAl. aquolia, oleracea, infipida.

Exwleta, infida, fuperfua.

vrs: diluens, humectans, refrigerans. Sem. purgans, emetica

vsis: Inthaminatio. Sem. Rhachitis.

coins. 考 jutteta.

\section{TER MINALIA.}

623. TFRMINALIA Benzwin foliis lanceolatis. Suppl. pl. 4i4. of icq bort. 3. p. 51. t. 100.?

Croton Benwoë foliis linearibus fubrepandis nudis:

venis rubris, Mant.pl.2 p. 297.

Loc Sumatra, Jara, Siam. Arbor, peregrina. PHARM. MitidoES Refina, Timetura, Fiores.

Qut. refinola, graveolens, fragilis. Trita, preftans. vis: nervina, expectorans, bailianica.

vsvs: Rubedo facici.

comp. Eimpl. de Storace.

\section{OPHIOXYLON.}

624. OPIHOXYi.On jerpentimum. Sp.pl. r478.

Ligufrum folis ad fingula intemodia icriais. Burm. zeyl. I41. t. 64 .

\section{R 5}

LOC.

623. Trunci lignum, ex Benzo: oficinarum, qund fecum atuporravit D!). Ihunberg, cortice lingulari valde reled corti-

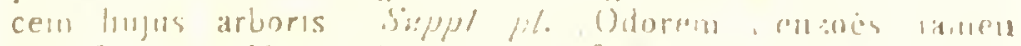

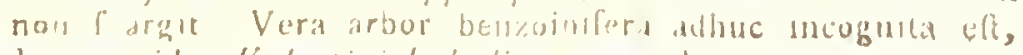
de qua vid. Valonitini lunt. lit. 8.457 . J 
u oc. Zeylona. Arbor, peregrina. YHARM. SERPENTINVM Lignum $5 / 3$.

Q: Al. amara.

vis: pellens, fudorifera.

vsvs: Quartana! Morlura.

C()MP.

Parca.

\section{MIMOSA.}

625. MM!OSA nilotica frinis Ripularibus patentibus, fuliis bipinnatis: partialibus extmis glandula interfinctis, fpicis globofis pedunculatis. Sp. pl. 1506. Hi:\|elq. it. 475 .

Acacia vera. Baub. bjjt. I. p. 429. Vesl. egypt.t.8. Plak. alm. 3. t.123. $f$. 1 .

ıoc. Arabia petra, Acgyptus. Arbor, ferc. PHARM. ACACIAE VERAE Succus. ARABICVM Gummi.

QVal. Succ. ftyptica. Gum. mucilaginofa, flavefcens, infipida.

V fitata, proftans. vis: Succ. adfringens. Gum. invifcans, lubricans, obtundens, nutriens, maturans.

vsvs: Succ. Hxmoptyfis, Menorrhagia, Prolapfus; Gum. Sphthalmia.

conp. Sucte. Andromach. Gum. Mithridat. Troch. carabe, Lob. Fanum.

626. MMOSA Senegal finis ternis: intermedia reflexa, foliis bipinnatis, floribus fpicatis. Sp. pl. I506. Acacia. Bath. pin. 392. Alp. cegypt. 9. t. 15. Pluk. phyt. 25I. f. I.

zoc. Arabia.

PHARM. SEREGAL Gummi.

Arbor, fera.

NAL. pracutuntis, praflantior. Vfitata, preftans. 


\section{POI.YGAMIA. DIOECIA.}

vis: pracelentis.

vsis: pricedentis.

COMP.

627. IIMOSA Catechu fpinis Aipularibus, foliis bipinnatis multijugis: glandulis partialium folitariis, fpicis axillaribus geminis ternisve peduncularis. Stuphl. pl. 439.

Coira vel Kaira. Lerr med. obs. and inquir.5. p.51. tah 4 .

LOC. India orientalis.

Arhor, peregrina. PHARM CATECHV (Succi Japonici) sij, Tinciura. Qrat. gummolo-refinofa, obleure rufia, inodora, fubamara poftea dulcior, ftyptica. Trita, eximia. vis: adftringens.

vsvs: Prolluvia, Diarthoca, Coryza, Stomacace. cosip. Trocb. catechu cum molcho, fine nofcho.

\section{$D I O E C I A$. FRAXIN VS.}

628. FRAYINVS excelfror foliis forratis, floribus apetalis. Fl. Juec. 926. Sp. pl. 1509 .

Fraxinus exceifior. Baub. pin.4r6.

uoc. Europe prata.

Arbor, vulgaris. PHARm. FRAXM Lignum, $\theta$, Cortex, Folia, Semina (lingua auis).

Cy.A. Cort amara; Sem aromatica, fingularis. Vfltata.

vis:

627 [Terran Gaponicam D. Fothergill, qui D. Kerr requitur, e Minola fpicaca Pluk. mont. 8. 2.320.f. 5. 6. prove-

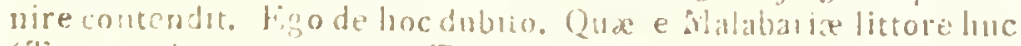
(Trangmbaluan veuse, Tamulis iblagalom dicinr. Vora Terra Japonica Tamulis Ki心balli audit, et a pauperioribus

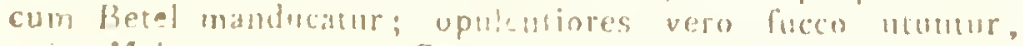

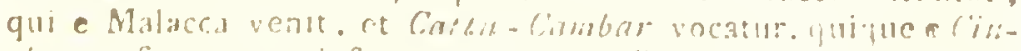

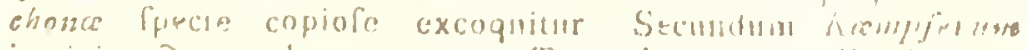
in ilise 2 inn hicc vera crit Terra Japonica. Limbo ap. Letz. $f a f c \cdot 4 \cdot \mu \cdot 5$. 
VIs: adfringens; Sem. ficcans; Sal. diuretica. vsis: Hamorrhagia, "Tertiana. [Fol. Morfura Der. pent.] Sem. Obefitas.

comp. El. dialatyr.

629. FRAXMVS Ormus foliis ferratis, floribus co. rollatis. Moot. cliff. 470. upp.304. Sp.pl 1510.

Fraxinus Jumilior f. aitera theophrant. Baub.pin. 416. I.oc. Calabria, Sicilia. Arbor, mmnfuta. PHARM. MIXNA CAIABRINA ऊ/3. granulata, tabulata, Morjule.

Qvar. mellea, granulata, rubicunda, fubdiaphana, dulcis.

Trita, eximia.

vis: edulcorans, eccoprotica! obtundens, thoracica. vsvs: inhma humorale, Colica nephritica. comp. Laxans debilium.

\section{IOSPYROS.}

630. DIOSPYROS Ekemus glaberrima, foliis oblongis utrinque glaberrimis coriaceis venofo-reticulatis, lloribus axillaribus folitaris fellilibus. Supte. pl 440 .

coc. Indix orientalis fylva vafte. Arbor, peregrina. PHARM. EBENI Lignum.

QVAL. in aqua fubmergitur.

Exoletc.

VIS:

629. [MANNAM CALABRINAM Fraxinus rotudifolia Mill.

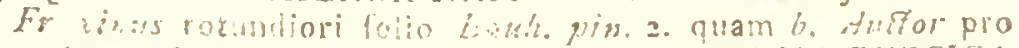
variate Crm tabuife videtm, exfudat. MANNA PRRSICA

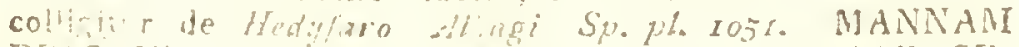

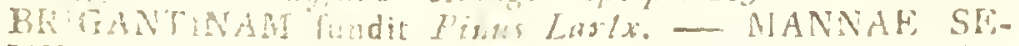

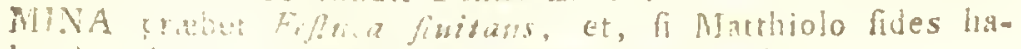

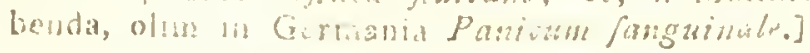

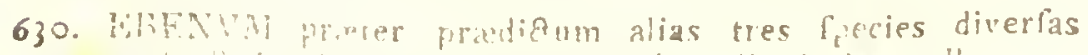
pron ir liate pasian jo. (Wariat colore ligni nigro, albo, va-

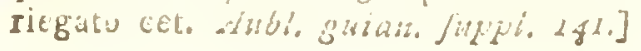


Vis:

vsvs: meclanicus.

cosp. Decuciun lignorun.

\section{$P A N A X$}

631. P.NAX quinqquefoizm foliis ternis quinatis. $S p$. pl. 1512 .

Aureliana canadenfis, Sinenfibus Ginfeng. Lufit.

ging. r. Trewo. ebret.t.6. f. I.

Loc. Canada, Tataria, inter gr. $39-4.7$.

Perenmis, manfueta.

PHARM. GINSENG Radix Bj.

Q bAl. aromatica.

ris: cordialis, analepica.

Rarior, pretiofa.

vsvs: Atecnia, Debilitas, Marafmus, Senectus.

COMP.

\section{$T R I O E C I A$. CERATONIA.}

632. CER ATONIA Siliqua. Hort. ups. 296. Sp. pl. 1513.

Siliqua edulis. Baub. pin. 400.

r.oc. Ludæa, Syria, Apulia, Sicilia. Arbor, manfleta. PHAR.M. DVLCIS SULIQVA.

Qvat. dulcis, ficca.

vis: edulcorans, expectorans.

Rarior, preftans.

vsus: Soda, Raucedo, Afhma, Tuffis, Ophthalmia.

comp. Syr. diacodii cum filiquis. Decoit. infantum.

\section{FIC VS.}

633. FICVS Carica foliis palmatis. Hort. cliff. 47. 2 ip $\int .305 . S p \cdot p l .2513$.

Ficus communis, Baulb. pin. 457. 
coc. Grecia, Hifpania, Italia. Arbor, manfueta: PHARM. CARICAE pinguls.

QVAl. dulcis, mucilaunofa, ladefecns. Trit ,eximia. $\mathbf{v}$ s : nutriens, impinguans, obundens. lubricans, emolliens, maturans, hepatica! difuretca, eccoprotica, exanthematica.

vsve: Tullis, Colica laturnina, Exanthemata, Bubo, Anthrax, Dyffocia, Obftipatio; nocet Scabiofis et gingivis ulcerofis.

comp. Enemata. 


\section{CLASSIS XXIV.}

\section{CRYPTOGAMIA. FILICES. EQVJSETVM.}

634. FQVISETVM arvense fcapo fructificante nudo, farili frondofo. Fl. fuec. yzs. Sp. pl. 1510 . Enuiletum arvenfe longoribus fetis. Baub. pin. I6. roc. Europer prata agrique. Ferems, frequens. PHAR.r. EQVISE'TI Lluba.

य $\mathrm{AL}$. ficca.

vis: alltringens! diurelica!

Exoleta, eximia.

rsys: Hamaturia! Gonorthora benigna, Prolluvia, Whithifs.

cons.

\section{CYCAS.}

635. CYCAS circinalis frondibus pinnatis: foliolis linearibus planis. Sp. pl. i658. Mant.pl.2.p.305. Tolla-panma. Rbeed. mal.3.p. 9. t.13-21. sor. Siarbaria, Ambrina, Japonia. Arbor, fera.

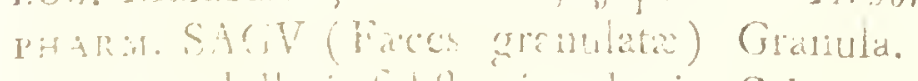

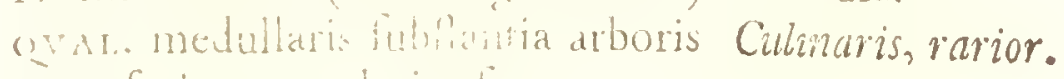
vi: farinaceo-glutinola.

Ysus: nutriens.

conp. Cibus.

ASPLE.

684. Tituifetum hiemale in Suecia officinale ent Equijeti majoris, ficut arvenfe minoris numine. Vires exdem. I3erg. mat.5-5.]

635. [Cranula Sagu non dat Cycas circinalis, ut vulgo credi-

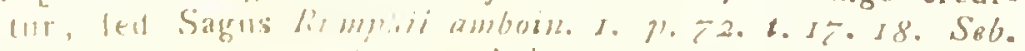

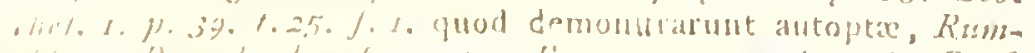

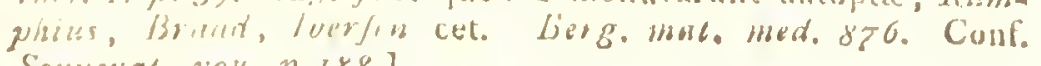
Sonuerab. voy. p.188.] 


\section{ASPLENIVM.}

636. ASPLENIVM Scoipiendrizm frondibus fimplicibus cordato-lingulatis integerrmis, nipitibus hirfutis. Fi.juec. 941. Sp. pl. 1537.

Lingua cervina officinarum, Baub. pin. 353

coc. Germania, Gallia, Italia. Peremis, cicur. PHARM. SCOLOPENDRII Herba.

Q RAL. ficca.

Rarior.

vis: adfringens.

vsve: Hamoptyfis, Hyfteria, Palpitatio, Rachitis,

Cachexia.

COMP.

637. ASPLENIVM Ceterach findibus pinnatifidis: lobis alternis conlluentibus obtufis. Hort. clifl. 474 . Sp.pl. 1538.

Ceterach officinarum. Buth. pin. 354.

zoc. Italia, Wallia, Germania. Peremis, manfueta. PHARM. CETERACH [ASHLENII] Herba.

QYAL. ficca.

vis: tonica, fplenica.

vsvs: Hydrops, Aflima, Obftructiones vilcerum. COMP.

638. ASPLENIVM Trichommes frondibus pinnatis: pimis fubrotundis crenatis. Fl. /2tec. 942. $3 \% . \mathrm{pl}$. 1540. Fl. dun. $t$ ing

Trichomanes f. Poiytrichum officinacum. Buh. pin. $350^{\circ}$.

LOC. Furopæ ruves.

PHARM. TRICHCMANIS Herba.

QVAL. ficca.

Peremm, oulgaris.

vis: fubadftringens, pectoralis.

Rarior.

VSVS:

CUMP. 
639. ASPLENIVM Ruta muraria frondibus alternatim decompolitis: foliolis cuneiformibus crenulatis Fl.fiec. 943. Sp. pl. 1541. Fl. dan. t. Igo.

Ruta muraria Baub. pin. 356.

Loc. Eurnpa.

PHAR.Y. RVTAE MVRARIAE Herba.

Perennis, infrequens. QVit. ficca.

vis: tonica.

vsvs: Cachexia, Scorbutus, Rhachitis.

COMP.

\section{POL Y PODIVM.}

640. POLYPODIVM vulgare frondibus pinnatifidis: pinnis oblongis fubferratis obtufis, radice fquamata. Fl. fieec. 944. Sp. pl. 1544.

Polypodium vulgare. Baub. pin. 359 .

LoC. Europæ rupes.

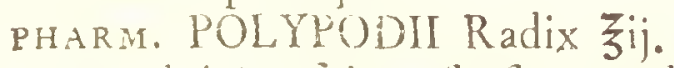

Perennis, frequens. QVAL. dulcis, fubnauleofa, tandem amara.

Tritn, preftans.

vis: edulcorans, incraflans, expectorans, eccopro. tica.

vsvs: Colica, Tuflis, Arthritis, Rachitis!

comp. Decocta compofita.

64I. POLYPODIVM Filix mas frondibus bipinnatis : pinnis obtufis crenulatis, nipite paleaceo. Fl. fuec. 946. Sp.pl. 155r.

Filix non ramofa dentata. Baub. pin. 358 .

Loc. Europx fylve.

PHARM. FILICIS Radix.

Perennis, vulgaris,

QVAL. amarulenta.

VIS: anthelminthica! tonica.

Vistata. 


\section{4}

\section{CRYPTOGAMIA. MVSCI.}

vsvs: Scorbutus, Rachitis, Melancholia? Txuia? Podagra.

comp. Decocta.

\section{ADIA NTVM.}

642. ADIANTVM Capillus veneris frondibus decom. pofitis, foliolis alternis: pinnis cunciformibus lobatis pedicellatis. Sp. pl. 1558. Hudf. angl. 391. Adiantum foliis coriandri. Baub. pin.'355. Loc. Italia, Gallia.
Perenmis, manfueta. pharm. CAPILLI VENERIS Herba, Syrupris. Qval. ficca.

vis: corroborans, fplenitica, diuretica. Rarior. vsvs: Obftructio vifcerum.

comp. Syr. althax, Lob. pulm. vulp.

\section{$M V S C I$. LYCOPODIVM.}

643. LYCOPODIVM clavatum foliis fparfis filamentofis, fpicis teretibus pedunculatis geminis. $\mathrm{F}$. fuec. 956. Sp. pl. 1564 . Fl. dan. t. 126. Mufcus terreftris clavatus. Baub. pin. 360 .

上ос. Europx fylvx acerofx. Peremnis, vulgaris. PHARM. MVSCI CLAVATI Herba, Farina (pollen).

CVAL.

Exoleta.

vis : exficcans.

vsvs: Dyftocia? Plica, Ecclampfia. Poll. Excoriatura.

cOMP.

644. L. Y.

642. [Adiantum pedatum commode huic fubfituitur in America reptentrionali et Gallia; ficut in promontorio bonae fpei Adiantum cithiopicum Thant. wed. afr. 4.] 
644. LYCOPODIVM Selago foliis fparfis octofariis, caule dichotomo erecto falligiato, Horibus fparfis. Fl. fuec.955. Si. pl.1565. Fl dan. $t$ 104.

Mufcus erectus ramofus faturate viridis. Banb. pin. 360. Dill. mufc. 435. t.56.f. $\mathbf{x}$.

roc. Europre junipereta.

YHARM. MVSCI ERECTI Herba.

QVAL. virulenta.

Perennis, vulgaris.

vis: draftica, abortiva, phthiriaca.

Exoleta, beroica. vsvs: Hydrops, Pediculi.

COMP.

\section{P OLYTR I C IV M.}

645. POLYTRICHVM commune caule fimplici, anthera parallelepipeda. Fl. fuec. 966. Sp. pl. 1573. Polytrichum aureum medium. Banb. pin. 356. Dill. $m i c c .424 \cdot t \cdot 54 \cdot f \cdot 2$.

Ioc. Europx arida.

PHARM. ADIANTI AVREI Herba.

QVAl. cruda.

Exoleta, imutilis.

VIS:

rsvs: Pleuritis?

COMP.

\section{A $L A E$. LICHEN.}

646. LICHEN faxatilis imbricatus, foliolis finuatis fabris lacunofis, fcutellis badiis. Fl.fuec. ror s. Sp.pl.160g. Lichenoides vulgatiflimum cinerco-glaucum Lacunolum et cirrhofum. Dill. minfc. 188. t. 24. $\int .83$. 1.Oc. in arborum truncis; Ciclectus? in Cranio humano. PHARM. VSNEAE Herba.

Parafinica. QVAL.

vis: fuperftitiofa, Exoleta, inutilis. 


\section{CRYPTOGAMIA. ALGAE.}

vsvs: Epilepfia? Hamorrhagia?

comp. Vngu. armarium.

647. LICHEN islanaiicus foliaceus adfcendens laciniatus: marginibus elevatis ciliatis. Fl. Juec. 1085. Sp. pl. 161. Fl. dan. t.155.

Mufcus islandicus purgans. Barth. alt. 1674. p. 125. zoc. Luropx fylya fterilifimx.

PHARM. MVSCI ISLANIDICI Herba.

CYAL. farinacea, amaricans.

Lerreflits.

vis: eccoprotica, nutriens, pectoralis.

Rarior.

vsvs: Phthilis, Tullis, Hamoptylis.

COMP.

648. LICHEN pulmonarius foliaceus laciniatus obtufus glaber: fupra lacunofus, fubtus tomentofus. Fi. Juer. 1037. Sp. pl. 1612.

Lichenoides pulmonaiium reticulatum vulgare, marginibus peltiferis. Dill. mulsc.212.t. 19. $f$. 113 .

zoc. Quercus Fagique antiqux. Parafitica. PHARM. PVILMONARIAE ARBOREAE Herba. QVAL. cruda. Litita, preflans.

vis: thoracica.

vsvis: Icterus, Tufis.

COMP.

649. LICHEN apbtbofus coriaceus repens lobatus ob. tufus planus, verrucis fparfis, pelta marginali adfcendente. Fl. fuec.1098. Sp. pl.1616. Fl. dan. t.767. f. 1 .

Lichenoides fubglaucum cumatile, foliis tenuibus eleganter laciniatis. Dill. musc.197. t. 26. f.99.

Loc. Europa junipereta.

Vulgaris.

PHARM.

649. Hic mufcus nobis non officinalis, fed rufticis noftratibus ulitatifimus. 
FHARM. MVSCI CVMATILIS Herba.

Cral. tetra, mephitica.

Imufitata.

ris: draftica.

vsis: Aphtha, Vermes.

COMP.

650. LICHEN caninus coriaceus repens lobatus obtules planus: fubtus venofus villofus, pelta mar. ginali adfcendente. Fl. Suec. 1100. Sp. 1). 1616. Fi. dum.t. 767 . f.2.

Lichenoides digitatum cinereum, lactuca foliis finnuolis. Dill.mufc.200. t. 27. f. 102.

Lor. fylve acerolx lapidesque

Vulgaris. pharit. MVSCI CANINI Herba.

CYAL. tetra, mephitica.

vis: mundificans, pellens.

Inufitata.

vsis: Hydrophribia! Rabies!

conp. Pulv. antilyflus Öjv.

651. LICHEN cocciferus fcyphifer fimplex integerrimus, fipite cylindrico, tuberculis coccineis. Fl. /uec. I109. Sp). $\mu$. 16r8.

Lichen pyxidatus, oris coccineis et tumentibus. Vail. parif. 15. t. 21. f. 4 .

I.oc. Furopre fylva.

PHARM. MIVSCI YYXIDATI Herba.

Vulgaris.

RAL. ficca.

Rarior.

vis:

vsve: 'Tufis convulfiva,

coirle.

652. I.ICHEN Roccella firuticulofus folidus aphyllus fubramolus, tuberculis alternis. Sp. pl. 1622.

Coralloides conniculatum fafciculare tinctorium, fuci terctis facie. Dill. mujc. 120. t.1\% J.39.

$$
\text { S } 3
$$

L.OC.

650. Nondum officinas noftras intravit. 
soc. Archipelagi, Canariarum fcopuli marini.

FHARM. ROCCELLAE Herba.

Peregrina.

Qval. fublalla, fubacris, tinctoria rubra. Trita. VIS:

vsis: tinctorius vini.

COMP.

653. LICHEN plicatus filamentofus pendulus: ramis implexis, fcutellis radiatis. Fl. fuec mz2. Sp. fil 5623 .

Vsnea vulgaris, loris longis implexis. Dill. mufa. 56. $t$ II. $f$. I.

Loc. Europe fylva antiqux. Parafiticus. PHaRM. MVSCI ARBOREI (QVERCINI) Herba. CYAL. ficca.

Exuleta.

vis: adfriingens.

rsvs: Excoriatura, Hæmorrhagia, Hernia umbi. licalis.

COMP.

\section{$F \nu N G$ BOLETVS.}

654. BOLETVS Larisinus [conicus pulvinatus lavis inxqualis, tubulis fubtilifimis. Ger. gallopr. I3. Jucq. mife. I. p 172. t.lb 20. 21. defcr.et ic. Wubferit. Pall. roff.r. p. 3.]

Agaricus f. Fungus laticis. Baub. pin. 375.

roc. Alpium Tridentiarum, Tyrolis, Helvetix; Delphinatus, [Ruffia, Sibirix] Larices. Peregrinus. PHARM. AGARICI OPTIMI ctum) $\ni i j$. 
ayd. fubdulcis, mox amara, acris, naufeofa.

Trita, eximia.

vis: fubadnringens, eccoprotica! flatulenta, calefaciens, anthelminthica.

vsis: Tullis.

comp. Pil. agarici, Trocb agarici $\boldsymbol{z}^{\mathrm{ij}}$, Hiera picra, Extr. cathol. Extr. panchymagog. Andromach. Mithridat. Conf. hamech. (non pulveres).

\section{PEZIZA.}

655. PEZIZA Auricula concava rugofa auriformis: Syft. nat. 12. p. 725. Fl. Juer. 1137. Sp. pl. I625.

Fungus mombranaceus auriculam referens $\int$. fambu. cinus, Baub. pin. $37^{2}$.

s.oc. Ijigna antiqua, præfertim fambuci. Infrequens. PHARM. AVRICVLAE JVDAE.

QVAL.

vis: refrigerans, exficcans, adntringens.

Trita, eximia. rsvs: Ophthalmia, Inllammatio, Angina.

COMP.

\section{LYCOPERDON.}

656. I.YCOPERDON Tuber globofum folidum mu: ricatum radice deftitutum. Fl. Suec. 128r. Sp. pl. 1655.

Tubera corvina. Baub. pin. 376 .

s.oc. Europx fylve dentix.

Subterreftris.

PHARM. HOLE'TI CERVINI.

QVAL. hircina.

vis: aphrodifiaca, flatulenta.

Exoleta, dubia.

vovs: Atecnia.

comp. Bals. apoplecticus. 


\section{CRYPTOGAMIA. FVNGI.}

657. LYCOPERION Bovifta fubrotundum laceratn: dehilicens. Fl. lapp. 525. Juec. 1280. Sp. pl.1053. Fungus rotundus orbicularis. Baub. pin. 374.

Loc. Europa campi ficciores. Vulgatifimus. WHARM, CREPI'T Y'S LVYI.

Qral. farinola, fyptica, nigricans. Trita, eximia. vis: adftringens, obftipans, abforbens, incraflans, gaftritica, opthalmica.

vsve: Amputatio, Hamorrhagia! Hamorrhois! Vlcera, Intertrigo.

cOMP.

657. Lycoperdon carcinomate Suput. pl. 453. in promontorio bonæ frei ad curanda ulcera cancrola adhibetur. Thmb. sised. afr.5. 


\section{㗊 距 \\ A P P E N D I X. \\ $P A L M A E$. \\ C.OCOS.}

6;8. Cocos butyracea inermis, frondibus pinnatis: folicolis funplicibus. Suffl. 454 .

Pindova. Pi\%. bral. 62. c. fig.

Loc. America meridionalis.

pHarM. PALMAE Cleum.

ejat. febacea.

VIs: emolliens.

vsvs: Pernio. [Durities ventris.]

\section{PIOENIX.}

659. PHOFNTX dagylifera frondibus pinnatis, folio. lis complicatis tilfiformibus. Sp. pl. 1655 .

Palma hortenfis. Kíemipf. anicen.6ó8.t. I. 2.

soc. India utraque. Aibor, fera. pharm. DAC'TYLI Fructus, Ona (Nuclei futius).

eral. dulcis, fubpinguio. lixoleta. vis: nutriens, corroborans, edulcorans, expectorans.

vsvs: Tufis, Raucedo, Stranguria.

cons.

\section{$F R V C T V S$. MYROB L A N I.}

560. MYROBALANVS indica nigra. Geolfr. mat 329. Dal. pharm. 334.

$$
\text { S } 5 \text { Pancl. }
$$

6g8. Non folum ex lace, fed coiam ex alis Palmamm furcic-

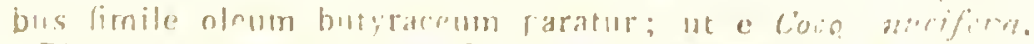

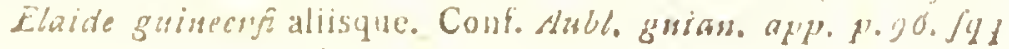


Panel. Rheed. mal. 2. t.9.

Loc. India orientalis.

PHARM. MY'ROBALANI INDAE Fruchus.

eval. Drupa oblonga, octangularis, rugola, dura, nigra, auftera.

Exoleta.

vis: adflingens.

vsvs:

comp. Hamech.

66r. BELLIRICA Myrobalanus. Breyn. ic. 18. t.4.

Tani. Rbeed. mal. 4. p. 13. t. 10. Decandra.

Loc. India orientalis.

Arbor, peregrina.

HHAM. MYROBAI.ANI BELLIRICAE Fructus.

Qval. Dmpa rotunda, camola, acris. Exoleta.

vis: adntingens, eccoprotica.

vsvs:

COMP.

662. CHEBVLA. Dal. pharm.334. Geoffr. mat. 328.

Myrubalanus Chebula. Vesl. cgypt.67.t.62.

A gialid. Alp. egypt. t. 39 .

LoC. India orientalis.

Arbor, peregrina. PHARM. MYROBALANI CHEBVLAE Fructus.

eval. Drupa quinquangularis, rugofa, nucleo car. nofo, fubacri.

Exoleta.

vis: adtringens, eccoprotica.

Vis: :

comp. Hamech.

663. CitrobalanvS. Dal. pbarm. 334. Geoffr. mat. 328.

Myrobalanus citrina. Pluk. mant. 132.

LOC. India orientalis.

ITARM.

66r. Alii lianc ftatuunt effe ab Idore mutli Rheed, Mala. 1.c. t.18. 363. Myrobalanos has quatuor ex eadem arbore Fanel Malabarorun, fed difpariami tempore, defunas effe dicit Adanfon. 
RARM. MYROBALANI CITRINAE Fructus.

Qval. Drupa pentagona, oblonga, citrina, ofliculo rugoro.

vis: adfringens, eccoprotica.

Exoleta.

vSrs:

comp. Hamech.

\section{$R E S I N A E$.}

664. AMMONiacvM. (An Paftinacx /p.?) Barbara. Loc. Lybia, Barca Aegypti occidentalis. PHARM. AMMONIACl Gummi-refina.

Cral. amaricans, alliata. Trita, eximia. VIS: refolvens, expectorans, diaphoretica, diureti-

ca, eccoprotica, emmenagoga, maturans.

vsvs: Hyfteria, Afthma! Obitipatio! Dyfuria, Tumores.

comp.

665 CARANNA. Dul. pliarm. 354. Geoffr. mat. 530. Flahveliloca quahvitl. Herm. mex. 56 .

zuc. Mexico.

PHARM. CARANNAE Gummi-refina, OO.

Qval. tenax, cinereo - nigricans, fubamaricans, fumo grato.

vis : refoliens, fomachica.

vsvs: Dulores Odontalgia, Arthritis.

comp. Emplattra.

666. MYRRHA. Dal. pharm. 355. Geoffr. mat.635. (an [Mimolx fpecies? Bruce.])

Loc. Regio Troglodytarum Africa. Bnhara. mHAR. MYRRHAE Gummi - refina $\theta_{i}, \mathrm{R} \mathbf{z} \beta$, ㅇ, Deliquizum.

QVAL. ruffa, anlara, fubacris, aromatica, naufeofa, graveolens.

Trita, eximia. 
vis: balfanica, tonica, antheiminthica, anciputredimola, flomachica, emmenagoga, vuisumia. vsvs: Hefleria, Jysnemorhar, Cachexia, Scor. butus, Gangrana, Vlcus.

comp. \%uch. mymbar, Andromach. Mithridat. Hhiloniun, Diateliaron, imef. hycinth. Pil.

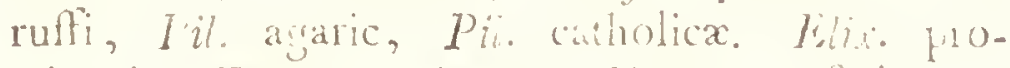
prictatis, I'iggu. martiqum, l'nger. apoflolormm, limpl. melilut, Emil. oxjerocems.

667. BDELLIVAr. Duil pbarm. 33r. Couffr. nal. 6az. Burbara.

r.oc. Arabia, Media, India.

PHARM. GLELLII COMmI-refina, 0

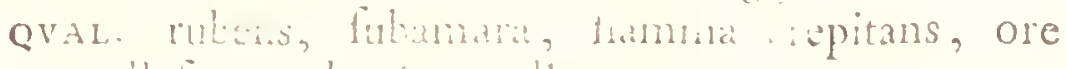
mollefous, dentious adharens.

vis: hislvens, emolliers, thoración, emmenago. ga, linaturans, vineraria.

vsis: Hyfteria, Hamombois.

есмн. Nithridat. Vngu. apefolonn.

608. SAGAPENVM. Dal. pliarm. 130. Geuffr. mat. 643. (Ferule fecies?)

Barbara.

coc. Alexandria, Perfia.

PHARM. SAGNIENI Gummi - refina.

Qvas, virula, acris, rufefens.

vis: ccceprotica, refolvens, pellens, emmenagoga! repellens, maturans.

vsvs: Hyfleria, Oblipatio, Afhma, Dysmenorrhora, Tumores.

comp. Andromach. Mithridat. Troch. myrrha. Empl.flicicum. Empl. diachyl. gurm.

669. COPA-

667. BDEI.LIVM Hermnnm!s in Lap. Iyd. 15 . judicat puritate

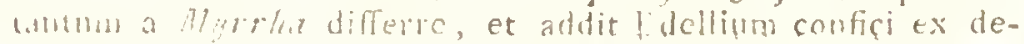

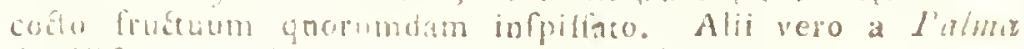
batolifera, in Arabia lelisi nalicente, deducunt. 
669. [COPAIIFERA. Berg. mat. 896 .

Copalli quahuitl, copallifera prima. Hernund. mex. 45$.

loc. Mexico.

PHARM. COPAL Refina.

QWA. toxica.

Arbor, manfueta.

Vis:

rsvs: Cephalalgia.

COMP.

Exoleta.

\section{MISCELLANEAE.}

670. AGALLCCHVM. Geoffr. mat. 213. Rumph. amb. 2. t.0. (Ficus fpecies?)

Lignum aloès. Dal. pbirm. 380. Kiffna. Kempf. anken. 903. t. g02. f.2.

toc. China.

Barbara.

PHARH. ALOES (AGALLOCHI VERI) Lignum

Ai. Refina, 0 .

eval. lactelcens, meditullium trunci amariflimum.

Rarior.

vis: venenatifima, excorians, incbrians, anthel. minthica.

rsvs: Chulera, Paralyfis, Podagra.

comp. Trocb. hedycroe, Conf. Alkerm.

671. ASPALATHYM. Dal. Ham. 379.

Agallochum prafantilinum. Baub. pin. 393. Ca. lambac. Dal. fine.m. 380.

Barbara.

Lor. India oricntulis.

PHARM, ASPNLA'THI Lignum.

QVAL.

Trita, eximia.

VIS:

VSVS:

669. Refina COPAL a fpecie Rhois defumi credita, fed fallio. Obf in mat. Hitd. 152. [Comal orientale fundit Elcosarpus ropallifora ketz, fufc, 4, p, 27. Kisnig.] 
vsvs: Colica.

COMP.

672. FERNAMBVCAEA.

Brafiliano fimile lignum, Vernimbock germanis dichum. Baub. bijt. 1. p.492. Barbara. r.oc. Brafilia.

PHARM. FERNAMBVCI Lignum.

Q'Al. rubra.

VIs: tincloria.

vsvs: mechanicus.

COMP.

[CALVMBA. Redi exp. 142. Semmed. Jimpl. 37. Cartbeuf. difj.136. Perciv. ed. 2. p.3. Berg mat. med. $570 . \quad$ Barbarb. Loc. Africa. Kenig. Retw. fn/c. 4. p.5.

PHARM. CALVMBAE (COLOMBAE) Radix $9 \beta .-3$ j. QVAl. amara, acriufcula, fubaromatica. Exinizo. vis: tonica, ftomachica, antileptica, antemetica. vsvs: Cholera! Dyfenteria, Colica biliofa, Febres biliof $x$, Morfura venenata.

COMP. 


\section{R E G N V M}

LAIDEWM 



\section{สล \\ CLASSIS I.

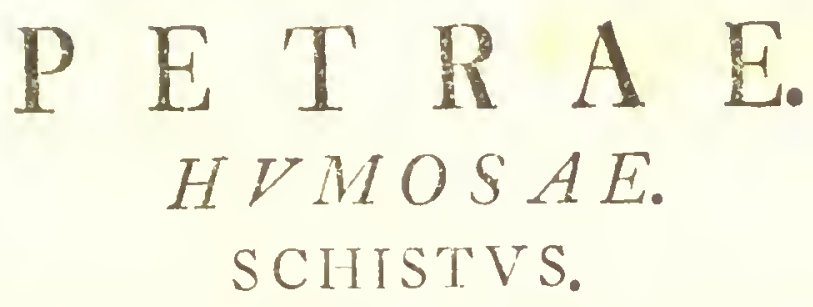

73. SCHISTVS Nigrua leriptura atra, ater inquinans. Sylt. nat. edl. 12. vol. 3. p.38.

Fiflitis mollior friabilis pictorius. Wall: min.67.

Luc. Hunneberg Weflrogothix.

PHARM. NIGRICA FABRILIS communis.

vis: terreftris.

vsrs: inechanicus.

COMP.

\section{$C A L C A R T A E$. MARMOR.}

674. MARMOR rude particulis granulatis micantibus. Syft nat. 4 I.

Calcarius particulis cintillantibus. Wall. min. 39.

loc. Runes in variis provinciis Suecix.

phar: CAlX VIVA, iota, Aqua.

vis: eflervefcens cum acidio. Vftulnta efferve-

fecris cum aqua, antacida, corroliva, venenata. vsvs: extermus, Vlcera.

roc.up.

\section{G Y PS VM.}

675. GIPSTM ufuale particulis arenaceis micantibus. Syit. nat. 45 .

Gyplum preticulis paralellepipedis et globolis concletum. Wall min. 57.

Loc. Polonia, Galdia, Germa tia, Livonia.

Pharia. GYYSVM preparatuin. 
vis : abforbens, antacida.

vsvs: interne venenatus.

COMP.

676. GYPSVM Alabaftrum particulis impalpabilibus, diaphanum. Sy/t. nat. 45 .

Gypfum particulis minimis punctis nitens, polituram admittens. Wall. min. $5^{\text {I. }}$

loc. extra Sueciam.

PHARM. ALABASTRVM preparatum.

vis: abforbens.

vsv's: antecedentis.

comp. Vngu. Alabafrinum.

\section{S T IRIVM.}

677. STIRIVM gypfeum pellucidum fixum fibrofum. Sy/t. nat. 47.

Gypfum filamentis parallellis compofitum. Wall. min. 49.

LOC. in Echifto Oelandix, Weftrogothix.

PHARM. ALVMEN SCISSILE.

vis: Alabaftri.

vsvs:

COMP.

\section{ARGILLACEAE. T A LC V M.}

678. TALCVM Rubrica fubfillile rubrum inquinans. Syft. nat. 5I.

Ochra rubra cretacea. Wall. min. 266.

loc. in Marmore et Cote inter rimas per Dalekarliam.

PHARM. RVBRICA FABRILIS.

vis: adnringens.

vsvs: mechanicus.

COMP. 
679. TALCVM Smectis ungue rafile, albo inqui. nans. Sy/t nat 52.

Talcum folidum femipellucidum pictorium. Wall. min. 129.

zoc. Garpenberg.

PHARM. TALCVM VIRIDE.

V1s:

vsvs: pictorius,

$\operatorname{con} 1$.

680. TALCVM Nephriticus præpoliendum viride fubdiaphanum, particulis fubfibrofis. Sy't nit 53. Gyplum viride femipellucidum fifile. Wall mir. $5^{2}$. zoc. Hilpania, Bohemia.

PHARM. LAPIS NEPHRITICVS preparatus.

VIS : dubia, ftomachica?

vsvs: Nephritis?

comp.

\section{A M A NTVS.}

681. AMIANTVS phmofus fibrolus, fibris feparabili. bus ilexilibus fragilibus pappofis. Sy/t. nat.55.

Asbeftus fibris parallellis fragiliflimis vix feparabilibus. Wall. min. 144. zoc. Fahluna, Dannemora. PHARM. ALVMEN PI,VMOSVM vis: interne venenata. Externe irritans. vsvs: externe Paralyfis.

cuMP.

\section{T C A.}

682. MICA talcofa lamellis thexuofis friabilibus virefecent-albidis diaphanis. Sy/t. nat. 59.

Talcum albicans, lamellis fubpellucidis, $W$ all. min. 127.

1oc. Verietir. 


\section{PHARM. TALCVM ALBVM.}

vis : cofmetica.

vis:

COMP.

\section{ARENATAE. \\ SILEX.}

683. SIl.EX cretaceus vagus, cortice cretaceo, fragmentis opacis Lavibus. Sy/t. nat. 67.

Silex comeus intrinfece xqualis, durillimus. Wall. $\min .77$.

LOC. Scania.

PHARM. SLLEX combuflus.

vis: irrelolubilis.

Finftranea.

vsve: intidus.

conp. Fel vitri.

684. SILEX Carneolus vagus diaphanus unicolor ruber. Syft. nat. 69.

Achates fere pellucida, colore rubefcente. Wall. min. 79 .

zOC. Sardinia.

PHARM. LAYIS CARNEOLVS, preparatus.

$v$ Is: antecedentis.

vsvs: antecedentis.

COMP. 


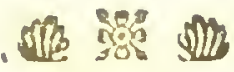 \\ CLASSIS II. \\ M I N E R A E. \\ $S A L I A$. \\ NITR VM。}

685. NITRVM natioum humofum. Syft. nat.84.

Nitrum terra mineralifatum. Wall. min. 165.

Loc. ubique in locis tectis.

PHARM. NITRVM COMMVNE. Depuratum $f$.

Crroftalli mineralss. Spiritus acidus, dulcis. Masnefir alba. Lapis prunelle. Irixi Liquor. Antimoiriatzim. Arcanum duplicatum.

vis: acida effentialis, refrige!ans, refolvens, ec-

coprotica, antifpasmodica, fphacelizans.

vsrs: Febres phlogifticx, Inflammatio, Hydrops; Anhma, Sitis, Stranguria, Hamorrhagia.

cosrp. Spec. pro ptifana laxativa Duriesi, Lapis medicamentofus, Antihedicum Poterii.

680. NITRYM Cryftallus montana lapidolum quart\%ofum ododccaediun hyalinum. Syft. nat. 84 . Crythilus hexagona non colorata. Wall. min.ro4. 1oc. in Saxis concavis.

PHARM. I.APIS CR YSTALLVS preparata.

vis: fiuftranca, noxia.

vsws: moxius.

cone. Pral\%. Lac provocans?

687. NITR TM Fluor Japidofum quartzofum octodecacitrum coloratum. Syst. nat. 85 .

a. - Hyacintbus purpureo-fulvum. 
Gemma plus minus pellucida, duritie nona, colore ex llavo rubefeente, igne ligueficens. Wall. min. p. I2I. $n \mathbf{I}$.

Loc. India.

PHARM. HYACINTHVS ORIENTALIS preparata. VIS:

VSVS:

comp Pulv. Bezoard. Semnerti, de Gutteta, Pannon. ruber, Sper. cordiales temperata, de Hya. cintho, Smaragd.

\section{B. - Anethyftus violaccum.}

Gemma pellucidilima, duritie fecunda, colore rubro in igne permanente. Wall. min. 115. 2.1 .

ıoc. Hilpania, Italia, Germania.

PHARM. AMETHYSTVS. ORILNTALIS preparata.

vis:

vivs:

COMP.

\section{N A T R V M.}

688. NATRVM fontamum nudum fontanum faturatum. Sy/t. nat. 89 .

soc. Epsham, Sedlitz, totus Oceanus.

PHARM. SAL CATHARTICVM ANGLICVM. [EHSOMENSE, SEDLIZENSE, SEIDSCHV. 'LENSE.]

688. Natri figuram perfede imitantur SAL MIRABILE Glanb. et POLYCHRESTVM PARISIINSE; cum autem prims a parte alkalina Salis comm. O). Vitrioli juneta, et polferius ab Acali Nitri et Acido Snlphuris conponontur, bec ordini fyetenatico inferere, ut artificialia, nolumus. [Sal mis:alile reperitur omuino nativam, partim folum in variis thermis, fontibus lacubusque falfis, mode excolum in yharmacopoliis profiat, Solis Thermonn Carolinarsen, SaJis 7 eplcufs, Salis Fridericiani aperitivi nomine; partion 
VIs: purgans, antacida, mundificans.

vsrs: Cacliexia, Quartana.

COMP.

689 NATRVM glaciale lapidofum gypfeo-fpatofum fufiforme pellucidum. Syft. nat. 90.

Gyplum lamellis rhomboidalibus pellucidum. Wall. min?. 4?.

Lor. Fodinx gyplex Gallix.

PHARM. LAPIS SPECVLARIS feu GLACIES MA.

RIAE.

VIs: abforbens.

vsvs: mechanicus.

COMP.

\section{B O R A X.}

69o. BORAX Tincal nudus. Syjt. nat. 94 .

Borax crudus cxrulefcens fexangularis. Wall. inin. IgI.

IOC. India orientalis.

PHARM. BORAX VENETA. Sal Jedativum.

vis: emmenagoga.

vers: Dysmenorlioca, Dyslochia.

CONP.

$$
\text { 'T 69т. BO- }
$$

coscrebun in formam pulveris vel cryftallorum, in lacubus Tisarixe autralis cxliccatis, Sial catharticum Aftrohanenf"; in fodinis falis bemme pallim cet.] SAL ANGLICANVMl obcineri potent ab omi Aqua marina, et non eft artificiale fod naturale Sal; videtur yuam proxime accedere ad naturan Salis mirabilis likath. Hinc Maloun contendit, Sal Anglicanum effe compofitum ox Sore mirabili et Sale com. mani; ac id ipfum in Gallia obtineri rave ac in Anglia. Ab aqua mariua Bothnica obtineri compertum eit $]$ a IV. [Sal anglicanus a milabili diverfilfinum, e maguelia alba et acido vitriolico conpolitum, fo utum in acidulis alfis. gue aquis, non vero in ommi aqua marina, ficcums cryfallinun in Schifto paffim reperitur, cujusinodi eft Sal aivisuss helvoticum cet.] 


\section{$290^{\circ}$ MIINERAE. SALIA.}

6gr. BORAX Gemma nobilis lapidofus pifmaticus pel. lucidus; pyramidibus truncatis. Syjt.nat.94.

a. - Topnzius Havis.

Gemma pellucidifima, duritic quarta, colore au. reo, in igne permameite. W'all. min. In.

I. ox: in Fodinis $\geqq$ etallicis.

PHARM. TOPASIVS prepparata.

VIS:

VSIS:

comp. Species et Confectio de Hyacintho. B. - Smaraglus viridis.

Gemma pellucidatina, duritie quinta, colore viridi in igne non permanente. Wall. min. Irt. Loc. J'eruvia.

PHARM. SMARAGNVS ORIENTALIS praparatus. vis: fuperflitiolia, infida.

vovis:

comp. Confect. Smaragdi, Specif. cephalicum, P,lv. Be\%ord. Sennerti, Pannon. ruber, Spec. de Hyacintho cordiales, temperatx.

692. BOR AX Granutus teffellatus folidus politus fcintillans. Sy/t. nat. $9^{n}$.

Gemma plus minus pellucida, duritic oflava, colore obleure rubro, in igne permanente. Wail. min. 120.

Loc. in Fodina nova Cuprimontana Suecia. yHARM. CiRANATI'S preparatus.

vis: dubia.

vers: inlidus.

COMP.

\section{V RIA.}

693. MIVRTA marina nuda marina. siyft. nat. 98.

Miuria marina. Wall. min. I6g. 
Ioc. Mare, prefertim regionum calidarum. PHARM. SAL COMMVNE. Spiritus, acidus, dulcis. vis: Homachica, aphrodifiaca, calefaciens. vs's: Stomacace decrepitatum. Yerniones $\Omega$. COMP.

60.4. MURIA fontonn nuda fontana. Syft. nat.98. Muria fontana. $H^{\top}, l l l m$ rm 80.

Loc. Fontes falfi pafim per Europam. PHARM. SAL COMMIVNE.

VIS: pracedentis. vsrs: precedentis. comp.

695. MVRTA montnma nuda foflilis. Syft. nat. 98. Muria forlilis pura. Wall. min. 168.

Loc. Polonia, Hungaria.

FHARM, SAI, GFMMAE.

VIS: antecedentis.

vsus: aconomicus.

conp. Elect. Benediot. laxativum, Pulv. ad fuppo. fitoria, Vngu. de Arthanita.

\section{A M MONIACVM.}

6g6. AMMONIACVM fal. Wall.min. 187.

uoc Italia, [Calmuckia.]

pHar.s. SAl. AMMONIACVM. $\Omega$ volatilis, acidus. vis: incidens.

rsis: lictores.

comp.

\section{$\Lambda \mathrm{L}, \mathrm{V} M \mathrm{~F}, \mathrm{~N}$.}

697. AI.VMEN commume Schilli. Syft. nat. Ior. T5

633. Spritus Salis dule is cum chatla cmporetica caute appli-

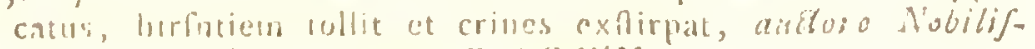

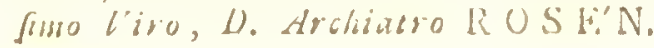


Alumen lapide fifili mineralifatum. Whall. min. I7j. Loc. Scania, Oelandia, Weftrogothia.

pharm. ALVMEN CRVDVM, VJlum, Spiritus. Phlegma.

vis: acida, coagulans, adntringens, fophificans. vsvs: exterm. Stomacace, Prolapius, Sarcoma, VI. cus, Hemorrhagia, Ophthaimia.

comp. Lapis medicamentofus, limpl. de Spermate Ranarum, Tindt. Gummi Laccix, Vngu. Aegyptiacum, pulv dentifricii.

698. ALVMEN romanum Mamoris. Syft. nat. roz. Alumen lapide calcario mineralifatum. Wall. min. 174 . Loc. Italia.

PHARM. ALVMEN ROCHAE.

vis : antecedentis.

vis: rarior.

COMP.

699. ALVMEN Gemma pretiofa lapidofum pellucidif. fimum folidifinum. Sy/t. nat. 102.

a. -. Rubinus rubrum.

Gemma pellucidillima, duritie fecunda, colore rubro in igne permanente. Wall. minn. III.

Loc. Infula Leylona.

PHARM. RVBINVS ORIENTALIS preparatus.

VIS:

VSVS:

comp. Pulv. Pannonicus ruber, Bezoard. Sennerti. B. - Sapphinus caruleum.

Gemma pellucidifina, duritie tertia, colore $c x$. ruter in igne fugaci. Wall. min.ru.

Loc. Silefra, Bohemia.

PHARM. SAPHIRVS ORIENTALIS preparata.

Vis:

VSVS:

COMP. 
- comp. Spec. Cordial. temperatx, de Hyacintho, Pilv. Pannon. ruber, Conject. Smaragdi, de Hya. cilitho.

\section{VITRIOLVM.}

700. VITRIOLVM martis ferri viride hexaëdrum. Sy/t. nat. I04.

Vitriolum furri viride nativum. "Wall. min. I66.

Loc pafim in Pyritis et Fodinis ferri.

pharm. VITRIOLVM MAR TIS COMMVNE, Tartanus vitriolutus, Tacbenii. Holmenlis. Colcotirar.

vis: acida, coagulans, anthelminthica, vomitoria, aditrince iis.

vsvs: Hamorrhagia, Vlcus, Febres putrida.

comp. I'iulce de gummatibus, Pulv. ad vermes.

7or. VITRIOLVM cyprizin cupri cæruleum dodecaëdron. Sy/t. nat. ro4.

Vitivlum cupri caruleum nativum. Wall. min.165. soc. e Cypro adportatur.

PHAR U. VITRIOI.VM CYPRIVM.

vis: corrofiva, coagulans, cathretica. Externa. vsis: extem. Homnorhasia, Vulnus, Sarcoma. comp. Lapis medicancntolus, Pulv. fympath. Digb.

702. VITRIOLVM alhum zinci album dodecaëdron prifinaticum. Sy/t. nat. ro4.

Vitrinim zincs album nativum. Wall. min.167. Loc. Hircynia.

PHARMT.

700. Vitriolum Martis artificiale e I.imat. Ferri et Ol. Vitrioli praratur, Solutione et Cryfallifatione.

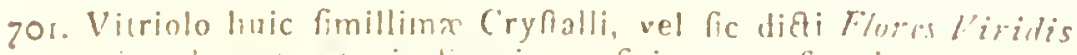
achis, th aceto et rindt: arris conficiuntur, folutione et ciyfallifatione, colore faturato viridi gaudentes. 
PHARA. VITRIOI_VM ALPVM.

vis: adlringens.

vsve: Ophthalmia.

conp. Empl. Diapalma, de Spermate Ranarum, Sticticum, Theriaca Andromachi.

703. VITRIOLVM plumbi album;

urc. Sponte in Fodinis plumbi; sed parcifime, binc ars illud imitatur.

IHARM. SACCHARVM SATVRNI.

vis: exficcans, obtundens.

vssis: Erylipelas, Ophthalmia, Vlcus, Gonorlioa. comp. Empl. Opodeldoch, Vngu. Nihili.

\section{SVLPHVRA. \\ AMBRA.}

704. AMBBR A ambrofiaca grifea. Syft. nat. ror. Rumpb. mif. 262. $t \cdot 53.54$.

Anbra variegata. Wrall. min.204.

roc. Mare, profertim India orientalis.

PHARM. AMBRA GRISEA, EDentia.

VIs: ambroliaca, nervina, analeptica, aphrodifia-

ca, cxcitans, a contagiis profervans.

rsus: Scabies, Debilitas, Hypochondriafis.

comp. Elix. vitx, Balfan. Apoplect. Confect. Al. kermes conpl., de Hyacintho, Pulv. Cachechi. cus, Spec. Aromat. rofatx, Cordiales temperata, de Hyacintlo, Troch. de Gall. Molichata, Mnga ad formacem.

705. ANISRA vulgatior unicolor fufco-nigricans, Sy/t. nat. 107 .

Ambra

903. A Calce Plumbi et Aceto denillato obtinetur, cotione et

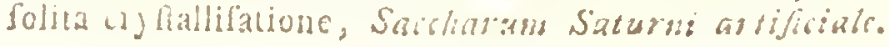


Ambra unicolor albefcens, citrina fufca 1 nigra. Hall. min.205.

Loc. pracedentis.

PHARY. AMBRA NIGRA.

$v i s:$ pracedentis, fed odore inferior.

vsis: pracedentis.

comp. priecedentis.

\section{S V C CIN VM.}

706. SVCCINVM electricum. Syft. nat. ros.

Sucinum pellucidum aut opacum. $W_{\text {all. }}$ nin. 200. $20 \mathrm{I}$.

soc. Mare Balticum.

PHARM. SUCCINVM ALBVM vel CARABE CITRINVM, preparat. Sal, Oleum, Balfam. Tincitura.

vis: calefaciens, ficcans, roborans, antihyfferica! fudorifera, emmenagoga.

vsrs: fumus in Catarrhis.

conp. Empl. Diaphoret. Opodeldoch, Catagmaticum, Sticticum, Spec. Cephalicum, Pulv. Bezoard. Sennerti Cachecticus, pro Epithemate cordis, Species Diamofchi dulces, Trocbifci Alkekengi, de Carabe.

\section{BIT VMEN.}

707. PITVMEN Petrolen liquidiufculum fufco-rum felcens. Sy/t. nat. rog.

Pitumen liquidum craflius obfcure brunneum. Wall. min. 194 .

Loc. Rattwik Dalckarlix.

PHIRM. HETROLEVM f. OLEVM PETRAE

NATIVVM rubrum, album.

vis: expectorans. 
vsvs: Colica Pictonum! Dolores ofteocopi, Txnia! comp. Empl. carminativum.

708. BITVMEN Mumia Subfriabile piceum. Sy/t. nat. IIo.

Mumia mineralis. Hafjelq. it. 537. Kempf. anœer. 516. LOC. Perfia.

Pharm. MVMIA PERSICA nativa.

vis: vulneraria!

vsvs: rariflimus.

COMP.

809. BITVMEN Afpbaltum friabile atrum. Syft nat. I05.

Bitumen folidum coagulatum. Wall. mmn.195.

roc. Oelandia.

PHARM. ASPHALTVM feu BITVMEN JVDAI-

CVM. [Oleum deftillatum.]

VIS:

vsvs: extern. Vlcera fordida. [Phthifis o o.]

comp. Theriaca Androm., Trocb. Cypheos pro

Mithridatio.

\section{PYRITES.}

710. PYRITES nativns nudus diaphanus. Syft. nat. niz:

Sulphur nativum purum llavum. Wall. min.205.

LOC. Rullia.

PHARM. SVLPHVR VIVVM.

vis : fequentis.

vsvs: fequentis.

COMP.

7Ir. PYRITES Ferri mineralifatus amorphus fintil. lans. Syft. nat. 115.

Sulphur ferro mineralifatum, minera difformi pal. Lide flava nitente. Wall. min. 208. 
Loc. Dyita Nericix.

PHARM. SVLPIHVR CITRINVM, Oleam Stillatitiun, Spiritus per campan. Grifeum v. Cabali Hepar, Tinctura, Flores, Balfamm, Terra rubra, Lac. ris: expectorans, diaphoretica, inquinans, ecco. protica.

vsrs: Tullis, Scabies.

conp. Sulplurata. Balf. Anifatum, Foniculatum, Juniperatum, Terebinthinatum, Succinatum.

712. PYRITES Auripigmentum nudus flavus micis auratis. Sy/t. nat. mis.

Arfenicum fulphure, lapide fpatofo et micaceo mineralifatum, minera Havefcente. Wall. min.226. coc. Hungaria, India orientalis.

PHAR. AT. AVRIPIGMENTVM.

vis : mechanica.

vsvs: pictorius.

COMP.

\section{ARSENICVM.}

713. ARSENICVM mulim nudum cryftallinum purum. Sy/t. nat.ir?.

Arienicum album. Wall. min. 223.

Loc. Germanía.

PHARM. ARSENICVM album, Citrinunn.

vis: maxime corroliva. (Preftat non uti).

vsvs: Febr. intermittens, noxius. [Carcinoma.] COMP.

714. ARSENTCVM Sandaraca nudum rubrum. Sy/l. nat. II7.

Arfenicum fulphure mixttm rubrum. Wall. min. 224.

L. OC.

713. Arfenicum quatenus in aqua folvitur et cryfallifatur, Salibus; quatenus acido conftat et plilogifo, Sulpluribus; quatenus regulum dat, Metallis adnumerari poteft.] 
roc. Hungaria, Turcia.

PHARM. ARSLNICVM Rubium.

vis: corrofiva.

viss: noxius.

cOMP.

\section{METALLA. HYDRARGYR VM.}

715. HYDRARGYRVM virgineum nudum fluidum. Sy/f. nat. 119 .

Hydrargyrum nativum. Wall. min. 218. Loc. I fungaria.

PHARM. NIERCVRIVS O. ARGENTVM VIVVM.

1. depuratumn.

$$
\text { PRAEPARATA. }
$$

2. Aetliops min. fit ex 1. et Sulphure, iritratione.

3. Mer. Sublim. comoliv. ex I. Ayura forti et Sale comm decrep. Sublimitione.

4. - dulsis: ex 3. et Miercur. vivo, tripla Siblimitione.

5. Merc. pracilit. albus: ex I. in Aqua forti boluto, rreeifitutione c. Sale muriatico.

6. - ruber: priori Solutione evipurats, re. verleretur refidumin.

7. - viridis: ex 1 . et Cupro in Aqua forti fulutis, evaforatione et digefizune $\mathrm{c}$. aceto.

8. - - luters $\int$ Trorpethum miner ale: ex x. et Ol. vitrioli, Solutume at pire.ipla tione c. Oleo tartari per deliqu.

9. Arcan. Corllmum: ex 6. et Alkohnl vini quadrupl.

10. Panacea Limma, ina: ex 4. ochies iterata Sibblimativire. 
vis: fralagoga! anthelminthica!

vsrs: Syphilis, Infecta, Scirrhus.

comp. Vingu. Pediculorum, Emplaftr. de Ranise. Mercur.

716. HYDRARGYRVM Cinnabaris mineralifatum pyriticolum fibrofum. Sy/t. nat. IIg.

Hydrargyrum fulphure mineralilatum, minera rubra. WVall. min. 219.

Loc. Hungaria.

PHARM. CINNAPARIS NATIVA, preparata. vis: Hydrargyri; fed minus Colubilis.

vsrs: antecedentis.

Ecmp. Specific. cephalicum.

\section{MOLYBD AEN VM.}

717. MOLY'BDAENVM Plumbago tritura cxrulefiente. Sy/t. nat. 1ar.

Mica pictoria nigra manus inquinans. Wall. min. 26. Loc. Finlandia.

PHARM. PLVMBAGO.

vis: mechanica.

vevs: pictorius.

cons.

\section{S T IB I V M.}

718. STIBIVM friatum mineralifatum fibrofum plumbicolorum. Sy/l. nat 123.

Antimonium fulphure mineralifatum. Wall. min. 238. LOC.

716. Cinnabaris Antimonii poft defillationem Butyri, auito igrsis gradu oldinesur; Cinnabaris vero factitia, a Mlerc. viv. et Sulphure fublimulur.

717. [Plumbarso ad Sulphura referenda non ad Metalla, proprii generis non Molybdani eft, ex obfervat. Burgmanni et Sinculii.] 
zoc. Gallia.

PHARM. MINERA ANTIMONTI Hungarica.

I. ANTIMONIVM CRVDVM.

PRAEPARATA.

2. Antim. Butyrum: ex Mercur. fublimat. corrofivo et $\mathrm{I}$. defillatione.

3. - - Regulus: ex 1. Nitro et Tartaro liquatis, coni fuforii vibratione fubfideat.

4. - Sulpbur auratum: ex Reguli praced. Scoriis, in Aqua coclis, precipitatione cum Acido.

5. - - Flores albiff. ex 1. in Aqua Regis foluto et precipitato Aqua pura.

6. - Lilores rubri: ex I. et Sale Ammon. precipitatione et lotione Aqua.

7. - - Clyfus $\int$. Spirit. ex r. Nitro et Sulphu. ie, defillatione.

8. - - Diapboreticum : ex r. et Nitro, detonatione.

9. - Hepar: ex r. et Nitro.

Io. - - Regulus Martialis: ex I. et Ferro, liquatione et retonatione cum Nitro.

11. - Diaphoret. Mart. Ludov. ex 1. et Ferro, liquatione detomatione cum Nitro at edulcoratione.

12. - Vitrim: ex r. calcinatione.

13. - Tinctura: ex anteced. et Spiritu reruginis, digeftione.

I 4. - Cerufla: ex 3. et Nitro, detonatione et edulcoratione.

Vis: evacuans, pellens.

vsve: [Arthritis, Dolor ofteocopus, Vlcus, Scabies, Lippitudo.

comp. Mercurius vitec correstus: ex 2. Aqux purx inflillato et jubjolentia praccipitato. Bezoarticum minerale: ex 2. vel 4. et Spiritu Nitri, digeffione, 
evaporatione et reverberatione. Crocus Metallorum: ex 9. coetione in Aqua pura et precipitatione aceto vini. Aqua Benedicta Rulandi: "ex 12. et vino, infufione.

\section{ZIN C V M.}

7rg. ZINCVM mineralifatum mineralifatum compa: ctum fubfquamolum. Sy/t. nat. i25.

Zincum fulphure plumbo $f$. ferro mineralifatum ob. fcurum, particulis micantibus. Wall. min. 247. Loc. Rættwik Dalekarlix. PHARM. ZINCVM, Flores, Nibilum allum. vis: (Flor.) [antifpasmodica.] externe exficcans. vsvs: Vlcus, Lippitudo, Intertrigo!

COMP.

720. ZINCVM Calaminaris fubterreum lapidefcens: Sy/t. nat. 126 .

Zinci minera terrea, colore flavefcente f. fulca. IV all. min. 247.

roc. Polonia, Hungaria.

PHARM. LAPIS CALAMINARIS f. CADMIA FOSSILIS, Tutia.

VIS : antecedentis.

vsvs: Eryfipelas externe.

comp. Empl. de Lap. calaminari, Opodeldoch, de: fenfivum, ficticum.

\section{VIS M V T V M.}

721. VISMVTVM nativum nudum. Sy/t. nat.128.

Vismutum nativum. Wall. rim. 242.

Loc. Germania. 
PHARM. BISMVTVM f. MARCASITA, Magifterinim.

vis: dubia; (pracipitati) colinetica.

vsvs:

COMP.

\section{COBALTVM.}

723. COBALTVM arfenicale ferro et arfenico metaliiformi mineralifatum. Sy/. nat.rag.

Cobalti minera diverfimode Ggurata. Wall, min. 226. roc. Los Helfingix, Saxonia.

FHARM. CADMIA METALLICA f. COBALTVM, Cerulequn factitium. Smalumn.

vis: Arfenici, debilior.

vSVS:

COMP.

\section{STANNVM.}

723. STANNTM cryftallinam tefferis cryfallinis. Syft. nat. 13o.

Stanuum ferro et arfenico mineralifatum, minera cryftallifata polyëdra. Wall. min.202.

zoc. Persberg Suecix.

PHARM. S'TANNVM Anglicanum, Bezoarticum joviale.

v1s: anthelminthica?

vsvs:

comp. Antihecticum Poterii, Aurum et Argencium mufivum.

\section{PL V MB V M.}

724. PLVMBVM nativum nudum. Sy/t. nat. 132.

Plumbum nativum. Wall. min. 272.

LOC.

722. Madinia motnllica frepius eft Arfenicum metallicum feu Z. gulus Arfenici.] 
I.OC. Germania.

I'AAR. PLVMBVM, uftum, Ocbra factit. Lithargy-

rium: Auri, Argenti, acet. Ceru/fa, Minium. vis: exliccans, obtundens, adfringens, obftipans. vsis: Dolor ofteocopus, Rheumatifmus, Vlcus. comp. (uftum) $V$ ng $u$. de Tutia.

\section{FERR V M.}

735. FERRVM Selectun retractorium nigrans, particulis Subimpalpabilibus folidelcens. Sy/t. nat.r37.

Ferrum mineralifatum, minera cinereo-nigra, ma. gneti amica. Frall. min. 256. n. I.

ц oc. Dannemora.

PHARM. CHALYBS. Limatura. Crorus adfringens, aperitious. Preparatzs, timctura aperitiva. Scorioe. prep. Sapa.

vis: tonica, adltringens, antacida.

vsvs: Cachexia, Hypocinondriafis, Diarrhœa, Chlorolis, Dysmenorhaca.

COMP.

726. FERRVM Smiris retractorium rubricofum vitrum arans. Sy/t. nat. 139 .

Ferrum mineralifatum, minera duriflima rapaci fo.

lida magneti retractoria. Wall. min. 267 .

Loc. Europa aufralior.

PHARI.. LAPIS SMIRIS.

VIS:

vsvs: mechanicus.

(:) MP.

.727. FERRVM Hematites intractabile rubricans glandulolum, fragmentis concentratis. Syft. nat. 140 . Ferrum mineralifatum, minera figurata rubra $f$. - tritura rubente. Wall. min.249. 
Loc. Anglia, Germania.

PHARM. LAPIS HAEMATITES, proparatus. VIS:

vsvs: Febres intermittentes.

сомт. Emplaftr. ad Rupturas, Sticticum.

728. FERRVM Magnes attractorium. Sy/t. nat. I42.

Ferrum mineralifatum, minera ferrum trahente ac repellente et polos mundi oftendente. Wall.min. 262. $n$. I.

xoc. 1.apponia, Gagne.

Pнат.1. LAPIS MAGNES, preparatus.

vis: Ferri.

vsus: Colica?

comp. Empl. Sticticum, Opodeldoch.

\section{VPR VM.}

729. CVPRVM nativun nudum minerx inhærens. Sy/f. nat. 843 .

Cuprem nativum granulatum. Wall. min.276.

Loc. Klcwa Smolandix, Riddarhyttan.

PHarm. AES, Squamx, Crocus, Vflum, Viride, Spiritus. vis: corrofiva, interne caute.

vsvs: Ophthalmia.

comp. Aqua ophthalmica fapphirina Scbrcederi.

730. CVPRVM? Lazuli caruleum fcintillans. Syft. nat. 145 .

Jafpis colore caruleo et albo mixto, cuprifer. Wall. mini. 9 I.

Loc. Nía et Africa.

PнARм. LAPIS LAZVLI, preparatus, Vltramarimum.

VIS :

VSVS:

comp. Confectio Alkermes. 


\section{MINERAE. METALLA.}

73I. CVPRVM Armenus caruleum calcarium. Syjt. nat. 1. 6.

гoc. Europa auftralis, Armenia, Hungaria.

pharur. LAPIS ARMENVS verus preparatus, Ceruleum montanum.

VIS :

vsvs: mechanicus.

COMP.

\section{AR GENT V M.}

732. ARGENTVM natioum nudum. Syft. nat. 148. Argentum nativum. Wall. min. $30 \%$.

LOC. Prattfors Wermelandix.

PHARM. ARGENTVM foliatum, in conchis, Las jis infernalis, Besioard. Lunare, Tinctura Lunae. vis: politica.

vsvs: Oeconomicus.

comp. Species cordiales temperatx, de Hyacintho; Corfeit. de Hyacintho.

\section{A VR VM.}

733. AVRVM nativum nudum. Syjt. nat. 15r.

Aurum nativum. Wall. min. $32 \pi$.

Loc. Smolandia.

rHARM. AVRVM foliatum, in concbis, Fulmi: nons, Bezoardicum folare.

vis: antecedentis.

vsvs: antecedentis.

comp. Consect. de Hyacintho, Pulv. Bezoard. Sen: nerti, Epilcpticus, de Gutteta, Species de Hya. cintho, cord. temper. 
CLASSIS III.

\section{F O S S I L I A. PETRIFICATA. HELMINTHOLITHVS.}

734. HELMINTHOLITHVS Fudaicus obovatus cre. nulato - ftriatus fubpetiolatus. Syft. nat.165.

Lapis judaicus. Imperat. nat. $745 \cdot f .1-4$.

Loc. Scania.

PHARM. LAPIS JVDAICVS, preparatus.

vis: Spati.

YSTS:

COMP.

735. IIELMINTHOLITHVS Belemmites Alcyonii Lyncurii. Sy/t. nat. 170 .

Lapis lyncis. Gesn. lut. 92. Rampb. muf.t.50.f.I-5. LOC. Europa auftralis, Scania.

PHARM. LAPIS LYNCIS f. LYNCVRIVS, preparatus.

vis: cuicaria.

rsvs: exoletus.

COMP.

\section{CONCRETA. CALCVLVS.}

736. CALCVIJS Bezoar gaftricus folidus tunicatus. Sutr. iat. 1\%б.

Caknlus animalium caprini generis, cruftaceus. it all, min. 4 in

loc. Ventriculi piica in Capris. 


\section{FOSSILIA. CONCRETA.}

PHARM. LAPIS BEZOAR $\alpha$ ) ORIENT. Tinctura. B) OCCIDENTALIS.

vis: diaphoretica, alexipharmaca.

vis: Febres exanthematica.

conp. Pulv. Hezoard. Semerti.

737. CALCVLTS fellis felleus. Syft. nat. 177.

y Erinacei malaccenfis.

Lapis malaccentis. Ciuf. exot.217.

Loc. India.

PHAR.M. LAPIS PORCINVS R. MALACCENSIS.

Vis:

vsvs: Icterus.

738. CALCVLVS Margarita conchylii. Sy/t. not. I77.

Calculus animalium concharum. Wall. min. $4 \mathrm{iz}$.

Loc. Teftx Myx margaritifice amd noftrates.

PHARM. MARGARITAR $\alpha$ ORIENTALES.

B) OCCIDENTALES.

vis: calcaria, abforbens.

vsis: Acidum fpontaneum.

cusp. Saccharum P'erlatum, Confect. de Hyacin. tho, Sprcif. Cephal. Pulv. Bezoard. Semerti, Cacheclius, Epilepticus, Pannonicus ruber, $S_{p e-}$ cies cordiales temp. de Hyacintho, Diamargaritar. frigid. Diamofchi dulces, Diarrhodon. Abbatis.

\section{TARTARVS.}

739. TARTARVS vimi vini. Syft. nat. 178.

Tartarus. Wall. min. 1;r.

LOC. Dolia vini.

pharm. TARTARVS, Albus, Ruber, Spiritus, Oleum deft. p. deliqu., Crenur, Calcinat. ad albed. Tartarifat., Terra foliata.

737. [Vel potius Hyftricis criffata]. 


\section{I4 FOSSILIA. CONCRETA.}

vis : acida, eccoprotica.

vsvs: Fobris calida.

comp. (Cramor). Paliula laxativx, Pulv. polychren. de tribus, Species diajalappix, Syrupus Fol. Sennx.

\section{AETITES.}

740. AETITES aquilinus embryone libero lapillulofo. Syft. nat. 179 .

Lithotomi cavitate latente donati. Wall.min. 40\%.n. I. Loc. Helvetia,

PHARM. LAPIS AETITES I. AQVILAE.

vis: fuperftitiofa

vsvs: Partus difficilis?

COMP.

\section{PVMEX.}

74r. PVMEX Vulcani fchifti niger. Syft. nat. I3T.

Porus igneus lapidis Asbefti. Wall. min. 328.

LoC. Montes ignivomi.

PHARM. LAPIS PVMEX.

vis: mechanica.

vsvs: Tartarus dentium.

conip. Pulv. ad dentifricia.

\section{TOPHVS.}

742. TOPHVS Ofteocolla calcarius fubcylindricus perforatus. Sy/t. nat. IS9.

Petrificatum vegetabile radicis arborum vel plantarum. Trall. min. 335 .

Loc. Marchia Electoralis Pruffix nova.

IHARM. OSTEOCOLLA cruda, preparata. 


\section{FOSSILIA. TERRAE.}

Yis : adtingens.

vsrs: Fracturx.,

COMP.

\section{$T E R R A E$. \\ O CH.RA.}

743 OCHRA Ferri ferri pulverea lutea.' Sy/t. nat. Iga. Ferri terra pracipitata non mineralifata. Wall. $\min .205$.

Loc. ubique ad acidulas et in paludibus. PHARM. OCHRA NATIVA f. FOSSILIS.

vis: adfringens, exficcans.

vsvs: externus.

COMP.

744. OCHRA Aeris Cupri pulverea viridis. Syft.nat. I92.

Cuprum folutum et corrofum precipitatum viride. Wall. in in. 278. n. 3.

LOC. ad Fodinas cupri frequens. PHARM. VIRIDE MONTANVM.

vis: cupri.

vsis: pictorius.

CUMP.

\section{ARGILI.A.}

745. ARGIILA Temmia incarnata fracturis glaberrimis. Sy/t nat 2or.

Terra lemnia. Dal. pharm. 2o. n.2.

uoc. l.emmus.

PHARM. T'ERRA IEMNIA.

vis: plaftica, exficcans.

vsvs: exoletus. 
comr. Elect. Diafcord. Tberiaca Androm. Confect. de Hyacinth.

746. ARGILLA Tripolitana feabra nitidula flavefeens inquinans. Syjt. nat. 202.

Glarea indurata coharens appera. WVall. min. 33.

Loc. in Fodinis Mauritanix.

FHARM. 'TERRA TRIPOLITANA.

VIs: ablurbens.

vs's: mechanicus, Intertrigo.

costs.

747. ARGILLA Bolus ore liquefcens. Syjt. nat.203. Argilla pinguis. Wall. minr. 19.

a. - alba.

Bolus alba. Dal. pharm.19. n.8.

Loc. Goldberga, Turcia.

Prar.r. HOLVS ALBA; Terra Jigill. alba S. Meliteny. Turcica.

B. - Nava.

bolus ilava.

Loc. Silefia.

1HAm. TERRA SIGILLATA CITRINA, Silefaca f. Strigunzanfis.

$\gamma .-$ - rubra.

Jolus armena. Dal. pharm. 19. n.r.

soc. Peria, Armenia, Gallia, Germania.

IIARM. BOLVS ARMENA.

d. - viridis.

RVBRA. Terra figillata rubra.

Loc. Sinyrna.

pHarm. TERRA VIRIDIS.

vis: pla!tica, exficcans.

vsvs: cxolutus. 


\section{FOSSILIA. TERRAE.}

Comp. y) Elect. Diafcord. Cerat. Santalin. Spec. et Confect. de Hyacinth. Pulv. Dyfent. Pamnon. rub. Trochifc. Alkekengi. Empl. Cataginat. Lapis me. dicamentofus.

748. ARGILlA Vmúra humora fufea inquinans. Syft. nat. 204.

Vmbra fufca. Wall. min. 9.

Loc. Europa.

PHARM. VMPRA f. VMBRICA.

B. Vmbra obfcure fufca. Wall. min. 8.

Loc. Colonia.

PHARM. TERRA COLONIENSIS.

vis:

VSVS:

COMP.

\section{ALX.}

749. CALX Creta folubilis impalpabilis cohxrens. Syft. nat. 206.

Creta cohrens folida, Wall. min.8.

ıoc. Montes Gallix, Anglix, Danix.

pharm. CRETA, Pulverifata, lota.

vis: calcaria, abforbens.

vsus: Acidum fpontaneum, Soda.

COMP.

750. CALX Gur fixa impalpabilis farinofa. Syft. nat. 207.

Creta friabilifima leviflima non cohxrens alba. IVall. min.II.

Loc. Germania, Helvetia.

PHARM. LAC LVNAE f. STENOMIARGA. 
318 FOSSILIA. TERRAE.

vis: abforbens, lactifera.

vSvs:

COMP.

\section{HVMVS.}

751. HVMVS damafcena vegetabilis ochraceo - rufa. Sy/t. nat.2II.

Humus rubra obfcure rubefcens. Wall. nin. 8. LOC. Anglia.

PHARM. TERRA ANGLICANA.

VIS:

vsvs: pictorius.

COMP.

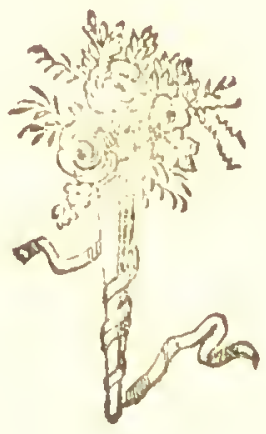




\section{N D E X}

\section{$\begin{array}{lllllll}G & E & N & E & R & V & M\end{array}$}

A A.

$\begin{array}{lll}\text { A canthus } 444 & \text { Anemone } 393 & \text { Balana } 25 \\ \text { Achillea } 533-540 & \text { Anethun 229.230 Bellis } 53 \text { L }\end{array}$

B.

Achras $26 \mathrm{r}$

Acipenfer $4 \mathrm{I}$

Aconitum 386.387 Angelica 214. 215 Berberis 262 Anthemis 535-536 Beta ro4 195

Acorus 260

Actaca $36^{2}$

Adiantuin 642

Aethufa $2 \approx 2$

Aëtites 740

Agrimonia 335

Ajuga 400

Alcea 456

Alcherilla 127

Allamanda 182

Allium $2+9.25^{2}$

Aloẽ 250

Althaca 465

Alumen 697-699

Ambra 704. 705

Amiantus $68 \mathrm{r}$

Ammoniacum 696

Amomum $70 \cdot 72$

Amygdalus 344.345

Anyris 273. 274

Anacardium 293

Anagallis $\mathrm{r}_{42}$

Anagry yis 297

Anas 28.29

Anchufa 132. 133

Antirrhinum 437

Apis 52

Apium 234.235

Açuilegir 389

Aranea 54

Arbutus 3i7

Aretium 507

Argentum 732

Argilla $7+5-7,48$

Ariftolifica $457-460$ Ariftolochia $552-556$ Bromelia 248 Araica 529

Arfenicum7r3.7I4 Bubon 218.219

Artemifia 516 Buxus 563

Arum 558

A farum 33x.

Afclenias 186. 187 Cæálpinia 303305

A fparagus 256

Afperala 115

Arphodelus 255

Afplenium 636

Aftragalus 489

Athamanta 207

Atriplex 622

Bupleurum 203

C.

Calculas $727-729$

Calendula 545

Calx $749.75^{\circ}$

Cambogia 376

Camphorofona 126

Cancer 56. 57

Canella 332

Atropa 163.164. Canis $4-6$

Aurum 733

Cannabis 602

Aveua 108

Capparis $37 \mathrm{~K}$ 
INDEX GENERVM.

Capra I8

Capficum 168

Cardamine 45 I

Carduus 508

Carlina 509

Carthamus 5 ro

Carum 23r

Caryophyllus $38 \mathrm{r}$

Cafia 2y9. 301

Cafline 239

Caftor 12

Ceanothus $1>6$

Centaurea $54 \mathrm{t}$

Ceratonia 632

Cervus $15-17$.

Charo byllum 225

Cheiranthus 456

Chelidonium 3?3

Chenopodium 189

Chryfanthemum

\section{2}

Cicer 485

Cichorium 505

Cicuta 221

Cirnicifuga 388

Cinchona 151

Cifiampelos 614

Ciltus 382

Citrus 491. 495

Clematis 306

Cobaltum 722

Coccus 47.48

Coshlearia $449 \cdot 450$

Cocos úgs
Coffea 153

Colchicum 270

Collinfonia 89

Columba 34

Coluber 39. 40

Conium 206

Convallaria $25 \%$

$25 \%$.

Convolvulus I46- Dianthus 3-6

$$
150
$$

Copaifera 322

Corallina 69

Cordia 172

Coriandrum 223

Coris 157

Corvus 27

Corylus 574

Coltus 73

Cratagus 35 I

Crocus 97

Cioton 58r-584

Curumis $591-593$

Cucurbita 589.590

Cuminum 220

Cuprefius 580

Cupram 720-722

Curcuma 75

Cufcuta 128-129

Cycus 635

Cyclane!! 39

Cynips 59. $5^{\text {I }}$

Cymomorium

559

Cynculatiom

134 Felis 7

Cyperus 103. 104
Cyprinus 44

Cytinus 557

L).

Daphne 278.279

Datura i6o

Daucus 205

Delphinium 384 .

Dencalium 65

Dietanunus 308

Diofpyros 630

Dolichos 480

Doronicum 530

Dorftenia 324

Dracoceplatura $43 \mathrm{x}$

Drofera 347

E.

Elephas 2

Epidendrum 55r.

Equifetum 634

Equus 2I

Ervum $483 \cdot 484$

Eryngium $200.20 \mathrm{I}$

Erylimum 454

1. fox 43

Eupatorium $\mathbf{5}^{\mathrm{I} \mathbf{2}}$

Euphorbia 3.36 .338

Euphratia 435

F.

Figara 122

Fenus 572

Eerrum 725-708 


\section{INDEX GENERVM.}

Ferula 200

Ficus 633

Formica 53

Fragaria 365

Fraxinus 6:8.629

Fumaria $470 .+71$

G.

Galega 488

Galium 116

Genifta 475.476

Gentiana $197-199$

Geranium 463

Germ 360.370

Glecoma 4 r?

Glyeyrrhiz 486

Gnaphalium 522

Gofypium á6's

Gratiola 83

Guajacum 30 ?

Guilandina 306

Gyprophila 324

Gypfum 675. 676

H.

Hiematoxylon $3 I$

Hedera $1 \div 9$

Hedyotis It4

Helix $6_{4}$

Helleborus 398.99

Helmintholirhus

$$
725 \cdot 726 \text {. }
$$

Heracleum 212

Herniaria 198

Hibilcus 469
Hieracium 504

Hippopotanus 22 Laferpition

Hirudo óo

Hirundo 35

Homo I

Hordeum 100

Huinus $75 \mathrm{I}$

Humulus 603

Hydrargyram 75

Hymenaea 298

Hyofryamus 16 I

Hypericum 497.498 Lichen $646-6_{53}$

Hyfopus tos

I.

Jafminum 78

Jitropha 585596

Isrnatia $17 \mathbf{1}$

J1ex 130

Jllicium 392

Imperatorid 226

Inula $527 \cdot 528$

Indigoters 487

Iris $98-102$

Iffs 67

Juglans 5 ? $\mathrm{x}$

Juniperus $615-6$ I3

K.

Kompferia 76

L.

Lacerta 38

M.

Iactuca 50I. 502 Madrepora 66

Lanium 4.18 Malva 4.67

$21 \mathrm{I}$.

Lavandula 4 To.4II

Laurus $286-292$

L.axr fonia 255

Leduri $3^{\mathrm{I}} \mathbf{5}$

Lcontodon 503

Leonurus $4 \geq 2$

l.epidiom $+45.4+6$

Lepus $1 \mathrm{I}$

Liguficum 213

Lilium 253

Linnsea $44 \pi$

linum 245.246

Liquidambar 575

Lithofpermum 3 [

Lobelia 546

Lonicera $15+\cdot 5_{5} 6$

Lucanus 45

Lumbricus 59

Lupinus 478

Lycoperdon $6_{56}$

657

Lycopodium 643 644

Lyfimachia $14 \mathrm{x}$

Lychrum 334 
Marania 74

Marmor 674

Marrubium $42 \mathrm{I}$

Matricaria 533.534

Melaienca 496

Melifía $429.43^{\circ}$

Meloe 46

Merifpermum 6 I 0

Mentha 4 I $2-4$ I 6

Menyanthes I 40

Mercurialis 609

Merpilus 353

Mica 682

Mimofa 625-627

Molybdznum 708

Momordica 588

Monatda 84

Monodon 24

Morus 566

Mofchus I4

Muria 69j-695

\section{Mus 13}

Myrica 597

Myriftica 560

Myroxylon 302

Myrtus $340.34^{2}$

Mytilus 63

N.

Natrum 688. 689

Nepeta 409

Nerium I 84

Nicotiana 162

Nigella 390

Nitrum $685 \cdot 687$ Phafeolus 479

Oryza 263

Oltrea 62

Oris 19

Oxalis $3 \geq 9$

P.

Pæonia 383

Panax 63I

Panicum IoZ

Parietaria 62I

Paris 285

Parnaffia 243

Pavo 3 I

Pensea I I 8

Perca 42

Petiveria 27I

Peziza 655

Phalena 49
Phafianus 32

Phoenix 659

Ochra 734.735 Phyllanthus 56I

Phyralis 165

Phyleter 26

Phytolacca 330

Pimpinella $=32.233$

Pinus $576-579$

Piper $90-92$

Piltacia $593-600$

Orchis $549.55^{\circ}$

Origanum $+23-4.6$ Pifum 481

Papaver 374.375

Paftinaca $22: .228$

Pedicularis 436

Peucedanum 208

Phalaris 106

Plantago I I 9. 120

Plumbago 145

Plumbum 715

Plumeria $185^{\circ}$

Polygala $4 \div 2$

Polygonum 290 . 283

Polypodium 640

Polytrichum 645

Populus 606. 607

Portulaca 333

Potentilla 366.367

Poterium 568

Primula 138

Prunella 434

Prunus $346-35^{\circ}$

Plychotria 152

Pterocarpus 473.

474

Pulmonaria I35

Pumex 732

Punica 343

Pyrices 7 IO-ZI 2

Pyrola 


\section{INDEX GENERVM.}

Pyrola 318-320 Sapindas 284 Pyrus 3540355 Q.

Qualfia 3r3. 3 I $_{4}$ Quercus 560

R.

Rana 36. $37^{\mathrm{T}}$

Ranunculus 397

Raphanus 462

Rhamnus 173-175

Rlyeum $29+295$

Rhodiola 608

Riododendrum316

Rhus $237 \cdot 238$

Ribes $177 \cdot 173$

Ricinus 587

Rola 358-36I

Rosmarinus 85

Rubia II7

Rubus $362-364$

Rumex 2ít-260)

Rufens 6I5. 616

Ruta 309

S.

Saccharum I05

Salicornia $7 ?$

Salix 595

Salvia 86-88

Sambucus240.241

Sanguiforba i2 I

Sanicula 202

Sintalum I 25

Santolina 5 r 3

Saponaria 325

Satureja 400.407
Saxifraga 323

Scabiofa I I 2. 113

Scandix $2=4$

Schitus 673

Scilia 254

Scorpio 55

Scorzonera 500

Scrophularia $\$ 39$

Sedum 3:7. 328

Semecarpus 230

Sempervivum 339

Sepia 6r

Sefamum 442

Sigesbeckia I 9 I

Silex 683. 684

Sinapis $46 x$

Silon 217

Sifymbrium $45^{2}$

Sium 216

Smilax 604. 605

Solanum I 6́6. I 67

Sulitiago 526

Sophora 296

Sorbus $35^{2}$

Spigelia 143

Spilanthus 5 I I

Spinacia $60 \mathrm{r}$

Spirvea $35^{6} .35 z$

Spnngia 68

Staribys 4:0

Stannum ?I 4

Stalice 244

Sribium 700
Stirium 677

Struthio 30

Strychnos 169.1 7o

Styrax 32 I

Succinum 706

Sus 23

Switenia 3I2

Symphytum I36 $T$.

Talcum 678-680

'Tamarindus 96

Tamarix 242

Tanacetum 5 I 4

Tartarus 730

Terminalia 623

Tetrao 33

Teucrium40I-405

Thea 380

Theobroma 493

Thlafpi $447 \cdot 448$

Thymis 427.428

Tilia 379

Toluifera 3 Io

Tophus 733

Tordylium 204

Tomentilla 368

Tragopogon +99

Trapa I23

Tribhechus 3

Trifolinm 400.40r

'Trigomella 492

Triticum 110. III

Tropurrolum 2-2

Turiilago 524

$\mathrm{X} 2$ 


\section{INDEX GENERVM.}

U.

Ulmus 196

Urfus 9. 10

Urtica 564.565

Uvaria 38 ?

$\mathrm{V}$.

Vaccinium 276.277

Valeriana 93-95

Veratrum 617.618 Vitis 180
Vitriolum 700.703

Viverra 8

WW.

Wintera 39 I

$\mathrm{X}$.

Xanthium 567

Z.

Zincum $719 \cdot 720$

\section{NDEX}

NOMINVM OFFICINALIVM.

$$
\text { I. ANIMALIA. }
$$

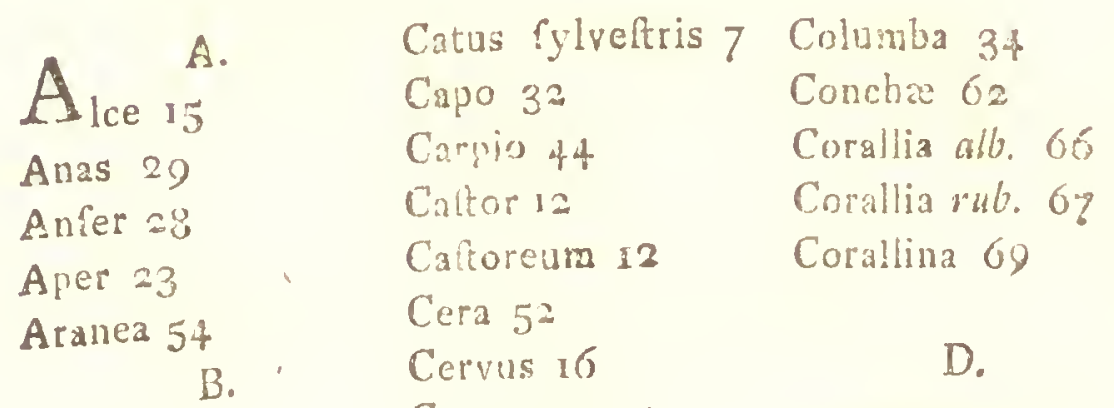

Bos 20

Bufo 37

Cetus $p r .25$

Crtus /p. 26

Dentalium 65

Chermes it?

Cancer 50.57

Corcionella 48

Canis of

Cochlox 64

Ebur 2

Cantharides 46

Colla pifciun at Equua 2 r 


\section{INDEX NOMINVM OFFICINALIVM.}

F.

Formica 5.3

Fungusrofarum so Ihis 33

G.

Gallac 5I

Galina 32

H.

Hidus is

Hippopotamus

Ifircus 18

Hirudo 60

Hirundo 35

Homo I

I.ac 20

\section{L.}

l.epus II

Lucius 43

Lumbricus 59

Lupus 5

M.

Nanati 3.

Ihel 52
Millepedes 58

Mofchus it

O.

Ovis 19

P.

Pavo 35

22 Perdix 33

Perlas 63

Perca 42

Pica 27

Pifcium colla $4 \mathrm{I}$

Porcus $=3$

Propolis 52

$\mathrm{R}$.

Rangifer 17

Ranæ 36

Rofarum fungus $5^{\circ}$

S.

Scarabrus 45
Scorpio 55

Sepia 6I

Sericum 49

Serpentes 40

Spongia 68

Stincus 38

Struthio 30

$\mathrm{T}$

Taxus io

Taurus 20

U.

Unicornu 24

Urfus 9

\section{V.}

Vipera 39

Vitulus 20

Vulpes 6

z.

Zibethum $\delta$

\section{I1. VEGETABILIA.}

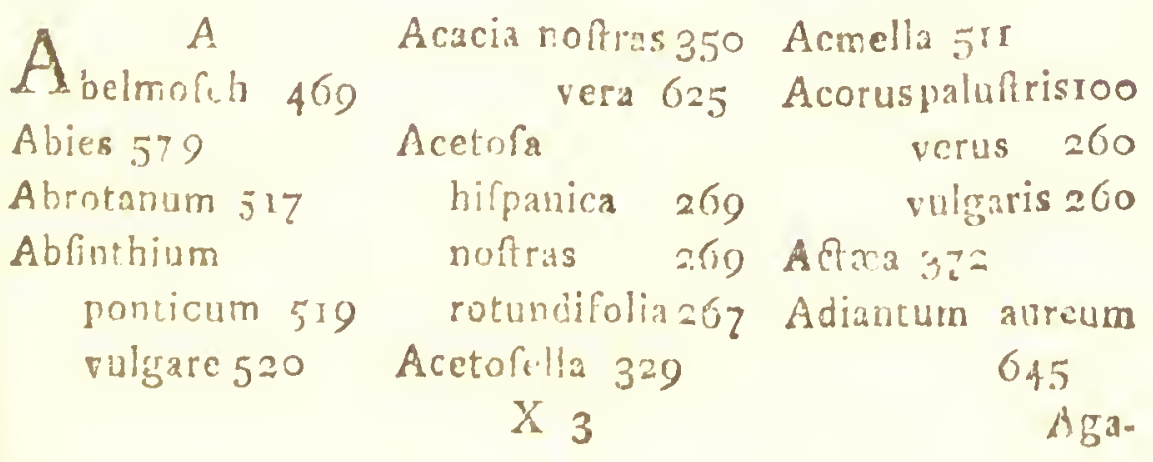




\section{INDEX NOMINVM OFFICINALIVM.}

Agaricus 654 Ageratum 538 Agnus cafus 443 Agrefta $\times 80$ Agrimonia 335 Alcanna vera 275 fpuria 133 Alchimilla $\quad 27$ Alkekengi 165 Allamanda I 82 Alliaria 455 Allium $25^{\mathrm{L}}$

Aloës lignum 670 fuccus 259 Altheca +65 Ammi verum 217 veterum I $\&$ I Ammoniacum 654 Amomum 342 Amygdala 345 Amyluin I Io

Anacardium

oricntale $=36$ occidentale 293 Anagiallis 142 Ananas $2+8$ Anugyris 207 Anethum 229 Angelica fativa2ra fylveftris 215 Anime 208

Anifutnvulgarez.33 freilatum 392 Anferina $3^{66}$ Anticholerica $=96$
Antirrhinum 438 Balfamum Anthophylli $38 \mathrm{r}$

Anthora 387

Anthos 85

Apium 235

Aquilegia 389

Arabicum gummi 625

Ariftolochia

fabacea $47^{\circ}$ longa 555 rotunda 5.54 trilob:ta 552 vulgaris $55^{6}$ Armoracia $45^{\circ}$ Arnica 529

Artemifia 518

Arum 558

Afarum 331

Afpalathum 67r

Aparagus $25^{6}$

Afphodelus 255

Alia fierida 209

Atriplex faíiva 6zz

rylvetris 190

Avena ros

Auratiun 495

Auricularia 114

Auricula Judar 655 muris $50 \div$ $B$.

Balauftia 343

Balfamum

Mecha $=7.4$
Copaiva 322

peruvianum 302

Balfamita $5^{\mathrm{I} 5}$

Barba hirci 499

Bardana 50?

Bafilicum 433

Bdellium 667

Beccabunga $8 \mathrm{r}$

Bedeguar 360

Behen album 542 oleum 306 rubrum 244

Belladonna 164 Bellis pratenfis 532 minor $53 \mathrm{r}$

Benzoë 623 Berberis 262 Beta rubra 194 Beta alba 195 Betonica 4igl Betula 562 Biftorta 280 Boletus 656 Bombax 468 BonusHenricus 189 Borrago 137 Botrys 191 Branca urfizi2.444 Braffica 459 Brafilia 303.304 Britannica 266 Bryunia 594 Butgloina 132

Burfa 


\section{INDEX NOMINVM OFFJCINALIVM.}

Burfa paftoris 448 Caflia caryoph.34r Cicuta 206. 22r.

Buxus 563

C.

Cacao 493

Cajeput +96

Calamintha +30

Calamus vulgaris 260

Cacitrapa $54+$

Calendula $5 \div 5$

Campefinanum 3 r

Camphorata 126

Camphora 3,38

Canarienfe $\int e m .106$

Canella alba $33^{2}$

Cannabis 602

Capraris $37^{\mathrm{r}}$

Caprifolium I $_{5}$

Capillusvener. $64^{2}$

Caranna $66 \%$

Cardiaca 422

Cardamine $4, \tau$

Cardamomum ?

Cardopatia 509

Carduus Marix .508 benedictus 543

Carica 633

Carlina 509

Carpobalfamum

274

Carthamus 5 ro

Caryophyllata 360 Caryophyllus 381

Carvi 231

Cafcarilla 58 I lignea -87 Cicutaria 235

Fittula 299 Cimicifnga 388

Caffine 130

Caltanea 572

Cataputia minor

337 Citrullus 590

major 537 Cocculi 610

Catechu 627

Ceanothus I 76

Centaurium minus

198

Centumuodia 282

Cepa 252

Cerafus rubr. 347 Colubrin. nigr. $\quad 348$

Cerefolium 224

Ceterach 637

Chamadrys 405

Chamamorus $36_{4}$ Confolida med.400

Chamxeitys 401

Chamomilla

noftras romana 535

Cheiri $45^{6}$

Chelidonium

majus

rivus 39 ?

Chenopodium anthelminthicum 392

China cortex $35 \mathrm{~L}$ radix 605

Cicer 485

Cichorium 505

X 4 regalis 384

Contrajerva 124

Conyza 528

Copriva 322

Copal 669

Coriandrum 223

Coris 157

Corylus 574

Coltus 73

Cotula foctida $53^{6}$ repitus lupi 657

Crocus 97

Cubcue 92

Cucumis $5 \% 3$

alininus 588

Cucur . 


\section{INDEX NOMINVM OFFICINALIVM.}

\begin{tabular}{|c|c|c|}
\hline $\mathrm{N}$. & Pareira brava 6 r 4 & lum 481 \\
\hline Japha 495 & Paradifi grana $\div 2$ & liftacia 598 \\
\hline Tapelius 386 & Parietaria 621 & Plantagro 119 \\
\hline apus 457 & Paris 285 & Plumeria 185 \\
\hline ardus 620 & Pafiulac 179 & Polium 402 \\
\hline afturtium & laca 227 & Polypodium 640 \\
\hline aquaticum 452 & 1 a 58.3 & Poma Lorftorphia- \\
\hline hortenfe 445 & in 292 & na 35 t \\
\hline indicum 272 & Pedicularis 436 & Populus 606 \\
\hline Nepeta 409 & Pentaphyllum 367 & Porrun 249 \\
\hline Nephriticum 306 & Peragua 239 & Portulaca 333 \\
\hline Nige!la 390 & Perfoliata 203 & Primula veris 138 \\
\hline Ninfi 216 & Perfica 344 & vium $18+$ \\
\hline Norlandica bacca & Perlicaria $28 \mathrm{r}$ & ola $3+9$ \\
\hline $3^{6} 3$ & rumbalf.302 & $11 \mathrm{a} 434$ \\
\hline Nummularia $14 \mathrm{r}$ & Petalites $5=5$ & us 349 \\
\hline Nux aquatica 123 & Petrofelinum & Pfyllium 120 \\
\hline $\begin{array}{c}\text { Nympliaca } 377 \\
0 .\end{array}$ & $\begin{array}{l}\text { nottras } 234 \\
\text { macedonicum } 218\end{array}$ & $\begin{array}{l}\text { Ptarmica } 539 \\
\text { Pulegium } 416\end{array}$ \\
\hline Oliva 79 & Peucedanum 208 & Pulmonaria \\
\hline Dlibanum 613 & Phafeolus 479 & ulc fa 135 \\
\hline Onoris 477 & Phu 94 & arborea 648 \\
\hline Opium 375 & Phytolacca 3.30 & Pulfatilla 394 \\
\hline Орорапах 228 & Pichurim 20: & Pyrethrum 537 \\
\hline Driganum & Pimenta 342 & Pyrola $318-320$ \\
\hline vulgare 425 & $\begin{array}{l}\text { Pompratia } \\
\text { italica }\end{array}$ & Quaffia \\
\hline rleana 378 & italica 568 & Cuercus $57^{\circ}$ \\
\hline robus $48 t$ & noftras 232 & \\
\hline $\begin{array}{c}\text { ryza } 263 \\
\text { P. }\end{array}$ & $\begin{array}{c}\text { Pinus fativas } 60.577 \\
\text { fylveftris } 576\end{array}$ & Ranunculus albus \\
\hline ia 3 & Piper rotundum 90 & Rapa 458 \\
\hline mac 658 & longum $9 \mathbf{I}$ & Raphanus 462 \\
\hline aver? & indicum 168 & Refina elaftica \\
\hline
\end{tabular}




\section{INDEX NONINVM OFFICINALIVM.}

Rhabarbverum 295 Salix 595

monachorum $=68$ Salvia 86

Rhaponticum 294 Sambucus $24 \mathrm{c}$

Rloodia 608

Rhodium 475

Rhodod. Chry-

fanth. 316

Rhœes 374

Ribes nigrum 178

rubrum 177

Ricinus vulgaris

587

major 533

Roccella $65^{2}$

Rofa rubra 358

damafcena 359

fylveftris 360

alba $36 r$

Rofmarinus

hortenfis 85

fylveftris $3^{T 5}$

Ros folis $2+7$

Rubia II?

Rubus idæus 362

Rufcus 6r5

Ruta hortenfis 309

muraria 639

S.

Sabadilla 6I8

Sabina 6rI

Saocharum 105

Sagrapenum 668

Sagu 6.35

Salep $55^{\circ}$

Salicaria 334
Sandaraca 612

Sanguis draconis 469

Sanicula 202

Santalum 474

Sanctum lignum

\section{7}

Santolina 5r3

Santonicum 5 56

Saponaria 325

Saponaria 284

Sapotilla $26 r$

Sapan 305

Sarcocolla 118

Sarfaparilla 604

Saflifras $29 r$

Satur.ja 406

Satyrium 549

Saxifraga slba 323 rubra $35^{6}$

Scabiofa iz

Scammonicm $1 \neq 6$ Sorbus

Sclarea 38

Schoenanthus 6 ro

Scolopendrium6,6

Scorodonia 27 r

Scrophularia

aquatica 440

factida 439

Scordium 404

Scorzonera 500

Sebeftena 172
Sedum minus $\mathbf{3 2 8}$

Sempervivum 339

Senna 300

Senega 472

Senegal gum б 26

Serpentum rad. +4

Serpentinum lignum 624

Serpentaria virgi-

nica 553

Serpyllum 427

Sefamum 442

Sereli cretic. 204

Sigillum Salomo. nis 258

Silermontanum $\approx$ ir

Simaruba 314

Siliqua dulcis 623

Sinapi $46 \mathrm{r}$

Soda 77

Soja 480

Solanaar 167

Soldanclla $5^{\circ}$

Sophia 453

domeftica $35 \mathrm{r}$

aucuparia $35^{2}$

Spatula foctida ror

Spica 410

indica 620 celtica 95

Spigelia 143

Spinacia cor

Spina cervina ry3

Squilla 


\section{INDEX NOMINVM OFEICINALIVM.}

Squilla 254

Staphifagria 385

Stcechasarabicatir

citrina 522

Storax calamitaz=r liquida 575

Stramovium

Struthium

Suber 569

Silmach 237

Symphoricarpus

\section{$1 \div 5$}

T.

Tabacum I62

Tacamshaca 607 Tunica 326

Tamarindus o6

Tamarifus 242

Tanacetum 5 it

Taraxacum 503

Telephium 327

Terebinthina

communis 5.6

cypria $50 ?$

venet:

578

Terra japonica 62? Tharpi 147

Thea 380

Thymus vulgaris

Tigiia 583

'lilia 3\%?

Tolu 306

Trifolium

album

$\mathrm{V}$.

Vanilla $55^{\mathrm{I}}$

Verbena 83

Vernix 233

Veronica 80
Thus 6r3

Thymiama 573

\section{creticus}

Tormentilia 368

Tornefol 575

Tragacantha 489

Trichonanes 638

$49 \mathrm{I}$

aquaticam 1 \& 0

Turpethum I 4 ?

Tufilago 524

Valeriana minoro3

major 94

Verbafcum 558.159

ViRcoriális 2.50

Vinca perviuca Is3 Lingiber $7^{\circ}$
Vinum r8o

Viola 547

Violaria 543

Virga aurea 526

Vircum 596

$V$ itis 180

idara 277

Uimus 396

Ulmaria 357

Vomica 369

Urtica major 545

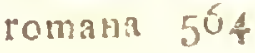

U) fnea 646

Uva urfi 35

Uvularia or

Vulvaria 193

II.

Winteranus $39 \mathbf{r}$

$\mathrm{X}$.

$X_{\text {anthium }} 5^{67}$

Xylobalfamum 274

$X$ yris Ior

\section{Z.}

Tedonria 76 


\section{INDEX NOWHVM OFFICINALIVM.}

\section{III. $L A P I D E S$.}

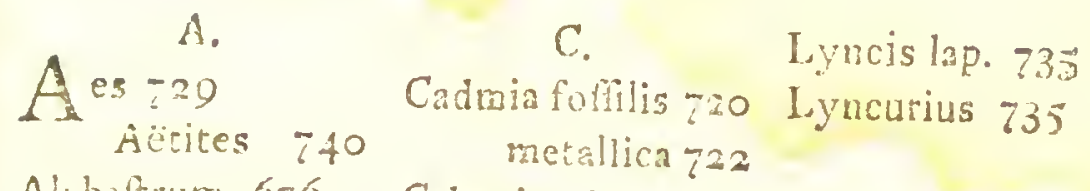

Alabaftum 6-6

Alumen crudum

Colaminaris 720

M.

Calx viva $0_{7} 4$

607 Carabe 706

plumofum 68 I Carneolus 684

rochie 698 Chalybs 725

feiffle $67 \%$ Cinnabaris 76

Ainbra $\% 04$

Cabaltum 723

Amethyftus 687 Colonients terra

Ammoniacum 606

Anglicana terra 75

Antimonium 718

Aquilis lap. 740

A:gentum 732 748

Creta 749

Cryftallus 680

Argentum vivum

\section{3}

Armenus lap. $73 \mathrm{I}$

Arfenicum 7.3 .714

Afphaltum 809

Auripigmentum

7 I 2

Aurum 733

B.

Bezoar 736

Bifmutum 72 I

Bitumen jud. 809 Bolus $7+7$

Borax 690
G.

Glacies Marice 689

Giranatus 6ya

Gypium 675

H.

Haematites 727

Hyacinthns 687

I.

Judaicus 734

Lac lunae 750

lazuli lap. 730

Lemnia terra 745 Fubinus 609 Lunac lac 750
Magnes 728

Malaccenfis 737

Marcafica 725

Margaritie 738

Piercurius $7 \mathrm{Ig}$

Muruia 708

N.

Nepbriticus 680

Nigrica 673

Nicrua 685

o.

Ochra 643

Oleum petrx 707

Olteoculla 74 I

P.

Petroleum $70 ?$

Plumbago 717

Plumbum $7 \approx 4$

Porcinus 737

Punex 741

R.

Rubrica 178 


\section{INDEX NOMINVM OFFICINALIVM:}

\section{S. Smiris $726 \quad$ Tripolitana terra}

Saccharum Saturni 703

Sal ammoniacum

$$
696
$$

anglicum 688

commune 693

$$
694
$$

gemmæ 695 Talcumalbum 682

Sapphirus 699

Sigillata terra 747

Silex 683

Smaragdus 69r
Specularis 689

Stannim 723

Stenomarga $75^{\circ}$

Succinum 706

Sulphur 710. "? II

$\mathrm{T}$.

viride 679

Tartarus 730

Terra figill. 747

Topafius 69I
746

\section{V.}

Viride mont. 744

Vitriolum

album $\quad 702$ cyprium $70 \mathrm{~K}$ martis 700

Umbra 748

\section{Z.}

Zincum 719

\section{F I N I S.}

Pag. 159. lin. 19. 1. ACTAEA racemofa

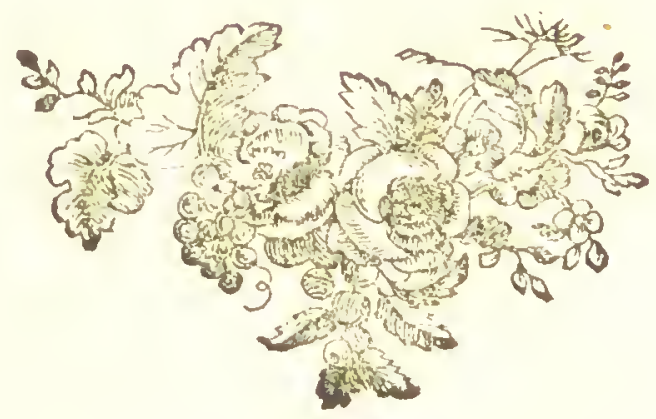



